\title{
Monte Carlo Simulations of Temperature-Programmed and
} Isothermal Desorption from Single-Crystal Surfaces

\author{
Dissertation \\ of \\ Stephen J. Lombardo \\ August 13, 1990 \\ Department of Chemical Engineeering \\ University of California, Berkeley \\ and \\ Material and Chemical Sciences Division \\ Lawrence Berkeley Laboratory \\ University of California \\ Berkeley, CA 94720
}

This work was supported by the Director, Office of Basic Energy Sciences, Chemcial Sciences Division of the US Department of Energy under contract DE-AC03-76SF00098.

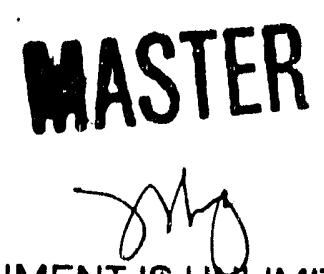


Monte Carlo Simulations of Temperature-Programmed and Isothermal Desorption from Single-Crystal Surfaces

\title{
by
}

Stephen J. Lombardo

\begin{abstract}
The kinetics of temperature-programmed and isothermal desorption have been simulated with a Monte Carlo model. Included in the model are the elementary steps of adsorption, surface diffusion, and desorption. Interactions between adsorbates and the metal as well as interactions between the adsorbates are taken into account with the Bond-Order-Conservation-Morse-Potential mathod.

The shape, number, and location of the TPD peaks predicted by the simulations is shown to be sensitive to the binding energy, coverage, and coordination of the adsorbates. In addition, the occurrence of lateral interactions between adsorbates is seen to strongly effect the distribution of adsorbates on the surface.

Temperature-programmed desorption spectra of a single type of adsorbate have been simulated for the following adsorbate-metal systems: $\mathrm{CO}$ on $\mathrm{Pd}(100) ; \mathrm{H}_{2}$ on $\mathrm{Mo}(100)$; and $\mathrm{H}_{2}$ on $\mathrm{Ni}(111)$. The model predictions are in good agreement with experimental observation. TPD spectra have also been simulated for two species coadsorbed on a surface; the model predictions are in qualitative
\end{abstract}


2

agreement with the experimental results for $\mathrm{H}_{2}$ coadsorbed with strongly bound atomic species on $\mathrm{Mo}(100)$ and $\mathrm{Fe}(100)$ surfaces as well as for $\mathrm{CO}$ and $\mathrm{H}_{2}$ coadsorbed on $\mathrm{Ni}(100)$ and $\mathrm{Rh}(100)$ surfaces. Finally, the desorption kinetics of $\mathrm{CO}$ from $\mathrm{Pd}(100)$ and $\mathrm{Ni}(100)$ in the presence of gas-phase $\mathrm{CO}$ have been examined. The effect of pressure is seen to lead to an increase in the rate of desorption relative to the rate observed in the absence of gas-phase $\mathrm{CO}$. This increase arises as a consequence of higher coverages and therefore stronger lateral interactions between the adsorbed 00 molecules.

Geniritell 
To

Mariette,

Michael,

Mom,

and Dad 
Table of Contents

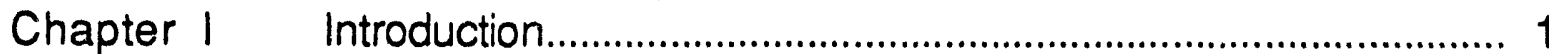

Chapter II A Review of Theoretical Models of Adsorption, Diffusion, Desorption, and Reaction of Gases on Metal Surfaces

Chapter III A Monte Carlo Model for the Simulation of Temperature-Programmed Desorption Spectra...........158

Chapter IV Monte Carlo Simulation of the TemperatureProgrammed Desorption of Coadsorbed Species......... 201

Chapter V Monte Carlo Simulation of the Effect of Pressure on Isothermai and Temperature-Programmed Desorption Kinetics.

Appendix FORTRAN Programs for Simulating TemperatureProgrammed and Isothermal Desorption. 
List of Figures

Chapter II

Fig. 2.1 a. Potential energy surface for molecular adsorption as a function of orientation angle $\theta$ and distance from the surface $z$. The energy contours are in eV. b. One-dimensional potential versus reaction coordinate s [36].

Fig. 2.2 a. Potential energy surface for dissociative adsorption as a function of distance from the surface $z$ and internal bond distance $r$. The energy contours are in eV. b. One-dimensional potential versus reaction coordinate $s[36]$.

Fig. 2.3 Variation of $S(1)(\theta) / S(1)(0)$ with $\theta$ for adsorption via a precursor mechanism. The precursor parameter $K=k_{a}^{*} / k^{*}$.

Fig. 2.4 a. Variation in $f_{d}^{\prime}$ with $\theta$ at $T=300 \mathrm{~K}$ [26]. The nomenclature $(5,6)$ corresponds to $W A A=5 \mathrm{~kJ} / \mathrm{mol}$ and $z=6$, respectively. b. Variation in $f_{d} "$ with $\theta$ [24]. The nomenclature $(2,6)$ corresponds to $\mathrm{WAA}_{\mathrm{A}}=2 \mathrm{~kb} T$ and $z=6$, respectively.

Fig. 2.5 Variation of $v_{d}(1)(\theta) / v_{d}(1)(0)$ and $\Delta E_{d}(\theta)$ with $\theta$ for repulsive (top panels) and attractive (bottom pancls) interactions [26]. Solid line: $|W A A|=5 \mathrm{~kJ} / \mathrm{mol}, z=6$; dashed line: $\left|W_{A A}\right|=5 \mathrm{~kJ} / \mathrm{mol}, z=4$; dotted line: $\left|W_{A A}\right|=$ $3 \mathrm{~kJ} / \mathrm{mol}, \mathrm{z}=6$.

Fig. 2.6 Variation in $f_{r}$ with $\theta$ [25]. The nomenclature $(1,0,3)$ corresponds to $W_{A A}=k_{b} T, W_{A B}=0$, and $W_{B B}=3 k_{b} T$, respectively.

Fig. 3.1 Simulated $(0)$ and experimental $(\mathbf{A})$ variation of $S(1)(0)$ versus $E_{T} \cos \theta_{i}$ for $X_{e}$ adsorption on $\mathrm{Pt}(111)$ [46]. $E_{T}$ is the incident kinetic energy and $\theta_{i}$ is the incident 
angle measured from the surface normal

Fig. 3.2 Simulated $(0)$ variation of $S(2)(0)$ versus $E_{T} \cos ^{2} \theta_{i}$ for $\mathrm{H}_{2}$ adsorption on $\mathrm{Cu}(100)$ [31]. $E_{T}$ is the incident kinetic energy and $\theta_{i}$ is the incident angle measured from the surface normal. The solid line is a guide to the eye.

Fig. 3.3 Simulated (solid line) [53] and experimental (circles) [54] variation of $S(2)(0)$ versus incident kinetic energy for $\mathrm{N}_{2}$ adsorption on $\mathrm{W}(110)$.

Fig. 3.4 a. Potential of mean force versus height above the surface for $\mathrm{CO}$ on $\mathrm{Ni}(111)$. b. Simulated variation of $\mathrm{S}(1)(0)$ versus $\mathrm{T}$ for $\mathrm{CO}$ adsorption on $\mathrm{Ni}(111)$ [36].

Fig. 3.5 Simulated variation of $S(2)(0)$ versus incident kinetic energy for $\mathrm{H}_{2}$ adsorption on $\mathrm{Ni}(100)$ [42]

Fig. 3.6 Variation in $S(2)(\theta) / S(2)(0)$ versus $\theta$ for repulsive nearest-neighbor interactions [59].....

Fig. 3.7 Variation in $S(1)(\theta) / S(1)(0)$ versus $\theta$ when nearestneighbor interactions occur [60]

Fig. 3.8 a. Variation in $S(2)(\theta) / S(2)(0)$ versus $\theta$ when precursor states exist for a fixed value of $\eta$. b. Variation in $S(2)(\theta) / S(2)(0)$ versus $\theta$ when precursor states exist for a fixed value of $L$ [59].

Fig. 3.9 Experimental and predicted variation in $S(2)(\theta)$ versus coverage for $\mathrm{N}_{2}$ adsorption on $\mathrm{W}(100)$ [59]

Fig. 3.10 Experimental (error bars) [62,63] and predicted [61] variation in $S(1)(\theta)$ versus coverage for $N_{2}$ adsorption on Ru(001).

Fig. 4.1 Experimental [69] and predicted [79] variation in D versus $1 / \mathrm{T}$ for $\mathrm{Rh}$ diffusion on $\mathrm{Rh}(100)$. 
Fig. 4.2 Variation in $D$ with $1 / T$ for hydrogen diffusion on $W(110)$. Symbols are the experimental data $[90,91]$ and the lines are the model predictions [89]....

Fig. 4.3 a. Predicted variation in $D$ with $\theta$ for hydrogen diffusion on a metal surface at $27 \mathrm{~K}$ [92]. $b$. Experimental variation in $D$ with $\theta$ for hydrogen diffusion on $W_{(110)}$ at $27 \mathrm{~K}[91]$.

Fig. 4.4 Variation in $D(\theta) / D(0)$ with $\theta$ for repulsive interactions for $W_{A A}=k_{b} T^{*}$, where $T^{*}$ is the standard temperature [93].

Fig. 4.5 Variation in $D(\theta) / D(0)$ with $\theta$ for nearest- $\left(w_{1}\right)$ and next-nearest-( $\left.w_{2}\right)$ neighbor interactions [94]....

Fig. 4.6 Variation in D with $W_{A A} / k_{b} T$ [98]. The open circles are from simulations using eq. 4.3 and the closed circles are from simulations using eq. 4.5. The crosses are the rescaled diffusivities from eq. 4.5 .

Fig. 4.7 Variation of $D$ with $\theta$ for repulsive ( $\mathbf{D})$, attractive ( $\Delta$ ), and no nearest-neighbor interactions $(\bullet)$ [99]...................

Fig. 4.8 a. Simulation of the $\theta$ dependence of $D$ for repulsive nearest-neighbor and attractive next-nearest neighbor interactions [100]. b. Experimental variation in $D$ with $\theta$ for oxygen on $W(110)[101]$

Fig. 4.9 Variation in $D(\theta) / D(0)$ with $\theta$ for equivalent nearest$\left(w_{1}\right)$ and next-nearest-( $\left.w_{2}\right)$ neighbor repulsive interactions [104]. a. Disordered region. b. Ordered region.

Fig. 4.10 a. Variation in $D\left(\theta_{A}\right) / D(0)$ with $\theta_{A}$ in the presence of an immobile adsorbate $B$ for $\theta_{B}=0(\square), \theta_{B}=0.15(O)$, and $\theta_{B}=0.36(\triangle)$. b. Variation in $D\left(\theta_{A}\right) / D(0)$ with $\theta_{B}$. 
The filled symbols represent upper limits [105]

Fig. 5.1 Arrhenius plot for $\mathrm{Ar}$ and $\mathrm{Xe}$ desorption from $\mathrm{Pt}(111)$.

Solid and dashed curves are dynamically corrected and uncorrected TST, respectively [29].

Fig. 5.2 Activation energy (a) and preexponential factor (b) profiles versus $\mathrm{T}$ for $\mathrm{CO}$ desorption from $\mathrm{Ni}(111)$ [36]....107

Fig. 5.3 a. Predicted CO TPD spectra from a $W$ ribbbon [124]. b. Experimental CO TPD spectra from a $W$ ribbbon [125]. The $\mathrm{CO}$ exposures ( $\mathrm{Pa} \mathrm{s}$ ) are: a) $1.9 \times 10-5$; b) $6.0 \times 10-5$;

c) $1.6 \times 10.4$; d) $1.9 \times 10.4$; e) $2.5 \times 10.4$; and f) $3.1 \times 10-3$.

c. Predicted activation energy profile versus coverage [124]. 108

Fig. 5.4 Predicted [120] and experimental [127] CO TPD spectra from $\operatorname{lr}(110)$ for $\theta \mathrm{co}^{\circ}=0.82$ 109

Fig. 5.5 Predicted [126] and experimental [128] $\mathrm{O}_{2}$ TPD spectra from $\operatorname{Ir}(110)$. Curves in both panels correspond to oxygen coverages of $1.0,0.75,0.50$, and 0.25 .

Fig. 5.6 Variation in $\Delta \mathrm{E}_{d}(\theta)$ and $v_{d}(1)(\theta) / v_{d}(1)(0)$ versus coverage when surface reconstruction occurs [60]

Fig. 5.7 Comparison of Monte Carlo and continuum models for the simulation of non-associative TPD spectra in the absence of lateral interactions [132]. 112

Fig. 5.8 a. Monte Carlo simulation of TPD spectra [133]. b. Continuum simulation of TPD spectra [134]. Lateral interactions between adsorbates in the continuum simulations are described with the Braggs-Willaims approximation

Fig. 5.9 Simulation [135] of TPD spectra for both nearestneighbor interactions $w_{1}$ and next-nearest neighbor interactions w2. a. Continuum model. b. Monte Carlo 
model. The lateral interactions are in $\mathrm{kcal} / \mathrm{mol} \ldots \ldots \ldots \ldots \ldots . . . . .114$

Fig. 5.10 Experimental (a) $[62,63]$ and simulated (b) [61] TPD spectra of molecular $\mathrm{N}_{2}$ from $\mathrm{Ru}(001)$.

Fig. 5.11 Simulated (a) [132] and experimental (b) [136] TPD spectra of $\mathrm{H}_{2}$ from $\mathrm{Mo}(100)$.

Fig. 5.12 Simulated (a) [132] and experimental (b) [137] variation in $E_{d}$ with $\theta_{H}$ for $\mathrm{H}_{2}$ desorption from $\mathrm{Mo}(100)$.

Fig. 5.13 a. Simulated TPD spectra of $B$ and $A_{2}$ [139]. $b$.

Experimental TPD spectra of $\mathrm{CO}$ and $\mathrm{H}_{2}$ [142]. The dashed curves correspond to desorption of each species adsorbed separately, and the solid curves correspond to desorption of both species coadsorbed.

Fig. 5.14 Variation in $E_{d}$ with $\theta_{i}$ ior the simulations in fig. 5.13a [139]. The dashed curves; correspond to desorption of each species adsorbed separately, and the solid curves correspond to desorption of both species coadsorbed.......119

Fig. 6.1 Theoretical [126] and experimental [160] TPSR spectra for $\mathrm{CO}$ oxidation and $\mathrm{CO}$ desorption on $\operatorname{lr}(110)$.

Fig. 6.2 a. Predicted variation of $E_{d}$ versus $\theta$ for $C O$ desorption from $\operatorname{lr}(110)$ [126]. b. Predicted variation of $E_{r}$ versus $\theta$ for $\mathrm{CO}$ oxidation [126]......

Fig. 6.3 TPSR spectra for CO oxidation on $\operatorname{Ir}(111)$. a. Experimental spectra as a function of gas exposure [163]. b. Theoretical spectra as a function of coverage [161]

Fig. 6.4 Steady-state rate of $\mathrm{CO}$ oxidation versus $\mathrm{T}$ on Ir(111). Solid lines are theoretical calculations [162]; circles are experimental data in arbitrary units [163]; and dashed line is experimental data [164].

a. $P_{C O}=P_{O}=1.1 \times 10.7$ Torr. b. $P_{C O}=5 \times 10-8$ Torr and 
$\mathrm{PO}=1.2 \times 10-7$ Torr

Fig. 6.5 Steady-state rate of hydrogen oxidation versus $T$ on $\mathrm{Pt}(111)$ for $\mathrm{PH}_{\mathrm{H}}=1.34 \times 10.5 \mathrm{~Pa}$ and $\mathrm{PO}=6.7 \times 10-6 \mathrm{~Pa}$. Solid line is the theoretical calculation [165] and the dashed line is the experimental result [166]....

Fig. 6.6 Rate of $\mathrm{CO}_{2}$ production versus $\mathrm{t}$ for the reaction of $\mathrm{CO}$ with preadsorbed oxygen for $\mathrm{PCO}=1.0 \times 10.8$ Torr and $\theta 0 \approx 2 / 3$ monolayer [175]. Solid lines are the experimental results and the dashed lines are the model predictions

Fig. 6.7 Concentration profiles versus $t$ for $\mathrm{CO}$ oxidation for. an inlet step change from $2 \% \mathrm{CO}$ to a $1 \% \mathrm{CO}$ and $3 \% \mathrm{O}_{2}$ mixture [183]. Circles are the experimental data and the solid line is the model preciction.

\section{Chapter III}

Fig. 1 Comparison of Monte Carlo and continuum models for the simulation of first order desorption where $E_{d}$ is constant. (See Table 1 for parameter values).

Fig. 2 Comparison of Monte Carlo and continuum models for the simulation of second order desorption where $E_{d}$ is constant. (See Table 1 for parameter values)

Fig. 3 Illustrations of the effects of $M-A$ and $M-A$ plus $A-A$ interactions on the TPD spectra for second order desorption. (See Table 1 for parameter values).

Fig. 4 Illustrations of the effects of $M-A$ and $M-A$ plus $A-A$ interactions on the average $E_{d}$ for second order desorption.

Fig. 5 Illustrations of the effects of $M-A$ and $M-A$ plus $A-A$ interactions on $f(C N+1)$ for second order desorption....... 178 
Fig. 6 Monte Carlo simulations of $\mathrm{CO}$ desorption from Pd(100). (See Table 1 for parameter values).... 181

Fig. 7 TPD spectra for CO desorption from $\mathrm{Pd}(100)$ [23]............182

Fig. 8 Variation of tha average $E_{d}$ with $\theta c o$ for $C O$ desorption from $\mathrm{Pd}(100)$

Fig. 9 TPD spectra for $\mathrm{H}_{2}$ desorption from $\mathrm{Mo}(100)$ [26]

Fig. 10 Monte Carlo simulations of $\mathrm{H}_{2}$ desorption from $\mathrm{Mo}(100)$. (See Table 1 for parameter values).

Fig. 11 Variation of the average $E_{d}$ with $\theta_{H}$ for $\mathrm{H}_{2}$ desorption from Mo(100).

Fig. 12 TPD spectra for $\mathrm{H}_{2}$ desorption from $\mathrm{Ni}(111)$. Panel a is from ref. [37] and panel $b$ is from ref. [36].

Fig. 13 Monte Carlo simulations of $\mathrm{H}_{2}$ desorption from $\mathrm{Ni}(111)$. (See Table 1 for parameter values)

Fig. 14 Variation of the average $E_{d}$ with $\theta_{H}$ for $\mathrm{H}_{2}$ desorption from $\mathrm{Ni}(111)$

\section{Chapter IV}

Fig. 1 a. Simulation of $A_{2}$ desorption. See table 1 for model parameters. b. Variation of the average $E_{d}$ with $\theta A \ldots \ldots \ldots . .215$

Fig. 2 Surface map for $A(\bullet)$ at $\theta A=1.0$ for the simulation in fig. 1a. The open circles are metal atoms.

Fig. 3 a. Simulation of $A_{2}$ desorption with $\theta_{B}=0.25$. Curve 1 is for $Q_{B, n}=140 \mathrm{kcal} / \mathrm{mol}$ and curve 2 is for $Q_{B, n}=190$ $\mathrm{kcal} / \mathrm{mol}$. See table 1 for model parameters. $b$. Variation of the average $E_{d}$ with $\theta_{A}$

Fig. 4 Surface map for $A(\bullet)$ and $B(\square)$ at $\theta_{A}=1.0$ and $\theta B=0.25$ 
for the simulations in fig. 3a. The open circles are metal atoms.

Fig. 5 a. Simulation of $A_{2}$ desorption with $\theta_{B}=0.50$. Curve 1 is for $Q_{B, n}=140 \mathrm{kcal} / \mathrm{mol}$ and curve 2 is for $Q_{B, n}=190$ $\mathrm{kcal} / \mathrm{mol}$. See table 1 for model parameters. b. Variation of the average $E_{d}$ with $\theta_{A}$.

Fig. 6 a. Simulation of $A_{2}$ desorption with $\theta B=0.25$ when attractive $A-B$ interactions occur. Curve 1 is for

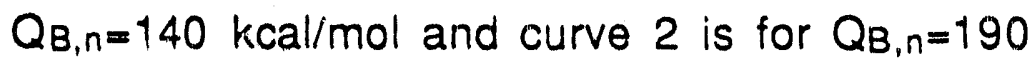
$\mathrm{kcal} / \mathrm{mol}$. See table 1 for model parameters. $b$. Variation of the average $E_{d}$ with $\theta_{A}$

Fig. 7 a. Simulation of $A_{2}$ desorption with $\theta_{B}=0.50$ when attractive $A-B$ interactions occur. Curve 1 is for $Q_{B, n}=140 \mathrm{kcal} / \mathrm{mol}$ and curve 2 is for $Q_{B, n}=190$ $\mathrm{kcal} / \mathrm{mol}$. See table 1 for model parameters. b. Variation of the average $E_{d}$ with $\theta_{A}$

Fig. 8 Surface map for $A(\bullet)$ and $B(-)$ at $\theta_{A}=1.0$ and $\theta_{B}=0.25$ for the simulation in fig. $6 a$. The open circles are metal atoms.

Fig. 9 a. Simulation of pure component desorption for $A_{2}$ and B. Curve 1 is for $Q_{A, n}=55 \mathrm{kcal} / \mathrm{mol}$ and curve 2 is for $Q_{A, n}=52 \mathrm{kcal} / \mathrm{mol}$. Curve 3 is for bridge-bonded species $B$ with $Q_{B, n}=33 \mathrm{kcal} / \mathrm{mol}$. Curve 4 is for on-top bonded species $B$ with $Q_{B, n}=33 \mathrm{kcal} / \mathrm{mol}$. See table 2 for model parameters. b. Variation of the average $E_{d}$ with coverage.

Fig. 10 a. Simulation of $A_{2}$ and $B$ codesorption for case I. See table 2 for model parameters. b. Variation of the average Ed with coverage.

Fig. 11 Surface map for $A(\square)$ and $B(0)$ at $\theta_{A}=0.51$ and $\theta_{B}=0.32$ for the simulation in fig. 10a. Open circles are metal atoms. 
Fig. 12 a. Simulation of $A_{2}$ and $B$ codesorption for case II. See table 2 for model parameters. b. Variation of the average $E_{d}$ with coverage.

Fig. 13 a. Simulation of $A_{2}$ and $B$ codesorption for cass III. See table 2 for model parameters. b. Variation of the average $E_{d}$ with coverage.

Fig. 14 Surface map for $A(-)$ and $B(-)$ at $\theta_{A}=0.52$ and $\theta_{B}=0.50$ for the simulation in fig. 13a. The open circles are metal atoms

Fig. 15 Experimentally observed TPD spectra for $\mathrm{H}_{2}$ and $\mathrm{CO}$ desorption from $\mathrm{Ni}(100)$ [18]. The dashed liriss are for desorption of each species adsorbed separately, and the solid lines are for desorption of both species coadsorbed.

\section{Chapter V}

Fig. 1 Variation in CO coverage versus time on polycrystalline $\mathrm{Pd}$ at $\mathrm{T}_{\mathrm{s}}=380 \mathrm{~K}$ (taken from Yamada et al. [6]). The open circles represent $9 T$ and the filled symbols represent the decay of $\theta\left(C^{18 O}\right)$ for $t>t_{1}$. a) The isotope switch was made at a) $t_{1}=20 \mathrm{~s}(\mathbf{0}), 40 \mathrm{~s}(\mathbf{\nabla}), 60 \mathrm{~s}(\mathbf{)})$, and $120 \mathrm{~s}(\boldsymbol{\nabla})$; b) at $t_{1}=10 \mathrm{~s}(\bullet), 20 \mathrm{~s}(\mathbf{\square}), 40 \mathrm{~s}(\bullet), 60 \mathrm{~s}(\boldsymbol{\nabla})$, and $120 \mathrm{~s}(\mathbf{\Delta}) ; c)$ at $t_{1}=5 \mathrm{~s}(\bullet), 10 \mathrm{~s}(\mathbf{\Delta}), 20 \mathrm{~s}(\bullet)$, and $60 \mathrm{~s}(\nabla) ;$ d) at $\mathrm{t}_{1}=5 \mathrm{~s}(\mathbf{v}), 10 \mathrm{~s}(\mathbf{v}), 20 \mathrm{~s}(\bullet)$, and $60 \mathrm{~s}(\bar{\eta})$ 255

Fig. 2 Variation of $k_{d} P$ versus $P$. The points designated by (C) are from the analysis of experimental data presented by Y'amada et al. [5] and the points designated by ( $\mathbf{m}$ ) are the values obtained from the Monte C.arlo simulations. 


\section{$x$ ii}

Fig. 3 Simulated variation in CO coverage versus time on $\mathrm{Pd}(100)$ at $\mathrm{T}_{\mathrm{s}}=380 \mathrm{~K}$. The open circles represent $\theta T$ and the filled symbols represent the decay of $\theta(C 180)$ for $t>t_{1}$. a) The isotope switch was made at $\theta(C 180)=0.30(-)$ and $0.60(\$)$. b-d) The isotope switch was made at $\theta\left(C_{180}\right)=0.30(\bullet), 0.60(\downarrow)$, and $0.85(\nabla)$

Fig. 4. Variation in the average value of $E_{d}$ as a function of $\theta \mathrm{T}$. Curve $a$ is for the $\mathrm{C} 180$ molecules which ciesorb and curve $b$ is for all of the $C 180$ molecules on the surface. The results shown are for simulation presented in fig. $3 \mathrm{c}$ for the case where the isotope switch is made at $\theta\left(C_{180}\right)=0.30 \ldots \ldots . .262$

Fig. 5 Variation in CO coverage versus time at $T_{s}=380 \mathrm{~K}$ based on Zhdanov's model [30]. The solid curves represent the numerical solutions of eqs. 10-13 and the symbols represent the results of Monte Carlo simulation using eq. 12 to describe $E_{d}(\theta)$. 264

Fig. 6 TPD spectra of $\mathrm{C} 180$ desorption from $\mathrm{Ni}(100)$ reported by Yates and Goodman [4]. a) Desorption in the absence of gas-phase $\mathrm{C} 16 \mathrm{O}$. For curve $\mathrm{a}$, the $\mathrm{C} 180$ exposure is $0.4 \mathrm{~L}$; for curve $b, 0.8 \mathrm{~L}$; for curve c, $1.5 \mathrm{~L}$; for curve d, $3.0 \mathrm{~L}$; and for curve e, $8.0 \mathrm{~L}$. b) Desorption of $\mathrm{C} 180$ ( $4.0 \mathrm{~L}$ dose) in the presence of gas-phase $\mathrm{C} 16 \mathrm{O}$. For curve $\mathrm{a}$, the $\mathrm{C} 16 \mathrm{O}$ flux is $0 \mathrm{~cm}^{2} \mathrm{~s}^{-1}$; for curve $b, 4.1 \times 1014 \mathrm{~cm}^{2} \mathrm{~s}-1$; for curve c, $8.3 \times 1014 \mathrm{~cm}^{2} \mathrm{~s}^{-1}$; and for curve $\mathrm{d}$, $14.5 \times 1014 \mathrm{~cm}^{2} \mathrm{~s}^{-1}$ 


\section{$\mathrm{xiii}$}

Fig. 7 Simulated TPD spectra of C18O desorption from $\mathrm{Ni}(100)$. a) Desorption in the absence of gas-phase $C 160$. For curve $a, \theta^{\circ}\left(C^{18 O}\right)=0.60$; for curve $b, 0.55$; and for curve $c, 0.25 ; b)$ Desorption of $C 180\left(\theta^{\circ}(C 18 O)\right.$ $=0.60$ ) in the presence of gas-phase $\mathrm{C} 160$. For curve a, the $\mathrm{C} 16 \mathrm{O}$ flux is $4.1 \times 1014 \mathrm{~cm}^{2} \mathrm{~s}^{-1}$; for curve b, $7.3 \times 10^{14} \mathrm{~cm}^{2} \mathrm{~s}^{-1}$; and for curve $\mathrm{c}$, $14.5 \times 1014 \mathrm{~cm}^{2} \mathrm{~s}^{-1}$

Fig. 8 a) Variation in $\theta$ T versus T. b) Variation in the average $E_{d}$ for the desorbing $C O$ molecules versus T. In both panels, curve a corresponds to a $\mathrm{C} 16 \mathrm{O}$ flux of $0 \mathrm{~cm}^{2} \mathrm{~s}^{-1}$; curve $b, 4.1 \times 1014 \mathrm{~cm}^{2} \mathrm{~s}^{-1}$; and curve $\mathrm{c}, 14.5 \times 1014 \mathrm{~cm}^{2} \mathrm{~s}^{-1}$ 


$$
\text { xiv }
$$

List of Tables

Chapter li

Table 2.1 Translational, rotational, and vibrational partitions functions for molecules [37] ...................................... 38

Table 2.2 Range of the preexponential factor from absolute

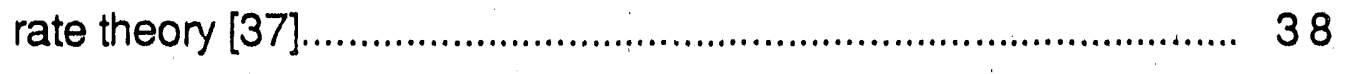

Table 3.1 Dissociative sticking probability of hydrogen on $\mathrm{Cu}$

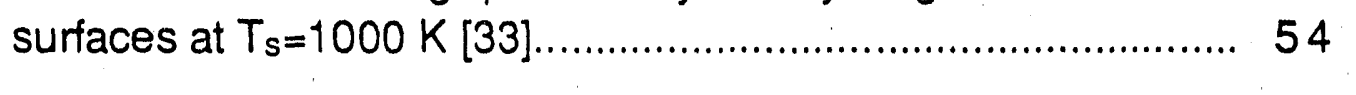

Table 3.2 Dissociative sticking probability of hydrogen on $\mathrm{Ni}$

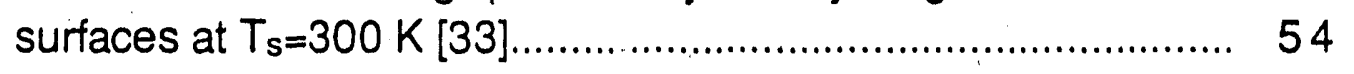

Table 3.3 Activation energy (kcal/mol) for dissociative adsorption of hydrogen and deuterium on $\mathrm{Ni}$ surfaces [55]

Table 3.4 Values of the parameters used to represent the data in fig. 3.9 [59].

Table 4.1 Arrhenius parameters for atomic self-diffusion [64]... 76

Table 6.1 Activation energy barriers (kcal/mol) for selected surface reactions determined from the BOC-MP method [17].......

Table 6.2 Parameters used to represent the CO-NO reaction network [168]....... 132

Chapter III

Table 1 Model parameters. 169 


\section{Chapter IV}

Table 1 Model parameters for the TPD simulations of associative desorption in the presence of a fixed coadsorbate.

Table 2 Model parameters for the TPD simulations of associative desorption and molecular desorption............ 228 


\section{Chapter I}

\section{Introduction}

The phenomenon of chemical reactions occurring on surfaces is the basis for a number of 'teshnological areas such as catalysis, chemical vapor deposition, and coatings. Each of these processes can be envisioned to proceed by one or more of the following elementary steps: adsorption, surface diffusion, desorption, and surface reaction. Adsorption, desorption, and surface diffusion transport adsorbates to, from, and on the surface, respectively, whereas surface reactions transform reactants to products. In order to correctly predict the kinetics of surface phenomena, an understanding of the individual and collective effects of these four elementary steps is necessary.

The earliest models for describing gas-surface interactions were based on the assumption that adsorbates interact strongly with the surface but not with each other. In many instances, though, kinetic data could not be adequately explained with this assumption. A large body of subsequent work, including present day research, has thus been devoted to studying both adsorbate-surface interactions and the lateral interactions between species on the surface. The objective of this work is to understand kinetic processes occurring on well-defined metal surfaces. Of principal interest is successfully accounting for the kinetics over a wide range of adsorbate surface concentrations.

In chapter II of this work, the theoretical models currently 
available for describing adsorption, surface diffusion, desorption, and surface reaction are reviewed. Although a large number of approaches exist for treating surface kinetics, Monte Carlo models are the most appropriate for describing the effects of lateral interactions between adsorbates over a wide range of the surface coverage. For this reason, a Monte Carlo simulation technique has been developed for describing the adsorption, surface diffusion, and desorption of adsorbates. In chapter III, the development of a Monte Carlo model for simulating the temperature-programmed desorption (TPD) of a single type of adsorbates on single-crystal metal surfaces is presented. In chapter IV, the model is extended to simulate the TPD spectra of two species coadsorbed on a surface. Finally, in chapter $V$, the influence of adsorption kinetics on the isothermal desorption and temperature-programmed desorption of a single type of species is examined. An interesting feature of the model is that it predicts kinetic behavior in good agreement with experimental observation. 


\title{
Chapter II
}

\begin{abstract}
A Review of Theoretical Models of Adsorption, Diffusion, Desorption, and Reaction of Gases on Metal Surfaces
\end{abstract}

\begin{abstract}
A review is presented of the theoretical approaches available for describing the kinetics of gas adsorption, diffusion, desorption, and reaction on metal surfaces. The prediction of rate and diffusion coefficients based on molecular dynamics, transition-state theory, stochastic diffusion theory, and quantum mechanics is discussed, and the success of these theoretical approaches in representing experimental observation is examined. Consideration is also given to the effects of lateral interactions between adsorbates and to the ability of lattice-gas models to provide a representation of the dependences of rate and diffusion coefficients on adsorbate coverage. Finally, the utility of continuum and Monte Carlo models for describing the kinetics of complex surface processes in terms of elementary processes is addressed.
\end{abstract}




\subsection{INTRODUCTION}

The kinetics of chemical reactions occurring on the surfaces of metals is of continuing interest to researchers working in the areas of catalysis, chemical vapor deposition, etching, and corrosion. While it has been known for some time that the kinetics of such reactions depend on the properties of the metal, as well as those of the adsorbed reactants, intermediates and products, it has only been in the past two decades that it has become possible to understand the dynamics of surface reactions at the atomic and molecular level. Progress has come through careful experimental ineasurements of the rates of elementary processes such as adsorption, diffusion, desorption, and reaction, and through the development of theoretical methods for predicting the dynamics of such processes. Theoretical studies have also proven useful for interpreting the experimentally observed effects of surface composition/structure anci adsorbate coverage on rate and diffusion coefficients, and for describing the kinetics of complex surface processes in terms of a sequence of elementary steps. It is therefore timely to examine the current state of the art of theoretical methods for predicting rate and diffusion coefficients for elementary processes occurring on metal surfaces and the extent to which mechanistic models are successful in representing the overall kinetics of complex surface processes (e.g., catalysis).

Previous reviews on the subjects of adsorption [1,2,3], surface diffusion [4,5], desorption [1,2,3,6,7], and surface reaction $[2,3,8]$ have been primarily concerned with summarizing experimental 
results and with presenting techniques for interpreting experimental data. While theoretical methods for predicting rate and diffusion coefficients are discussed in some of these publications, none has included a comprehensive overview of all four elementary processes. In other reviews [9-11], the theoretical approaches used to model gas-surface dynamics are described, but the success or failure of a given approach to produce results consistent with experimental observation is not treated. The purpose of this review is to put into perspective the different theoretical approaches available for describing rate and diffusion coefficients and to evaluate the success of a given approach in providing quantitative agreement with experiment. The simulation of complex surface processes in which the dynamics of individual elementary processes are represented explicitly is also discussed. To limit the scope of this review, attention is restricted to the interactions of gases with metal surfaces.

The remainder of the text is contained in Sections 2-7. Section 2 reviews the theoretical concepts used to represent rate and diffusion coefficients and discusses the relative merits and limitations of each approach. The effects of adsorbate coverage on these coefficients is also examined in this section. Sections 3-6 illustrate the success of different theoretical approaches in predicting the magnitude of the coefficients for adsorption, diffusion, desorption, and reaction, respectively. Sections 5 and 6 also discuss the simulation of temperature-programmed desorption and reaction experiments. A set of concluding remarks summarizing the present state of knowledge in gas-surface dynamics is presented 
in Section 7.

\subsection{THEORETICAL CONCEPTS}

\subsection{Adsorbate-Surface Potentials}

The dynamics of processes occurring at a metal surface are governed by the adsorbate-surface potential. This function is comprised of contributions due to metal-adsorbate interactions and lateral interactions between adsorbates. Ideally, the adsorbatesurface potential should be obtained by an accurate ab initio solution of the electronic Schrödinger equation. However, even with currently available supercomputers, it is not possible to produce complete energy hypersurfaces that are accurate enough for use in dynamical studies. As a consequence, all theoretical analyses of elementary processes occurring at surfaces are based on semiempirical expressions, the exact form of the potential depending on the nature of the problem being addressed.

Metal-Adsorbate Interactions

For weakly bonding situations between an adsorbate, $A$, and $n$ metal atoms, the metal-adsorbate interaction can be described by a sum of Lennard-Jones contributions:

$V=\sum_{i=1}^{n} 4 \varepsilon\left[\left(\frac{\sigma}{r_{i}}\right)^{12} \cdot\left(\frac{\sigma}{r_{i}}\right)^{6}\right]$

where $n$ is the separation distance between the adsorbate and the ith metal atom, and $\varepsilon$ and $\sigma$ are Lennard-Jones parameters. Where covalent bonding between the adsorbate and surface occurs, the metal-adsorbate interaction can be described by a sum of two- 
center Morse potentials

$V=V_{O A} \sum_{i=1}^{n}\left(x_{i}^{2}-2 x_{i}\right)$

$x_{i}=\exp \left[-\left(r_{i}-r_{0}\right) / a\right]$

where $V_{O A}$ is the equilibrium (minimum) potential energy, $x_{1}$ is the Pauling bond order, $r$ is the bond distance, $r o$ is the equilibrium bond distance, 'and $a$ is a scaling parameter.

More complex forms of the adsorbate-surface potential have also been used. An example is the London, Eyring, Polanyi, Sato (LEPS) potential surface, which has been used in studies of the adsorption of diatomic molecules on metal surfaces [12]. The LEPS potential for the molecule $A B$ is given by

$V=\rho_{A S}+\rho_{B S}+\rho_{A B}+\left[\chi_{A B}\left(\chi_{A B}-\chi_{A S}-\chi_{B S}\right)+\left(\chi_{A S}+\chi_{B S}\right)^{2}\right]^{1 / 2}$

where $\rho_{i j}$ and $\chi_{i j}$ are the Coulomb and exchange contributions between $i$ and $j$, and subscript $S$ denotes the surface. The contributions $p_{i j}$ and $\chi_{i j}$ are expressed in the form of modified Morse potentials.

While the adsorbate-surface potential is a multi-dimensional function in general, critical features of the potential energy surface are best characterized by examining the variation of the potential along a trajectory corresponding to the so-ualled reaction coordinate, s. Illustrations of two-dimensional potential surfaces and their one-dimensional representations corresponding to trajectories along the reaction coordinate are presented in figs. 2.1 and 2.2 for the cases of non-dissociative and dissociative adsorption, respectively.

For non-dissociative adsorption of a molecule $A B$, the one- 
dimensional potential in fig. $2.1 \mathrm{~b}$ is zero for large distances from the surface. As the adsorbate-metal distance is reduced, the potential goes through a weak minimum and then a weak maximum before it reaches the final minimum corresponding to the adsorbed state. The first minimum is associated with the presence of a weakly-bound precursor state, whereas the maximum corresponds to the activation energy barrier for adsorption. For dissociative adsorption, a qualitatively similar picture is observed. In this instance, the one-dimensional potential in fig. $2.2 \mathrm{~b}$ is described as a function of $s$, the reaction coordinate.

\section{Lateral Interactions}

While interactions between adsorbates can be ignored at low coverages, experimental evidence indicates that with increasing coverage, such interactions can become significant. Several processes can contribute to lateral interactions. Repulsive interactions can arise from the coupling of mutually aligned dipoles on the surface and from changes in adsorbate-site orbital overlap due to the interaction of multiple adsorbates with a single adsorption site. The direct interactions of orbitals on adjacent species may lead to either attractive or repulsive contributions to the total energy. The classification of an interaction between two species as purely attractive or purely repulsive, however, is not always precise. Quantum models have demonstrated that the nature of an interaction between adsorbates depends upon the separation distance and the crystallographic direction on the surface $[13,14]$.

The description of lateral interactions depends upon the nature of the system considered. For the adsorption of weakly bound 
(physisorbed) species, lateral interactions between adsorbates can be handled by the addition of appropriate Lennard-Jones terms to eq. 2.1. For strongly bound (chemisorbed) species, the effects of lateral interactions can be expressed as a perturbation in the heat of adsorption of the adsorbate in the absence of lateral interactions.

The simplest representation of lateral interactions for chemisorbed species is based on the assumption that all contributions arz painwise additive. In such a case, the heat of adsorption of species $A$ is given by $Q_{A}=Q^{0}-n W_{A A}$

where $Q^{\circ}$ is the heat of adsorption of $A$ in the absence of lateral interactions, WAA is the energy of each A-A interaction between nearest neighbors (WAA is positive for repulsive interactions and negative for attractive interactions), and $n$ is the number of nearest neighbors. The form of eq. 2.5 is derived empirically and is not based on any physical model for the interactions of nearest-neighbor adsorbates.

Recently, an alternative to eq. 2.5 has been developed based on the Bond-Order-Conservation-Morse-Potential (BOC-MP) approach pioneereo' by Shustorovich [15-17]. This methoci describes each twocenter interaction between an adsorbate atom $A$ and a surface metal atom $M$ by a Morse potential, and the total heat of adsorption is given by the sum of all two-center interactions. A further assuinption of the BOC method is that along a reaction path describing the interactions of a molecular or atomic species with a metal surface, the total bond order, $x$, is conserved and normalized to unity. 
Within the BOC-MP framework, the heat of chemisorption for an isolated atom $A$ on a surface is given by

$Q_{A, n^{\prime}}=Q_{D A}\left(2-1 / n^{\prime}\right)$

where $Q_{0 A}$ is the heat of chemisorption of $A$ in the on-top position, and $n$ ' is the number of metal atoms to which $A$ is coordinated. For an isolated molecular acisorbate $A B$ coordinated via atom $A$ to $n^{\prime}$ metal atoms, the heat of chemisorption can be approximated by $Q_{A B, n^{\prime}}=\frac{Q_{O A}^{2}}{D_{A B}+Q_{O A} / n^{\prime}}$

where $D_{A B}$ is the gas-phase $A-B$ bond energy.

Equations 2.6 and 2.7 are valid for isolated adsorbate atoms or molecules on a surface. For higher coverages, however, situations may arise in which more than one adsorbate is bonded to a metal atom and, furthermore, the adsorbates may interact directly with each other. To account for these metal-adsorbate $(M-A)$ and adsorbate-adsorbate ( $A-A)$ interactions, the total binding energy of species $A$ is partitioned as follows:

$\dot{Q}_{A, n^{\prime}}=Q_{A, n^{\prime}}^{(1)}+Q_{A, n^{\prime}}^{(2)}$

where $Q_{A, n^{\prime}}(1)$ is the heat of adsorption due to $M-A$ interactions and $Q_{A, n^{\prime}}(2)$ is the heat of adsorption due to $A-A$ interactions. Both $Q_{A, n^{\prime}(1)}$ and $Q_{A, n^{\prime}}(2)$ can be calculated explicitly as a function of the local occupancy of nearest-neighbor sites, under the constraint that the total bond order of $A$ for both $M-A$ and $A-A$ interactions is conserved to unity.

The value of $Q_{A, n^{\prime}(2)}$ can be expressed as 
$Q_{A, n^{\prime}}^{(2)}=\sum_{i=1}^{n^{\prime}} \sum_{l=1}^{L} 0.5 D_{A A}\left(2 \delta_{i l}-\delta_{i l}^{2}\right)$

where $D_{A A}$ is the A-A bond dissociation energy, and $\delta_{i l}$ is the bond order for the A-A interaction between the $A$ atom coordinated with metal $i$ and the the nearest-neighbor $A$ atom also coordinated with metal atom $i$. The summation over $l$ in eq. 2.9 is to account for all nearest-neighbor $A$ atoms. The occurrence of $A-A$ interactions weakens the bond order associated with the $M-A$ interactions and, as a consequence, the bond order for each component of an $\mathrm{Mn}^{\prime}-\mathrm{A}$ bond is given by

$x_{i, n^{\prime}}=\frac{1}{n^{\prime}} \cdot \sum_{l=1}^{L} \delta_{i l}$

The value of $Q_{A, n^{\prime}}(1)$ when more than one adsorbate is bonded to an individual metal atom is given by

$Q_{A, n^{\prime}}^{(1)}=\sum_{i=1}^{n^{\prime}}\left[\frac{Q_{0 A}}{m_{i}}\left(2-\frac{1}{m_{i}}\right)\left(2 x_{i, n^{\prime}}-x_{i, n^{\prime}}^{2}\right)\right]$

where $\mathrm{mi}_{\mathrm{i}}$ is the number of adsorbates bonded to the $i$-the metal atom, and $X_{i, n}$ is given by eq. 2.10 .

Activation Energy Barriers

The characteristic of the potential energy hypersurface having the greatest effect on the dynamics of an elementary process is the height of the activation barrier, $E$, for movement from the region of the potential surface associated with the reactant state to that associated with the product state. Since the full, multi-dimensional potential surface cannot be described in most instances, 
semiempirical models must je used to relate $E$ to the properties of the reactants and to describe the effects of lateral interactions between adsorbates.

The simplest representation of $E$ when lateral interactions occur is based on the assumption that all contributions are painwise additive. In such a case, $E$ is given by

$E=E^{\circ}-n W_{A A}$

where $E^{\circ}$ is the activation energy in the absence of lateral interactions, WAA is the contribution of nearest-neighbor A-A interactions to $E$, and $n$ is the number of nearest neighbors. The form of eq. 2.12 is empirical and is not based on any physical model for the interactions of nearest-neighbor adsorbates.

The BOC-MP [17] approach provides an alternative method for estimating the magnitude of $E$. The activation energy for dissociative adsorption from the gas phase, $E_{a}$, can be written as $E_{a}=\frac{1}{2}\left[D_{A B}-\left(Q_{A}+Q_{B}\right)+\frac{Q_{A} Q_{B}}{Q_{A}+Q_{B}}-Q_{A B}\right]$

For non-associative desorption, $E_{d}$ is given by

$E_{d}=Q_{A}$

whereas for associative desorption, $E_{d}$ is given by

$E_{d}=Q_{A}+Q_{B}-D_{A B}+E_{a} \quad$ if $E_{a}>0$

or

$E_{d}=Q_{A}+Q_{B}-D_{A B} \quad$ if $E_{a}<0$

Expressions similar to eqs. 2.15 and 2.16 can be used to calculate the activation energy for reactions, $E_{r}$. In the BOC-MP framework, the activation energy for diffusion across a bridge site, Ediff, is given by. 
$E_{\text {diff }}=\frac{\left(n^{\prime}-2\right)}{\left(4 n^{\prime}-2\right)} Q_{A}$

where $n^{\prime}$ is the coordination number for the adsorbed species in a hollow site (viz., $\left.n^{\prime}=3,4,5\right)$. The influence of lateral interactions on the activation energy can be determined using eq. 2.8 to calculate $Q_{1}$ for each species $i$ appearing in eqs. 2.13-2.17.

2.2 Kinetics of Adsorption, Desorption, and Surface Reaction

The relationship between the rate of a surface process and the adsorbate coverage-the kinetics of the process-depends on the nature of the process and the extent to which lateral interactions are important. In the absence of such interactions, and assuming that all adsorbed species are randomly distributed, simple expressions can be written to relate the rate of a process to the surface coverage, $\theta$. The dependence of the rate of elementary processes on $\theta$ are much more complex when lateral interactions are included.

Non-Interacting Adsorbates

For non-interacting adsorbates, the rate of adsorption of gasphase species, $r_{3}$, can be written as:

$r_{a}=S^{(\alpha)}(\theta) F$

where $F$ is the flux of the adsorbate and $S(\alpha)(\theta)$ is the sticking coefficient. The superscript $\alpha$ on $S(\alpha)(\theta)$ is 1 for non-dissociative and 2 for dissociative adsorption. The form of $S(\alpha)(\theta)$ depends on whether adsorption occurs directly from the gas phase or via a precursor state. For direct adsorption, 
$S^{(\alpha)}(\theta)=S^{(\alpha)}(0)(1-\theta)^{\alpha}$

where $S(\alpha)(0)$ is the sticking coefficient at zero coverage. If $S(\alpha)(0)$ is assumed to obey an Arrhenius expression, then $S^{(\alpha)}(0)=S_{0}^{(\alpha)} \exp \left(-E_{a} / k_{b} T\right)$

where $S_{0}(\alpha)$ and $E_{a}$ are the preexponential factor and activation energy for adsorption, respectively, and $k_{b}$ is the Boltzmann constant.

When adsorption is assumed to proceed via a precursor state, the mechanism of adsorption can be represented by [18]:

$$
\begin{gathered}
A \stackrel{\zeta F_{A}}{=} A^{*} \stackrel{k_{a}^{*}}{\rightarrow} A_{s} \\
\zeta F_{A_{2}} k_{a}^{*} \\
A_{2}=A_{2}^{*} \rightarrow 2 A_{s}
\end{gathered}
$$

where the precursor species for non-dissociative adsorption is denoted by $A^{*}\left(A_{2}{ }^{*}\right.$ for dissociative adsorption), the chemisorbed species by $A_{s}, \zeta$ is the trapping probability from the gas phase into the precursor state, $\mathrm{ka}^{*}$ is the rate constant for adsorption from the precursor state into the chemisorbed state, and $\mathrm{kd}^{\circ}$ is the rate constant for desorption from the precursor state. The precursor state can be located over an empty site (an intrinsic precursor) or over an occupied site (an extrinsic precursor). Two different approaches have been used to represent the adsorption rate. The 
first is based on a continuum description and uses the stationarystate approximation to determine the concentration of precursor species $[19,20]$. The second approach is based on a successive-site statistical model of the type first proposed by Kisliuk $[21,22]$. Although conceptually different, both approaches have been shown to lead to equivalent forms of the adsorption rate in many cases $[20,23]$.

If the intrinsic and extrinsic precursors are energetically equivalent and each occupies only a single adsite, then the rates of non-dissociative and dissociative adsorption can be written as [18]: $r_{a}=\frac{\zeta F_{A} \dot{k_{a}}\left(1-\theta_{A}\right)}{\dot{k}_{d}+\dot{k}_{a}\left(1-\theta_{A}\right)}$

$r_{a}=\frac{\zeta F_{A_{2}} \dot{k}_{a}\left(1-\theta_{A}\right)^{2}}{k_{d}+\dot{k}_{a}\left(\left(1-\theta_{A}\right)\right)^{2}}$

Equations 2.21 and 2.22 can be used together with eq. 2.18 to write expressions for $S(\alpha)(\theta) / S(\alpha)(0)$. Thus,

$S^{(1)}(\theta) / S^{(1)}(0)=\frac{(1+K)\left(1-\theta_{A}\right)}{1+K\left(1-\theta_{A}\right)}$

$S^{(2)}(\theta) / S^{(2)}(0)=\frac{(1+K)\left(1-\theta_{A}\right)^{2}}{1+K\left(1-\theta_{A}\right)^{2}}$

where $K=K_{a} / K_{d^{*}}$. A plot of $S(1)(\theta) / S(1)(0)$ versus $\theta$ is shown in fig. 2.3. When $K>1, S(1)(\theta) / S(1)(0)=1$ and when $K \ll 1, S(1)(\theta) / S(1)(0)=$ $(1, B)$. Since the value of $K$ is temperature dependent, the shape of $S(\alpha)(\theta) / S(\alpha)(0)$ versus $\theta$ will depend on temperature. 
The rate of desorption for a randomly distributed adsorbate in the absence of lateral interactions can be written as

$r_{d}=k_{d}^{(\alpha)} \theta^{\alpha}$

where $k_{d}(\alpha)$ is the rate coefficient for desorption. If $k_{d}(\alpha)$ can be described by an Arrhenius expression, then

$k_{d}^{(\alpha)}=v_{d}^{(\alpha)} \exp \left(-E_{d} / k_{b} T\right)$

where $v_{d}(\alpha)$ and $E_{d}$ are the preexponential factor and activation energy for desorption, respectively.

Implicit in the formulation of eq. 2.26 is the assumption that desorption occurs directly from the adsorbed state. This assumption is unnecessarily restrictive since it is conceivable that the adsorbate passes through a weakly bound precursor state before leaving the catalyst surface. Making the same assumptions used in deriving eqs. 2.21 and 2.22, the rates of non-associative and associative desorption can be written as [18]:

$$
\begin{aligned}
& r_{d}=\frac{k_{d}^{(1)} \dot{k}_{d} \theta_{A}}{k_{a}\left(1-\theta_{A}\right)+\dot{k}_{d}^{\dot{0}}} \\
& r_{d}=\frac{k_{d}^{(2)} \dot{k}_{d} \theta_{A}^{2}}{k_{a}\left(1-\theta_{A}\right)^{2}+\dot{k}_{d}^{*}}
\end{aligned}
$$

In the limit $k_{a^{*}}(1-\theta)^{\alpha} \ll k_{d^{*}}$, eqs. 2.27 and 2.28 reduce to eq. 2.25 .

Surface reactions can be classified into two generic types.

The first includes reactions between two adsorbed species or an adsorbed species and a vacant site (Langmuir-Hinshelwood processes). For randomly distributed adsorbates on a surface in the 
absence of adsorbate-adsorbate interactions, the rate of reaction is given by:

$r_{r}=k_{r} \theta_{A} \theta_{B}$

or

$r_{r}=k_{r} \theta_{A} \theta_{V}$

where $k_{r}$ is the rate coefficient, $\theta_{l}$ is the surface coverage of species $i$ and $\theta V$ is the fraction of vacant sites. If $k_{r}$ follows an Arrhenius expression, then

$k_{r}=v_{r} \exp \left(-E_{r} / k_{b} T\right)$

where $v_{r}$ and $E_{r}$ are the preexponential factor and the activation energy for reaction, respectively.

The second class of reactions includes the direct interaction of a gas-phase species with an adsorbed species to form a product which may either remain adsorbed or desorb into the gas phase (EleyRideal processes). For such processes, the rate of reaction can be written as

$r_{r}=k_{r} \theta_{A} P_{B}$

where $P_{B}$ is the partial pressure of reactant $B$. The form of eq. 2.32 is similar to that for adsorption and so $k_{r}$ can be represented as a reactive sticking coefficient, So, by the expression

$k_{r}=\frac{S_{o} a_{S}}{\left(2 \pi m_{B} k_{b} T_{g}\right)^{1 / 2}} \exp \left(-E_{r} / k_{b} T\right)$

where as is the area per reaction site, and $m_{B}$ is the molecular weight of species $B$.

Interacting Adsorbates

When lateral interactions become significant, the relationships between the rate of an elementary process and the 
adsorbate coverage become quite complex and cannot, in general, be written in closed form. An exception to this occurs in the case where a lattice-gas model is used to describe the effects of lateral interactions. In such a model, each adsorbate is assumed to be localized on a two-dimensional array of surface sites, and each site is assumed to be either vacant or occupied by a single adsorbate. A given adsorbate can interact with adsorbates on nearest-neighbor sites, next-nearest-neighbor sites, etc., but in most variants of the lattice-gas model, only nearest-neighbor interactions are taken into account. Using these assumptions, relationships can be derived between the adsorbate coverage and the rate of adsorption, desorption, and surface reaction [24-26].

The rate of non-dissociative adsorption is defined as $r_{a}=F S_{0}^{(1)} \sum_{n} P_{V, n} \exp \left[\frac{-\left(E_{a}^{0}-E_{s}\right)}{k_{b} T}\right](1-\theta)$

where $S_{0}(1)$ is the initial sticking coefficient, $P V, n$ is the probability of finding a vacant site with $n$ occupied nearest-neighbor sites, $E_{a}{ }^{\circ}$ is the activation energy for adsorption in the absence of nearestneighbor interactions, and $E_{s}$ is the interaction energy of $A$ with its neighbors. Es can be related to the energy for a given pairwise interaction, $W A A$, by $E_{S}=n W A A$. For dissociative adsorption, the rate of adsorption can be expressed as

$r_{a}=F S_{0}^{(2)} \sum_{n, m} P_{V V i n m} \exp \left[\frac{-\left(E_{a}^{0}-E_{S}\right)}{k_{b} T}\right]$

where PVVinm is the probability that two nearest-neighbor sites are vacant such that one is surrounded by $n$ nearest neighbors and the other is surrounded by $m$ nearest neighbors. 
To complete the description of the lattice-gas model, it is necessary to develop expressions for $P V, n$ and $P V V ; n m$ in terms of $\theta_{A}$ and WAA. Since an exact solution to this problem can only be achieved for a coverage of 0.5 , an approximation must be made to obtain a solution in closed form. One of the more widely used postulates is the quasi-chemical approximation (QCA) which assumes that the adsorbates maintain an equilibrium Jistribution on the surface. The QCA treats the probabilities $P V, n$ and $P V V ; n m$ in terms of the probabilities $P_{A A}, P_{A V}$, and $P_{V V}$, where $P_{A A}$ is the probability that two nearest-neighbor sites are occupied by species $A, P \vee v$ is the probability that two nearest-neighbor sites are vacant, and $P_{A V}$ is the probability that of two nearest-neighbor sites, one is occupied and the other is vacant. These probabilities are described by the equations:

$$
\begin{aligned}
& P_{A A}+P_{A V}+P_{V V}=1 \\
& 2 P_{A A}+P_{A V}=2 \theta \\
& \frac{P_{A A} P_{V V}}{P_{A V}^{2}}=\frac{1}{4} \exp \left[\frac{-W_{A A}}{k_{b} T}\right]
\end{aligned}
$$

The solution to eqs. $2.36-2.38$ is given by

$$
\begin{aligned}
& P_{A A}=\theta-\left\{1-[1-2 \eta \theta(1-\theta)]^{1 / 2}\right\} i \eta \\
& P_{A V}=2\left\{1-[1-2 \eta \theta(1-\theta)]^{1 / 2}\right\} / \eta \\
& P_{V V}=1-\theta-\left\{1-[1-2 \eta \theta(1-\theta)]^{1 / 2}\right\} / \eta \\
& \text { where } \eta=2\left[1-\exp \left(-W_{A A} / k_{b} T\right)\right] .
\end{aligned}
$$

In the QCA, different pairs of sites are considered to be independent and hence 
$P_{V, n}=\frac{z !}{n !(z-n) !} \frac{\left(0.5 P_{A V}\right)^{n}\left(P_{V V}\right)^{z-n}}{\left(0.5 P_{A V}+P_{V V}\right)^{z}}$

where $z$ is the number of nearest-neighbor sites. Using eqs. 2.392.42, the following functions can be defined to represent the sums appearing in eqs. 2.34 and 2.35, respectively [25]:

$$
\begin{aligned}
f_{a}^{\prime}\left(\theta, w_{A A}\right) & =\sum_{n} P_{V, n} \exp \left[\frac{n w_{A A}}{k_{b} T}\right] \\
& =\left(\frac{0.5 P_{A V} \exp \left[\frac{w_{A A}}{k_{b} T}\right]+P_{V V}}{1-\theta}\right)^{2}
\end{aligned}
$$

$f_{a}{ }^{\prime \prime}\left(\theta, w_{A A}\right)=\sum_{n, m} P_{V V ; n m} \exp \left[\frac{(n+m) w_{A A}}{k_{b} T}\right]$

$$
=\operatorname{PVV}\left(\frac{0.5 P_{A V} \exp \left[\frac{W_{A A}}{k_{b} T}\right]+P_{V V}}{1-\theta}\right)^{2 z-2}
$$

When WAA $=0, f_{a}{ }^{\prime}\left(\theta_{A}, W A A\right)=1$ and $f_{a}{ }^{\prime \prime}\left(\theta_{A}, W A A\right)=(1-\theta)^{2}$, and eqs. 2.34 and 2.35 become identical to eqs. 2.18-2.20. Thus, $f_{a}{ }^{\prime}$ and $f_{a}{ }^{\prime \prime}$ represent corrections to eqs. $2.18-2.20$ due to lateral interactions.

The rate of non-associative desorption is defined as $r_{d}=v_{d}^{(1)} \sum_{n} P_{A, n} \exp \left[\frac{-\left(E_{d}^{0}-E_{s}\right)}{k_{b} T}\right] \theta$

where $P_{A, n}$ is the probability of finding an adsorbate $A$ with $n$ nearest-neighbors, $E_{d}{ }^{\circ}$ is the activation energy for desorption in the absence of nearest-neighbor interactions, and $E_{s}$ is the interaction energy of $A$ with its neighbors. $E_{s}$ can be related to the energy for a given pairwise interaction, $W A A$, by $E_{s}=$ nWAA. For associative desorption, the rate of desorption can be expressed as 
$r_{d}=v_{d}^{(2)} \sum_{n, m} P_{A A ; n, m} \exp \left[\frac{-\left(E_{d}^{0}-E_{S}\right)}{k_{b} T}\right]$

where $P_{A A}, n, m$ is the probability that two nearest-neighbor sites are occupled by $A$ atoms such that one is surrounded by $n$ nearest neighbors and the other is surrounded by $m$ nearest neighbors.

Expressions for $P_{A, n}$ and $P_{A A ; n, m}$ can be written using the $Q C A$.

Thus, for $P_{A, n}$ one can write

$P_{A, n}=\frac{z !}{n !(z-n) !} \frac{P_{A A}^{n}\left(0.5 P_{A V}\right)^{z-n}}{\left(P_{A A}+0.5 P_{A V}\right)^{z}}$

Using eqs. 2.39-2.41 and 2.47, one can define the following functions $[24,26]$ :

$f_{d}^{\prime}\left(\theta, w_{A A}\right)=\sum_{n} P_{A, n} \exp \left[\frac{n w_{A A}}{k_{b} T}\right]$

$$
=\left(\frac{P_{A A} \exp \left[\frac{W_{A A}}{k_{b} T}\right]+0.5 P_{A V}}{\theta}\right)^{2}
$$

$f_{d}^{\prime \prime}\left(\theta, w_{A A}\right)=\frac{\sum_{n, m} P_{A A ; n, m} \exp \left[\frac{(n+m) w_{A A}}{k_{b} T}\right]}{\theta^{2}}$

$$
=P_{A A}\left(\frac{P_{A A} \exp \left[\frac{W_{A A}}{k_{D} T}\right]+0.5 P_{A V}}{\theta}\right)^{2 z \cdot 2}
$$

Substitution of eqs. 2.48 and 2.49 into eqs. 2.45 and 2.46 , respectively, gives:

$r_{d}=v_{d}^{(1)} \exp \left[\frac{-E_{d}^{0}}{k_{b} T}\right] \theta f_{d}^{\prime}\left(\theta, w_{A A}\right)$ 
$r_{d}=v_{d}^{(2)} \exp \left[\frac{-E_{d}^{0}}{k_{b} T}\right] f_{d}^{\prime \prime}\left(\theta, w_{A A}\right)$

When $W_{A A}=0, f^{\prime}\left(\theta_{A}, W_{A A}\right)=1.0$ and $f_{d}{ }^{\prime \prime}\left(\theta_{A}, W_{A A}\right)=\theta_{A}{ }^{2}$, and eqs. 2.50

and 2.51 become identical to eqs. 2.25 and 2.26. Thus, $\mathrm{fd}^{\prime}$ and $\mathrm{fd}_{\mathrm{d}}{ }^{\prime \prime}$ represent corrections to the Polanyi-Wigner expressions for desorption (eqs. 2.25 and 2.26) due to lateral interactions. The functions $\mathrm{fd}^{\prime}$ and $\mathrm{fd}^{\prime \prime}$ are plotted in fig. 2.4 for attractive and repulsive values of $W_{A A} / k_{b} T$. It is apparent that even for small values of $W_{A A} / k_{b} T$, lateral interactions can have a profound effect on $f^{\prime}$ and $f_{d \prime}{ }^{\prime \prime}$.

Another way of visualizing the effects of lateral interactions is to look at the coverage dependencies of the apparent activation energy and the apparent preexponential factor. "For non-associative desorption, these quantities are defined by [26]:

$\Delta E_{d}(\theta)=k_{b} T^{2} \frac{d \ln f_{d}}{d T}$

$\frac{v_{d}^{(1)}(\theta)}{v_{d}^{(1)}(0)}=f_{d}^{\prime} \exp \left[\frac{\Delta E_{d}(\theta)}{k_{b} T}\right]$

Figure 2.5 shows that the variation in $\Delta E_{d}(\theta)$ and $v_{d}(1)(\theta) / v_{d}(1)(0)$ with $\theta$ is a strong function not only of WAA/kbT, but also of $z$. The apparent activation energy is seen to vary monotonically with coverage whereas the apparent preexponential factor exhibits a more complex coverage dependence.

The lattice-gas model developed for the description of associative desorption can be extended to describe the kinetics of 
Langmuir-Hinshelwood processes. The rate of the bimolecular reaction $A_{s}+B_{s} \rightarrow C_{s}$ or $C_{g}$ can be written as $r_{r}=v_{r} \sum_{i} P_{A B ; i} \exp \left[\frac{-\left(E_{r}^{0}-E_{s}\right)}{k_{b} T}\right]$

where $v_{r}$ is the preexponential factor, $P_{A B ; i}$ is the probability that two nearest sites are occupied by an $A B$ pair and that this pair has the environment $i, E_{r}{ }^{\circ}$ is the activation energy for the reaction of $A$ and $B$ in the absence of nearest-neighbor interactions, and $E_{s}$ is the contribution to the activation energy due to nearest-neighbor interactions. The lateral interaction energy of the $A B$ pair is determined by $k, l, k^{\prime}$, and $I^{\circ}$, where $k$ and $I\left(k^{\circ}\right.$ and $\left.I^{\prime}\right)$ are the numbers of $A$ and $B$ neighbors of $A_{s}\left(B_{s}\right)$, respectively. Using these indices, $E_{s}$ is written as

$E_{s}=k w_{A A}-\left(1+k^{\prime}\right) w_{A B}-l^{\prime} w_{B B}$

In the QCA, the probability $P_{A B ; i}$ can be expressed in terms of $P_{A A}, P_{A B}, P_{B B}, P_{A V}, P_{B V}, P_{V V}$, and $P_{A B}$. These probabilities are described by the equations

$$
\begin{aligned}
& P_{A A}+P_{A B}+P_{A V}+P_{B B}+P_{B V}+P_{V V}=1 \\
& 2 P_{A A}+P_{A B}+P_{A V}=2 \theta_{A} \\
& 2 P_{B B}+P_{A B}+P_{B V}=2 \theta_{B} \\
& \frac{P_{A A} P_{V V}}{P_{A V}^{2}}=\frac{1}{4} \exp \left(-w_{A A} / k_{D} T\right) \\
& \frac{P_{B B} P_{V V}}{P_{B V}^{2}}=\frac{1}{4} \exp \left(-w_{B B} / k_{D} T\right)
\end{aligned}
$$

$$
\frac{P_{A A} P_{B B}}{P_{A B}^{2}}=\frac{1}{4} \exp \left[\frac{-\left(W_{A A}+W_{B B}-2 W_{A B}\right)}{k_{b} T}\right]
$$


Zhdanov [24,25] has shown that within the QCA, $\mathrm{rr}$ can be written as

$r_{r}=v_{r} \exp \left[\frac{-E_{r}^{0}}{k_{b} T}\right] P_{A B}\left(\frac{P_{A A} \exp \left[W_{A A} / k_{b} T\right]+0.5 P_{A B} \exp \left[W_{A B} / k_{b} T\right]+0.5 P_{A V}}{\theta_{A}}\right)^{z-1} x$

$\left(\frac{P_{B B} \exp \left[W_{B B} / k_{b} T\right]+0.5 P_{A B} \exp \left[W_{A B} / k_{b} T\right]+0.5 P_{B V}}{\theta_{B}}\right)^{2-1}$

It is apparent from eq. 2.62 that inclusion of interactions between species leads to a rate expression dependent on the sign and magnitude of these interactions. Moreover, the dependence of $\mathrm{rr}$ on $\theta_{A}$ and $\theta_{B}$ is seen to differ significantly from that based on the assumption of a random adsorbate distribution, as described by eq. 2.29. To establish the extent to which eqs. 2.29 and 2.62 differ, it is useful to define the function [25]

$f_{r}=\frac{\sum_{i} P_{A B ; i} \exp \left[\frac{\Delta E_{r}(\theta)}{k_{b} T}\right]}{\theta_{A} \theta_{B}}$

Calculations of $f_{r}$ for a square lattice are given in fig. 2.6. Both the magnitude and sign of the $A-A, A-B$, and $B-B$ interactions influence $f_{r}$.

\subsection{Diffusion}

The diffusion of an adsorbate on a surface can be characterized by two types of motion. When the activation energy for diffusion, 
$E_{\text {diff, }}$ is less than $k_{b} T$, the adsorbate translates freely across the surface. This type of motion, which is termed mobile diffusion, is characteristic oi physisorbed species. When Ediff is significantly greater than $\mathrm{kbT}$, the adsorbate moves from site to site by hopping over the activation barrier. Diffusion via a hopping mechanism is characteristic of chemisorbed species.

In the presence of a concentration gradient, the diffusive flux, $\mathrm{J}$, of an adsorbate is given by Fick's law:

$J=-D \nabla C$

where $C$ is the concentration of adsorbate and $D$ is the concentrationindependent diffusivity. For a freely mobile adsorbate, the diffusivity can be expressed as:

$D=\frac{1}{d} \bar{v} \lambda$

where $\bar{v}$ is the mean velocity of the adsorbate, $\lambda$ is the mean-free path, and $d$ is the number of dimensions in which the motion occurs ( $d=2$ for an isotropic surface). For hopping motion, the diffusivity is written as

$D=\frac{1}{2 d} \Gamma \lambda^{2}$

where $\Gamma$ is the hopping frequency and $\lambda$ now represents the meanfree hopping length. Since hopping is an activated process which is known to depend on temperature, it is customary to express $\Gamma$ as $\Gamma=v_{\text {diff }} \exp \left(-E_{\text {diff }} / k_{b} T\right)$

where $v$ diff is the preexponential factor for hopping. Substitution of eq. 2.67 into eq. 2.66 leads to:

$D=\frac{1}{2 d} \lambda^{2} v_{\text {diff }} \exp \left(-E_{\text {diff }} / k_{b} T\right)$ 
$=D_{0} \exp \left(-E_{\text {diff }} / k_{b} T\right)$

where $D_{0}$ is referred to as the preexponential factor for diffusion.

\subsection{Theoretical Descriptions of Surface Dynamics}

Theoretical descriptions of the dynamics of elementary processes occurring on metal surfaces have been developed on the basis of molecular dynamics, transition-state theory, absolute rate theory, stochastic diffusion theory, and quantum mechanics. Each of these approaches is reviewed to illustrate the manner in which rate or transport coefficients are related to the adsorbate-surface potential.

\section{Molecular Dynamics}

The motion of an adsorbate near or on a surface can be obtained from molecular dynamics (MD) simulations. Such simulations provide a spatial and temporal description of the adsorbate trajectory, which can, in turn, be used to determine the sticking coefficient and the surface diffusion coefficient. For reasons discussed below, MD simulations are not well suited, in most cases, for determining the rate coefficient for desorption.

If the atoms in the metal surface are assumed to be stationary, then the adsorbate trajectory, $Y(t)$, is given by $m \ddot{Y}=-\partial V(Y) / \partial Y$

where $m$ is the adsorbate mass and $V(Y)$ is the adsorbate-surface potential. A more reailistic description of adsorbate-surface interactions is obtained when the metal atoms are allowed to vibrate. Treating the motion of all the metal atoms, however, is 
computationally infeasible. To circumvent this, the problem is formulated in the following manner [27]. The adsorbate is assumed to interact strongly with $n(1-10)$ atoms of the metal. This cluster constitutes the primary zone, and the interactions between the adsorbate and the metal atoms in the primary zone is described by $V_{p}\left(X_{p}, Y\right)$, where $Y$ describes the position of the adsorbate and $X_{p}$ describes the positions of the metal atoms in the primary zone. Adsorbate interactions with metal atoms outside the primary zone (i.e., in the secondary zone or heat bath) are described by the potential $V_{s}(\mathbf{Y})$. Thus the total interaction potential is $V\left(x_{p}, \mathbf{Y}\right)=$ $\dot{V}_{s}(Y)+V_{p}\left(X_{p}, Y\right)$. The motion of the adsorbate is given by $m \ddot{Y}=-\partial V\left(X_{p}, Y\right) / \partial Y$

whereas the motion of the metal atoms in the primary zone is given by a generalized Langevin equation

$m^{\prime} \ddot{x}_{p}=-\omega_{p p}^{2} m^{\prime} x_{p}-\partial V\left(x_{p}, Y\right) / \partial x_{p}+m^{\prime} \int_{0}^{1} \Theta(t-\tau) x_{p} d \tau+R(t)$

where $\mathrm{m}^{\prime}$ is the mass of metal atom. The parameter $\omega_{p p}$ in eq. 2.71 is the characteristic frequency for vibration of metal atoms in the primary zone, and the functions $\Theta(t)$ and $\mathbf{R}(t)$ correspond to a memory kernel and a random force. The integral involving $\Theta(t)$ describes the dissipation of energy from the primary zone to the heat bath, whereas $\mathbf{R}(t)$ describes the transfer of energy from the heat bath to the primary zone. The last two terms in eq. 2.71 satisfy the second fluctuation-dissipation theorem, so that the surface temperature remains constant.

The solution of eq. 2.69 or eqs. 2.70 and 2.71 gives $Y(t)$, the 
trajectory of the adsorbate as a function of time. The probability of adsorption can be determined by examining a collection of trajectories calculated for different initial conditions. Since the probability of adsorption is related to the time scale of the observation (i.e., a species which adsorbs may desorb at a later time), a working definition of adsorption must be adopted. As an example of such a definition, an adsorbate can be assumed to be trapped and equilibrated with the surface if at some time in its history the total energy of the adsorbate on the surface becomes less than $-3 k_{b} T[28,29]$. Alternatively, one can assume that adsorption occurs when the adsorbate-surface separation distance is less than some prescribed value. Thus, the fraction of all adsorbate trajectories satisfying the working definition condition can be defined as the sticking coefficient for non-dissociative adsorption, $S(1)(T)$. For dissociative adsorption of a molecule $A_{2}$, the fraction of all trajectories in which the A-A bond distance exceeds a specified value at some time is defined as $S(2)(T)$ [30-35].

MD simulations can also be used to predict the diffusion coefficient of an adsorbate on a surface. For mobile diffusion, $D$ is defined by the Einstein relationship as

$D=\left\langle[Y(t)-Y(0)]^{2}>14 t\right.$

where $<[Y(t) \cdot Y(0)]^{2}>$ represents the ensemble average of the meansquare displacement.

The use of MD simulations to describe the dynamics of desorption and surface reactions is limited by technical difficulties. The numerical algorithms used for the solution of eq. 2.69 or eqs. 2.70 and 2.71 require integration step lengths comparable with the 
time scale of the fastest motion, usually a vibrational period of $10.14 \mathrm{~s}$. While simulation of processes which occur on the 1 to 103 picosecond time scale is practical, direct simulation of slow events which occur over microseconds or longer is usually prohibitive. In the case of desorption and surface reaction, the trajectory of the adsorbate must pass through a narrow region of phase space (i.e., the region of the col on the potential surface). Because such events occur infrequently, very long integration times are required if $E_{d} \gg$ $k_{b} T$, and consequently, direct simulation of such events is impractical. As discussed below, the description of infrequent events is best handled by a dynamical form of transition-state theory.

\section{Transition-State Theory}

In classical transition-state theory (TST), the rate for a species goirig from state $A$ to state $B, A \rightarrow B$; is defined as the equilibrium flux of adsorbate trajectories across a plane in phase space. The plane, $S$, which lies between the reactants and products, must be traversed at least once in going from $A$ to $B$. While the location of the plane is arbitrary, it is often convenient to specify the location of $S$ near or at the col on the potential energy surface (i.e., the transition state). Since some trajectories of species $A$ may make multiple crossings of $S$ before finally going to $B$ whereas other trajectories which cross $S$ correspond to the processes $A \rightarrow A$ or $B$ $\rightarrow B$, simply counting adsorbate trajectories leads to an overestimation of the rate. Thus, TST provides an upper limit to the true rate. The derivation of the TST formalism presented in the balance of this section is based on the work of Tully and coworkers $[28,36]$. 
For the process $A \rightarrow B$, the TST equilibrium rate coefficient, $k$, is given by [28]

$$
k=\frac{\int d p \int d q \int_{0} d v_{s} p\left(p, q, s_{0}, v_{s}\right) v_{s} \xi\left(p, q, v_{s}\right)}{\int d p \int d q \int_{-}^{\infty} d v_{s} \int_{-\infty}^{\infty} d s p\left(p, q, s, v_{s}\right)}
$$

where the vectors $q$ and $p$ denote position and momenta, respectively, $s$ is the coordinate normal to the counting plane located at so, and $V_{s}$ is the component of velocity in the $s$ direction. Reactant $A$ is located in regions of phase space where $s<s_{0}$; conversely, product $B$ is located in regions of phase space where $\mathbf{s}>$ so. The equilibrium probability density function in phase space is defined as

$P\left(p, q, s, v_{s}\right)=N \exp \left(-H\left(p, q, s, v_{s}\right) / k_{b} T\right)$

which is valid for a canonical ensemble. Here, $H$ is the the classical Hamiltonian of the system and $\mathrm{N}$ is a normalization constant. The factor $\xi\left(p, q, v_{s}\right)$ in eq. 2.73 is used to correct for trajectories which make multiple crossings of $S$ or which do not contribute to the process $A \rightarrow B$.

It is convenient to factor eq. 2.73 into [28] $k=k T S T f_{s}$

where $\mathrm{kTST}$ is the uncorrected equilibrium rate constant given by 


$$
k_{T S T}=\frac{\int d p \int d q \int_{0}^{-} d v_{s} p\left(p, q, s_{0}, v_{s}\right) v_{s}}{\int d p \int d q \int_{-}^{-} d v_{s} \int_{-}^{w_{0}} d s p\left(p, q, s, v_{s}\right)}
$$

and $f_{s}$ is the dynamic correction term given by

$$
f_{s}=\frac{\int d p \int d q \int_{0} d v_{s} p\left(p, q, s_{0}, v_{s}\right) v_{s} \xi\left(p, q, v_{s}\right)}{\int d p \int d q \int_{0}^{-} d v_{s} P\left(p, q, s, v_{s}\right)}
$$

For a canonical ensemble where $H$ is given as the sum of kinetic and potential energy terms, eq. 2.76 can be reduced to

$$
k_{T S T}=\left(\frac{k_{b} T}{2 \pi m}\right)^{1 / 2} \frac{\int d q \exp \left[-V(q, s) / k_{b} T\right]}{\int d q \int_{-}^{\infty} d s \exp \left[-V(q, s) / k_{b} T\right]}
$$

where $\left(k_{b} T / 2 \pi m\right)^{1 / 2}$ is the mean velocity.

Equation 2.78 can be rewritten in terms of a one-dimensional potential of mean force, $W(s)$, defined as [36]

$W(s)=-k_{b} T \ln [g(s) / g(\infty)]$

$g(s)=G^{-1} \int d q \exp \left[-V(q, s) / k_{b} T\right]$

where $G$ is an arbitrary normalization factor. Substitution of eqs. 
2.79 and 2.80 into eq. 2.78 leads to

$$
k_{T S T}=\left(\frac{k_{b} T}{2 \pi m}\right)^{1 / 2} \frac{\exp \left[-W(s) / k_{b} T\right]}{\int_{-}^{\infty} d s \exp \left[-W(s) / k_{b} T\right]}
$$

Thus, the multi-dimensional potential $V(q, s)$ appearing in eq. 2.78 can be represented in the one dimension of the reaction coordinate, S.

In calculating $k$ from eq. 2.73 , the product $k T S T f_{s}$ is independent of the location of $S$, but the individual terms KTST and $f_{s}$ are not. The most suitable position for $S$ depends on the rate process under investigation. For desorption, placement of $S$ far enough away from the surface so that adsorbate-surface interactions are negligible allows is to be equated with the thermally-averaged sticking coefficient. For diffusion, the location for $S$ is specified such that irajectories crossing the bridge sites between two surface atoms are normal to $S$. An alternative approach for locating $S$ is used in variational transition-state theory (VTST). In this approach, the position of $S$ is varied to obtain to the minimum value of KTST, i.e., the closest approximation to $k$. The principle consequence of using VTST is that the effect of multiple crossings of the potential barrier is minimized. From eq. 2.81, it is evident that the VTST location of $S$ corresponds to the maximum value of $W(S)$.

A number of techniques exist to determine the recrossing factor is. An exact approach is to calculate $f_{s}$ by forward and backward integration of trajectories initiated at the dividing surface $S$. Approximations to fs have also been proposed. One 
example is the unified statistical model which predicts that is is given by [36]

$$
f_{s}=\frac{\exp \left[W^{\dagger} / k_{b} T\right]}{\left\{\exp \left[W^{\dagger} / k_{b} T\right]+\exp \left[W^{\dagger} / k_{b} T\right]-\exp \left[W^{*} / k_{b} T\right]\right\}}
$$

where $W+$ is the higher maximum in the potential of mean force, Wtt is the lower maximum, and $W^{*}$ is the minimum that lies between the two maxima.

The unifled statistical model also provides an approximation for the sticking coefficient $S(\alpha)(T)$ for adsorption into the chemisorbed state [36]

$S^{(\alpha)}(T)=\left\{1+\exp \left[W^{\prime} / k_{b} T\right]-\exp \left[W^{*} / k_{b} T\right\}^{-1}\right.$

$W I$ is the potential of mean force at the inner barrier for adsorption. If a secondary maximum or minimum does not exist, then the corresponding potential of mean force $W^{\prime}$ or $W^{*}$ is simply set equal to zero in eq. 2.33 .

Dynamically corrected transition-state theory can also be used to determine the diffusion coefficient for chemisorbed species. In this case, hopping of the adsorbate is treated as if it were a reaction moving the adsorbate from site $i$ to site $j$. The hopping frequency $\Gamma_{i j}$ is then given by

$\Gamma_{i j}=k_{T S T} f_{s}$

and the diffusion coefficient is related to $\Gamma_{\|}$by $D=\frac{\lambda^{2}}{4} \Gamma_{1 j}$

\section{Absolute Rate Theory}

It is evident from the above discussion that the evaluation of 
KTST from eq. 2.78 requires a knowledge of $V(q, s)$. If an accurate description of $V(q, s)$ is not available, as is most often the case, then KTST can be estimated using absolute rate theory as

$k_{T S T}=\frac{k_{b} T}{h} \frac{q^{\dagger}}{q_{0}} \exp \left[-\left(E^{\dagger}-E_{0}\right) / k_{b} T\right]$

where qt and $q_{0}$ are the partition functions for the transition state and the reactant, Et and $E_{0}$ are the energies of the transition state and the reactant, and $h$ is Planck's constant.

To evaluate kTST using eq. 2.86, estimates must be made of q and $q_{0}$. An accurate determination of $q_{0}$ is possible only if the reactant is in the gas phase. For a reactant in the adsorbed state, $q_{0}$ can be estimated provided some reasonable assumptions of the reactant structure and mobility can be made. Estimation of qt is much more difficult since the exact nature of the transition-state complex is unknown, and hence, its structure and mobility can not be defined with any accuracy. Nevertheless, it has been found [37-40] that reasonable estimates of the preexponential factor in KTST can be made using eq: 2.86. Formulas for calculating the contributions to $\mathrm{q}^{t}$ and $q_{0}$ due to translational, rotational, and vibrational degrees of freedom are given in table 2.1. Also indicated in table 2.1 are the magnitudes of each partition function for four species. In table 2.2 are shown the range of values for preexponential factors which can be accounted for by absolute rate theory. It is apparent that for some surface processes, the preexponential factor can fall in a range which spans several orders of magnitude.

In absolute rate theory, the electronic contributions to the partition functions of the reactant and transition-state complex are 
factored out and expressed in the exponential factor appearing in eq. 2.86. The difference between the electronic energy states of the transition complex and the reactant, $\left(E t-E_{0}\right)$, is defined as the activation energy, E. Since absolute rate theory does not provide a means for determining $E$, this parameter must be estimated using one of the methods presented in Section 2.1.

Stochastic Diffusion Theory

Goddard and coworkers [41] have developed a theory for the desorption of atoms and molecules from surfaces based on classical stochastic diffusion theory. This approach uses a one-dimensional representation of the adsorbate-surface interaction. The flux of desorbing atoms or molecules across a plane parallel to the surface and located at so is given by

$F\left(s_{0}, u_{0}\right)=\int_{\infty}^{\infty} d u P\left(s_{0}, u\right) u$

where $P\left(S_{0}, U\right)$ is the probability of finding the adsorbate at $S=s_{0}$ with velocity $u_{1}$ and $u_{0}$ is the smallest (positive) velocity for which a particle at so will desorb. $P\left(S_{0}, u\right)$ is determined by solving the generalized Liouville equation. The effective force acting on the adsorbate and the friction constant for energy dissipation are deduced from a representation of the adsorbate-surface interactions using a generalized Langevin equation (see eqs. 2.70-2.71). The final expression for $k_{d}(1)$ in the case of atomic desorption is

$k_{d}^{(1)}=\frac{\Omega_{0}}{2 \pi} \exp \left[-E_{d} / k_{b} T\right]$

where $\Omega_{0}$ is the frequency of the vibration for the adsorbate at the 
bottom of the adsorbate-surface potential well and $E_{d}$ is the apparent activation energy for desorption

$E_{d}=V\left(s_{0}\right)+1 / 2 m u_{0}^{2}$

For molecular desorption, $k d^{\prime}(1)$ is given by

$k_{d}^{(1)}=\frac{\Omega_{0}}{2 \pi}\left(\frac{2 \mu I^{2} \Omega_{r}^{2} \gamma_{0}^{2}}{\pi k_{b} T}\right) \exp \left[-E_{d} / k_{b} T\right]$

where $\mu$ is the reduced mass for frustrated rotational motion of the molecule, $I$ is the effective length of the molecule, $\Omega r$ is the rotational frequency, and $\gamma_{0}$ is the maximum bending angle for the molecule. Since the molecule has rotational modes, Ed for molecules must be modified to:

$E_{d}=V\left(s_{0}\right)+1 / 2 m u_{0}^{2}+1 / 2 \mu l^{2} \dot{\gamma}^{2}+1 / 2 \mu l^{2} \Omega_{r}^{2} \gamma^{2}$

where $\gamma$ is the bending angle.

\section{Quantum Mechanics}

Quantum mechanical treatments of the dynamics of adsorption, diffusion, and desorption have, thus far, been limited to atoms and diatomic molecules, and in many instances adsorbate motion has been restricted to one dimension. Different theoretical approaches have been bjed to determine rate or diffusion coefficients. Thus, for example, dissociative adsorption of a diatomic molecule [42] has. been treated by solving the time-dependent Schrödinger equation $\psi(r, z ; t)=\psi(r, z ; 0) \exp [-i H t 2 \pi / h]$

where $\psi(r, z ; t)$ is the wave function for the adsorbate, $r$ is the atomatom bond distance, $z$ is the distance of the molecule's center of mass above the plane of the adsorption surface, and $\mathbf{H}$ is the Hamiltonian operator. Dissociative adsorption is assumed to occur 
when $r$ exceeds a prescribed critical value. By contrast, phononinduced desorption of atoms and molecules has been handled by determining the probability of finding an oscillator in the state $n$ at time $t, P_{n}(t)[43,44]$. The oscillators can occupy two types of states: bound states separated by discrete energy intervals or free states of continuous energy. The temporal evolution of $P_{n}(t)$ is governed by the master equation

$P_{n}(t)=P_{n}(0) \exp [-W t]$

where $W$ is the matrix of transition probabilities for bound-bound and bound-free transitions. The rate coefficient for desorption is then given by

$k_{d}^{(1)}=\left[\sum_{n, m}\left(W^{-1}\right)_{n m} P_{m}(0)\right]^{-1}$

Equation 2.94 has also been used to determine the hopping frequency for surface diffusion [45]. In such a case, the elements of $W$ are obtained using the potential function appropriate for diffusion. 
Table 2.1

Translational, rotational, and vibrational partition functions for molecules [37]

\begin{tabular}{|c|c|c|c|}
\hline Molecule & $\begin{array}{l}\text { Translation } \\
\mathrm{r}^{2} 2 \pi \mathrm{mkb} / \mathrm{h} 2\end{array}$ & $\begin{array}{l}\text { Rotation } \\
8 \pi^{2} k_{b} T I / h^{2} \sigma\end{array}$ & $\begin{array}{l}\text { Vibration } \\
1 /\left(1-\exp \left[-h v / k_{b} T\right]\right)\end{array}$ \\
\hline $\begin{array}{l}\mathrm{H}_{2} \\
\mathrm{CO} \\
\mathrm{Cl}_{2} \\
\mathrm{Br}_{2}\end{array}$ & $\begin{array}{r}33 \\
460 \\
1200 \\
2600\end{array}$ & $\begin{array}{r}2.9 \\
180 \\
710 \\
2100\end{array}$ & $\begin{array}{l}1.0 \\
1.0 \\
1.3 \\
1.7\end{array}$ \\
\hline
\end{tabular}

Area of a unit cell, $r 2=10.15 \mathrm{~cm}^{2} ; \mathrm{m}, l, v$, and $\sigma$ are the mass, moment of inertia, vibrational frequency, and symmetry number of a molecule; $T=500 \mathrm{~K}$.

Table 2.2

Range of the preexponential factor from absolute rate theory [37]

\begin{tabular}{|c|c|c|}
\hline Process & $\begin{array}{l}\text { Preexponential } \\
\text { factor }\end{array}$ & Units \\
\hline Molecular adsorption & $10 \cdot 10.1 \mathrm{j} \cdot 17$ & $\mathrm{~cm}^{3} \mathrm{~s} \cdot 1$ \\
\hline Dissociative adsorption & $10-10 \cdot: 0.17$ & $\mathrm{~cm}^{3} \mathrm{~s} \cdot 1$ \\
\hline Molecular desorption & 1013.1010 & s-1 \\
\hline Associative desorption & 10.4 .104 & $\mathrm{~cm}^{2} \mathrm{~s}^{-1}$ \\
\hline Langmuir-Hirishelwoood reaction & $10 \cdot 4.104$ & $\mathrm{~cm}^{2} \mathrm{~s}-1$ \\
\hline Eley-Rideal reaction & $10 \cdot 6 \cdot 10 \cdot 17$ & $\mathrm{~cm}^{3} \mathrm{~s}-1$ \\
\hline Unimolecular reaction & $1012-1013$ & s-1 \\
\hline Surface diffusion & $10.2 \cdot 10.4$ & $\mathrm{~cm}^{2} \mathrm{~s}-1$ \\
\hline
\end{tabular}



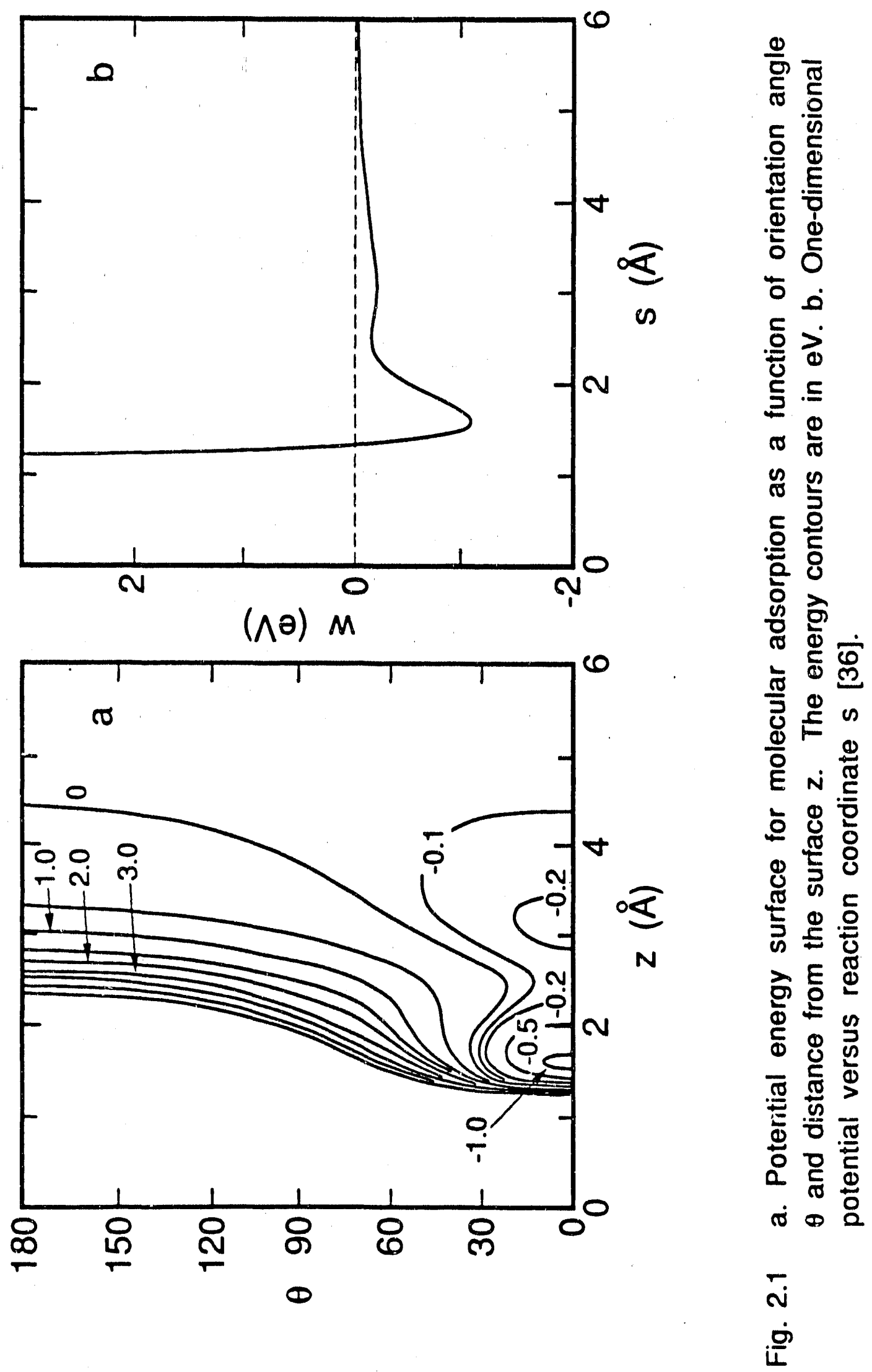

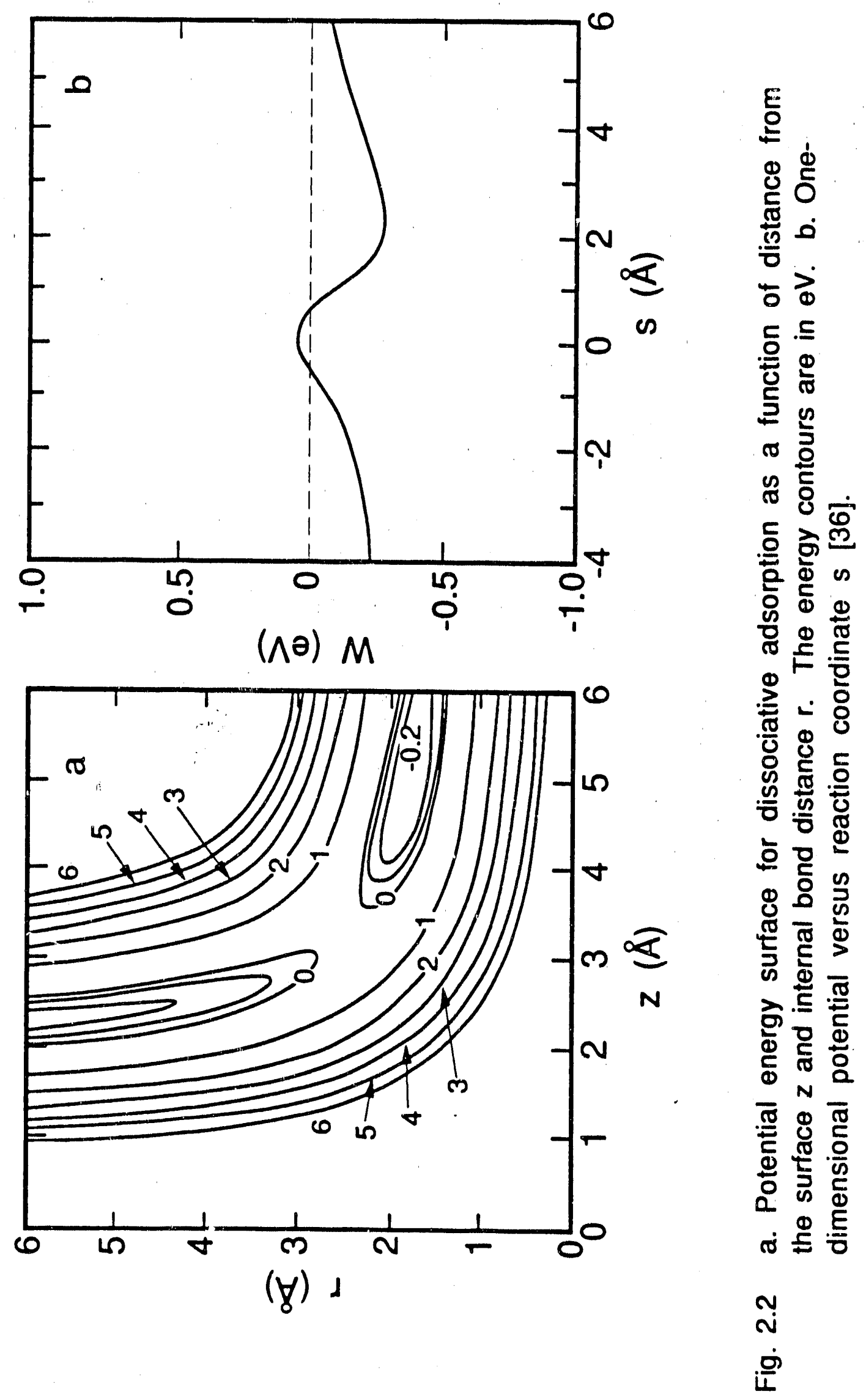

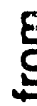

ญ

ن

ธे

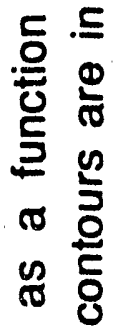

드 ㅎํ요

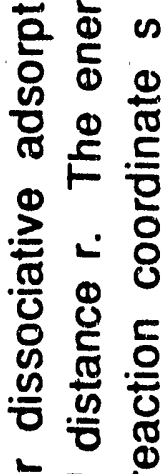

응 옹

洁

क․․ㄹ.

这 帘

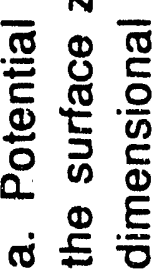

กั 


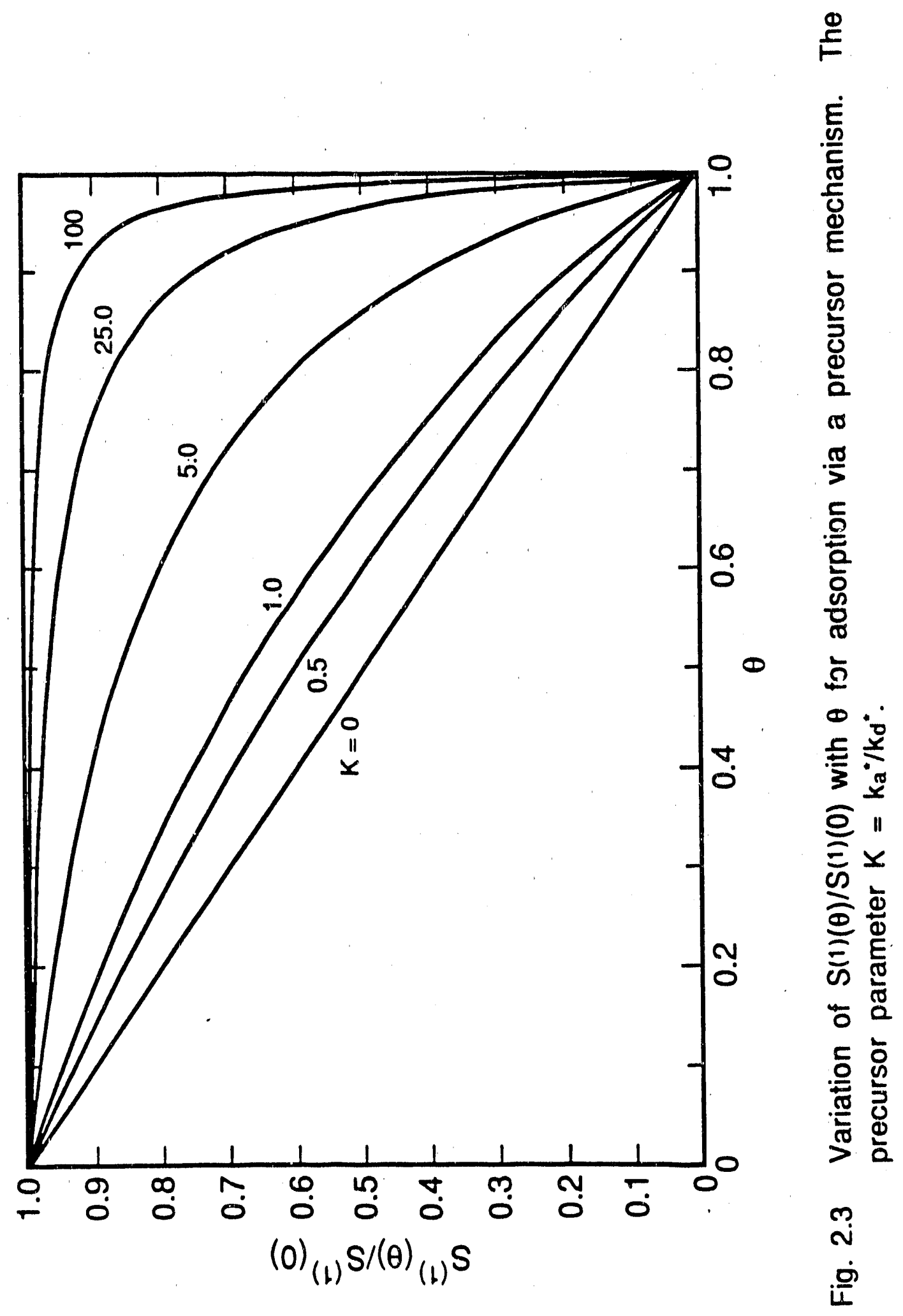



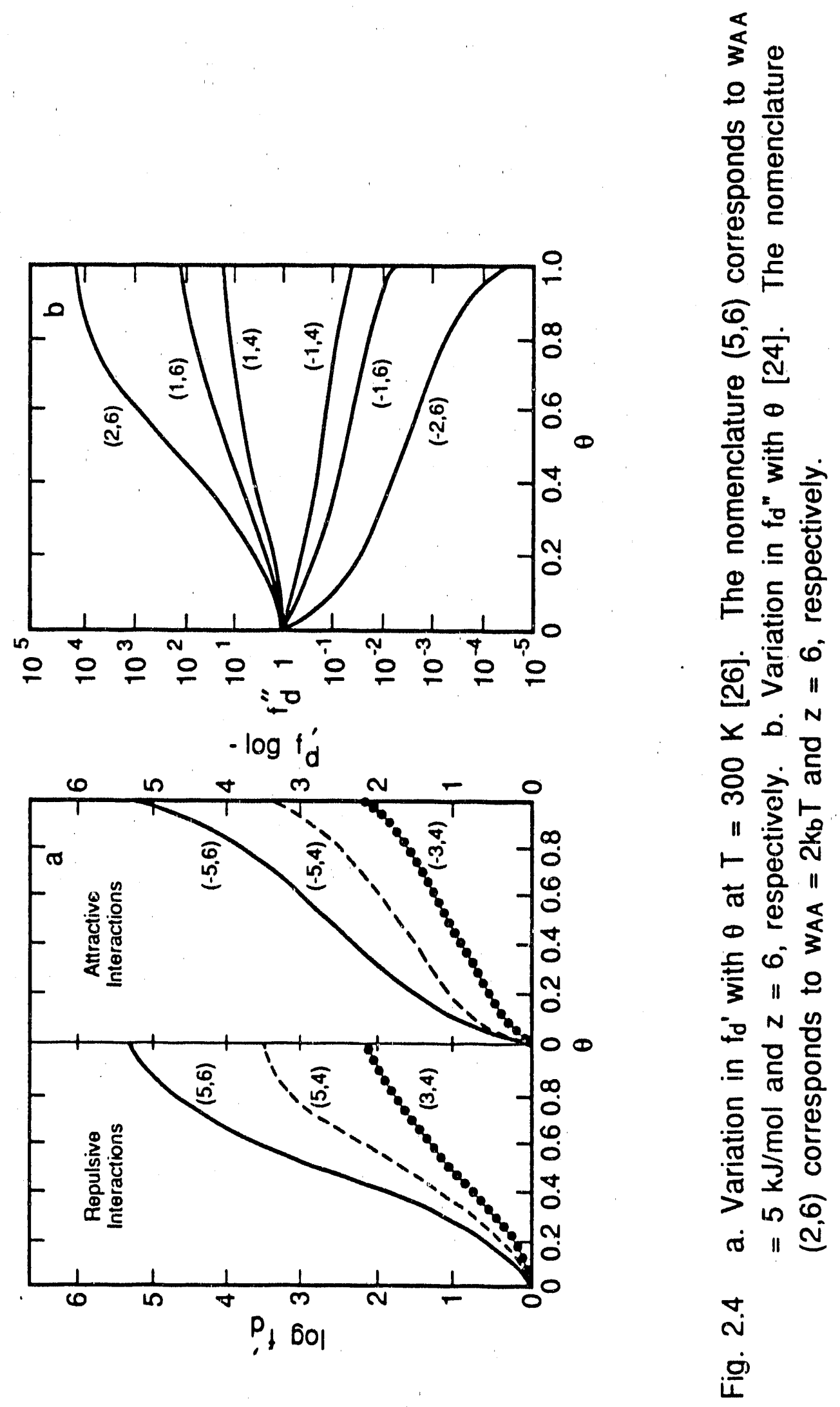


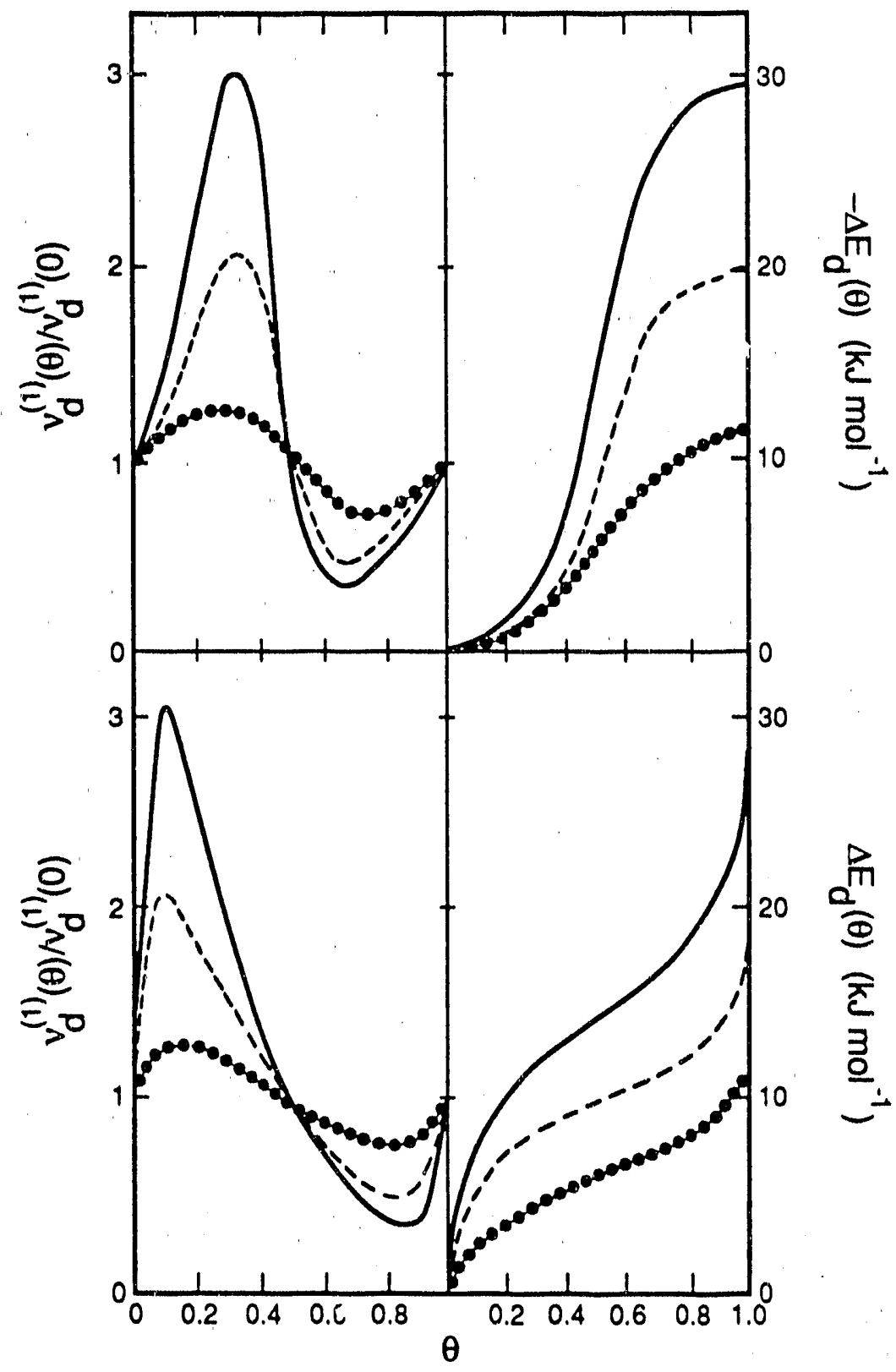

Fig. 2.5 Variation of $v_{d}(1)(\theta) / v_{d}(1)(0)$ and $\Delta E_{d}(\theta)$ with $\theta$ for repulsive (top panels) and attractive (bottom panels) interactions [26]. Solid line: $|\mathrm{WAA}|=5 \mathrm{~kJ} / \mathrm{mol}, z=6$; dashed line: $\mid$ WAA $\mid=5 \mathrm{~kJ} / \mathrm{mol}, z=4$; dotted line: $\mid$ WAA $\mid=$ $3 \mathrm{~kJ} / \mathrm{mol}, z=6$. 


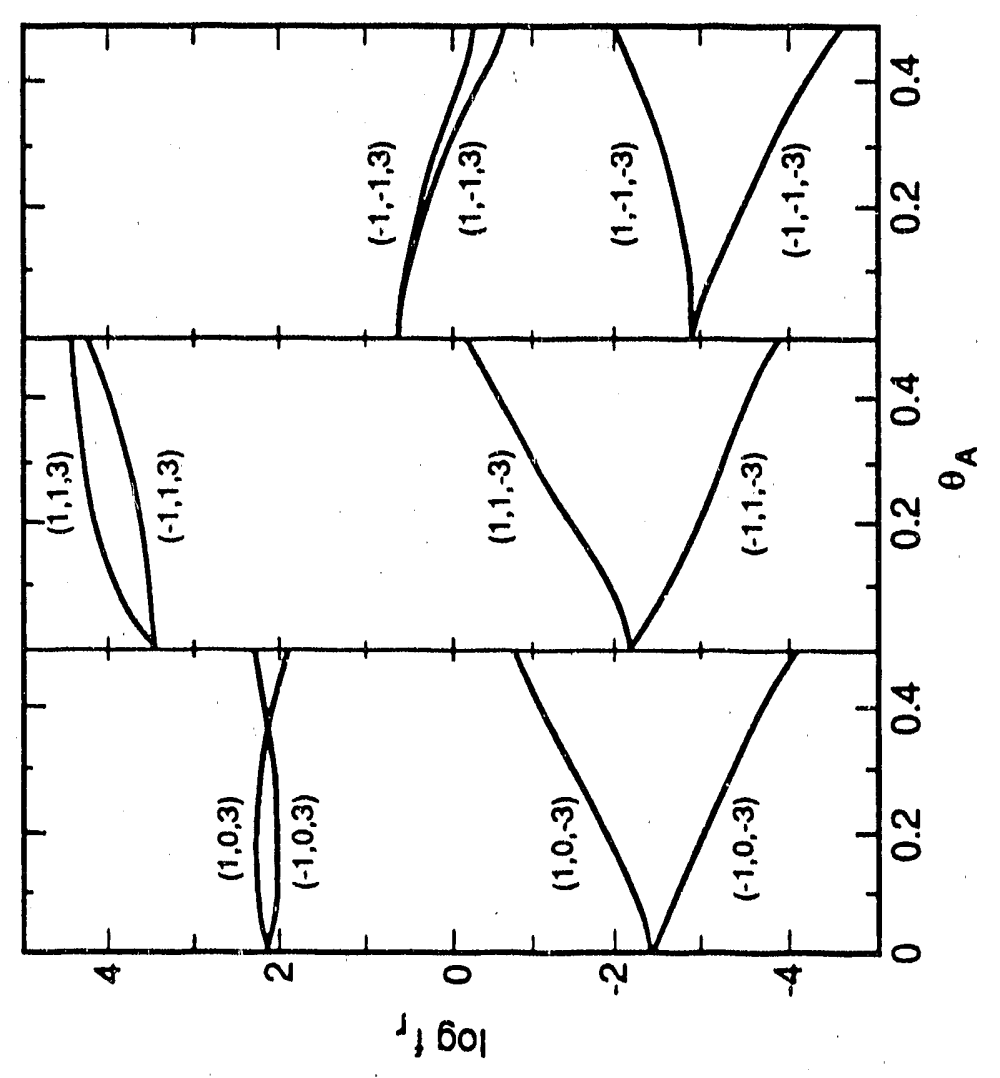

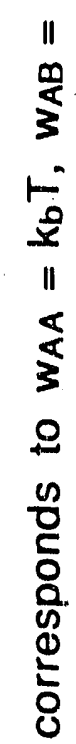

क

耑

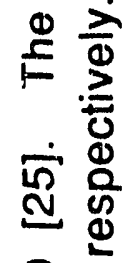

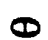

至寅

$\sim 11$

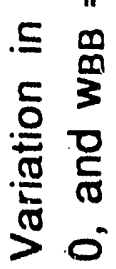

0
ำ
in 


\subsection{ADSORPTION}

\subsection{Molecular Dynamics}

Arumainayagam et al. [46] have used a Langevin model to calculate $S(1)(0)$ for $\mathrm{Xe}$ adsorption on $\mathrm{Pt}(111)$. Morse potentials were used to represent the Xe-surface interactions. During the course of a trajectory, adsorbate sticking was assumed to occur when the Xe-surface interactions fell below $-2 k_{b} T$. As seen in fig. 3.1, the level of agreement between the experimental and predicted values of $S(1)(0)$ is quite high. The authors noted that this level of agreement could only be obtained by adjusting the parameters of the Morse potential responsible for the steepness of the repulsive wall and the amount of surface corrugation. The observed reduction in $S(1)(0)$ with increasing adsorbate kinetic energy was attributed to inefficient energy transfer between the adsorbate and the surface.

Using an approach similar to that described above, Muhlhausen et al. [47] have determined $S(1)(0)$ for $N O$ adsorption on the (111) faces of $\mathrm{Pt}$ and $\mathrm{Ag}$. The NO-surface potential included a term to account for the orientation of NO relative to the surface. For both surfaces, $S(1)(0)$ was determined to be $\sim 0.7$ at $300 \mathrm{~K}$ and to decrease monotonically to $\sim 0.08$ at $2000 \mathrm{~K}$. The dependence of $S(1)(0)$ on the incident kinetic energy of NO was also examined and $S(1)(0)$ was observed to decrease with increasing kinetic energy of NO.

The sticking coefficient of $\mathrm{Si}$ on $\mathrm{Si}(100)$ has been examined by NoorBatcha et al. [48]. The adsorbate-surface potential was described by adatom-surface Morse potentials and a Keating 
potential which accounts for the bending and stretching modes of the lattice. The criterion for adsorbate sticking was an attractive interaction for a duration of more than five vibrational periods. The value of $S(1)(0)$ was determined to be 0.96 at $1500 \mathrm{~K}$ and to be relatively insensitive to temperature.

Molecular dynamics simulations of the dissociative adsorption of $\mathrm{H}_{2}$ on $\mathrm{Cu}(100)$ have been carried out by Gelb and Cardillo $[30,31]$. A LEPS potential energy surface was used to describe the interactions of $\mathrm{H}_{2}$ and $H$ with the metal surface. Dissociative adsorption was assumed to occur when the $\mathrm{H}_{2}$ internuclear separation distance exceeded $3.5 \AA$. As shown in fig. 3.2, their calculations predict that $S(2)(0)$ passes through a maximum with increasing kinetic energy of the $\mathrm{H}_{2}$ molecule. The increase in $\mathrm{S}(2)(0)$ observed at low kinetic energies is attributed to an increase in the probability of surmounting the activation barrier for dissociative adsorption. For very large values of the kinetic energy, however, the time of interaction of the adsorbate with the surface is so small as to preclude sufficient rearrangement of the $\mathrm{H}_{2}$ molecules into configurations favorable for dissociation, and hence, the value of $S(2)(0)$ decreases as the kinetic energy increases.

Lee and DePristo [33-35] have reported on the dissociation kinetics of $\mathrm{H}_{2}$ on $\mathrm{Ni}$ and $\mathrm{Cu}$ crystal faces. A generalized Langevin model was used to simulate adsorbate trajectories and a LEPS potential energy surface was used to describe the $\mathrm{H}_{2}$ - and $\mathrm{H}$-metal interactions. Dissociative adsorption was assumed to occur when the $\mathrm{H}_{2}$ internuclear separation distance exceeded $2.8 \AA$. A comparison of the predicted and experimental values of $S(2)(0)$ as a 
function of incident kinetic energy of the adsorbate is given in tables 3.1 and 3.2. For the Cu surfaces, both the predicted values of $S(2)(0)$ and the dependence of these values on kinetic energy are in fair agreement with the experimental observation [49,50], whereas for the $\mathrm{Ni}$ surfaces, the predicted values of $\mathrm{S}(2)(0)$ and the dependence on kinetic energy do not agree well with the experimental data [51,52]. Using the same approach, Kara and DePristo [53] have calculated $S(2)(0)$ for $\mathrm{N}_{2}$ adsorption on $W(110)$. As can be seen in fig. 3.3, the agreement between theory and experiment [54] is very good in this case.

\subsection{Transition-State Theory}

The influence of precursor states on molecular adsorption has been examined by Doren and Tully [36] using dynamic TST. In their model, adsorbate-surface interactions were described by a onedimensional potential of mean force $W(z)$, which depends only on $z$, the distance of the molecular center of mass from the surface. The presence of a precursor state was defined by the occurrence of a secondary minimum in $W(z)$, located between the chemisorbed state and $z$ approaching infinity. As illustrated in $3.4 \mathrm{a}$ for the case of $\mathrm{CO}$ adsorption on $\mathrm{Ni}(111)$, the depth of the well for the precursor state decreases with increasing temperature, whereas the height of the activation barrier for dissociative adsorption increases. Figure $3.4 \mathrm{~b}$ shows that the sticking coefficient for molecular adsorption of $\mathrm{CO}$ first decreases and then gradually increases with increasing temperature. This behavior can be explained as follows. At low 
temperatures, the kinetic energy of the impinging adsorbate is small and consequently very little energy transfer is required for the adsorbate to be trapped into the precursor state. Since the barrier for subsequent trapping into the adsorbate state is small at low temperatures, adsorption into that state proceeds rapidly and hence $S(1)(0)$ is large. At intermediate temperatures, the activation energy barrier becomes more pronounced (see fig. 3.4a), and the inability of adsorbates to surmount this higher barrier leads to lower values of the sticking coefficient. At high temperatures, the kinetic energy of the incident molecules is now sufficient to directly overcome the activation energy barrier without initial trapping into the precursor state. The behavior observed at low and high temperatures is referred to in the literature as precursor-mediated adsorption and direct adsorption, respectively.

Truong et al. [55] have used a variational TST approach which included quantum effects to determine the activation energy for dissociative adsorption, Ediss, of $\mathrm{H}_{2}$ and $\mathrm{D}_{2}$ on $\mathrm{Ni}(100)$, (111), and (110) surfaces. The adsorbate-surface interactions were described by a LEPS potential energy surface. To account for the quantum effects of tunneling and reflection, KTST was multiplied by a groundstate transmission coefficient. The apparent activation energies are listed in table 3.3. As can be seen, the value of $E_{d i s s}$ depends on the crystal face, the temperature, and the molecular weight of the adsorbate. For $\mathrm{H}_{2}$ adsorption on $\mathrm{Ni}(100)$, Ediss is negative up to 500 $K$, indicating that the classical barrier to desorption lies below the reactant energy. On $\mathrm{Ni}(110)$, the value of $E_{\text {diss }}$ is less than or equal to $0.4 \mathrm{kcal} / \mathrm{mol}$ below $500 \mathrm{~K}$, whereas on $\mathrm{Ni}(111)$, it is about 1.0 
$\mathrm{kcal} / \mathrm{mol}$ below $500 \mathrm{~K}$. The complex dependence of Ediss with temperature for both $\mathrm{H}_{2}$ and $\mathrm{D}_{2}$ was partially attributed to the effects of tunneling.

Adams and Doll [56] have also used a dynamic TST model to examine the influence of precursor states on the sticking coefficient for dissociatively adsorbed species. Their calculations suggest that $S(2)(0)$ decreases with increasing temperature and is sensitive to the well depth in the precursor state and the rate of energy dissipation in the adsorbed state.

\subsection{Quantum Mechanical Models}

A quantum mechanical description of $\mathrm{H}_{2}$ adsorption on $\mathrm{Ni}(100)$ has been developed by Jackson and Metiu [42]. The interactions of $\mathrm{H}_{2}$ and $H$ with the metal surface were described by a LEPS potential energy surface. The principal features of the energy surface were the barriers for molecular adsorption, $E_{a}$, dissociation, Ediss, and atomic diffusion, Ediff. The adsorption behavior was found to depend on the relative magnitudes of $E_{a}$ and $E_{d i s s . ~ W h e n ~} E_{a}=0$ and $E_{d i s s}<E_{\text {diff }}$ (restricted adsorbate mobility), the dissociation probability $S(2)(0)$ was larger than when $E_{\mathrm{a}}=0$ and $E_{\text {diss }}>E_{\text {diff }}$ (mobile adsorbate). This behavior is attributed to the fact that restriction of adsorbate mobility increases the probability for $\mathrm{H}$-atom recombination and hence, lowers the value of $S(2)(0)$. As can be seen in fig. $3.5, S(2)(0)$ increases with increasing kinetic energy and is smaller for the larger values of Ediss. The quantum nature of the dissociation process is especially evident for $E_{\text {diss }}=-4.423 \mathrm{eV}$. Classically, 
dissociation should occur with unit probability for all kinetic energies, since Ediss is below the zero-point energy of $\mathrm{H}_{2}$. The process of quantum reflection upon traversing a potential change, however, leads to much lower values of $S(2)(0)$.

Using an approach similar to the one described above, Chiang and Jackson [32] have investigated the isotope effect for adsorption of $\mathrm{H}_{2}, \mathrm{D}_{2}$ and $\mathrm{T}_{2}$ on $\mathrm{Ni}(100)$. Their calculations showed that for a fixed value of the incident kinetic energy, $S(2)(0)$ decreases with increasing molecular weight. This behavior arises from the influence of the zero-point energy on the apparent barrier to dissociation. In qualitative agreement with this prediction, Hamza and Madix [50] have observed larger values of $\mathrm{S}(2)(0)$ for $\mathrm{H}_{2}$ dissociation on $\mathrm{Ni}(100)$ than for $D_{2}$.

Asscher et al. [57] have calculated $\mathrm{S}(2)(0)$ for $\mathrm{N}_{2}$ on $\operatorname{Re}(0001)$ and $\mathrm{Fe}(111)$. The principal feature of the potential energy surface was an activation energy barrier to dissociation $E_{\text {diss }}=73 \mathrm{~kJ} / \mathrm{mol}$. It was shown that $S(2)(0)$ increases exponentially with increasing incident kinetic energy, in agreement with the experimental results for $\mathrm{N}_{2}$ on $\mathrm{Fe}(111)$ [58]. The low values of $\mathrm{S}(2)(0)$ observed for low initial kinetic energies were attributed to the large activation energy for dissociation. It is interesting to note that classically, non-zero values of $S(2)(0)$ would not have been expected at low kinetic energies for such a large value of $E_{d i s s ;}$ the observation of a finite value of $S(2)(0)$ is thus attributable to tunneling of the molecular wave function through the barrier. 


\subsection{Effects of Lateral Interactions}

King and Wells [59] have developed a lattice-gas model to treat the influence of lateral interactions on the rate of dissociative adsorption. For adsorption to occur, it was assumed that a pair of unoccupied nearest-neighbor sites must exist on the surface. The sticking coefficient for adsorption is thus given as $S(2)(\theta)=$ $S\left({ }^{2}\right)(0)$ PVV where PVV is given by eq. 2.41. A plot of $S(2)(\theta) / S(2)(0)$ is shown in fig. 3.6 as a function of the parameter $\eta / 2=[1-\exp (-$ $\left.W A A / k_{b} T\right)$ ]. At low coverages, $S(2)(\theta) / S(2)(0)$ is practically independent of $\eta_{1} / 2$, whereas at high coverage, $S(2)(\theta) / S(2)(0$ ) decreases more rapidly with increasing $\eta / 2$. The values of WAA for $\eta / 2$ evaluated at $T=300 \mathrm{~K}$ are also shown in fig. 3.6 and it can be seen that $S(2)(\theta) / S(2)(0)$ is not a strong function of WAA. It is also interesting to note some of the limiting forms of $S(2)(\theta) / S(2)(0)$. When WAA $=0, S(2)(\theta) / S(2)(0)=(1-\theta)^{2}$ which is equivalent to the expression for non-activated adsorption for randomly distributed adsorbates. For large repulsive interactions $\left(W_{A A} \rightarrow \infty\right)$, $S(2)(\theta) / S(2)(0)=1-2 \theta$ for $\theta \leq 0.5$, which represents pseudo first-order dissociative adsorption kinetics.

Zhdanov [60] has examined the influence of lateral interactions on the adsorption rate using a lattice-gas model. The sticking coefficient for adsorption was determined from absolute rate theory. For non-dissociative adsorption, the rate was given by

$$
r_{a}=\frac{k_{b} T}{h} \sum_{n} \frac{q_{A}^{\dagger}}{q_{A}} P_{V, n} \exp \left[\frac{-E_{a}^{0}}{k_{b} T}\right](1-\theta) N_{A, g}
$$


and the ratio of $S(1)(\theta) / S(i)(0)$ by

$$
\frac{S^{(1)}(\theta)}{S^{(1)}(0)}=\sum_{n} \frac{q_{A}^{\dagger}}{q_{A}} P_{V, n}(1-\theta)
$$

Figure 3.7 shows curves of $S(1)(\theta) / S(1)(0)$ for attractive, repulsive and no interactions, when $q_{A} t / q_{A}=0.3 n$. For a fixed value of $\theta$, $S(1)(\theta) / S(1)(0)$ is largest for attractive interactions. $S(1)(\theta) / S(1)(0)$ is also seen to decrease non-linearly with coverage, independent of the sign of WAA.

The influence of lateral interactions on adsorption kinetics has been investigated for the case of dissociative adsorption via a precursor mechanism. King and Wells [59] have shown that when intrinsic and extrinsic precursor states exist, $S(2)(\theta) / S(2)(0)$ can be written as

$\frac{S^{(2)}(\theta)}{S^{(2)}(0)}=\frac{1}{1+L\left(\frac{1}{P_{W}}-1\right)}$

where $\mathrm{L}_{\mathrm{C}} \mathrm{rd}^{*} /\left(\mathrm{r}_{\mathrm{a}}{ }^{*}+\mathrm{rd}^{*}\right)$. The desorption rates from extrinsic and intrinsic precursor states are denoted by $\mathrm{rd}^{*}$ and $\mathrm{rd}^{\circ}$, respectively, and the adsorption rate from the intrinsic precursor state is denoted by $\mathrm{ra}{ }^{*}$. If $\mathrm{ra}{ }^{*} \gg>\mathrm{rd}^{\circ}, \mathrm{rd}{ }^{*}$, then $\mathrm{L} \rightarrow 0$, whereas when $\mathrm{ra}{ }^{*} \ll \mathrm{rd}^{*}, \mathrm{rd}{ }^{*}$, then $L \rightarrow 1 . L$ is thus a measure of the relative importance of the adsorption and desorption processes. A plot of $S(2)(\theta) / S(2)(0)$ parametric in $L$ for a fixed value of $\eta / 2$ is shown in fig. 3.8a. When $L \rightarrow 0, S(2)(\theta) / S(2)(0)$ is larger than when $L \rightarrow 1$. Figure $3.8 b$ show a plot of $S(2)(\theta) / S(2)(0)$ parametric in $\eta / 2$ for a fixed value of $L$. At 
high coverage, $S(2)(\theta) / S(2)(0)$ is strongly dependent on the magnitude of WAA and decreases with increasingly repulsive values of WAA. It is significant to note that the shape and magnitude of the plots of $S(2)(\theta) / S(2)(0)$ differ considerably depending on whether precursor states do (see figs. 3.8a and 3.8b) or do not exist (see fig. 3.6).

King and Wells [59] have used eq. 3.3 to describe the dissociative adsorption of $N_{2}$ on a $W(100)$ surface. Values of the parameters required to fit eq. 3.3 to the experimental data are listed in table 3.4, and a comparison of the fitted and experimental results is shown in fig. 3.9. Good agreement is observed at both low and high temperatures. It is interesting to note that $L$ increases with increasing temperature, a trend which reflects the temperature dependence of the desorption rate.

The adsorption of molecular $\mathrm{N}_{2}$ on $\mathrm{Ru}(001)$ has been simulated by Hood et al. [61] using a Monte Carlo model. Adsorption was assumed to proceed via a precursor mechanism in which both intrinsic and extrinsic precursor sites could be occupied. Repulsive nearest-neighbor interactions between precursor and chemisorbed species were taken to be $0.25 \mathrm{kcal} / \mathrm{mol}$ and attractive next-nearest neighbor interactions were taken to be $0.45 \mathrm{kcal} / \mathrm{mol}$. As seen in fig. 3.10, the predicted variation in $S(1)(\theta)$ is in fair agreement with the experimentally observed trend $[62,63]$. The initial rise in $S(1)(\theta)$ is attributed to the formation of energetically favorable islands in which molecules are arranged in $\sqrt{3} \times \sqrt{3} \mathrm{R} 30^{\circ}$ domains, whereas the decrease in $S(1)(\theta)$ at high coverage is attributed to crowding effects. 
Table 3.1

Dissociative sticking probability of hydrogen on $\mathrm{Cu}$ surfaces for $T_{s}=1000 \mathrm{~K}$ [33]

\begin{tabular}{lcccc}
\hline $\begin{array}{l}\text { Kinetic } \\
\text { Energy(eV) }\end{array}$ & Calc. & Exp.a & Calc. & Exp.a \\
\hline 0.15 & 0.03 & 0.08 & $<0.01$ & 0.03 \\
0.20 & 0.06 & 0.115 & 0.01 & 0.045 \\
0.30 & 0.11 & 0.135 & 0.06 & 0.095 \\
0.40 & 0.15 & 0.14 & 0.10 & 0.100 \\
0.60 & 0.27 & - & 0.27 & - \\
\hline
\end{tabular}

aFrom Refs. [49,50].

Table 3.2

Dissociative sticking probability of hydrogen on $\mathrm{Ni}$ surfaces for $\mathrm{T}_{s}=300 \mathrm{~K}$ [33]

\begin{tabular}{|c|c|c|c|c|c|c|}
\hline \multirow{2}{*}{$\begin{array}{l}\text { Kinetic } \\
\text { Energy }(\mathrm{eV})\end{array}$} & \multicolumn{2}{|c|}{$\mathrm{Ni}(100)$} & \multicolumn{2}{|c|}{$\mathrm{Ni}(110)$} & \multicolumn{2}{|c|}{$\mathrm{Ni}(111)$} \\
\hline & Calc. & Exp.a & Calc. & Exp.b & Calc. & Exp.b \\
\hline 0.03 & 0.17 & 0.4 & 0.55 & 0.96 & 0.09 & 0.02 \\
\hline 0.05 & 0.18 & 0.6 & 0.52 & 0.96 & 0.08 & 0.07 \\
\hline 0.07 & 0.18 & 0.7 & 0.52 & 0.96 & 0.09 & 0.10 \\
\hline 0.10 & 0.20 & 0.8 & 0.52 & - & 0.09 & 0.27 \\
\hline 0.14 & 0.20 & - & 0.52 & - & 0.10 & - \\
\hline 0.20 & 0.20 & - & 0.50 & - & 0.12 & - \\
\hline
\end{tabular}

aFrom Refs. [51].

bFrom Refs. [52]. 
Table 3.3

Activation energy (kcal/mol) for dissociative adsorption of hydrogen and deuterium on $\mathrm{Ni}$ surfaces [55]

\begin{tabular}{crrrrrr}
\hline$T(\mathrm{~K})$ & \multicolumn{2}{c}{$\mathrm{Ni(100)}$} & \multicolumn{2}{c}{$\mathrm{Ni(110)}$} & \multicolumn{2}{c}{$\mathrm{Ni(111)}$} \\
& $\mathrm{H}_{2}$ & $\mathrm{D}_{2}$ & $\mathrm{H}_{2}$ & $\mathrm{D}_{2}$ & $\mathrm{H}_{2}$ & $\mathrm{D}_{2}$ \\
& & & & & & \\
\hline $100-140$ & -0.87 & -1.31 & 0.29 & 0.10 & 0.99 & 0.61 \\
$140-200$ & -1.23 & -1.02 & 0.35 & 0.09 & 0.92 & 0.63 \\
$200-300$ & -1.01 & -0.67 & 0.40 & 0.05 & 0.86 & 0.70 \\
$300-500$ & -0.71 & -0.30 & 0.37 & -0.05 & 0.94 & 1.11 \\
$500-800$ & 0.16 & 0.54 & -0.28 & -0.29 & 1.59 & 2.21 \\
\hline
\end{tabular}

Table 3.4

Values of the parameters used to represent the data in fig. 3.9 [59]

\begin{tabular}{lrlll}
\hline$T_{s}(\mathrm{~K})$ & $N_{s}(1014 \mathrm{~cm}-2)$ & $L$ & $\eta / 2$ & $S(2)(0)$ \\
\hline 300 & 9.5 & 0.082 & 0.989 & 0.585 \\
433 & 10.0 & 0.157 & 0.987 & 0.49 \\
663 & 10.5 & 0.256 & 0.977 & 0.31 \\
773 & 9.5 & 0.517 & 0.986 & 0.21 \\
\hline
\end{tabular}


56

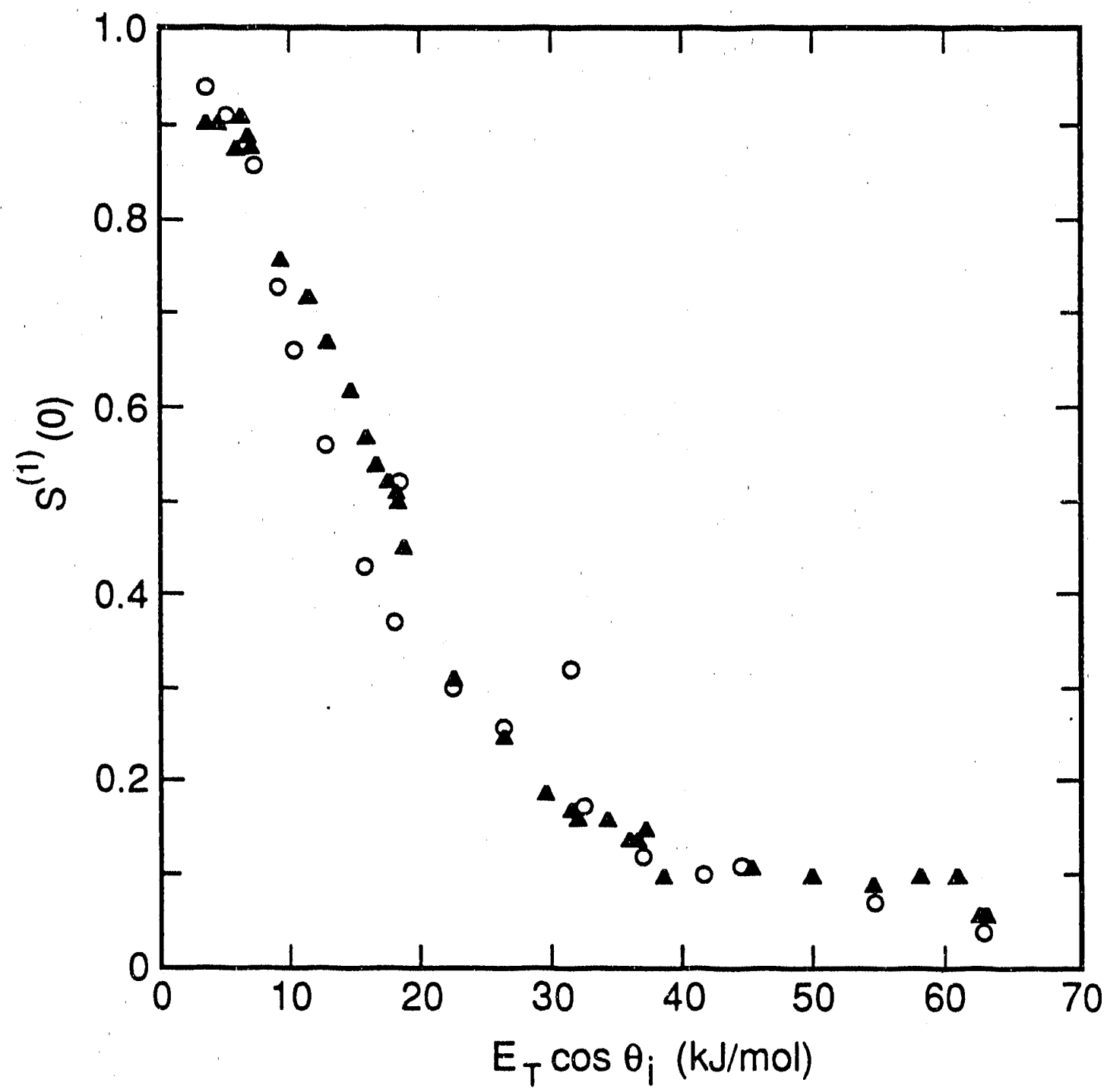

Fig. 3.1 Simulated (O) and experimental ( $\mathbf{\Delta})$ variation of $S(1)(0)$ versus $E_{T} \cos \theta_{i}$ for $X e$ adsorption on $P t(111)$ [46]. ET is the incident kinetic energy and $\theta_{i}$ is the incident angle measured from the surface normal. 


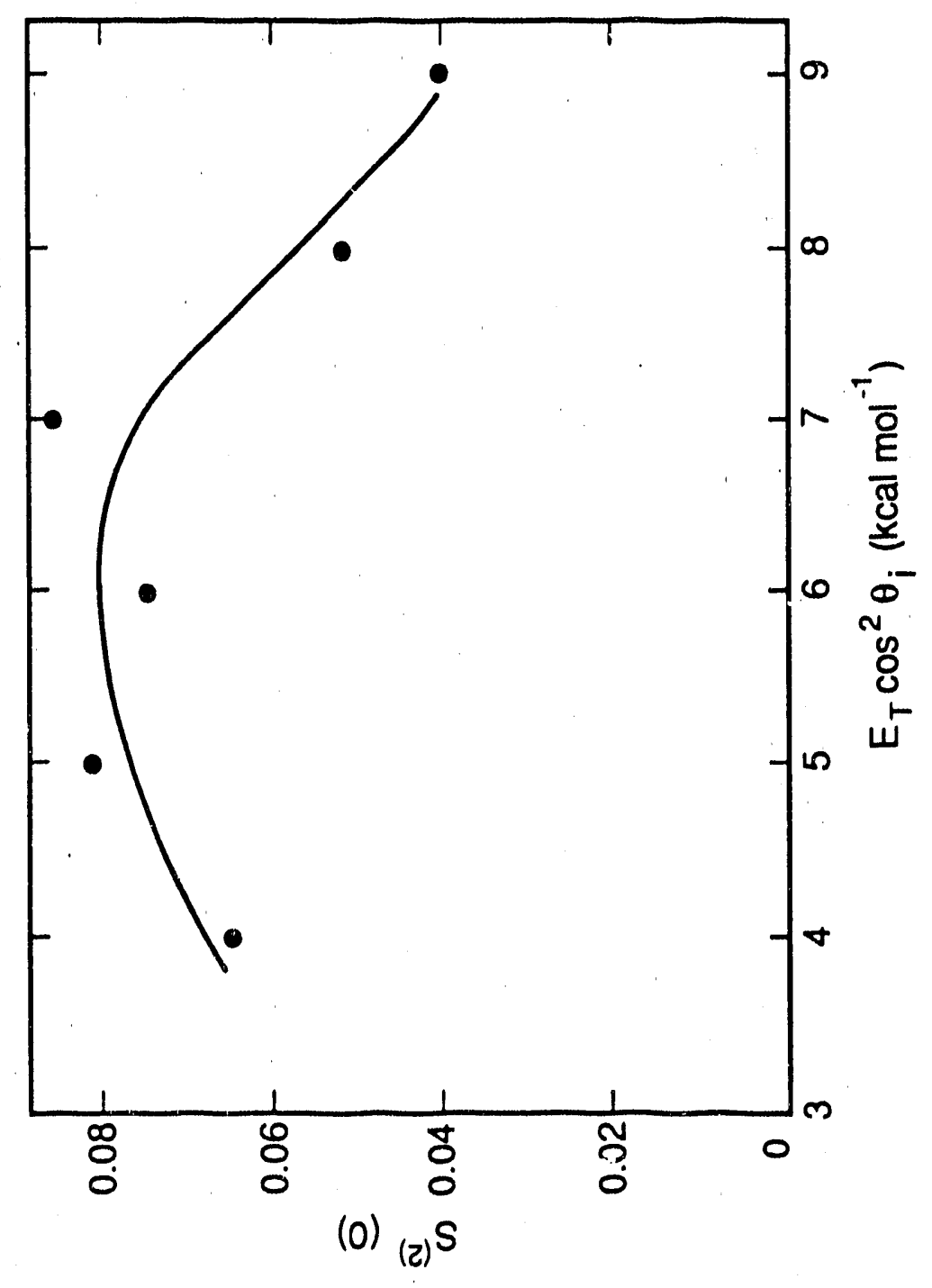

$\dot{\pi}$

용

ปั

등 잉

든 는

은 뽕

o $E$

ชั

N

은 등

ธ $\frac{0}{0}$

ชै. 오

넹ㅇㅇ

는

先

$\sum^{0}$ 唨

응 근모

जั

ั0 으

등 원

을 돋

它 EF

-

응 응

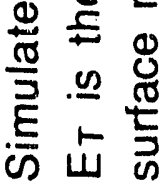

กั

iำ 


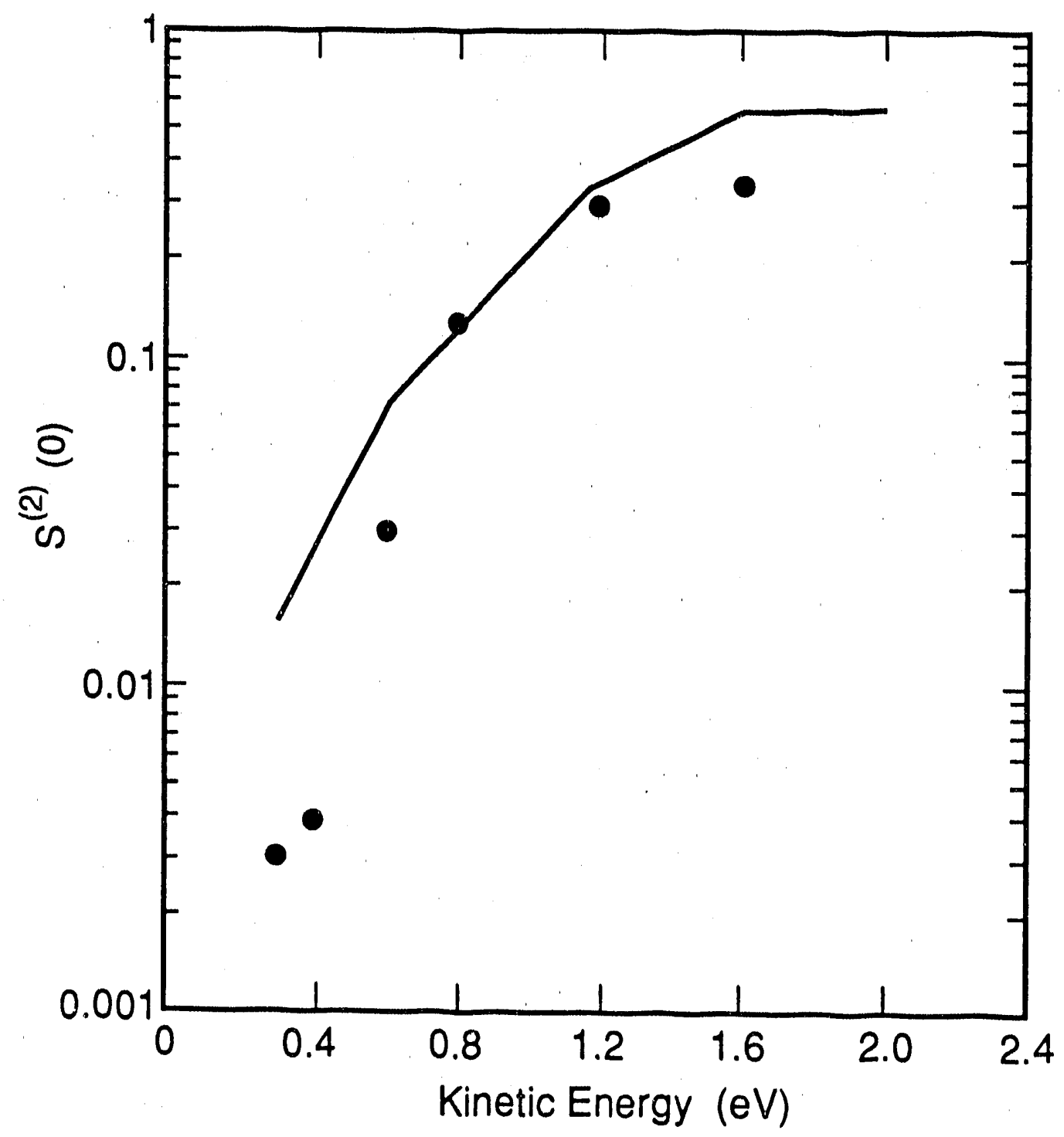

Fig. 3.3 Simulated (solid line) [53] and experimental (circles) [54] variation of $S(2)(0)$ versus incident kinetic energy for $\mathrm{N}_{2}$ adsorption on $\mathrm{W}_{(110)}$. 


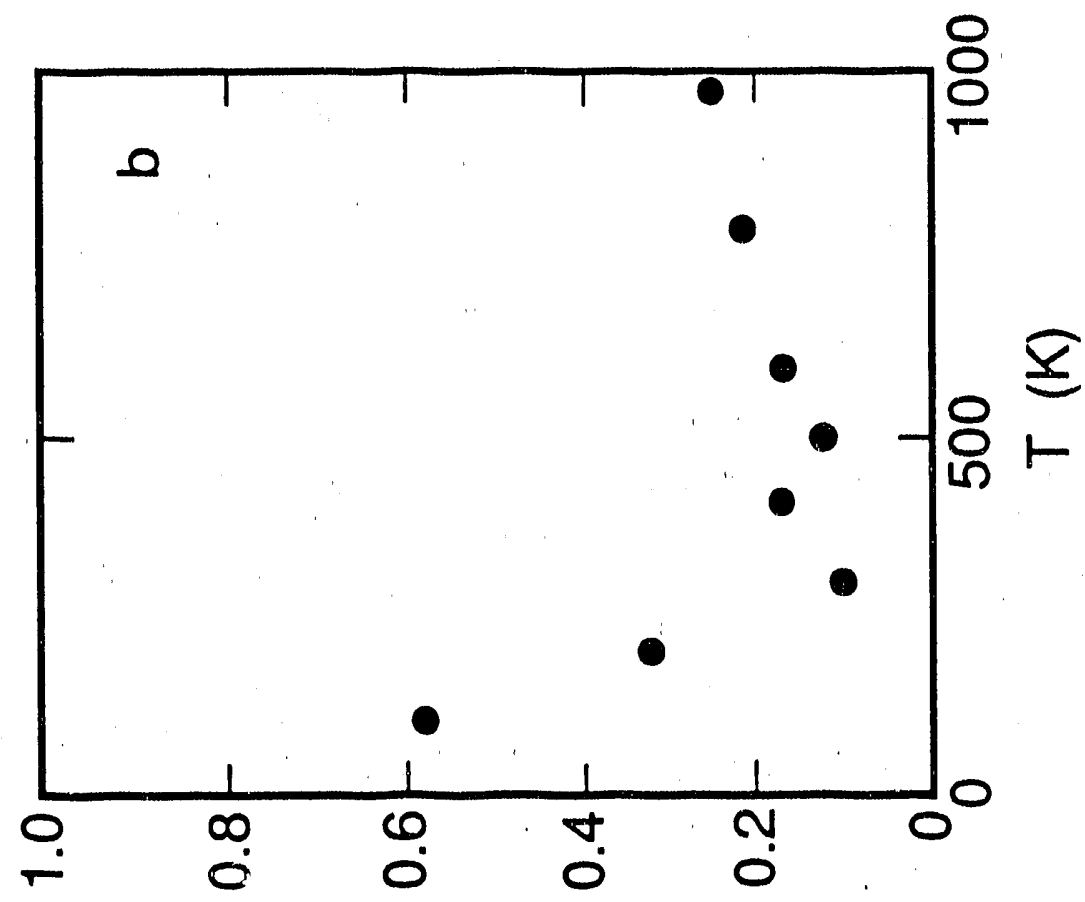

(0) ${ }_{(1)} \mathrm{S}$

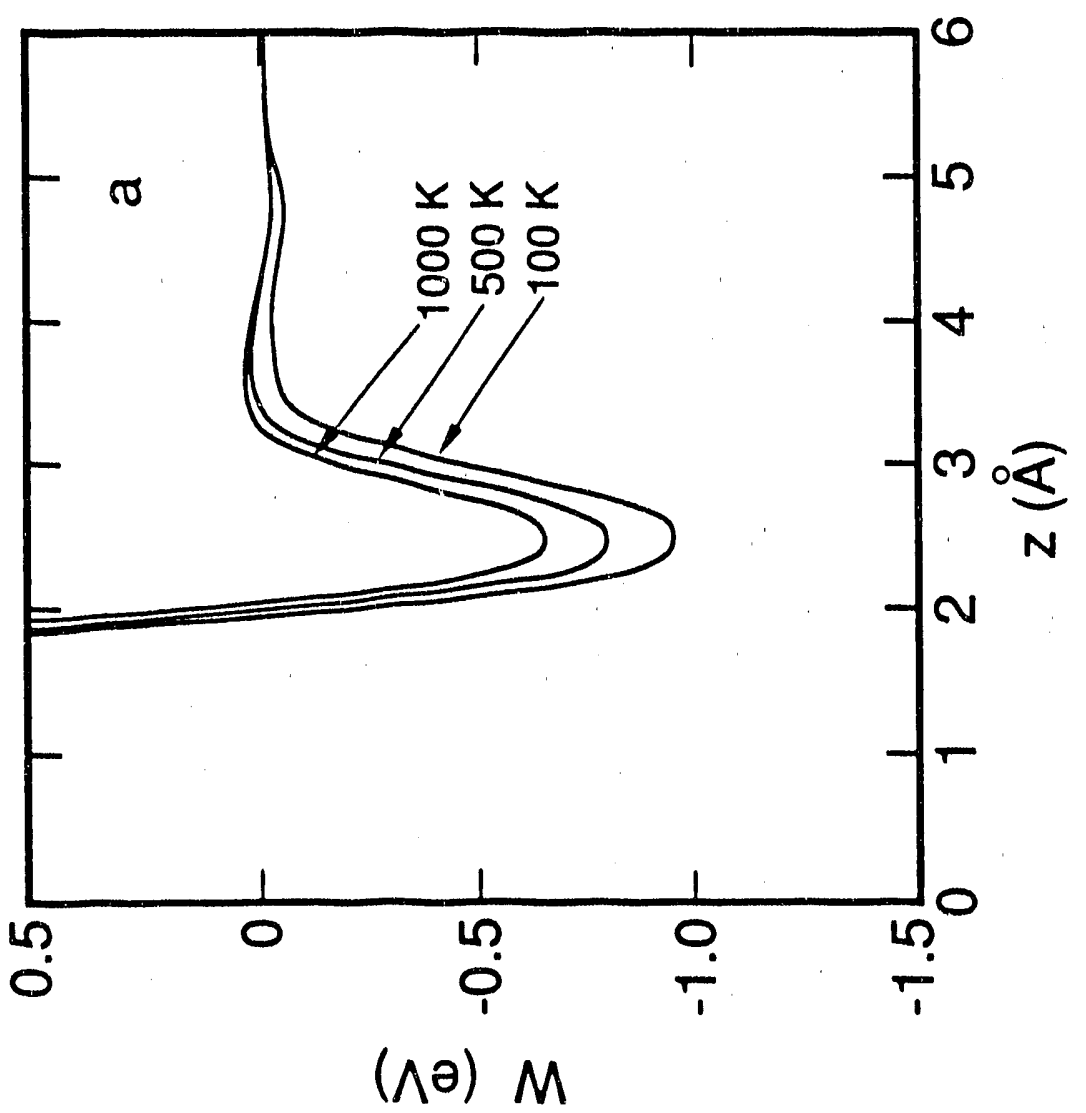

م

$\dot{F}$

क्ष

之远

드

O

홍

응

\%

就

๑

$\infty O$

ว

운

䆑卜

竞

乌

\$응

ญ

$\div$

등

ฐ

ธั。

$\bar{\nabla}$

일

Фั

응

ते

ले

iํํำ 


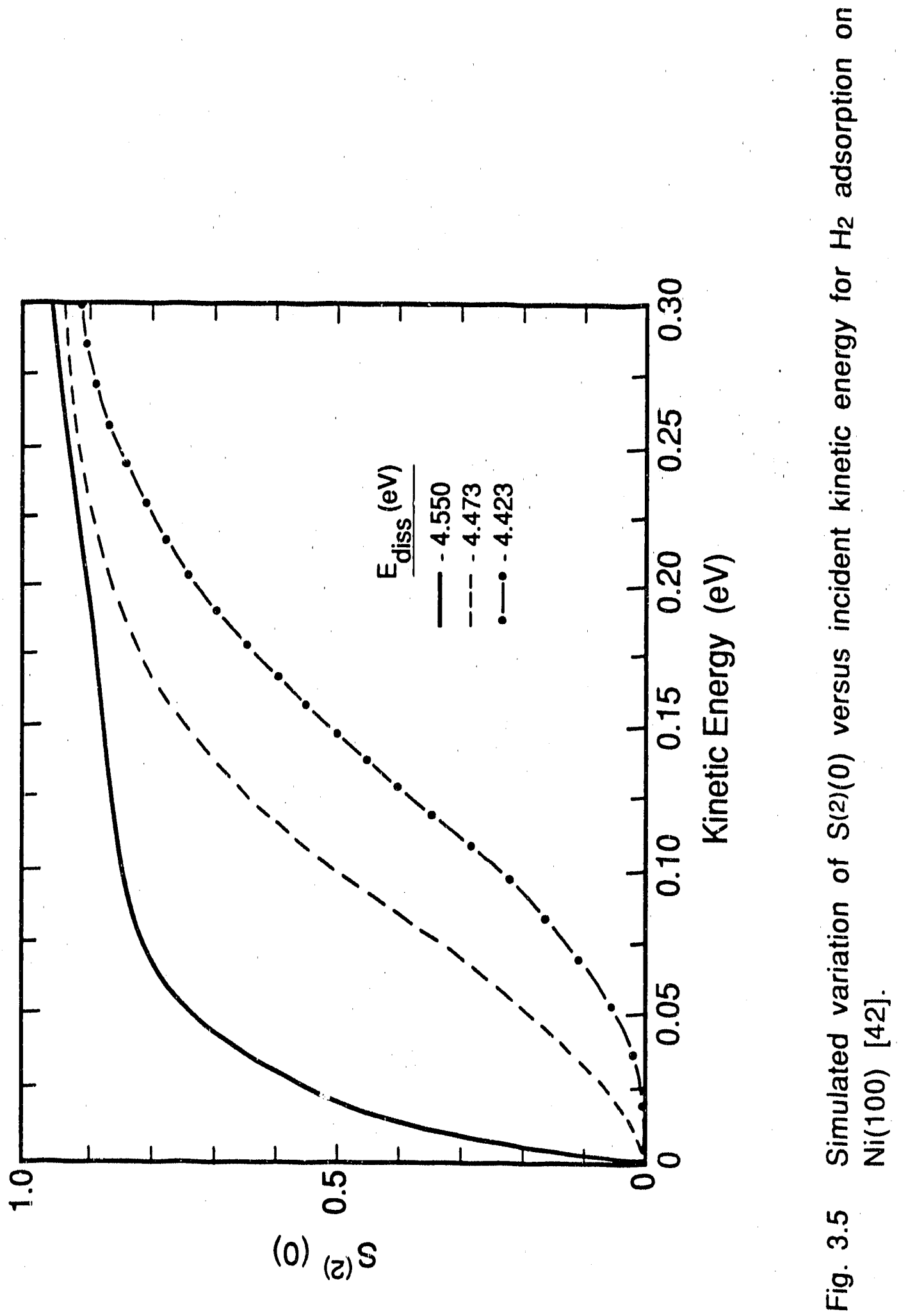




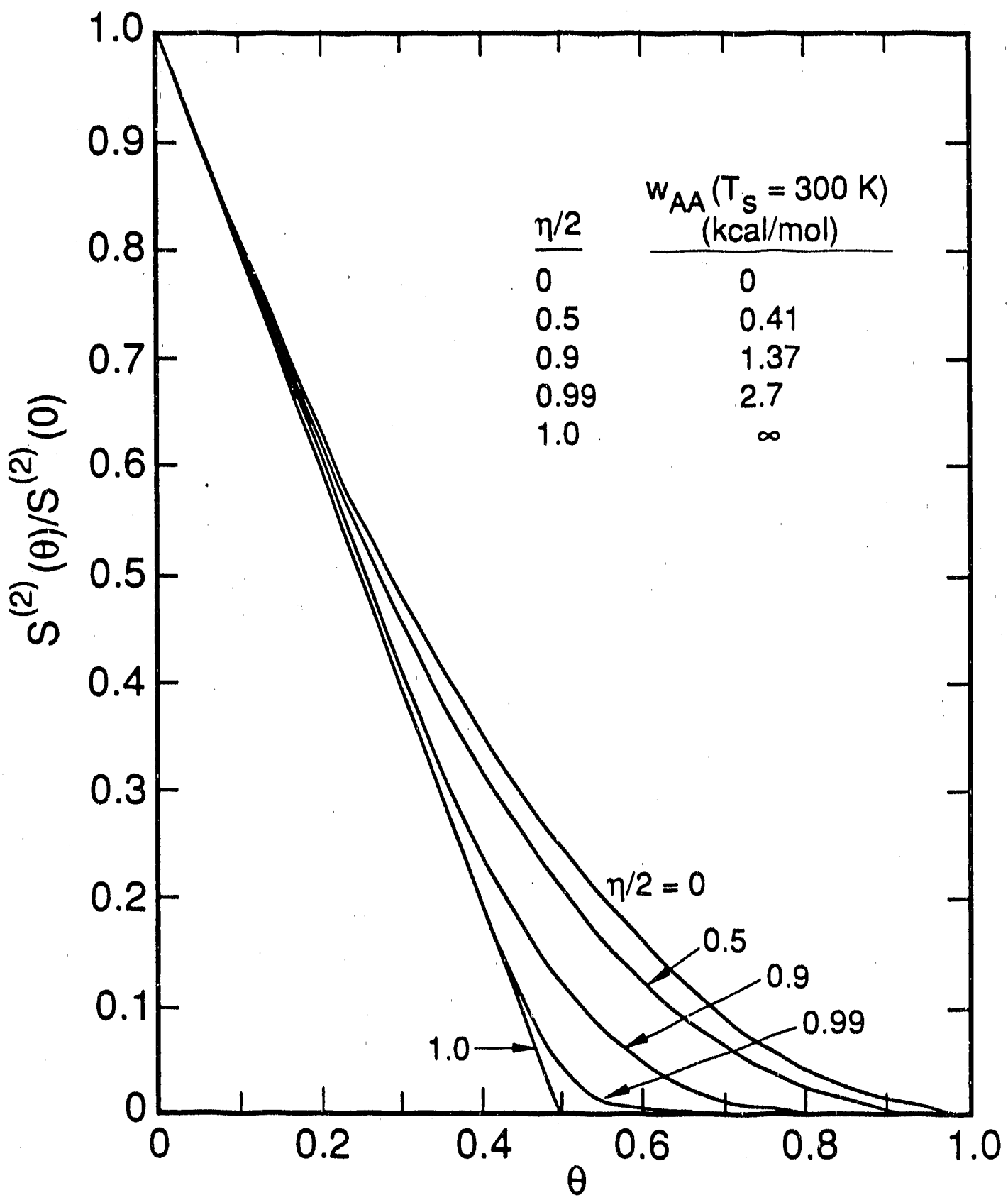

Fig. 3.6 Variation in $S(2)(\theta) / S(2)(0)$ versus $\theta$ for repulsive nearestneighbor interactions [59]. 
62

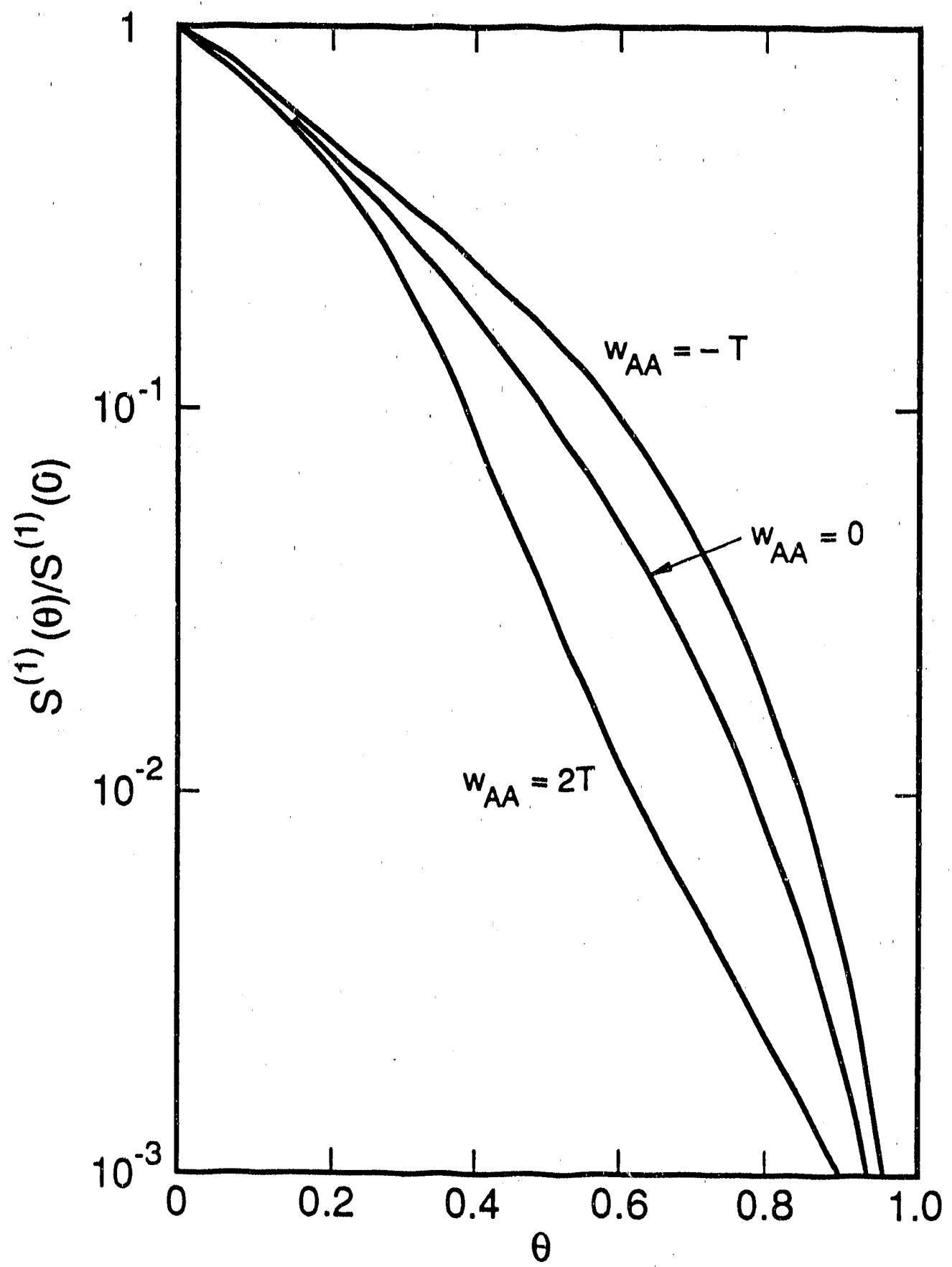

Fig. 3.7 Variation in $S(1)(\theta) / S(1)(0)$ versus $\theta$ when nearestneighbor interactions occur [60]. 

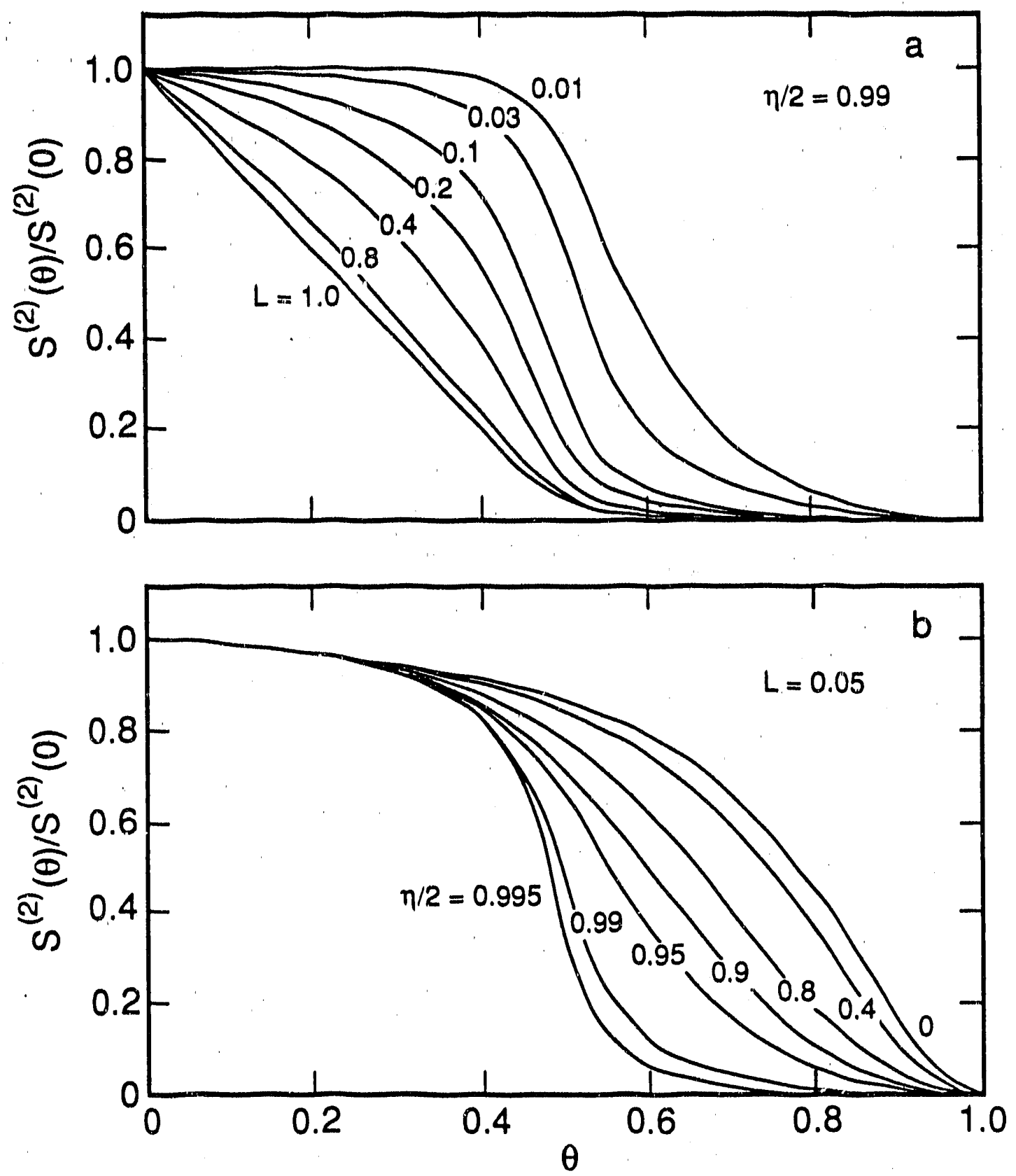

Fig. 3.8 a. Variation in $S(2)(\theta) / S(2)(0)$ versus $\theta$ when precursor states exist for a fixed value of $\eta$. b. Variation in $S(2)(\theta) / S(2)(0)$ versus $\theta$ when precursor states exist for a fixed value of $L[59]$. 
64

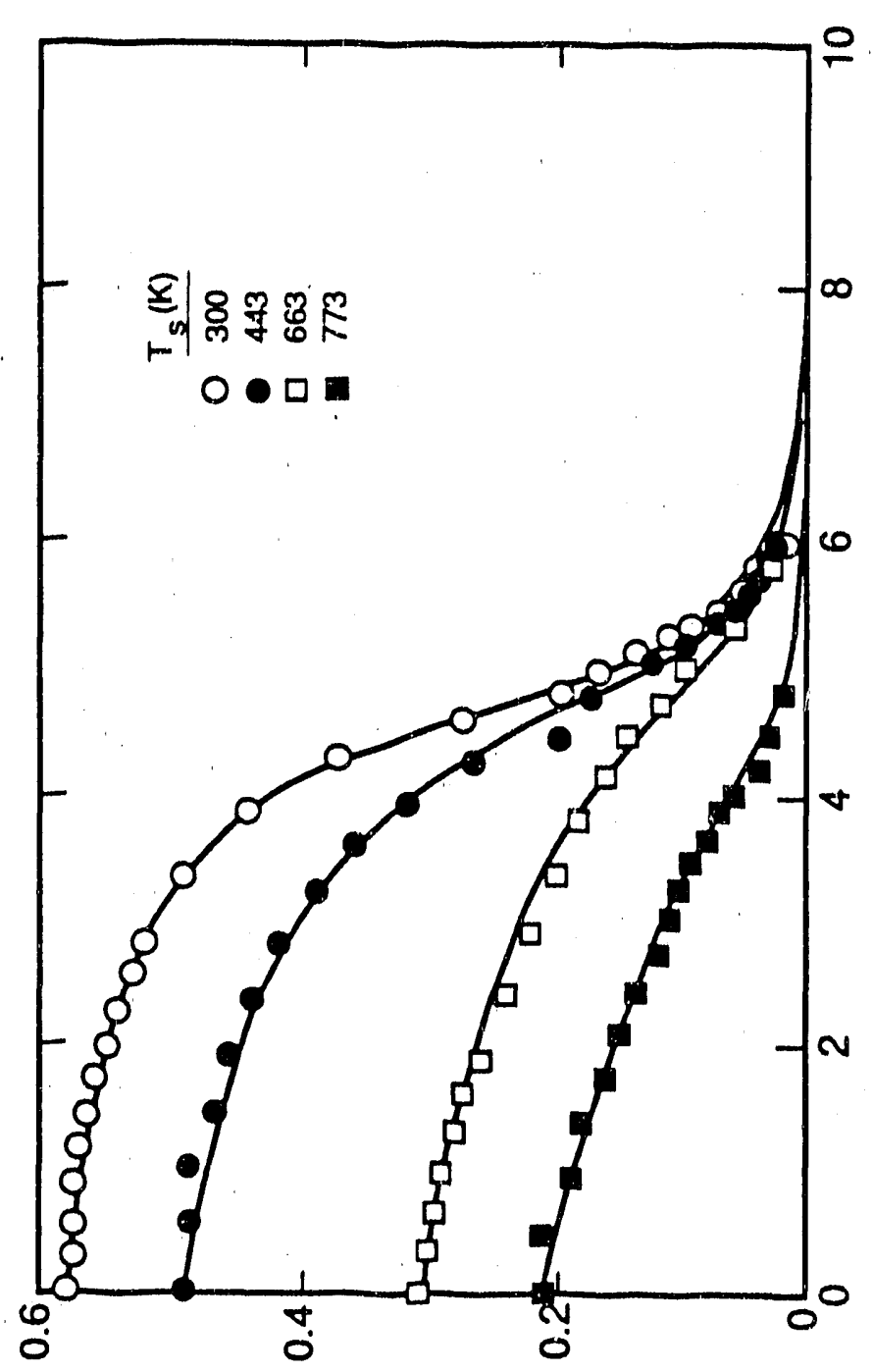

(e) (2) $\mathrm{S}$

ำ

焉

등 응

뜬

$\stackrel{9}{\circ}$

운 

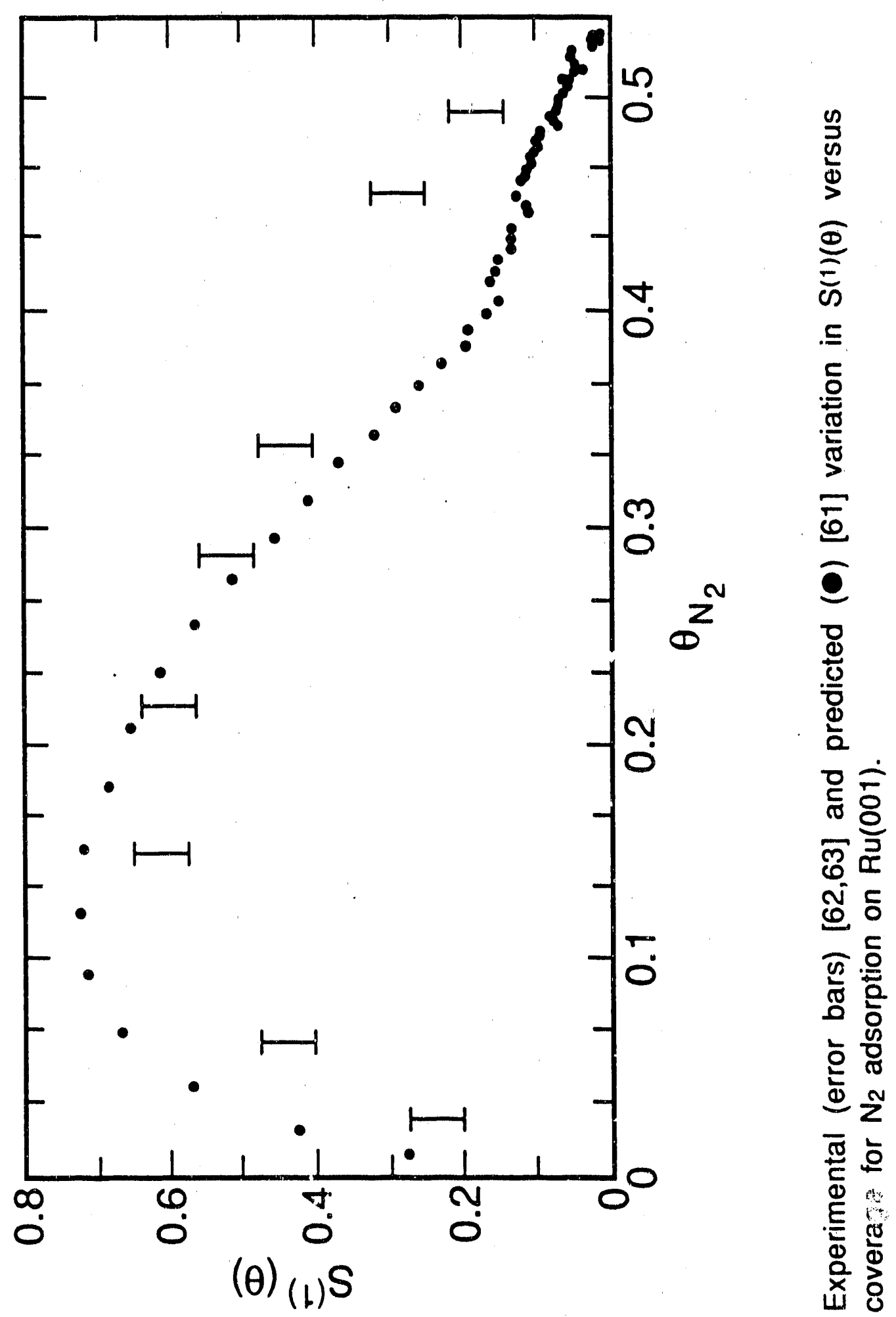

우
i் 


\subsection{DIFFUSION}

\subsection{Molecular Dynamics}

McDowell and Doll [64-67] have used MD simulations to determine the self-diffusivity of $W$ and $R h$ atoms on well-defined surfaces of these metals. The interactions of the adsorbate and surface atoms in all the simu!ations were described by LennardJones potentials, and motion of the top 1-2 layers of the surface was included in the calculations. Table 4.1 lists the predicted and experimental values of $E_{\text {diff }}$ and $D_{0}$. In general, the predicted values of Ediff and $D_{0}$ agree well with the experimentally determined quantities. It was also noted that increasing the number of moving layers from 1 to 2 lead to only a small increase in the calculated diffusivities.

The diffusivities of $C$ and $O$ on $P t(111)$ have been calculated by Doll and Freeman [74] from MD simulations in which the adsorbatemetal interactions were described by Lennard-Jones potentials. The preexponential factor $D_{0}$ and the activation energy Ediff for carbon were determined to be $3.4 \times 10.3 \mathrm{~cm} 2 / \mathrm{s}$ and $26.1 \mathrm{kcal} / \mathrm{mol}$ whereas for oxygen these quant ies were $1.5 \times 10.3 \mathrm{~cm} 2 / \mathrm{s}$ and $18.1 \mathrm{kcal} / \mathrm{mol}$. The predicted values of Ediff are in fair agreement with the experimentally determined values of $31 \pm 4 \mathrm{kcal} / \mathrm{mol}$ for carbon [75] and $16 \mathrm{kcal} / \mathrm{mol}$ for oxygen [76].

Levine and Garofalini [77] have also examined the diffusivity 0 on $\mathrm{Pt}(111)$ witt, an MD approach. The predicted values of $E_{\text {diff }}$ and $D_{0}$ from these simulations agree reasonably with the values determined 
by Doll and Freeman [74] quoted above.

The self-diffusion of Si on Si(100) has been modelled by NoorBatcha et al. [48]. The adsorbate-metal potential energy surface was described by Morse potentials and the interactions between $\mathrm{Si}$ atoms in the lattice were represented by a Keating potential. The values of $D_{0}$ and Ediff for $S i$ diffusion were determined to be $6.35 \pm$ $1.44 \times 10.4 \mathrm{~cm} 2 / \mathrm{s}$ and $3.63 \pm 0.47 \mathrm{kcal} / \mathrm{mol}$. The predicted activation energy is comparable to that determined experimentally, $4.6 \mathrm{kcal} / \mathrm{mol}$ for $\mathrm{Si}$ on $\mathrm{Si}(111)$ [78].

\subsection{Transition-State Theory}

Voter and Doll [79] have used dynamic TST to determine the self-diffusivity of $\mathrm{Rh}$ on $\mathrm{Rh}(100)$. The adsorbate-surface interactions were described by Lennard-Jones potentials and the diffusivities were calculated using eq. 2.85. As shown in fig. 4.1, the predicted and experimentally determined self-diffusivities are in good agreement. The predicted values of $D_{0}$ and $E_{d i f f}$ for $R h$ atoms are $6.6 \pm 0.06 \times 10.3 \mathrm{~cm} 2 / \mathrm{s}$ and $23.82 \pm 0.05 \mathrm{kcal} / \mathrm{mol}$, whereas the experimentally determined quantities are $10.3 \mathrm{~cm} 2 / \mathrm{s}$ and $20.2 \pm 1.7$ $\mathrm{kcal} / \mathrm{mol}$ [69].

The diffusivity of hydrogen on a rigid $\mathrm{Cu}(100)$ surface has been investigated by Valone et al. [80] and Lauderdale and Truhlar [81]. Although the approaches used by these authors were based on classical TST, quantum effects were included in both models. Valone et al. [80] treated all motion classically. Quantum effects were incorporated by use of an effective potential which accounts 
for the effects of zero-point energies and tunneling. Lauderdale and Truhlar [81], on the other hand, treated motion along the reaction coordinate classically, whereas other modes of motion were treated quantum mechanically. The classical motion in the reaction coordinate, however, was multiplied by a semi-classical transmission coefficient to account for zero-point energies and tunneling. In both studies, the inclusion of quantum effects was shown to lead to a non-Arrhenius dependence of the diffusivity at low temperatures. Lauderdale and Truhlar [81] were able to show that the major source of the non-Arrhenius behavior is tunneling. Both Valone [80] and Lauderdale and Truhlar [81] found the ratio of the diffusion coefficient determined from quantum mechanics to that determined from classical mechanics to be of order 103 for $\mathrm{H}$ and 10 for $D$ at $120 \mathrm{~K}$. This level of agreement is remarkable in view of the differences in the formalisms used.

The two models discussed above were extended to examine the influence of the lattice motion on the self-diffusivity of hydrogen. In the work reported by Valone et al. [82], only atoms in the topmost layer were allowed to move, whereas in the work reported by Lauderdale and Truhlar [83] and Truong and Truhlar [84,85], both surface and subsurface atom movement was included. Each of these studies showed that movement of the substrate resulted in larger values of the diffusivity for both $H$ and $D$, the effect being most pronounced at low temperature. At $100 \mathrm{~K}$, the diffusivity for $\mathrm{H}$ on a moving surface was 10 to 103 larger than that for $H$ on a rigid surface.

The diffusion of $\mathrm{H}$ on $\mathrm{Ru}(001)$ in the temperature range 260 - 
$330 \mathrm{~K}$ has been simulated by two groups. In the work of Mak and George [86], the H-surface potential was described by a sum of pairwise-additive Morse potentials whereas in the study by Truong et al. [87], an $a b$ initio potential was used. The values of $D_{0}$ and $E_{\text {diff }}$ determined by Mak and George [86] were $2.2 \times 10.3 \mathrm{~cm}^{2} \mathrm{~s}^{-1}$ and 3.84 $\mathrm{kcal} / \mathrm{mol}$, respectively, whereas the corresponding values calculated by Truong et al. [87] were $10-3 \mathrm{~cm}^{2} \mathrm{~s}^{-1}$ and $4.1 \pm 0.5 \mathrm{kcal} / \mathrm{mol}$. The values of $D_{0}$ predicted in both studies are larger than the experimentally determined value of $D_{0}=6.3 \times 10.4 \mathrm{~cm}^{2} \mathrm{~s}^{-1}[88]$. The value of Ediff determined experimentally is $4.0 \mathrm{kcal} / \mathrm{mol}$ [88] and falls between the two predicted values.

\subsection{Quantum Mechanical Models}

Auerbach et al. [89] have used a quantum mechanical model to treat the diffusion of hydrogen at low coverage on $W(110)$. The model takes into account the effects of phonon-adsorbate interactions on the motion of the adsorbate. At low temperature, the adsorbate motion arises from phonon-assisted tunneling whereas at high temperature, the motion occurs by an activated hopping process. Figure 4.2 shows the predicted and experimentally determined $[90,91]$ diffusivities as a function of temperature. It can be seen that the model successfully represents the experimentally observed trends. The model developed by Auerbach et al. [89] also predicts that in the activated diffusion regime, the preexponential factor for diffusion increases exponentially with increasing mass of the adsorbate, a trend which, although opposite to the classical 
isotope dependence, is in accord with the experimentally observed mass dependence of the preexponential factor for hydrogen on $W(110)$ [91].

The coverage duperidence of the diffusivity of hydrogen on $W(110)$ has been deterriviried by Whaley et a!. [92] using a model which accounts for the band structure of hydrogen atoms in a periodic surface potential. The model differentiates between lowand high-coverage diffusion. At low coverage, the hydrogen motion is described by a random collision process in a uniform potential, whereas at high coveage, the hydrogen motion is affected by the perturbations of nearest-neighbor $\mathrm{H}-\mathrm{H}$ interactions on the surface potential. The predicted diffusivities for the isotopes of hydrogen are shown in fig. 4.3a and display a complex coverage dependence. For comparison, the experimentally observed diffusivity profiles with coverage are shown in fig. 4.3b [91]. With the exception of the deuteron profile at high coverage, the model predictions agree qualitatively with the experimental results.

\subsection{Effects of Lateral Interactions}

The occurrence of lateral interactions between species can influence the magnitude of the activation energy barrier for diffusion as well as the distribution of adsorbates on the surface. Lateral interactions between adsorbates are also responsible for ordered adsorbate overlayers, island formation, and order-disorder phase transitions. Both continuum, lattice-gas models and Monte Carlo models have been developed to determine the effects of lateral 
interactions on the diffusion of adsorbates on surfaces.

Continuum Models

Reed and Ehrlich [93] have investigated the influence of lateral interactions on the coverage dependence of the diffusivity. In this work, the flux was expressed in terms of the chemical potential gradient:

$J=-\Gamma(\theta) \lambda^{2}\left[\frac{1}{1-\theta}+\left(\frac{\partial \mu / k_{b} T}{\partial \ln \theta}\right)_{T}\right] \frac{\partial C}{\partial x}$

where $\mu$ is the chemical potential. The coverage-dependent diffusivity was then given by $D(\theta)=\Gamma(\theta) \lambda^{2}\left[\frac{1}{1-\theta}+\left(\frac{\partial \mu / k_{b} T}{\partial \ln \theta}\right)_{T}\right]$

Both $\Gamma(\theta)$ and $\mu$ were determined using a lattice-gas model with the quasi-chemical approximation. Calculations of $D(\theta) / D(0)$ were made for selected values of the lateral interaction parameter $W A A=k T^{*}$, where $T^{*}$ is the reference temperature. As seen in fig. 4.4 for repulsive interactions, $D(\theta) / D(0)$ increases with increasing $\theta$ up to $\theta$ $=0.8$ and then decreases weakly. Although not shown here, for attractive lateral interactions, $D(\theta) / D(0)$ decreases rapidly from a value 1.0 at $\theta=0$ to a value of 0.05 at $\theta=0.5$ and then remains relatively constant at this value to $\theta=1.0$.

Using an approach similar to that described above, Zhdanov [94] has examined how both nearest- $\left(w_{1}\right)$ and next-nearest-neighbor interactions ( $\left.w_{2}\right)$ influence the diffusivity. As seen in fig. 4.5, $D(\theta) / D(0)$ goes through a maximum with increasing $\theta$. The location of the maximum is sensitive to the magnitude of the $w_{2}$. In a related 
study, Zhdanov [95] has treated the coverage dependence of the diffusion coefficient when two different species, $A$ and $B$, are coadsorbed. This study demonstrated that $D(\theta) / D(0)$ is sensitive to both the magnitude and sign of the interactions between $A$ and $B$. Monte Carlo Models

The diffusive motion of an ensemble of adsorbates can be described using Monte Carlo techniques. The adsorbate atoms or molecules are assumed to be located at fixed positions on a lattice of surface sites. The hopping of adsorbates from site $i$ to site $j$ can be described by $\mathrm{P}_{\mathrm{ij}}$, a transition probability which depends on the configuration of nearest-neighbor sites surrounding the initial and final sites. Simulations begin by first choosing an adsorbate at random and then selecting one of the $z$ nearest-neighbor sites. If the neighboring site is occupied, the procedure is begun again. If the neighboring site is unoccupied, the hopping probability $P_{i j}$ is computed and compared to a random number $R(0 \leq R \leq 1)$. The adsorbate is moved from site $i$ to site $j$ if $R \leq P_{i j}$, otherwise it remains in its original position. Each time an adsorbate is selected, the time is increased by an amount $\Delta t=\tau / N$, where $1 / \tau$ is the attempt frequency of hops and $\mathrm{N}$ is the number of adsorbates in the ensemble. The surface diffusion coefficient, $D$, is calculated from the ensemble average of the mean-square displacement of adsorbates using eq. 2.72 .

The transition probability $P_{i j}$ disclissed above can be described in terms of an activation energy for diffusion, Ediff, or an energy difference between sites $i$ and $j, \Delta E=\left(Q_{j}-Q_{i}\right)$. In the presence of lateral interactions, both $E_{d i f f}$ and $\Delta E$ depent on the configuration of 
nearest-neighbor sites surrounding the initial and final sites. If adsorbate diffusion is characterized by $E_{\text {diff, }} P_{i j}$ is given by

$P_{i j}=\exp \left[-E_{d i f f} / k_{b} T\right]$

If the representation of adsorbate diffusion is in terms of the energy difference $\Delta E, P_{i j}$ is described by either Metropolis [96] or Kawasaki [97] cynamics. For Metropolis dynamics,

$$
\begin{aligned}
P_{i j} & =\exp \left[\frac{\Delta E}{k_{b} T}\right] & & \text { for } \Delta E<0 \\
& =1 & & \text { for } \Delta E \geq 0
\end{aligned}
$$

For Kawasaki dynamics,

$$
P_{i j}=\frac{\exp \left[\Delta E / k_{b} T\right]}{1+\exp \left[\Delta E / k_{b} T\right]}
$$

Kang and Weinberg [98] have recently discussed the proper specification of $P_{i j}$ and $\tau$ required to correctly represent the dynamics of diffusion. Their analysis shows that $P_{i j}$ must be written as given in eq. 4.3 and $\tau=1 / v_{\text {diff, }}$ where $v$ diff is the frequency of frustrated translation motion parallel to the surface. It was also shown that specification of $\mathrm{P}_{\mathrm{ij}}$ using either Kawasaki or Metropolis dynamics does not produce a proper description of the surface dynamics. This is demonstrated in fig. 4.6 which shows plots of $D$ versus $1 / T$ for two representations of $P_{i j}$. In one case, $P_{i j}$ is given by the energy barrier mudel (eq. 4.3) whereas in the other, $P_{i j}$ is given by Kawasaki dynamics (eq. 4.5). As seen in fig. 4.6, the two models lead to different dependences of the diffusivity with temperature. Kang and Weinberg [98] indicate, however, that the diffusivities determined from Kawasaki dynamics can be brought into agreement with those determined from the energy barrier model by rescaling $\tau$. 
The diffusivities determined from the rescaled Kawasaki model are also shown in fig. 4.6 and are seen to be in good agreement with the diffusivities predicted from the energy barrier model. In general, though, the rescaling factor is not known and thus accurate diffusivities can only be obtained from the energy barrier model.

Models in which eqs. 4.3-4.5 are used to determine the coverage dependence of the diffusivity are discussed next. Bowker and King [99] have used a Monte Carlo model to investigate the influence of lateral interactions on the diffusivity. Lateral interactions were described in a pairwise additive manner. Concentration gradients were established on a two-dimensional array and jumps of adsorbates between sites were monitored as a function of coverage and time. Coverage-dependent diffusivities were then obtained for the three cases of repulsive, attractive, and no interactions between adsorbates. As seen in fig. 4.7, D increases with increasing $\theta$ for repulsive interactions, whereas it decreases with increasing $\theta$ for attractive interactions. In the absence of lateral interactions, $D$ is coverage independent. Bowker and King [100] have also examined the influence of repulsive nearest-neighbor and attractive next-nf rest neighbor interactions on $D$. Figure $4.8 \mathrm{a}$ shows that the predicted profile of $D$ goes through a maximum near $\theta$ $=0.45$. This type of coverage dependence is qualitatively similar to that observed experimentally for oxygen diffusion on $W(110)$, which is shown in fig. $4.8 \mathrm{~b}$ [101].

Tringides and Gomer [102] have also simulated the diffusion of oxygen on $W(110)$ using a Monte Carlo model. Both pairwise and triplet interactions between adsorbates were considered. The 
experimentally observed [103] increase in the activation energy and preexponential factor with increasing coverage were accounted for with nearest-neighbor interactions of $2.1 \mathrm{kcal} / \mathrm{mol}$, next-nearestneighbor interactions of $-0.7 \mathrm{kcal} / \mathrm{mol}$, and triplet interactions of 1.2 or $0 \mathrm{kcal} / \mathrm{mol}$.

The effects of adsorbate coverage on the diffusion coefficient for ordered or disordered adsorbates have been modelled by Sadiq and Binder [104]. Figure 4.9a shows that for disordered adsorbates, the diffusivity goes through a maximum as a function of coverage. Simulations were also conducted for a case in which the adsorbates can form an ordered overlayer at $\theta=0.5$. As seen in fig. $4.9 b$, the diffusion coefficient exhibits maxima at $\theta=0.33$ and $\theta=0.67$. The minimum at $\theta=0.5$ is attributed to the formation of an ordered surface overlayer which causes a reduction in the diffusivity. Mak et al. [105] have examined the effect of an immobile species $B$ on the diffusivity of a mobile species $A$ assuming no lateral interactions. Figure 4.10a displays the dependence of $D\left(\theta_{A}\right) / D(0)$ on $\theta_{B} . D\left(\theta_{A}\right) / D(0)$ is seen to decrease with increasing coverage of $B$. On the other hand, $D\left(\theta_{A}\right) / D(0)$ decreases linearly with $\theta_{B}$, as shown in fig. $4.1 \mathrm{Cb}$. The intercept with the abscissa at $\theta_{B}=$ 0.408 is in agreement with percolation estimates for the coverage of $B$ above which no further diffusion of $A$ can occur. 
Table 4.1

Arrhenius parameters for atomic self-diffusion [64]

\begin{tabular}{|c|c|c|c|c|}
\hline \multirow[t]{2}{*}{ Atom/Surface } & \multicolumn{2}{|c|}{$\mathrm{Do}_{0}\left(\mathrm{~cm}^{2} / \mathrm{s}\right)$} & \multicolumn{2}{|c|}{$E_{\operatorname{difl}}(\mathrm{kcal} / \mathrm{mol})$} \\
\hline & Exp. & Calc. & Exp. & Calc. \\
\hline \multirow[t]{3}{*}{$W / W(110)$} & $22.1 \times 10.3$ & & a) 9.8 & \\
\hline & $62.6 \times 10.3$ & & b21.2 & \\
\hline & $6.2 \times 10^{-3}$ & & 120.8 & \\
\hline Average & $3.6 \times 10.3$ & $3.59 \pm 1.77 \times 10.3$ & 20.6 & $22.7 \pm 3.0$ \\
\hline \multirow[t]{4}{*}{$W / W(211)$} & $\mathrm{d} 3.0 \times 10.4$ & & $d 17.5$ & \\
\hline & $01.2 \times 10.4$ & & 016.8 & \\
\hline & $12.0 \times 10.2$ & & 119.7 & \\
\hline & $12.0 \times 10.3$ & & 117.8 & \\
\hline Average & $5.6 \times 10.3$ & $2.18 \pm 2.72 \times 10.3$ & 18.0 & $20.5 \pm 5.2$ \\
\hline $\operatorname{Rh} / \operatorname{Rh}(111)$ & $b 2 \times 10.4$ & $7.10 \pm 1.28 \times 10.4$ & b3. 6 & $6.2 \pm 0.6$ \\
\hline$R h / R h(100)$ & $01 \times 10.3$ & $4.06 \pm 2.34 \times 10.3$ & $b_{2} 0.2$ & $20.8 \pm 3.7$ \\
\hline
\end{tabular}

aFrom Ref. [68]. bFrom Ref. [69]. cFrom Ref. [70]. dFrom Ref. [71]. eFrom Ref. [72]. IFrom Ref. [73]. 


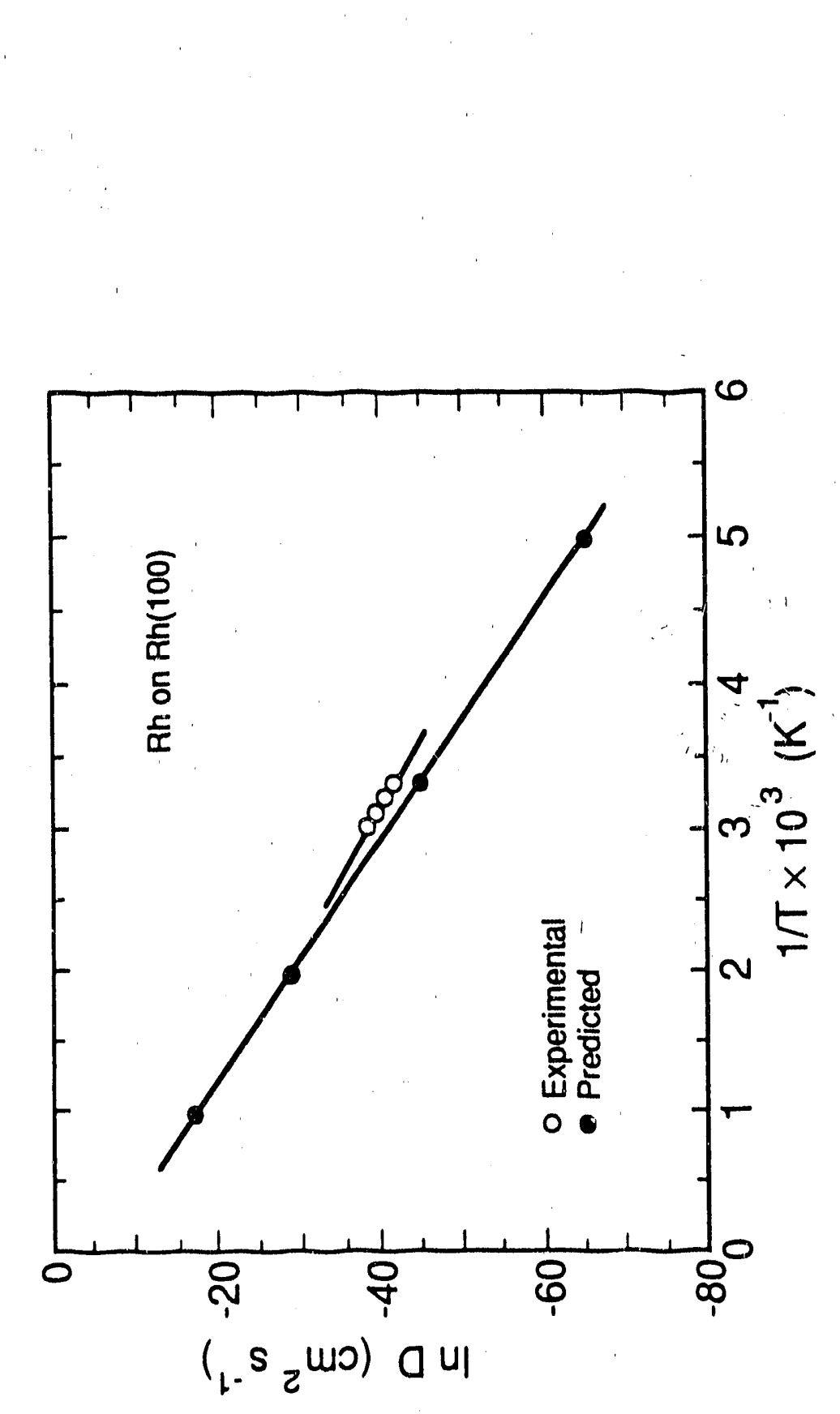

등

돔

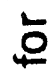

○

$E$

क

ఏ

D

. 들

高

D

든

ఠ్

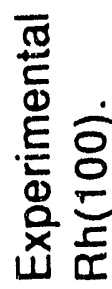

$\dot{j}$
$\dot{0}$ 


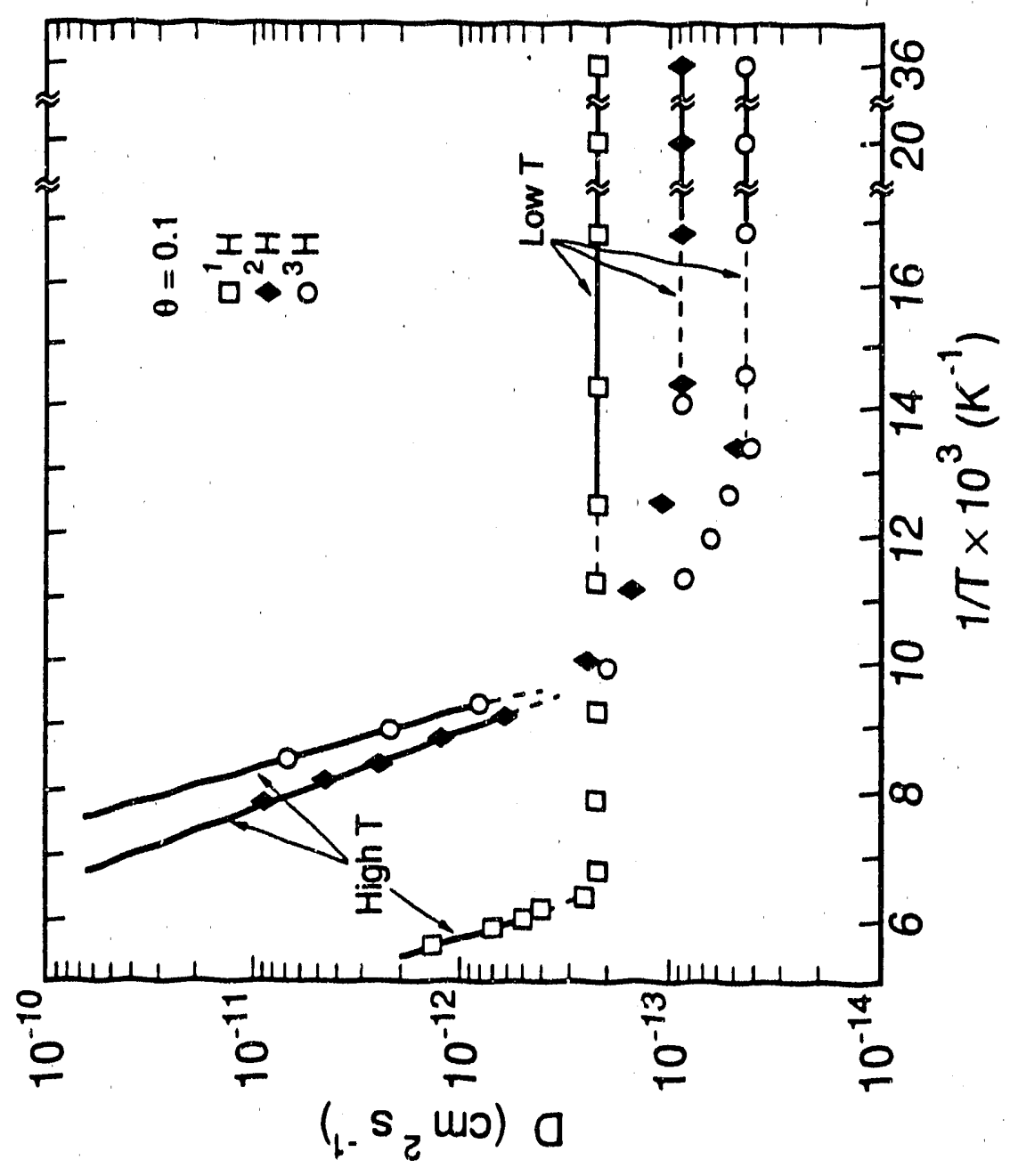

$\stackrel{Ð}{=}$

ஸั

(s)

응

के

.

음

$\bar{\Phi}$

ङ

ᄃㅇํ

은

क्ष

등

ठั่

늠

초음

흠

5 号

索

드

它

$\stackrel{y}{*}$

i⿱宀 


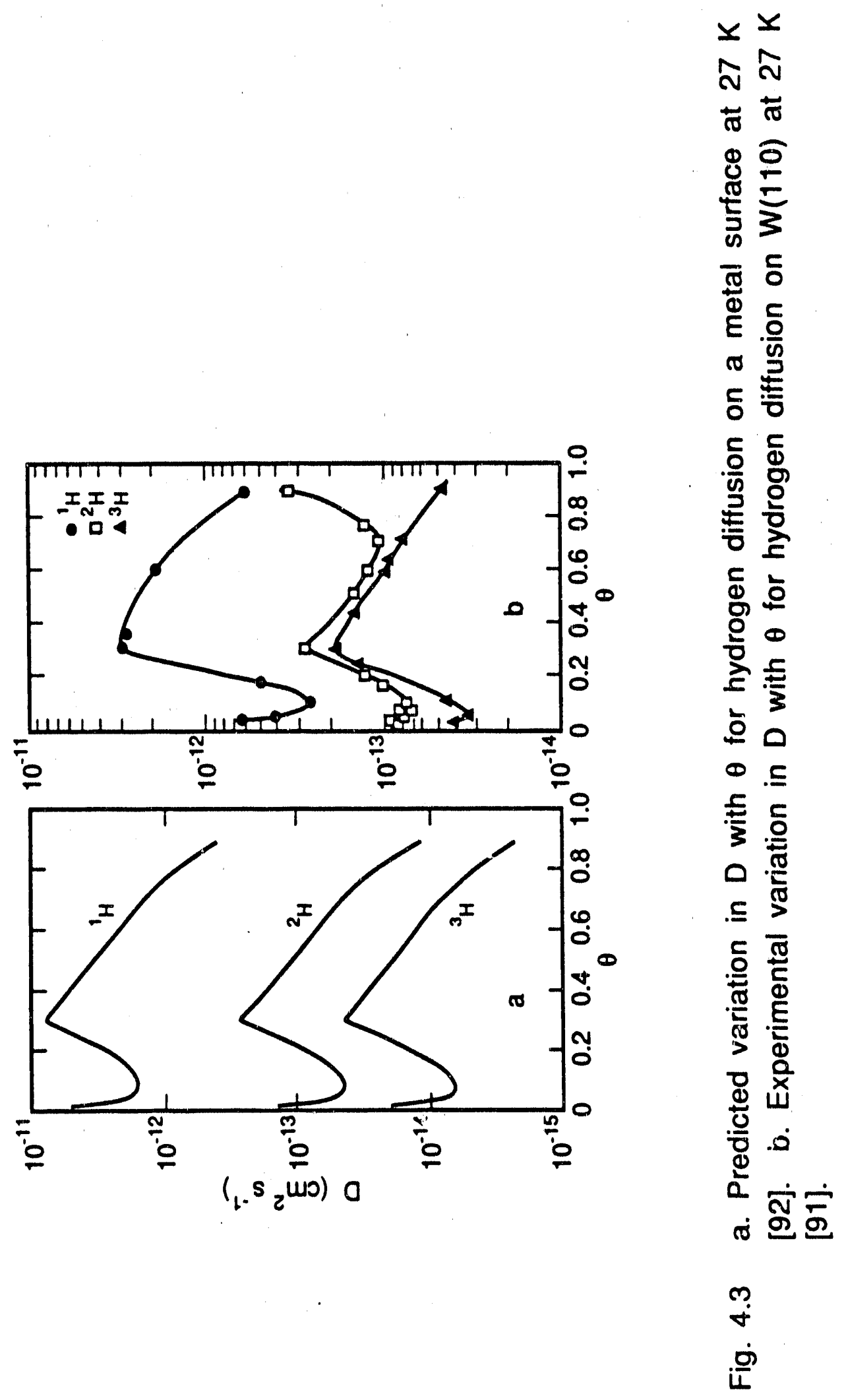




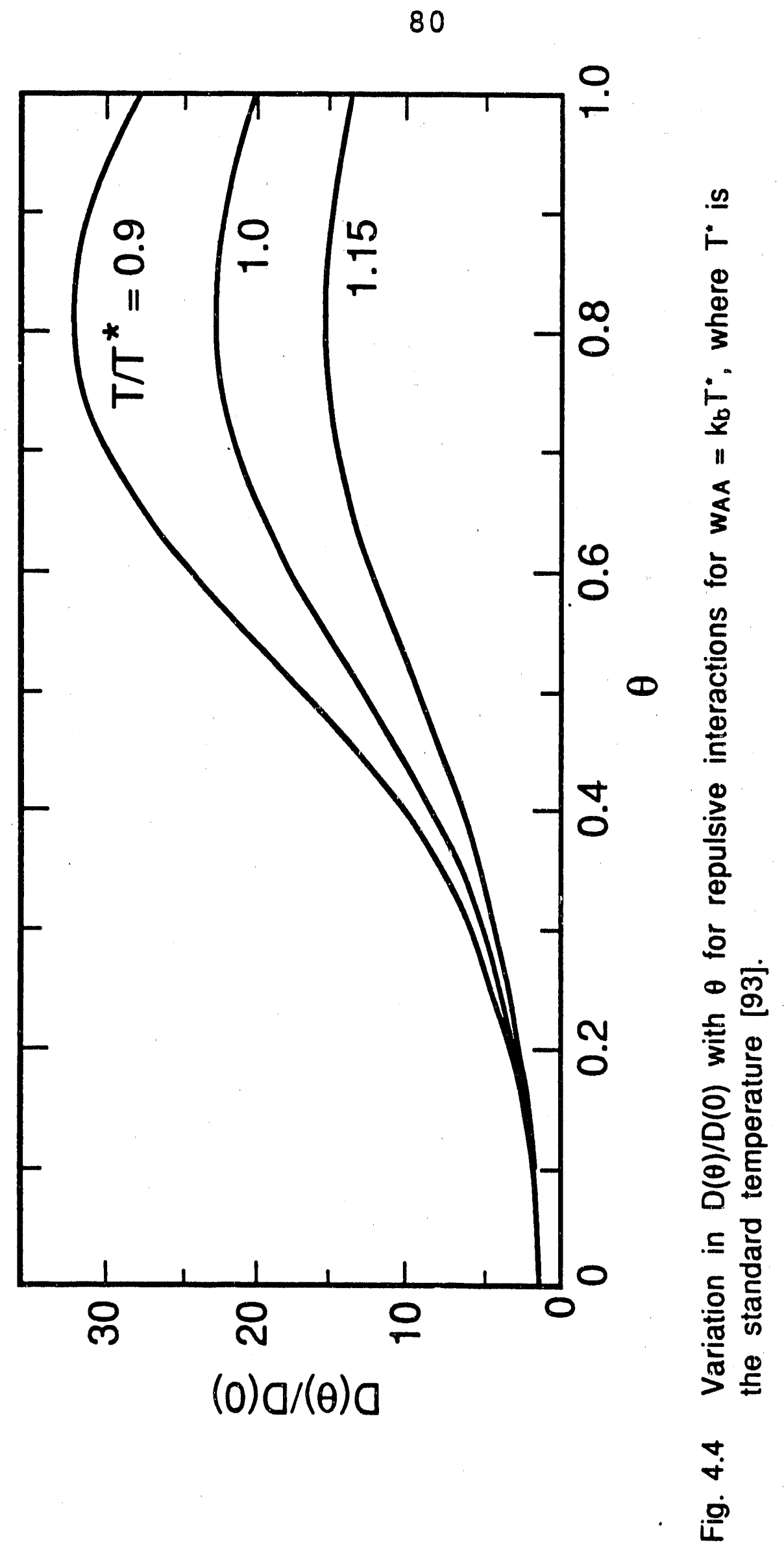




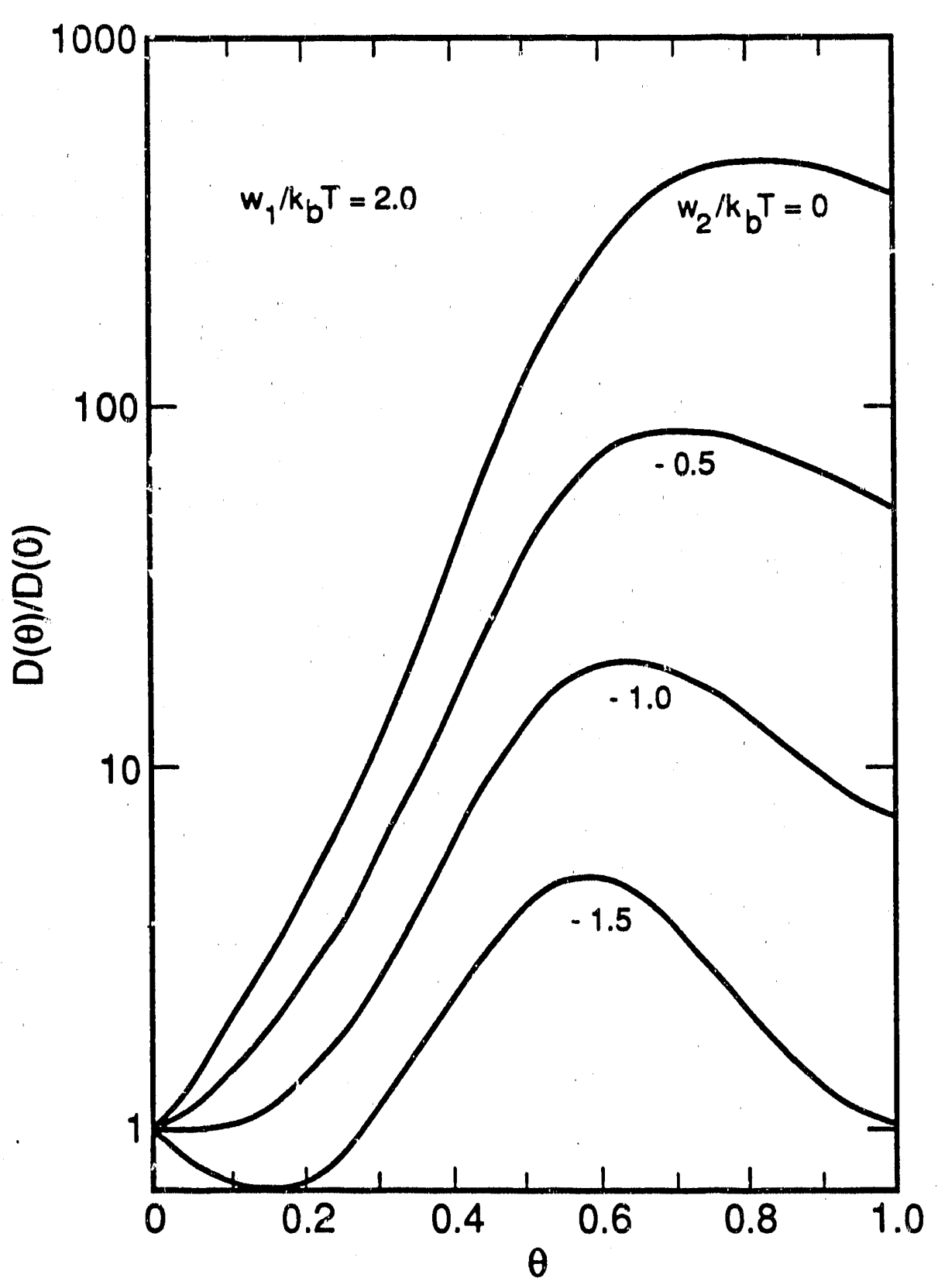

Fig. 4.5 Variation in $D(\theta) / D(0)$ with $\theta$ for nearest-( $\left.w_{1}\right)$ and nextnearest-(w2) neighbor interactions [94]. 

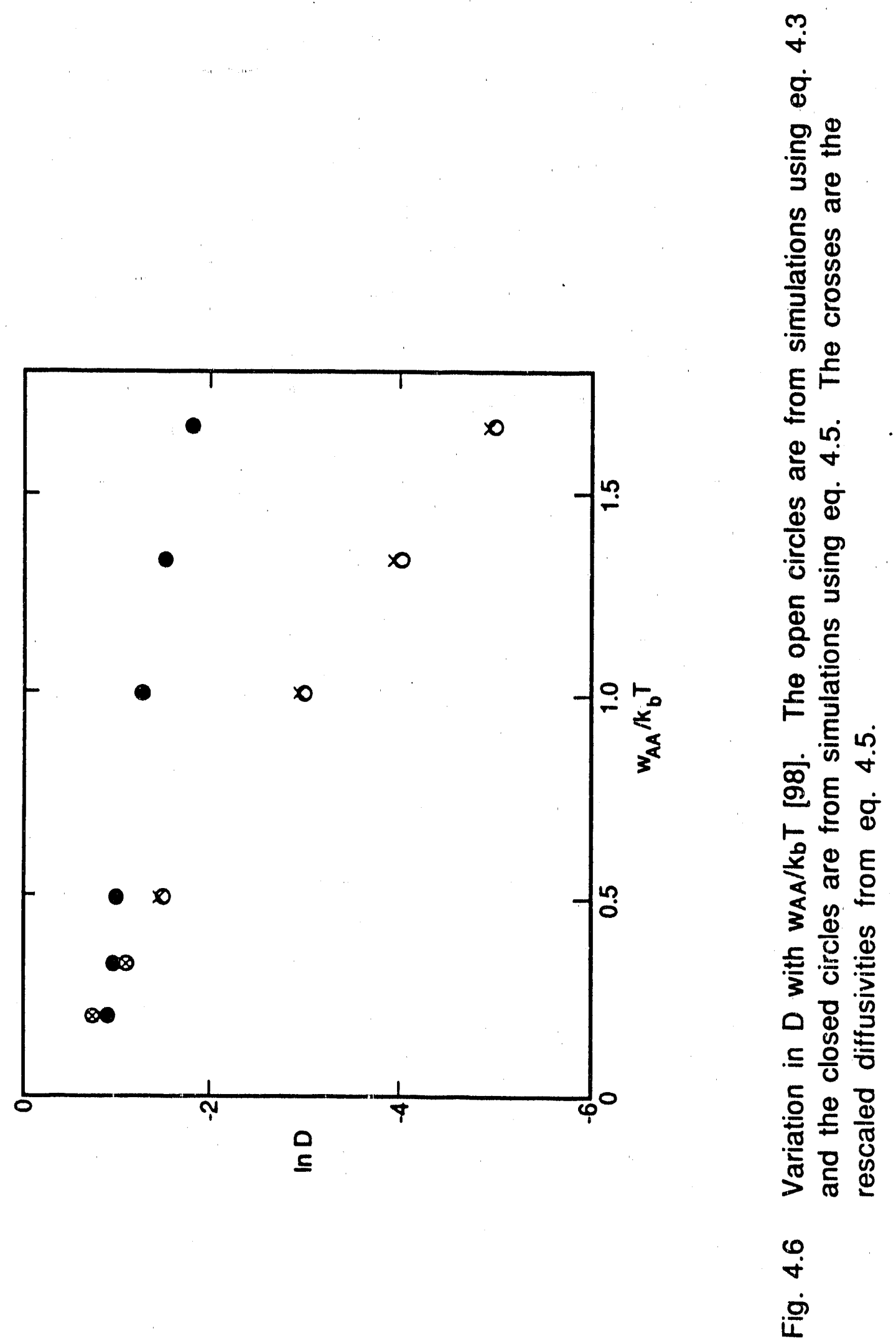

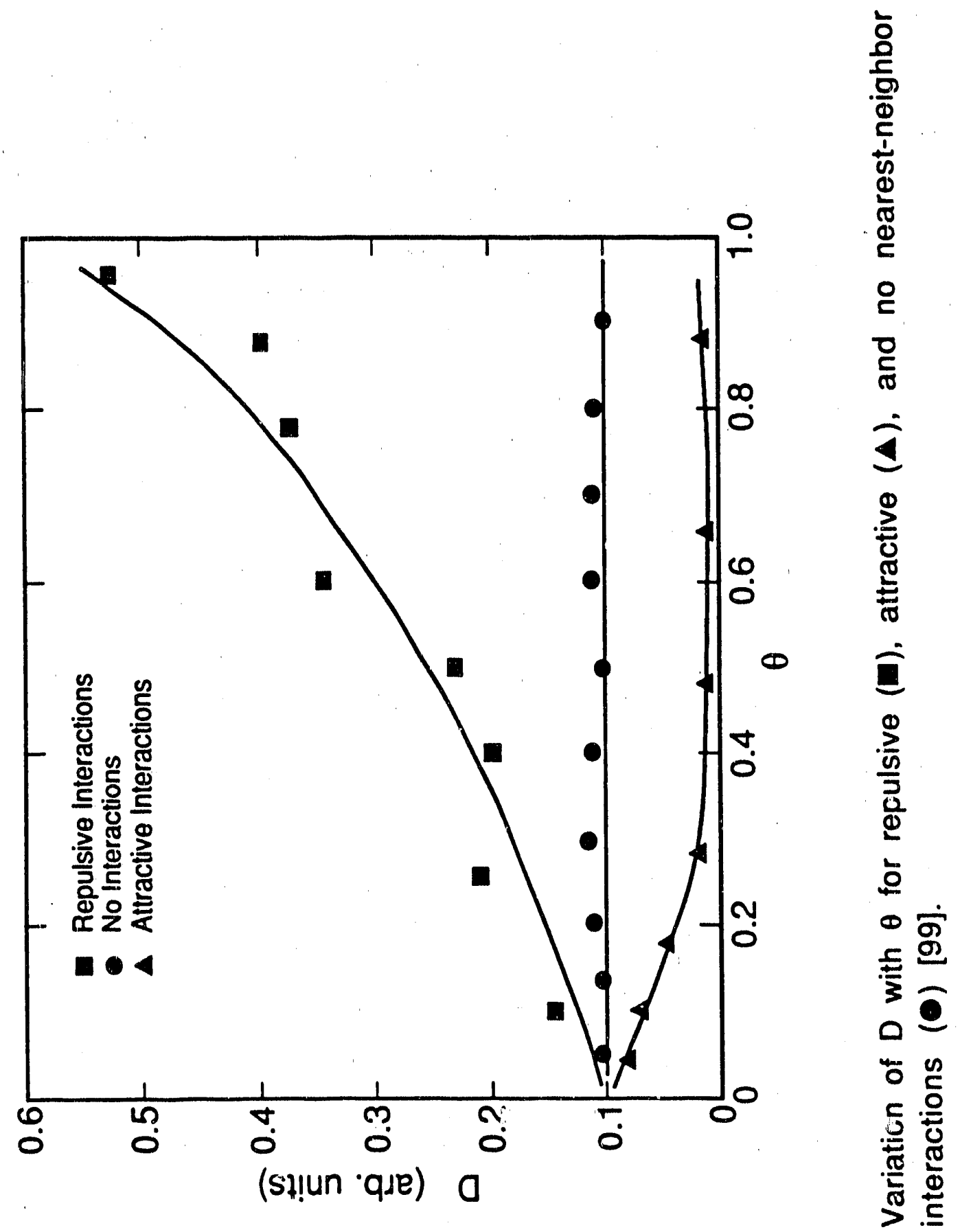

字 

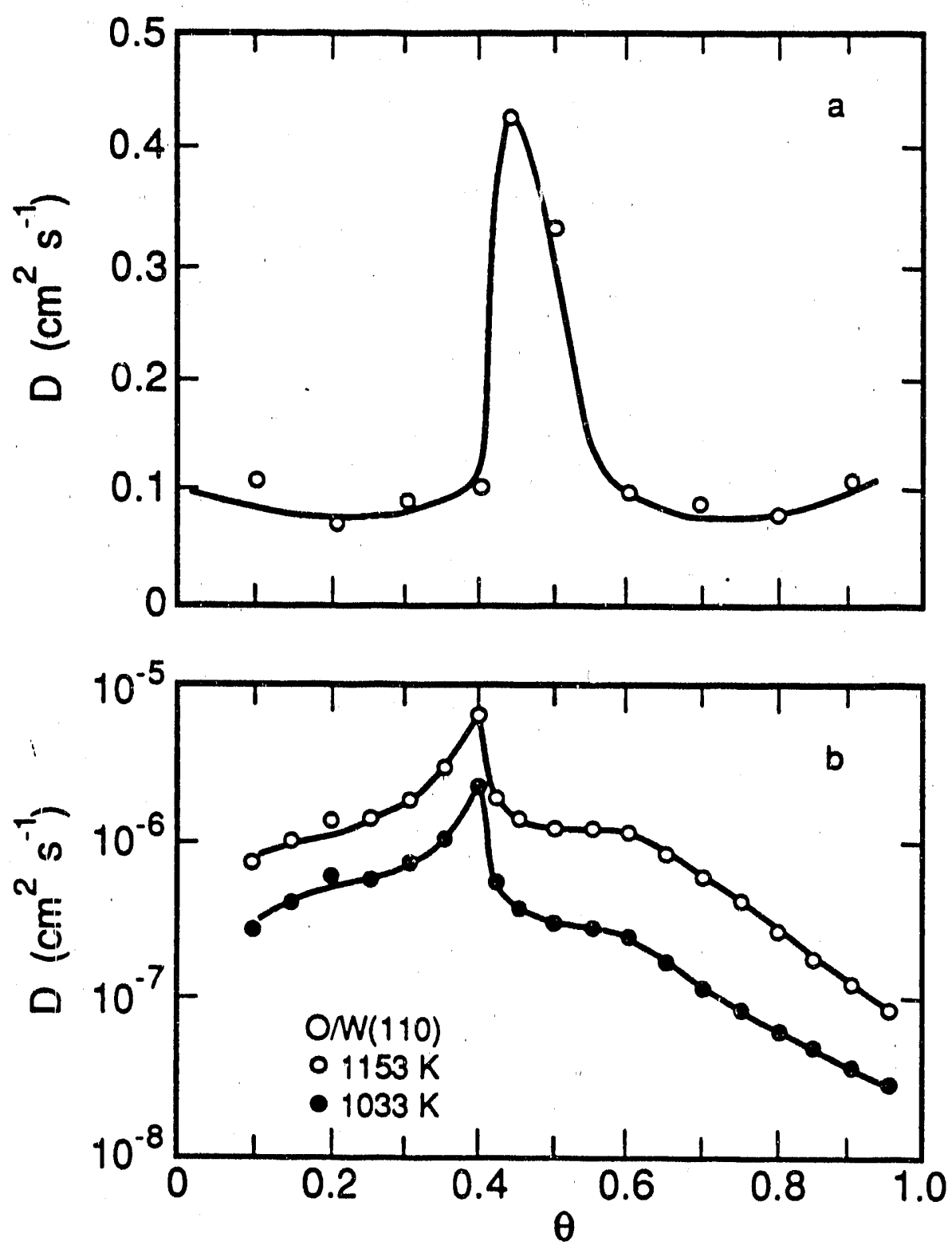

Fig. 4.8 a. Simulation of the $\theta$ dependence of $D$ for repulsive nearest-neighbor and attractive next-nearest neighbor interactions [100]. b. Experimental variation in $D$ with $\theta$ for oxygen on $W(110)$ [101]. 


\section{5}
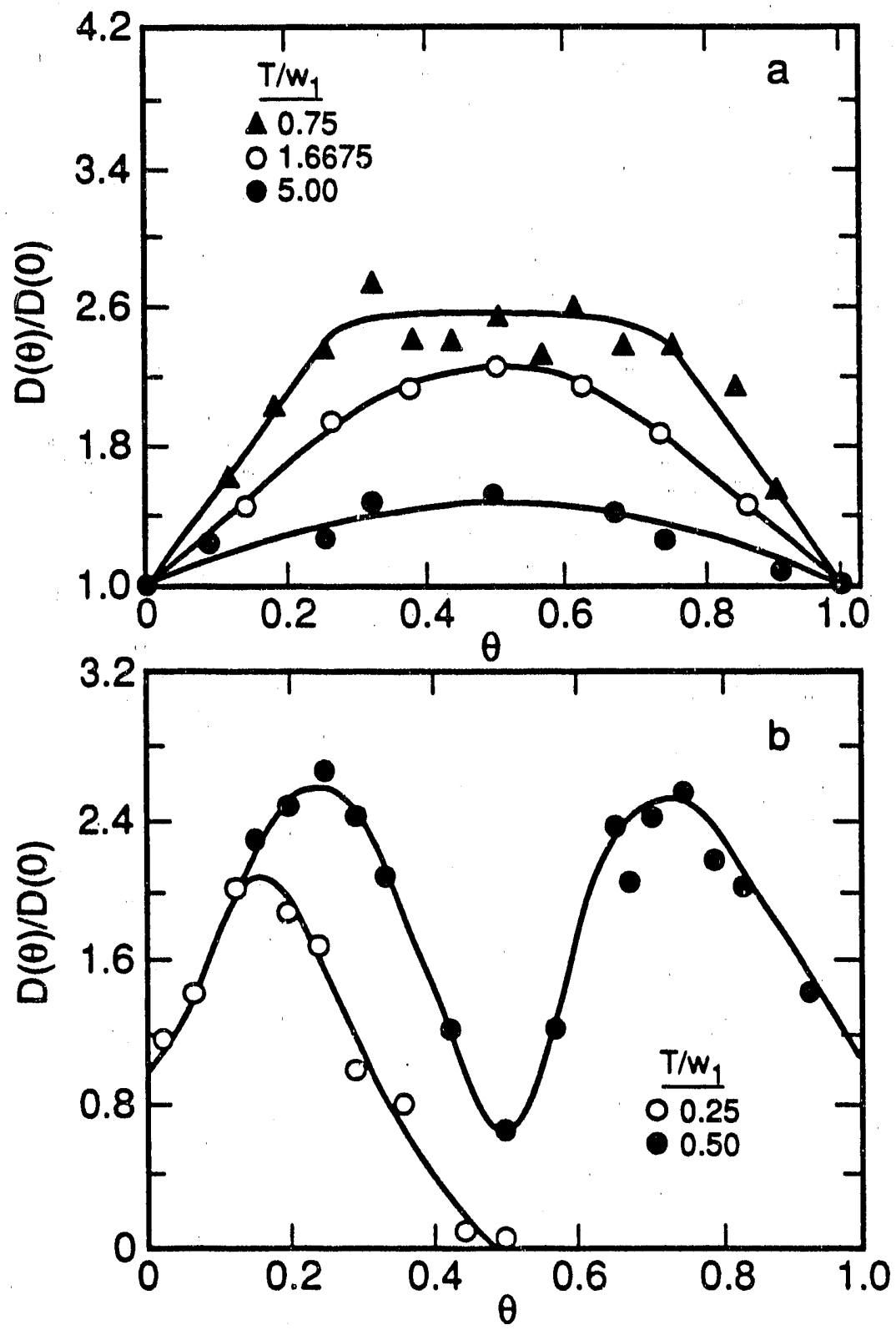

Fig. 4.9 Variation in $D(\theta) / D(0)$ with $\theta$ for equivalent nearest- $\left(w_{1}\right)$ and next-nearest-( $\left.w_{2}\right)$ neighbor repulsive interactions [104]. a. Disordered region. b. Ordered region. 
86
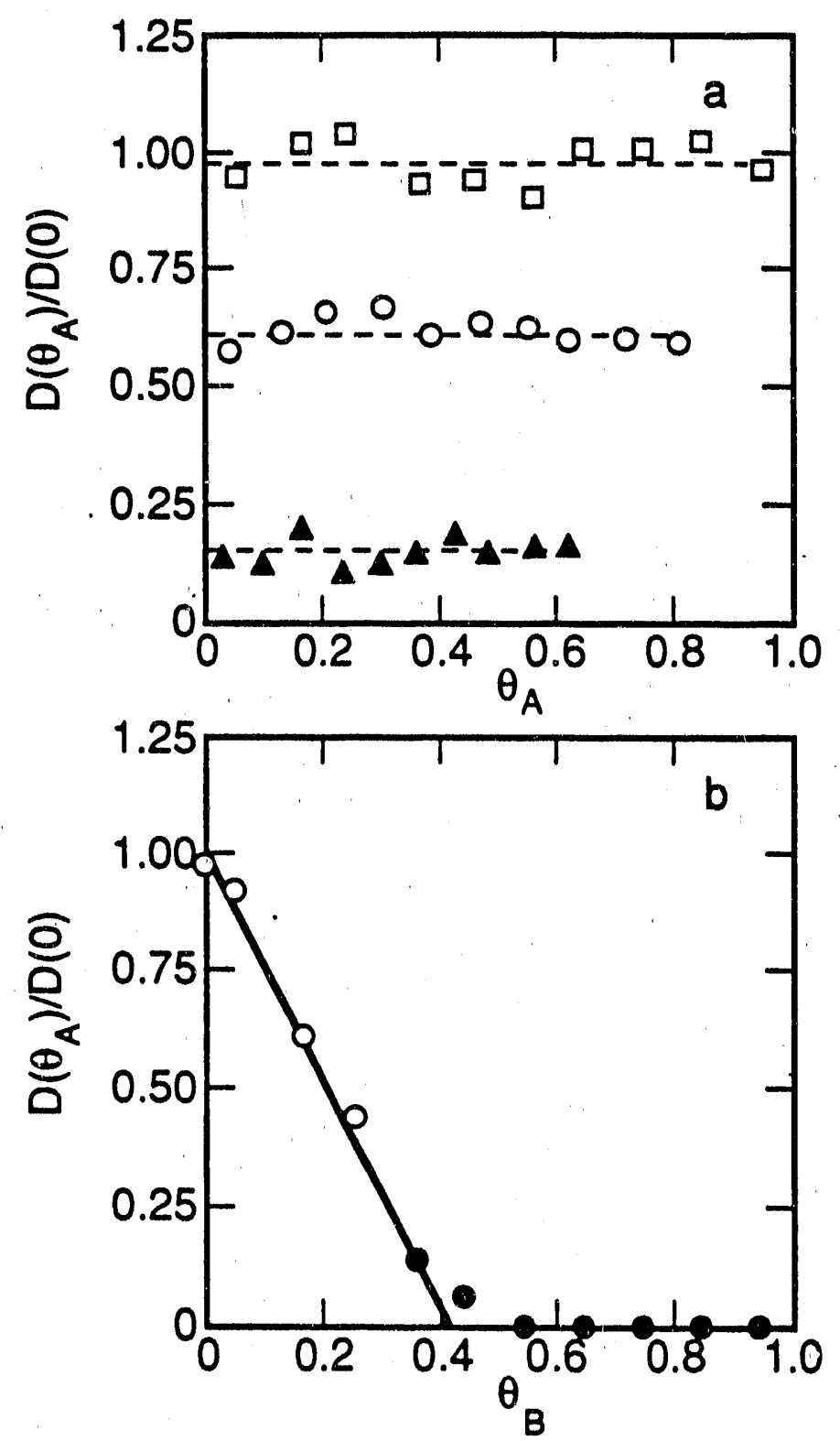

Fig. 4.10 a. Variation in $D\left(\theta_{A}\right) / D(0)$ with $\theta_{A}$ in the presence of an immobile adsorbate $B$ for $\theta_{B}=0(\square), \theta_{B}=0.15(0)$, and $\theta_{B}=0.36(\triangle)$. b. Variation in $D\left(\theta_{A}\right) / D(0)$ with $\theta_{B}$. The filled symbols represent upper limits [105]. 


\subsection{DESORPTION}

\subsection{Transition-State and Stochastic Diffusion Theory}

Tully and coworkers $[28,29]$ have calculated the rate coefficients for $\mathrm{Xe}$ and $\mathrm{Ar}$ desorption from $\mathrm{Pt}(111)$ using both dynamically corrected (eq. 2.75) and classical (eq. 2.76) TST. The results of these salculations are illustrated in fig. 5.1. At low temperatures, both approaches give identical results, indicating that is goes to 1.0 as the temperature decreases. It is also observed that at low temperatures, $k_{d}(1)$ exhibits Arrhenius behavior. The apparent activation energies for $\mathrm{Ar}$ and $\mathrm{Xe}$ desorption are 8.8 and $28.8 \mathrm{~kJ} / \mathrm{mol}$, respectively, in good agreement with the experimental values of 9.2 and $29.3 \mathrm{~kJ} / \mathrm{mol}[106,107]$. In the limit of low temperatures, the apparent preexponential factors for $\mathrm{Ar}$ and $\mathrm{Xe}$ are $5.2 \times 1011$ and 6.8 $\times 1011 s^{-1}$, respectively, which agree approximately with the vibrational frequencies of the rare-gas atoms on the surface: $9.8 \times$ $1011 \mathrm{~s}^{-1}$ for $\mathrm{Ar}$ and $9.2 \times 1011 \mathrm{~s}^{-1}$ for $\mathrm{Xe}$. Figure 5.1 shows that with increasing temperature above $200 \mathrm{~K}$, the value of $\mathrm{kd}_{d}(1)$ predicted by dynamically corrected TST becomes progressively smaller than that calculated by classical TST. This deviation is a reflection of the decreasing value of $f_{s}(T)$ [i.e., $S(1)(0)$ ] as $T$ increases. The nonArrhenius behavior of $k_{d}(1)$ is explained in the following way. At high temperatures, the mean energy transferred upon impact of the rare gas atom with the surface is relatively small and so consequently equilibrium can no longer be maintained among adsorbates located near the top of the potential well. This causes a 
depletion in the population of the precursor levels due to desorption into the gas phase and hence, to a reduction in the rate of desorption.

Using an approach similar to that described above, Muhlhausen et al. [47] have examined the molecular desorption of NO from $\mathrm{Pt}(111)$. The rate of NO desorption exhibited Arrhenius behavior at low temperature, but at temperatures above $1000 \mathrm{~K}$, curvature was observed. This high temperature behavior was explained in the same manner as that for $X_{e}$ and $A r$ desorption, discussed above. The preexponential factor determined from the low temperature portion of the Arrhenius plot was $1016 \pm 0.4 \mathrm{~s}^{-1}$, in excellent agreement with the experimental value of $1016 \mathrm{~s}^{-1}$ [108]. This value of $v_{d}(1)$, which is larger than the value of $1013 \mathrm{~s}^{-1}$ often assumed, is ascribed by the authors to the reduced entropy of chemisorption associated with the alignment of the molecule perpendicular to the surface.

Doren and Tully [36] have calculated $k_{d}(1)$ for $C O$ desorption from $\mathrm{Ni}(111)$ using dynamic TST. Figure 5.2 illustrates plots of the apparent Arrhenius parameters $E_{d}$ and $v_{d}(1)$ versus temperature. Both the activation energy and the preexponential factor are seen to be temperature dependent and to decrease with increasing temperature. For temperatures between 400 and $600 \mathrm{~K}, \mathrm{vd}(1)$ is in the range of 1014 to $1015 \mathrm{~s}^{-1}$. The explanation for this large value of $\mathrm{vd}^{(1)}$ for $\mathrm{CO}$ is identical to that presented above for NO.

The associative desorption of $\mathrm{H}_{2}$ from $\mathrm{Si}(111)$ has been modelled by Raff et al. [109] with a variational TST approach. The potential energy surface. was defined by the sum of three terms corresponding to the lattice potential, adatom-lattice interactions, and adatom-adatom interactions. The rate coefficient $k_{d}(2)$ exhibited 
Arrhenius behavior over the temperature range investigated. The apparent activation energy and preexponential factor were 55 $\mathrm{kcal} / \mathrm{mol}$ and $0.20 \mathrm{~cm}^{2} \mathrm{~s}^{-1}$, respectively. These values lie within the range of the experimentally observed values of $41-59 \mathrm{kcal} / \mathrm{mol}$ for the activation energy and $0.03-136 \mathrm{~cm}^{2} \mathrm{~s}^{-1}$ for the preexponential factor [110-112].

Stochastic diffusion theory has been used by Zeiri et al. [41] to describe the desorption of $K$ and $X e$ from a $W(111)$ surface arid the molecular desorption of $\mathrm{rO}$ from a Ni(110) surface. The values of the parameters appearing in eqs. 2.88 and 2.90 were specified on the basis of independent experimental results. The temperature dependence of the rate was in excellent agreement with experimental observation [113-116] for the three adsorbate-metal systems studied. The preexponential factor for adsorbed CO was found to be a factor of 100 larger than the value of $1013 \mathrm{~s}^{-1}$ for adsorbed $K$ or $X e$. This difference was attributed to the frustrated surface rotational modes of chemisorbed $\mathrm{CO}$.

\subsection{Quantum Mechanical Models}

The earliest quantum models, developed by Lennard-Jones, Strachan, and Devonshire [117-121], treated the one-dimensional motion of an adsorbate bonded to a single surface atom. The motion of the adsorbate was only considered in the direction perpendicular to the surface, and only single-phonon exchanges between the substrate and adsorbate were taken into account. As al consequence of the one-dimensional nature of the adsorbate motion, all exchange 
of energy between the adsorbate and the substrate leads to motion perpendicular to the surface, with the net result that the transition probabilities from bound to continuum states are overestimated [122].

De et al. [123] have used a one-dimensional, multiphonon model to represent the desorption of $K$ and $X_{e}$ from a $W$ surface. The phonon spectrum of the substrate was described by a Debye model, and either a harmonic or a Morse potentials was used to describe the interactions between the adsorbate and the substrate. Although the rate of desorption predicted by the model was found to be sensitive to the type of potential used in the calculations, both harmonic and Morse potentials resulted in an Arrhenius dependence of the desorption rate. Good agreement between theory and experiment [113] was obtained for the desorption rates of $K$ from a $W$ surface when the interactions between the adsorbate and the surface were described by a Morse potential. On the other hand, for the desorption of $\mathrm{Xe}$ from a $W$ surface, the predicted rates of desorption, using either a harmonic or a Morse potential, were smaller by a factor of 100 than the rates determined experimentally [115]. No explanation for this discrepancy was given by the authors.

Hood et al. [44] have used a one-dimensional quantum model with a Morse potential to analyze the desorption of Ar from a $W$ surface. Included in the model were multiphonon exchange processes between the $\mathrm{Ar}$ atoms and the substrate. The rates of desorption predicted by the model exhibited weakly non-Arrhenius behavior. The apparent preexponential factor increased from $1010 \mathrm{~s}^{-1}$ to 1011 $s^{-1}$ with increasing temperature, and the apparent activation energy 
increased from $1.3 \mathrm{kcal} / \mathrm{mol}$ to $1.5 \mathrm{kcal} / \mathrm{mol}$. These authors noted that the apparent activation energy was $25-35 \%$ lower than the binding energy of $1.9 \mathrm{kcal} / \mathrm{mol}$ used in the Morse potential. This was ascribed to the high desorption probability of adsorbates promoted to bound states of intermediate energy. As a consequence, the apparent desorption activation energy barrier is the energy necessary to reach intermediate bound states rather than that required to reach the top of the potential well.

Hood et al. [44] have also examined the desorption kinetics of $\mathrm{CO}$ from a $\mathrm{Cu}$ surface using the model discussed above. The CO molecule was treated as a quasi-atom, i.e., no account was made for the $\mathrm{CO}$ bending and stretching modes of vibration. The calculated rates of desorption were observed to exhibit strongly non-Arrhenius behavior as a function of temperature and to vary over approximately one order of magnitude depending on the mode of phonon relaxation used in the calculations. The apparent preexponential factor for desorption ranged from 1013 to $1017 \mathrm{~s}-1$, with the smaller values being observed at lower temperatures. The activation energies for desorption were seen to be $5-20 \%$ smaller than the binding energy value of $16.6 \mathrm{kcal} / \mathrm{mol}$ used in the Morse potential. This observation was explained in the same manner as that for the case of Ar desorption from W, discussed above.

\subsection{Simulation of TPD Spectra}

Both continuum lattice-gas models and Monte Carlo (stochastic) models have been used to simulate TPD spectra of 
adsorbates from well-defined surfaces. In the continuum approach, both the explicit coverage dependence of the desorption rate and the dependence of the rate coefficient, $k_{d}(\alpha)$, are described in terms of the average coverage, $\theta$. By contrast, Monte Carlo models of desorption represent the desorption process in terms of a probability which is specific to each site and its configuration of nearest neighbors. As will be shown below, the influence of finite rates of surface diffusion and the presence of coadsorbates can be incorporated into either type of model.

Simulations Based on Continuum Models

To simulate TPD spectra within the continuum framework, a balance is written between the rate of desorption and the rate at which the surface coverage decreases. Thus,

$r_{d}=-\beta \frac{d \theta}{d T}$

where $\beta=d T / d t$ is the rate at which the surface is heated. If lateral interactions between adsorbates can be neglected, $r_{d}=v_{d}(\alpha) \exp (-$ $\left.E_{d} / k_{b} T\right)^{\alpha}$. Integration of eq. 5.1 then yields $\theta(T)$, from which it is possible to determine $r d$ as a function of $T$, for an initial adsorbate coverage, $\theta_{0}$.

Experience has shown that the assumption of coverageindependent rate parameters does not adequately represent the full shape of TPD spectra observed experimentally. Substantially better agreement between theory and experiment can be achieved using a lattice-gas model of adsorbate-adsorbate interactions. Several examples of the use of lattice-gas models to simulate TPD spectra are presented below In all but one case, the distribution of 
adsorbates on the surface is based on the quasi-chemical approximation (QCA).

Goymour and King [124] have simulated the associative desorption of the high-temperature states of dissociated $C D$ from a tungsten ribbon. The adsorbed $C$ and $O$ atoms were assumed to have equivalent binding energies and the activation energy for associative desorption was set equal to the differential heat of adsorption. The values of the preexponential factor $\left(v_{d}(2)=3 \times 1014 s^{-1}\right)$, the activation energy at low coverage $\left(E_{d^{\circ}}=100 \mathrm{kcal} / \mathrm{mol}\right)$, and the nearest-neighbor interaction energies $(w c O=w O O=w c c=4.8$ $\mathrm{kcal} / \mathrm{mol}$ ) were determined by fitting the TPD spectrum predicted by the model to the experimentally observed TPD spectrum. As illustrated in figs. $5.3 \mathrm{a}$ and $5.3 \mathrm{~b}$, the predicted CO TPD spectrum agrees with that observed experimentally [125]. The coverage dependence of the activation energy for CO desorption was also determined fram the model and, as seen in fig. 5.3c, decreases with increasing $\mathrm{CO}$ coverage as a consequence of the repulsive $\mathrm{C}-\mathrm{O}, \mathrm{O}-\mathrm{O}$, and $\mathrm{C}-\mathrm{C}$ interactions.

Zhdanov [126] has also evaluated the applicability of a latticegas model for describing desorption from well-defined metal surfaces. Figure 5.4 shows a comparison of the theoretical and experimental [127] TPD spectra for CO desorption from $\operatorname{Ir}(110)$. The shape and location of the predicted spectrum is in fair agreement with the experimental results. To obtain this level of agreement, the following parameters were used: $E_{d^{\circ}}=33 \mathrm{kcal} / \mathrm{mol}$, wco $\cdot c 0=2.2$ $\mathrm{kcal} / \mathrm{mol}$, and $v_{d}(1)=1011 \mathrm{~s} \cdot 1$. It should be noted that the value of $v_{d}(1)$ used to fit the data is considerably smaller than values 
reported ir: the experimental literature, which range from 1014 $1016 \mathrm{~s}-1$.

A second example from the work of Zhdanov [126] is shown in fig. 5.5. In this instance, the TPD spectra are for the associative desorption of $\mathrm{O}_{2}$ from an $\operatorname{Ir}(110)$ surface. The parameters $E_{d^{\circ}}=67$ $\mathrm{kcal} / \mathrm{mol}$, woo $=3.6 \mathrm{kcal} / \mathrm{mol}$, and $v_{d}(2)=3.5 \times 1011 \mathrm{~s}^{-1}(3.5 \times 10.4$ $\mathrm{cm}^{2} / \mathrm{s}$ ) were used to represent the data, and reasonable agreement between the theoretical and experimental [128] results is observed. The activation energy for $\mathrm{O}_{2}$ desorption determined from the model was seen to decrease as a function of increasing coverage as a consequence of the repulsive $0-0$ interactions.

While most applications of lattice-gas models have been restricted to a rigid lattice of adsorption sites, Zhdanov [60] has demonstrated the extension of this model to cases where surface reconstruction occurs. The rate of desorption was written in terms of a chemical potential which included contributions from adsorbateadsorbate interactions, adsorbate-surface interactions, and the surface-free energy. Illustrations of the variations of $\Delta E_{d}(\theta)$ and $v_{d}(1)(\theta) / v_{d}(1)(0)$ determined from the model are shown in fig 5.6. Both the preexponential factor and the activation energy are seen to decrease with increasing coverage. The variation in the apparent preexponential factor over 8 orders of magnitude is in accord with what has been determined for the associative desorption of $\mathrm{H}_{2}$ from a W(110) surface [129]. More recently, Myshlyavtsev and Zhdanov [130] have reexamined the the role of surface reconstruction with a more accurate approach. In this study, the variation of the preexponential factor with coverage was much smaller, spanning 
only two orders of magnitude.

Implicit in the QCA is the assumption that surface diffusion is much more rapid than desorption so that an equilibrium configuration of adsorbates is maintained at all times. Sundaresan and Kaza [131] have investigated the effects of limited adspecies mobility on the rates of desorption when two species are coadsorbed on a surface. A lattice-gas model was used to describe the effects of lateral interactions. To account for the mobility of the adsorbate, a set of three differential equations was used to describe the change in the occupation probabilities of pairs of sites with time. It was demonstrated that the TPD spectra for coadsorbed species are sensitive to the sequence in which the adsorbates are dosed on the surface as well as to the relative coverages and mobilities of the two species, but no direct comparison was made between theory and experiment.

Simulations Based on Monte Carlo Models

In the Monte Carlo approach for simulating TPD spectra, adsorbates are assumed to occupy well-defined sites on a fixed lattice. The occupancy of a given site can change as a consequence of either desorption or hopping (diffusion) of the adsorbate to an adjacent site. The probability of desorption from site $i$ is defined by $P_{i}$ and the probability for diffusion from site $i$ to site $j$ is defined by $P_{i j}$.

The probability of desorption of a given species from site $i$ in the time interval $\Delta t$ can be defined as

$P_{i}=v_{d, i} \exp \left(-E_{d, i} / k_{b} T\right) \Delta t$

where $v_{d, i}$ and $E_{d, i}$ are the preexponential factor and the activation 
energy for the local environnient i, respectively. The value of $\Delta t$ in eq. 5.2 is chosen so that $P_{i}$ goes to unity at a temperature sufficiently high to guarantee virtually complete desorption from all sites of type $i$.

The rate of desorption, $\mathrm{rd}$, expressed as a turnover frequency based on the number of surface atoms, $N_{s}$, is given by $r_{d}=\frac{N_{d}}{\alpha N_{s} \Delta t}$

where $\mathrm{No}_{\mathrm{d}}$ is the number of adsorbates atoms or molecules desorbing in the time interval $\Delta t$. The parameter $\alpha$ is 1 for atomic or molecular desorption and 2 for associative desorption. Consequently, rd is the rate of desorption as observed from the gas phase.

The diffusion of adsorbates can be handled using the Monte Carlo techniques described in Section 4.4. If the rate of diffusion is of comparable magnitude to the rate of desorption, then the dynamics of diffusion are represented with an energy barrier model and $P_{i j}$ is given by

$P_{i j}=\exp \left[-E_{\text {diff }} / k_{b} T\right]$

If diffusion occurs very rapidly relative to diffusion, the spatial distribution of adsorbates on the surface will remain very close to equilibrium. Under such circumstances, the probability of moving an adsorbate can be represented by Kawasaki dynamics:

$P_{i j}=\frac{\exp \left[-\left(Q_{i}-Q_{j}\right) / k_{b} T\right]}{1+\exp \left[-\left(Q_{i}-Q_{j}\right) / k_{b} T\right]}$

where $Q_{i}$ and $Q_{j}$ are the heats of adsorption for an adsorbate at sites $i$ and j, respectively. Application of eq. 5.5 for a large number of 
hops leads to an equilibrium distribution of adsorbates on surface. The probability and rate formulations described above are incorporated into a Monte Carlo algorithm for simulating the temperature-programmed desorption of adsorbates. The algorithm presented below is for simulations in which adsorbate diffusion is described by Kawasaki dynamics, the case most frequently treated in the literature.

The surface is represented by an array of numbered sites, and periodic boundary conditions are used to eliminate edge effects. Adsorbate atoms or molecules are placed on the surface lattice to achieve a desired initial coverage, $\theta_{0}$. The temperature is initialized at To and taken to be constant at this value for the time interval $\Delta t$. During this time interval, the desorption of adsorbates from occupied sites is determined by the outcome of a comparison between a random number, $R$, and the probability condition for desorption $P_{i}$. When $R \leq P_{i}$, the adsorbate (or pair of adsorbates for associative desorption) is removed from the lattice, and $N_{d}$ is incremented by one (two for associative desorption). If, on the other hand, $R>P_{i}$, the adsorbate remains on the lattice. After sampling of the surface is completed, the rate of desorption for this time interval is calculated from eq. 5.3.

Following the calculation of the rate of desorption, the remaining adsorbates are redistributed on the lattice to account for the effects of surface diffusion. Redistribution of the adsorbates to achieve a new equilibrium configuration is achieved as follows. Movement of an adsorbate at site $i$ to a vacant site $j$ is determined by comparing a random number $R$ with the value of $P_{i j}$ given by eq. 
5.5. If $R \leq P_{i j}$, the adsorbate is moved from site $i$ to site $j$, whereas if $R>P_{i j}$, the adsorbate is not moved. Application of this test to the ensemble of adsorbates for a sufficiently large number of hops leads to a minimization of the surface energy and a reestablishment of equilibrium.

The desorption/diffusion calculations described above constitute a Monte Carlo Step (MCS). As noted above, the time interval associated with an MCS is $\Delta t$, and over this interval the temperature is constant. At the end of an MCS, the temperature is increased by the increment $\beta \Delta t$, where $\beta$ is the heating rate. A new MCS is then carried out at the next temperature. This process is repeated until a temperature is reashed for which the surface is depleted of adsorbate. A plot of the desorption rate as a function of temperature then yields a TPD spectium. Several examples of the simulation of TPD spectra using Monie Carlo methods are presented below.

In the absence of lateral interactions, Lombardo and Bell [132] have demonstrated that the continuum and stochastic representations of TPD lead to identical spectra. An illustration of this point for non-associative desorption is given in fig. 5.7. The slight deviations of the Monte Carlo simulations from the curve for the continuum model are attributable to the combined effects of finite lattice size, finite step size, and statistical, random-number fluctuations.

Sales and Zgrablich [133] have utilized a Monte Carlo approach to model the influence of lateral interactions between adsorbates on TPD spectra. Pairwise-additive interactions were used to account 
for the lateral interactions between adsorbates. Figure $5.8 \mathrm{a}$ shows the simulated TPD spectra for non-associative desorption from a onedimensional surface for selected values of the interaction parameter WAA. The $p$ edicted TPD spectra based on a continuum lattice-gas model using the Bragg-Williams approximation $\left(E_{d}=E_{d}{ }^{\circ}-\right.$ $Z W A A \theta$, where $z$ is the number of nearest-neighbor sites around a given site) [134] are presented in fig. 5.8b. A comparison of the two figures demonstrates that, for $W A A=0$ (no interactions) and for WAA < 0 (attractive interactions), the TPD spectra determined by the Monte Carlo and contiriuum lattice-gas models are in good-agreement. For WAA > 0 (repulsive interactions), however, the Monte Carlo model predicts two peaks whereas the continuum lattice-gas model predicts one. The authors indicate that the difference between the two models arises from the incorrect assumption of a random distribution of adsorbates in the Bragg-Williams approximation. The influence of the number and distribution of surface binding sites with different energies on TPD spectra was also examined by Monte Carlo simulations, and it was shown that the shape and location of simulated TPD spectra are sensitive to the distribution of adsorbates on the surface as well as to the magnitude and sign of the energetic interactions between species.

In another study, Sales et al. [135] have compared the desorption kinetics predicted from a Monte Carlo model using pairwise-additive energetics for nearest- and next-nearest neighbor interactions with the desorption kinetics predicted from a continuum lattice-gas model using the quasi-chemical approximation for nearest-neighbor interactions and the Bragg- 
Williams approximation for next-nearest neighbor interactions. As seen in fig. 5.9, the two models predict many of the same features for selected values of the lateral interaction parameters. The number of peaks, the peak locations, and the coverage dependence of the peak positions predicted by the two models, however, are different. The differences in the spectra predicted from the two models are especially pronounced for the simulations incorporating repulsive nearest-neighbor interactions with attractive nextnearest neighbor interactions.

Hood et al. [61] have combined Monte Carlo simulations and continuum rate expressions to describe molecular desorption of $\mathrm{N}_{2}$ from a Ru(001) surface. The Monte Carlo segment of the algorithm was used to determine the local environment of each adsorbate and hence the local activation ennrgy for desorption. The coverage and activation energy for each type of surface environment were then used in a continuum rate expression such as eq. 5.1 to solve for the rate of desorption. The parameters used to simulate the TPD spectra were repulsive nearest-neighbor interactions of $0.25 \mathrm{kcal} / \mathrm{mol}$, attractive next-nearest neighbor interactions of $0.45 \mathrm{kcal} / \mathrm{mol}$, and a preexponential factor of $1012 \mathrm{~s}-1$. In addition, the preexponential factor was postulated to have an increasing exponential dependence with increasing global surface coverage of $\mathrm{N}_{2}$. As illustrated by figs. $5.10 \mathrm{a}$ and $5.10 \mathrm{~b}$, the simulated TPD spectra agree with experimental observation [62,63]. The low-coverage peak was attributed to desorption of $\mathrm{N}_{2}$ molecules located at the perimeter of $\mathrm{N}_{2}$ islands in which the molecules were arranged in $(\sqrt{3} \times \sqrt{3}) \mathrm{R} 30^{\circ}$ domains, whereas the high-coverage peak was attributed to 
desorption of $\mathrm{N}_{2}$ from antiphase $(\sqrt{3} \times \sqrt{3}) \mathrm{R} 30^{\circ}$ domains located within the $\mathrm{N}_{2}$ islands.

Lombardo and Bell [132] have developed a Monte Carlo algorithm in which the BOC-MP method $[15,16]$ was used to calculate the activation energy for desorption. For non-associative desorption, $E_{d}$ is given by

$E_{d}=\dot{Q}_{A, n^{\prime}}$

and for associative desorption of $A_{2}, E_{d}$ is given by

$E_{d}=\dot{Q}_{A^{\prime}, n^{\prime}} \dot{Q}_{A^{\prime}, n^{\prime}} /\left(\dot{Q}_{A^{\prime}, n^{\prime}}+\dot{Q}_{A^{\prime}, n^{\prime}}\right)$

where $Q_{A, n^{*}}$ and $Q_{A^{\prime}, n^{*}}$ represent the heats of adsorption of $A$ and $A^{\prime}$, respectively. The two recombining atoms are designated $A$ and $A^{\prime}$ to denote that the local environments of each may be different. In order to account for coverage effects, $Q_{A, n^{*}}$ and $Q_{A^{*}, n^{*}}$ are determined from eq. 2.8 .

When metal-adsorbate and adsorbate-adsorbate interactions between adsorbates were included, Lombardo and Bell [132] observed multiple peaks in the simulated TPD spectra, and the activation energy profile of the desorbing species was found to decrease in a non-linear fashion with increasing coverage. It was also shown that the activation energy of the desorbing species is less than or equal to the average value for the entire adlayer. Physically, this means that for a given coverage, adsorbates which are in less stable configurations have a lower activation energy for desorption and hence desorb preferentially.

In the same study, Lombardo and Bell [132] reported simulations of the associative desorption of $\mathrm{H}_{2}$ from $\mathrm{Mo}(100)$. As 
illustrated by figs. 5.11a and 5.11b, the simulated TPD spectra for $\mathrm{H}_{2}$ desorption from a $\mathrm{Mo}(100)$ surface agree with the experimentally observed spectra [136]. Figure 5.12a and 5.12b shows a comparison of the simulated and experimentally observed [137] profiles of the activation energy as a function of coverage. Both plots exhibit a step-wise decrease in activation energy as the coverage increases. The observed decrease in the activation energy with increasing coverage is caused by a decrease in the heat of adsorption of $\mathrm{H}$ atoms when more than one atom is bonded to a given metal atom.

Monte Carlo models have also been used to simulate the desorption of coadsorbed species. Gupta and Hirtzel [138] have investigated the effects of lateral interactions on liie nonassociative desorption of coadsorbed species A and B. Both nearestand next-nearest neighbor interactions between species were represented in a pairwise additive fashion. It was shown that the number of peaks in the TPD spectrum and the spectrum shape and location are sensitive to the lateral interactions and to the relative coverage of each species. These authors also demonstrated that A-B interactions only affect the spectrum of the species desorbing at lower temperatures whereas $A-A$ and $B-B$ interactions only affect the spectra of the respective $A$ and $B$ species.

More recently, Lombardo and Bell [139] have simulated TPD spectra for coadsorbed species using the BOC-MP approach to describe the effects of adsorbate coverage on the energetics of desorption. Nearest-neighbor interactions between the adsorbates and the metal surface as well as nearest-neighbor interactions between the coadsorbates were taken into account. The presence of 
a strongly bound coadsorbate on a $b c c(100)$ surface was shown to shift the associative desorption spectrum of adsorbed atoms to lower temperatures. The simulated TPD spectra were found to be in qualitative agreement with experimental results for $\mathrm{H}_{2}$ coadsorbed with strongly bound atomic species on $\mathrm{Mo}(100)$ [140] and $\mathrm{Fe}(100)$ [141] surfaces.

TPD spectra were also simulated for the concurrent desorption of $B$ molecules and the associative desorption of $A$ atoms from an fcc(100) surface [139]. Two types of behavior were observed: in one case, both species exhibited new low-temperature features not present in the TPD spectra of $A$ and $B$ when each species was adsorbed alone. In the second case, only the more weakly bound species displayed new spectral features. These types of behavior are in qualitative agreement with what has been observed for the codesorption of $\mathrm{CO}$ and $\mathrm{H}_{2}$ from $\mathrm{Ni}(100)$ [142] and $\mathrm{Rh}(100)$ [143] surfaces, respectively. Figure $5.13 a$ shows TPD spectra for $A_{2}$ and $B$ when each species is adsorbed separately, and when both species are coadsorbed. The experimentally observed TPD spectra for $\mathrm{CO}$ and $\mathrm{H}_{2}$ on a $\mathrm{Ni}(100)$ are shown in fig. 5.13b [142]. A comparison of the two figures demonstrates the qualitative agreement between the model predictions and the experiinental observations. In fig 5.14 are shown the activation energy profiles versus coverage for both the pure component and codesorption simulations. For the $A_{2}$ and $B$ activation energy profiles when both species are coadsorbed, the activation energy profiles are lower than for the respective pure component activation energies and show larger variation with coverage. 


\subsection{Compensation Effect}

Analysis of experimental TPD spectra using empirical rate expressions of the form

$$
r_{d}=v_{d}^{(\alpha)}(\theta) \exp \left[-E_{d}(\theta) / k_{b} T\right] \theta^{\alpha}
$$

have shown evidence of a compensation effect, namely that $v_{d}(\alpha)(\theta)$ and $E_{d}(\theta)$ vary in the same manner for changes in coverage and temperature [144-149]. In particular, it has often been observed that both $v_{d}(\alpha)(\theta)$ and $E_{d}(\theta)$ decrease with increasing adsorbate coverage. While the decrease in $E_{d}(\theta)$ is readily attributable to repulsive lateral interactions, the large. decreases in $v_{d}(\alpha)(\theta)$ (e.g., up to nine orders in magnitude) have not been explained satisfactorily. Seebauer et al. [146] have recently reviewed different theoretical representations for the preexponential factor. Although several of the theories predict compensation behavior, none are able to account for the large variations such as those determined from the analysis of experimental data. The authors suggested, however, that models which account for the phonon vibrational modes of the solid and for surface reconstruction may be able to account for the large variations observed in the preexponential factor as a function of coverage.

The apparent inconsistency between the large variations in $v_{d}(\alpha)(\theta)$ deduced from experimental data and the significantly smaller variations suggested by various physical models can be reconciled in the following manner. To begin with, it must be recognized that eq. 
5.8 is written intuitively as a generalization of eqs. 2.25 and 2.26 rather than on the basis of a physical theory of desorption. For example, reference to eqs. $2.48-2.51$ shows that the rate of desorption cannot be factored naturally into a preexponential factor, an exponential factor, and a factor describing thy coverage dependence. This indicates that eq. 5.8 is not a valid physical representation of the kinetics of desorption. As a consequence, while eq. 5.8 can be fitted to experimental desorption data, the values of $v_{d}(\alpha)(\theta)$ and $E_{d}(\theta)$ determined by this means must be regarded as apparent values. Consistent with this interpretation, fig. 2.5 shows that the apparent preexponential factor deduced from a lattice-gas model in the quasi-chemical approximation exhibits a strong dependence on $\theta$, even though the proexponential factor incorporated into the model is, in fact, assumed to be constant. Inspection of eqs. 2.48-2.51 reveals further that the observed coverage dependence of the apparent preexponential factor can be ascribed to $f_{0}^{\prime}\left(\theta, W_{A A}\right)$ and $f_{0}{ }^{\prime \prime}\left(\theta, W_{A A}\right)$ which are each comprised of weighted terms of the probabilities of finding an adsorbate in a specific configuration of nearest neighbors. Viewed in this fashion, the large variations in the apparent preexponential factor can be attributed to the configurational and energetic effects of nearestneighbor interactions, rather than to an explicit dependence of the preexponential factor on coverage. 


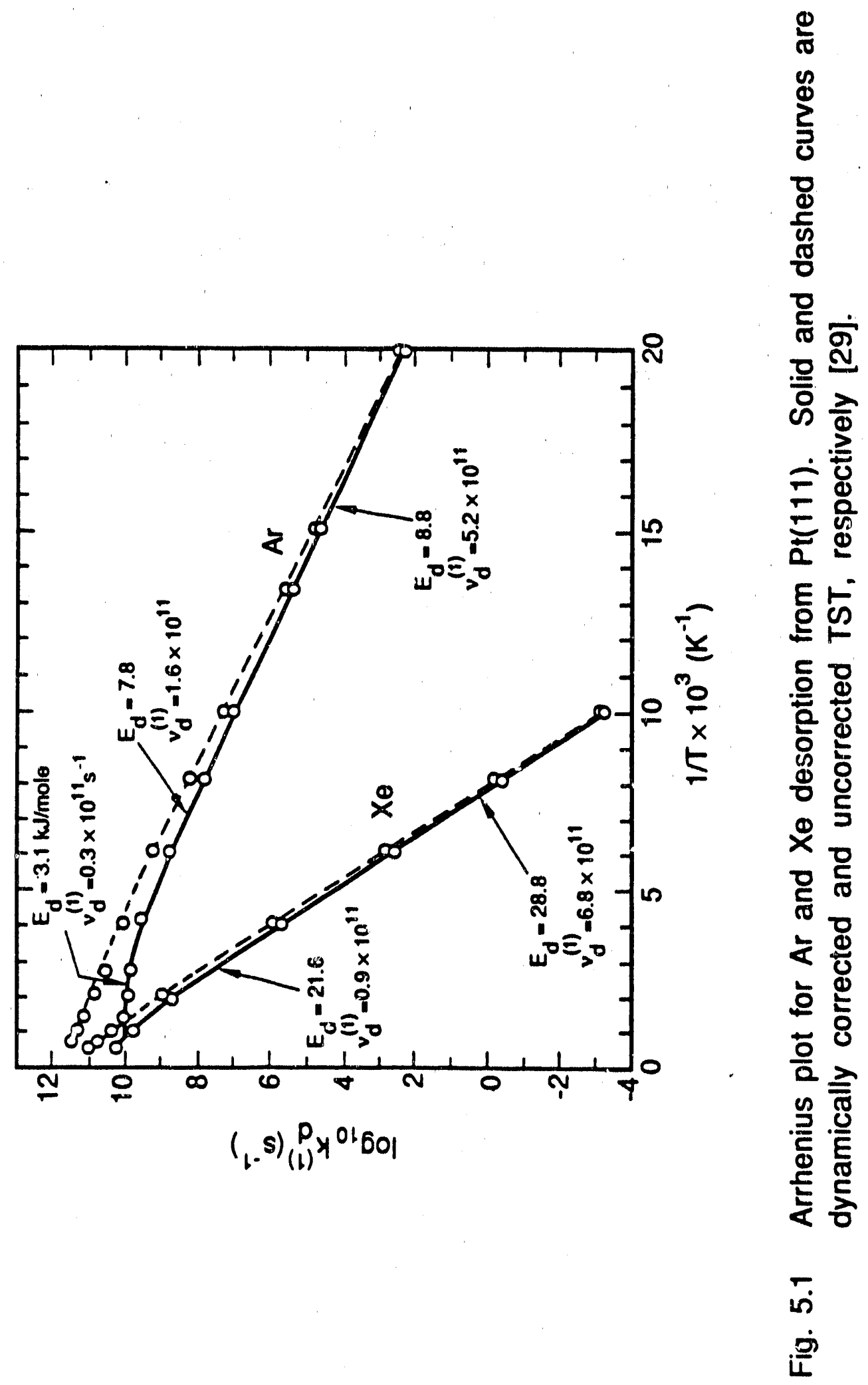




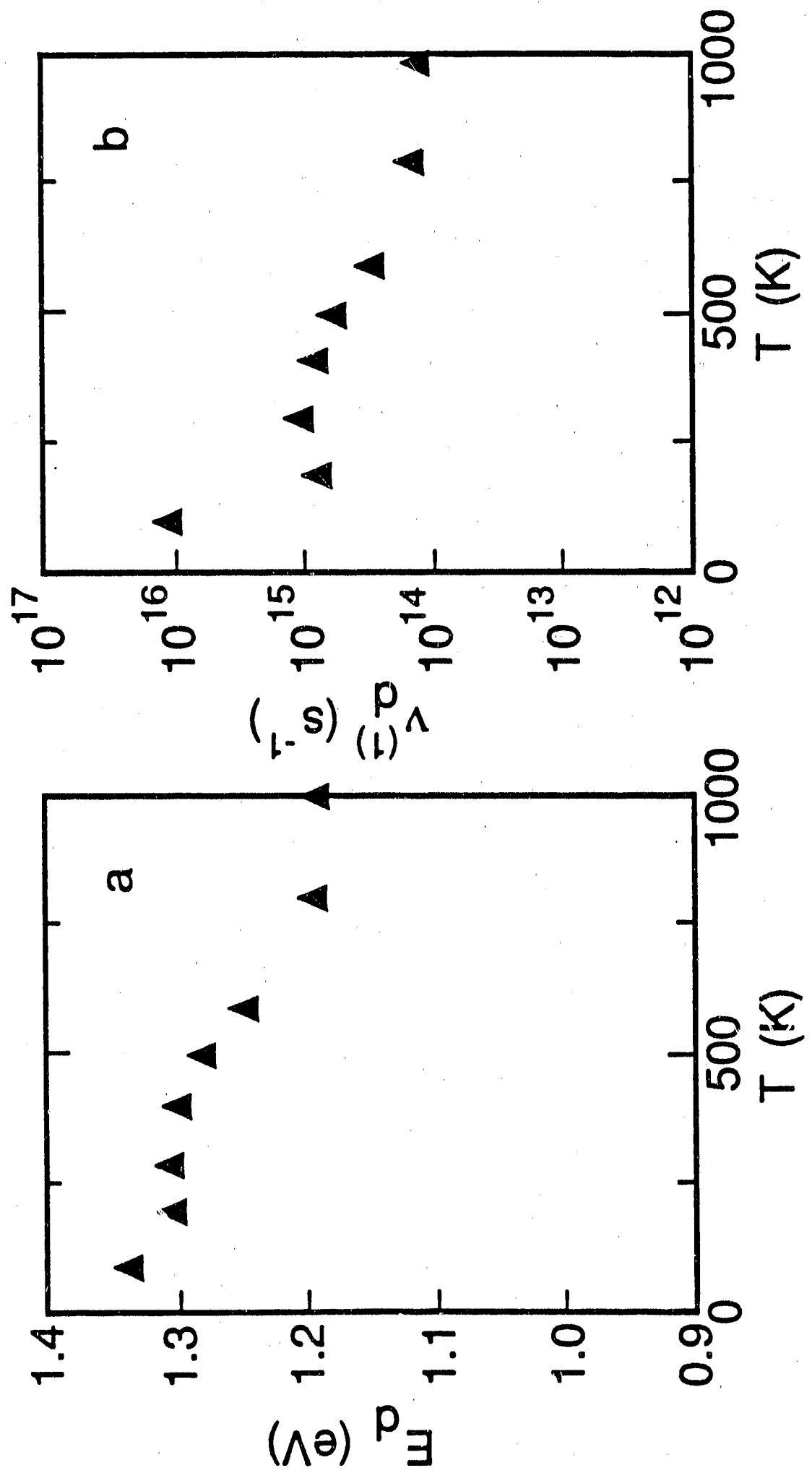

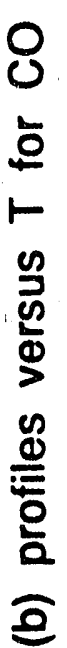

홍

들

홓토

등을

พิ

운 

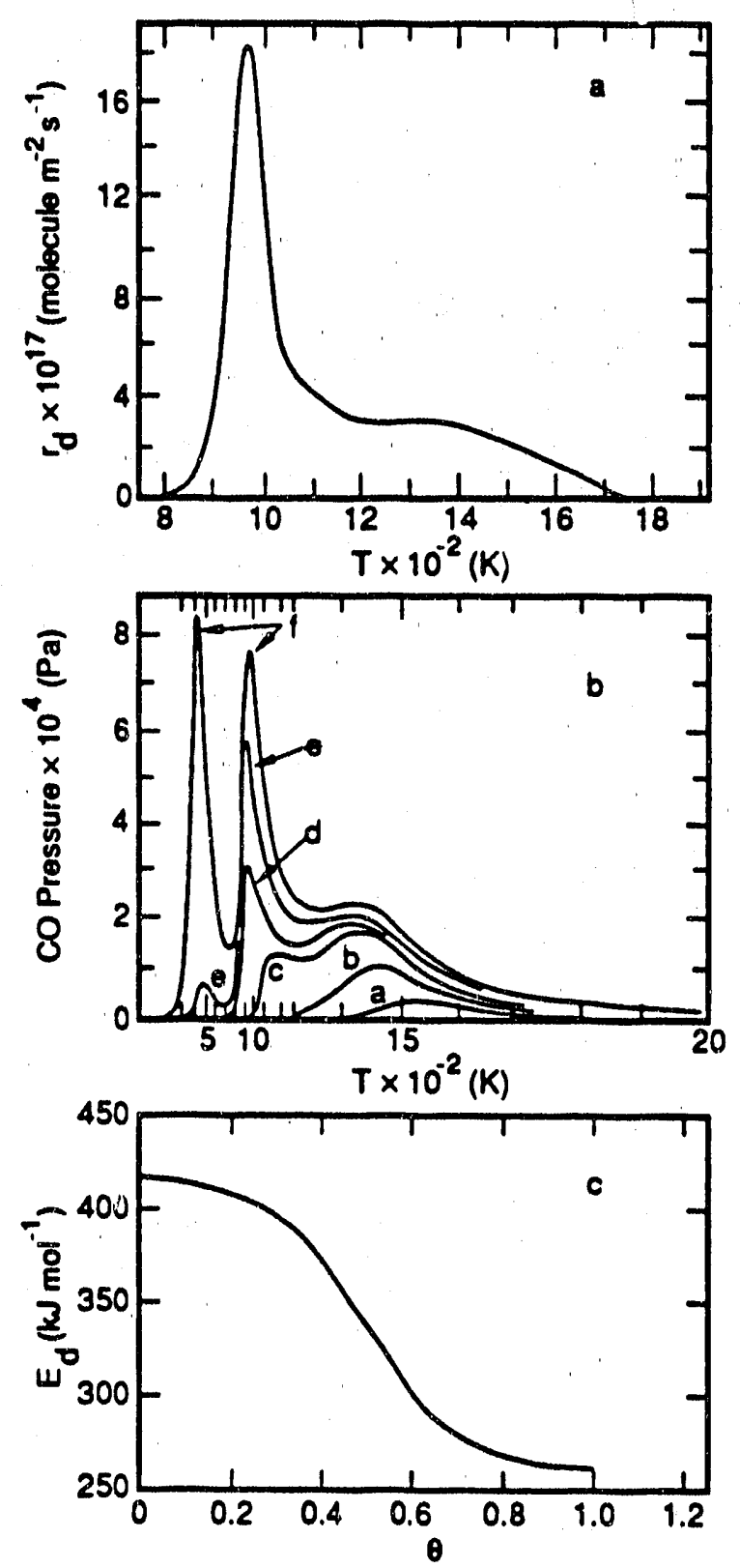

Fig. 5.3 a. Predicted CO TPD spectra from a $W$ ribbbon [124]. b. Experimental $C O$ TPD spectra from a $W$ ribbbon [125]. The $\mathrm{CO}$ exposures ( $\mathrm{Pa} \mathrm{s}$ ) are: a) $1.9 \times 10.5$; b) $6.0 \times 10.5$; c) $1.6 \times 10.4$; d) $1.9 \times 10.4$; e) $2.5 \times 10.4$; and f) $3.1 \times 10.3$. c. Predicted activation energy profile versus coverage [124]. 


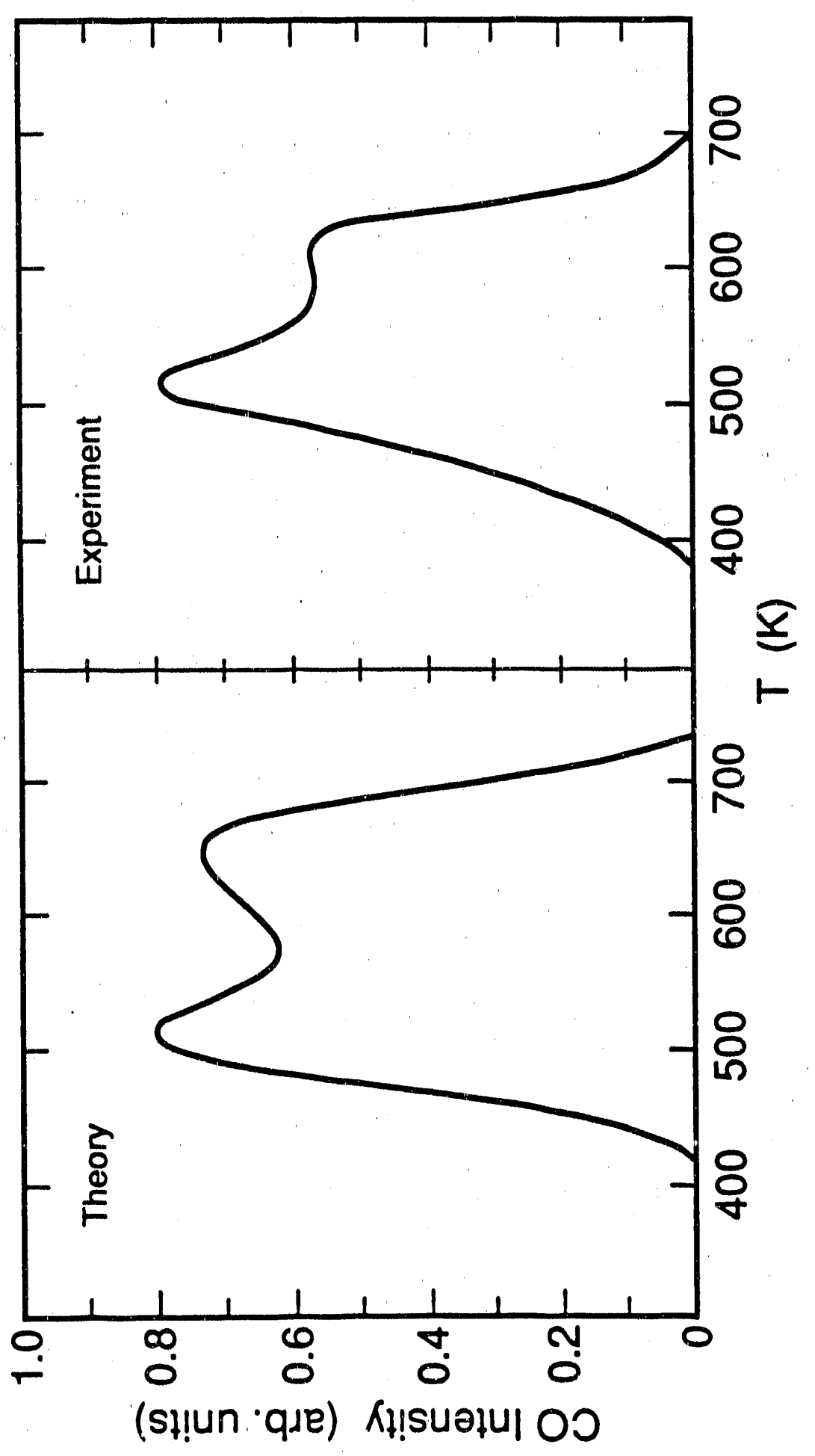

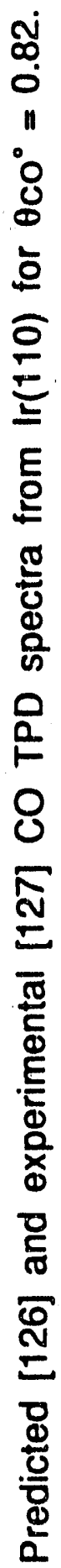

نे

iํํㄴ 


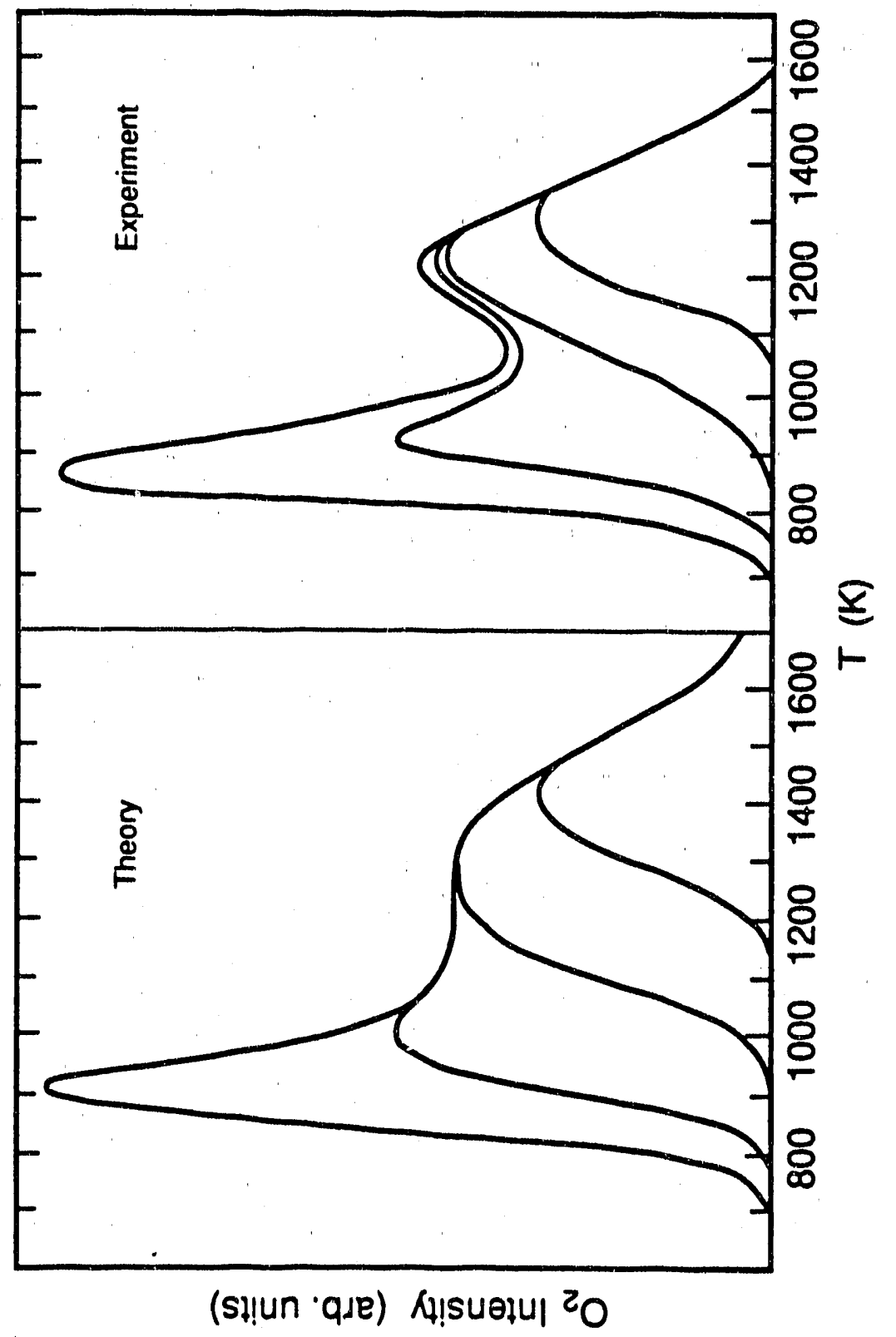

동

.

$\sum_{3}^{8}$

ㅇํำ

능

틍 은

¿

등

"

的

00

웅

ชั ธั

ธ్య

요

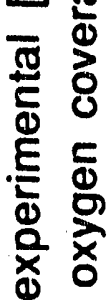

읃 오

ธ

엉

응

흔 $\frac{\omega}{\Phi}$

חึ?

iํㅛ 


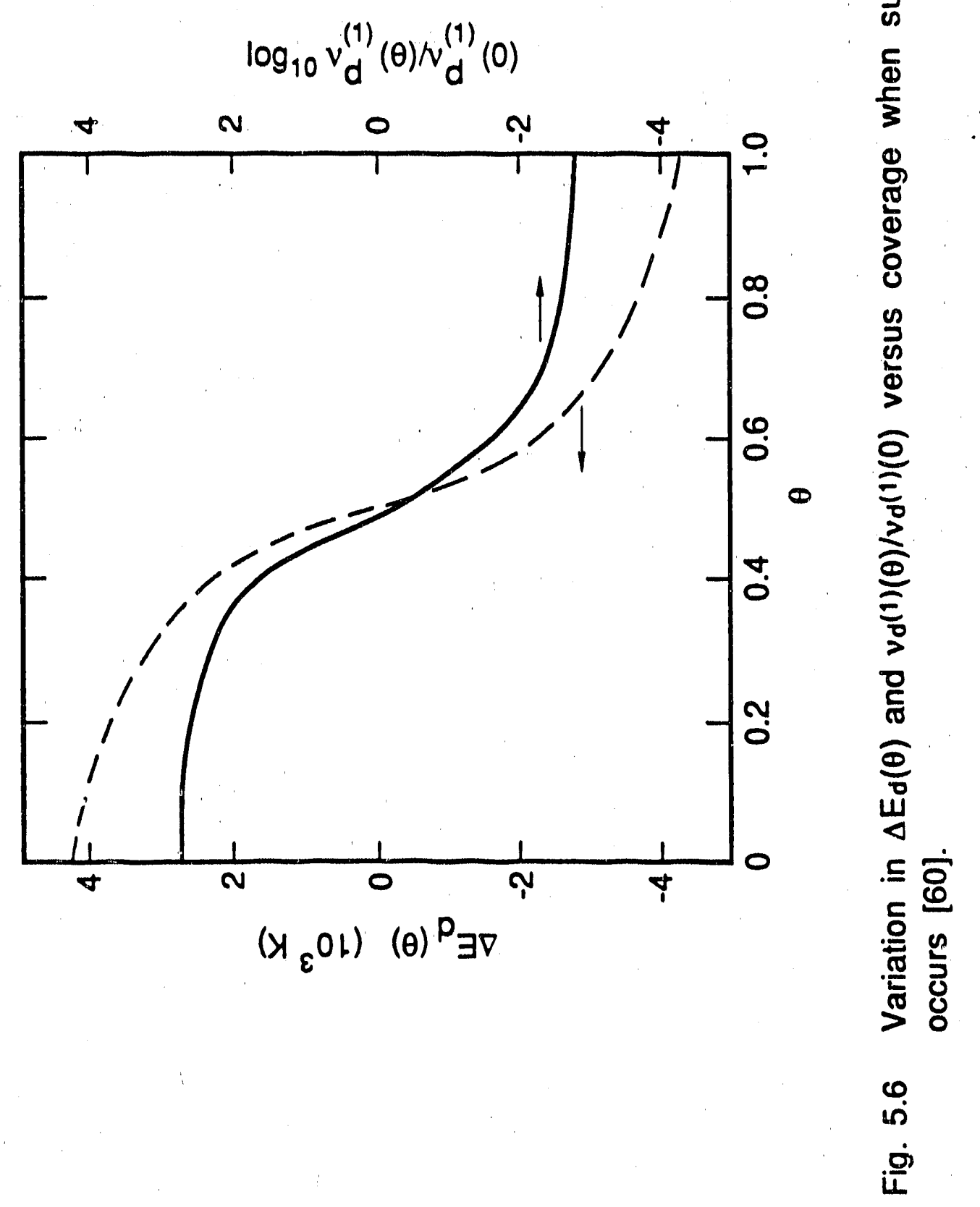




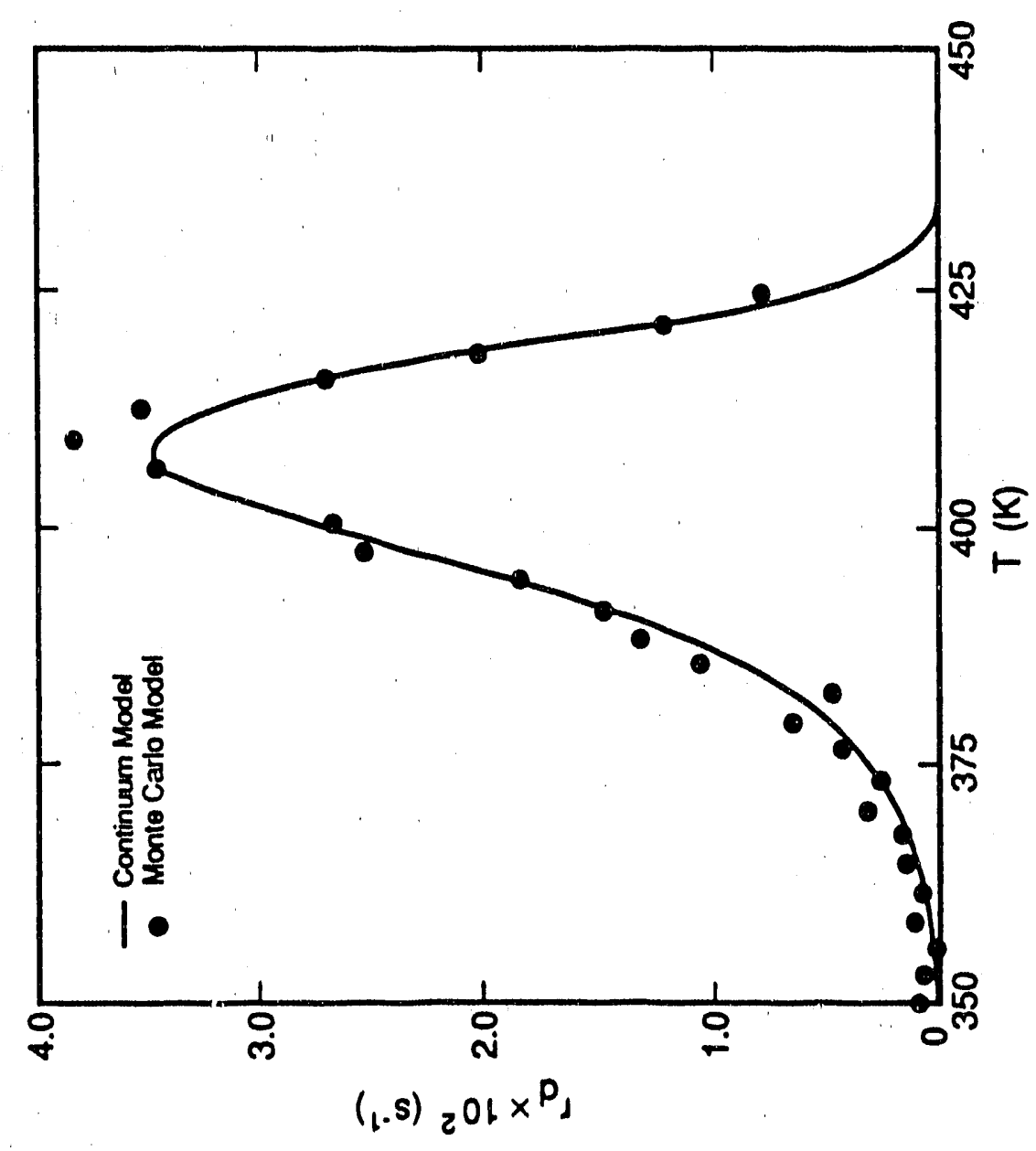

동

들 लำ

호

है

원

으.

으 $\overline{10}$

힝

응

Eั

엉

它

은 드

ญั

등 के

는

도

ำ

중

ह

ऽ ผ

م

i 

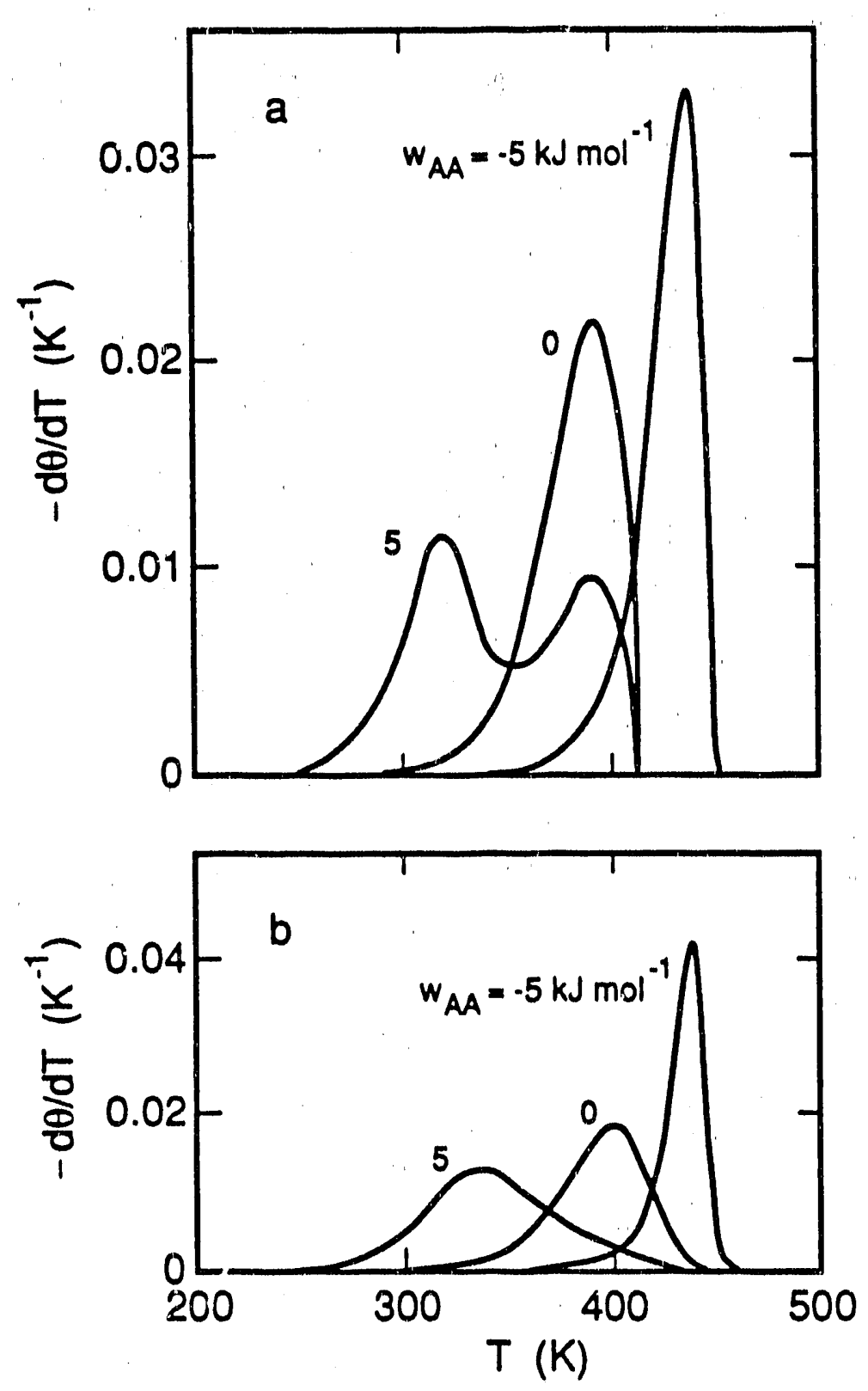

Fig. 5.8 a. Monte Carlo simulation of TPD spectra [133]. b. Continuum simulation of TPD spectra [134]. Lateral interactions between adsorbates in the continuum simulations are described with the Braggs-Willaims approximation. 


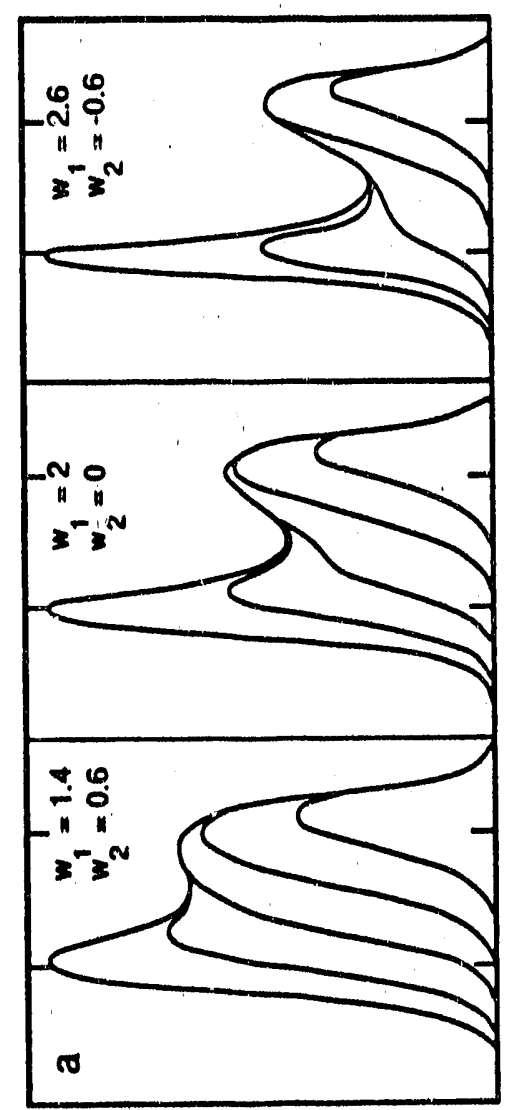

(s!!un que) ki!suoju!

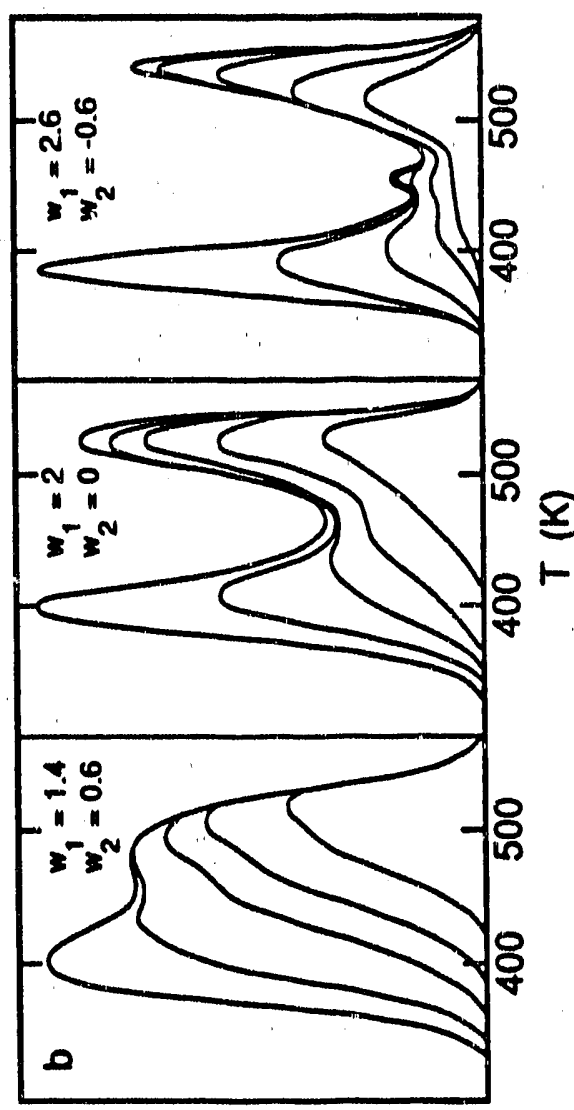

(s!!un que) Ru!suejul $\stackrel{1}{x}$

을

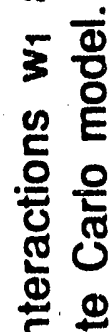

드 동

ف음

홍

$\frac{5}{\overline{1}} \overline{0}$

$\Phi$

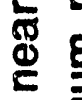

동.

ठ

응

(⿻)

冚 宊

的

i 등. 드

은

ธ.

ก 능으

กำ

二흥

동 ․․․

离

के
in 


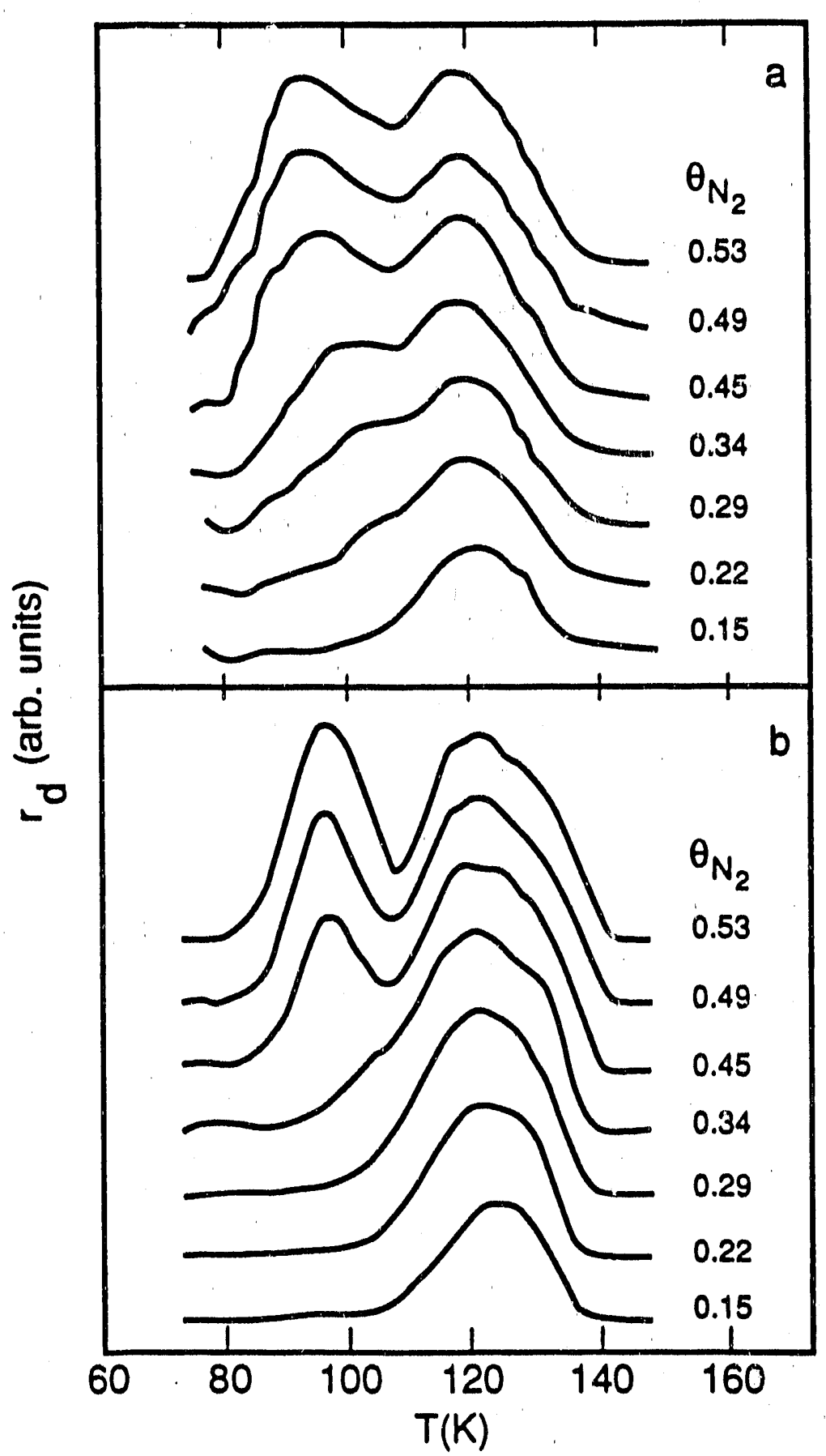

Fig. 5.10 Experimental (a) [62,63] and simulated (b) [61] TPD spectra of molecular $\mathrm{N}_{2}$ from $\mathrm{Ru}(001)$. 
116
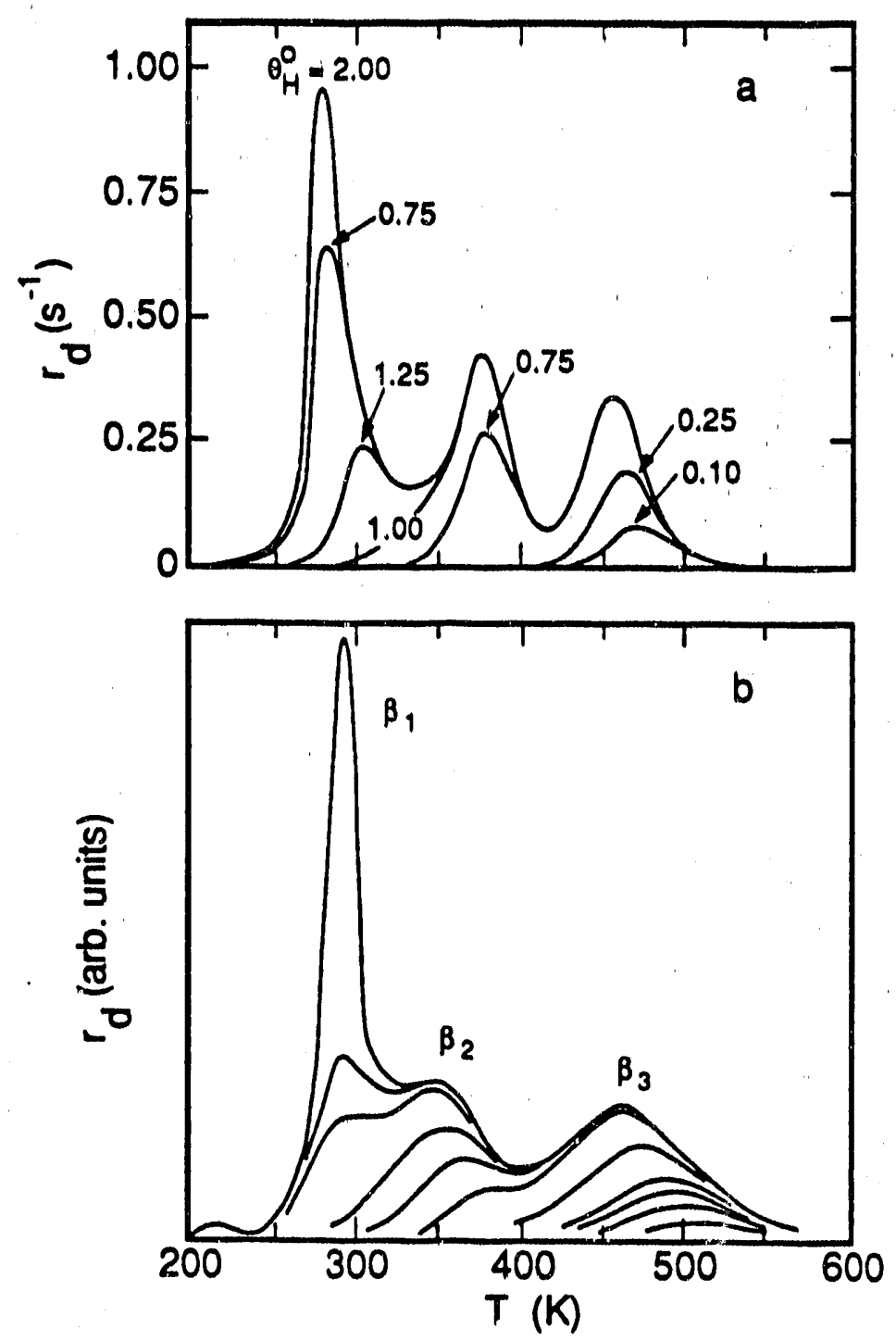

Fig. 5.11 Simulated (a) [132] and experimental (b) [136] TPD spectra of $\mathrm{H}_{2}$ from $\mathrm{Mo}(100)$. 

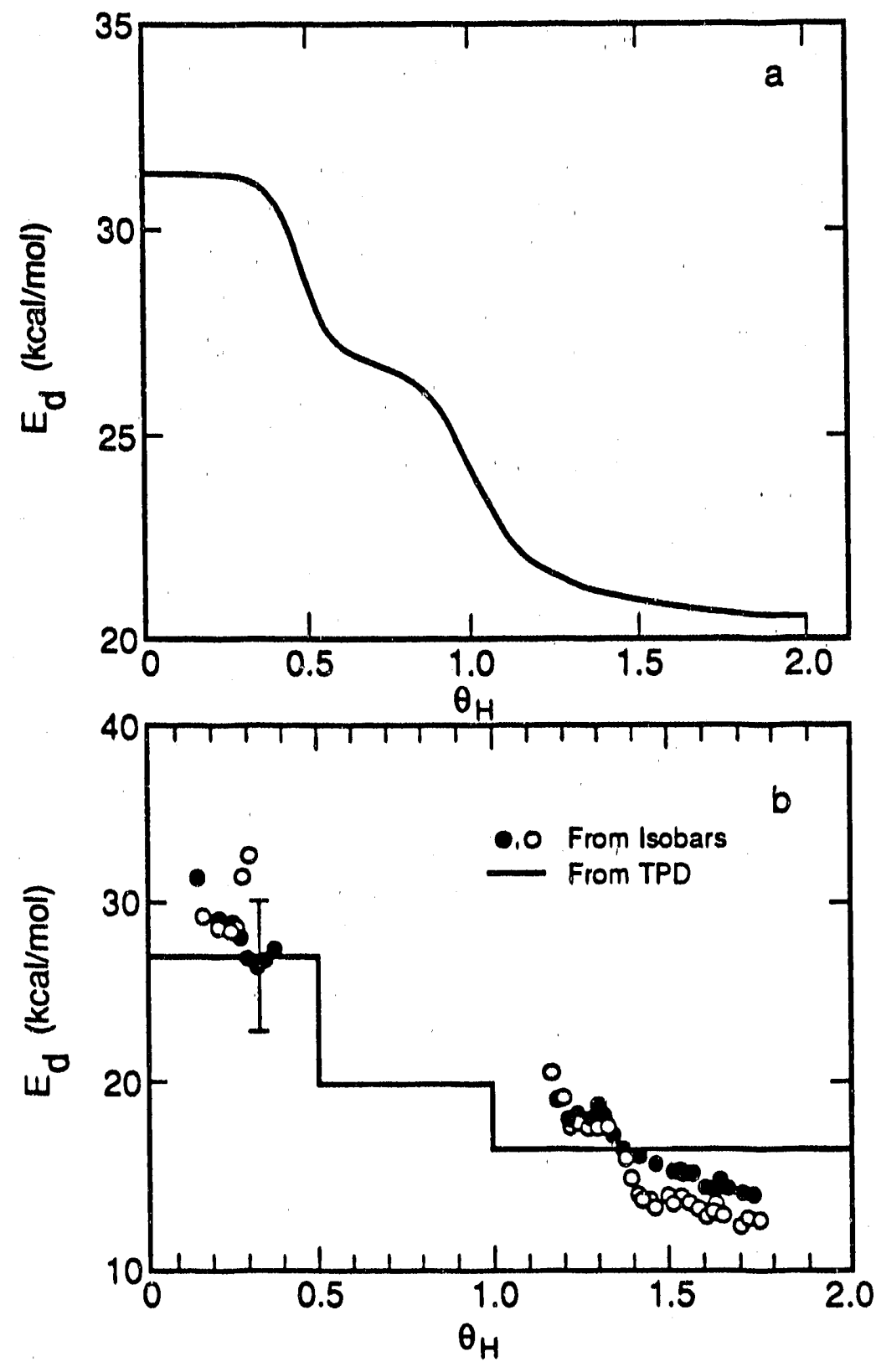

Fig. 5.12 Simulated (a) [132] and experimental (b) [137] variation in $E_{d}$ with $\theta_{H}$ for $\mathrm{H}_{2}$ desorption from $\mathrm{Mo}(100)$. 

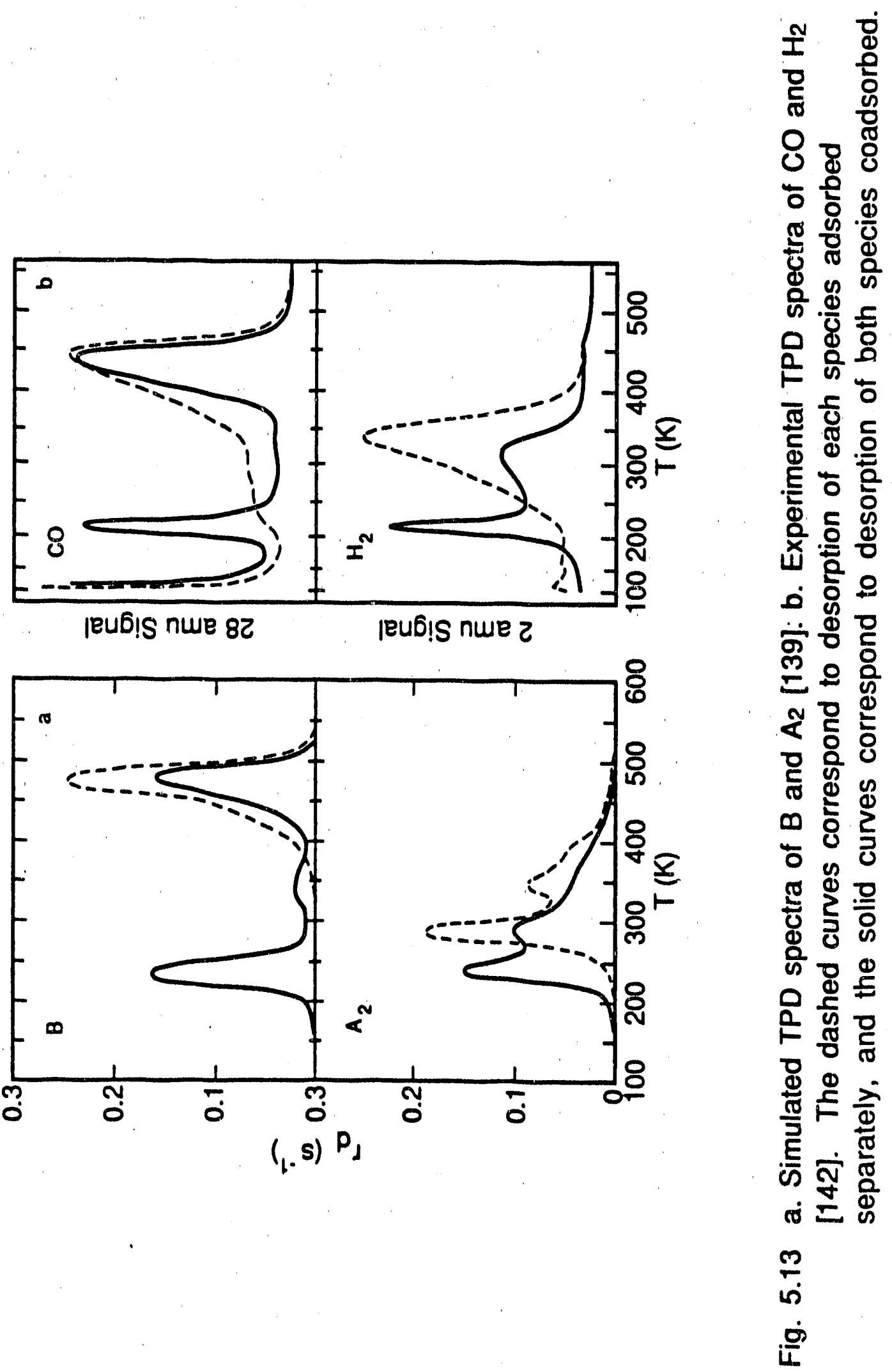


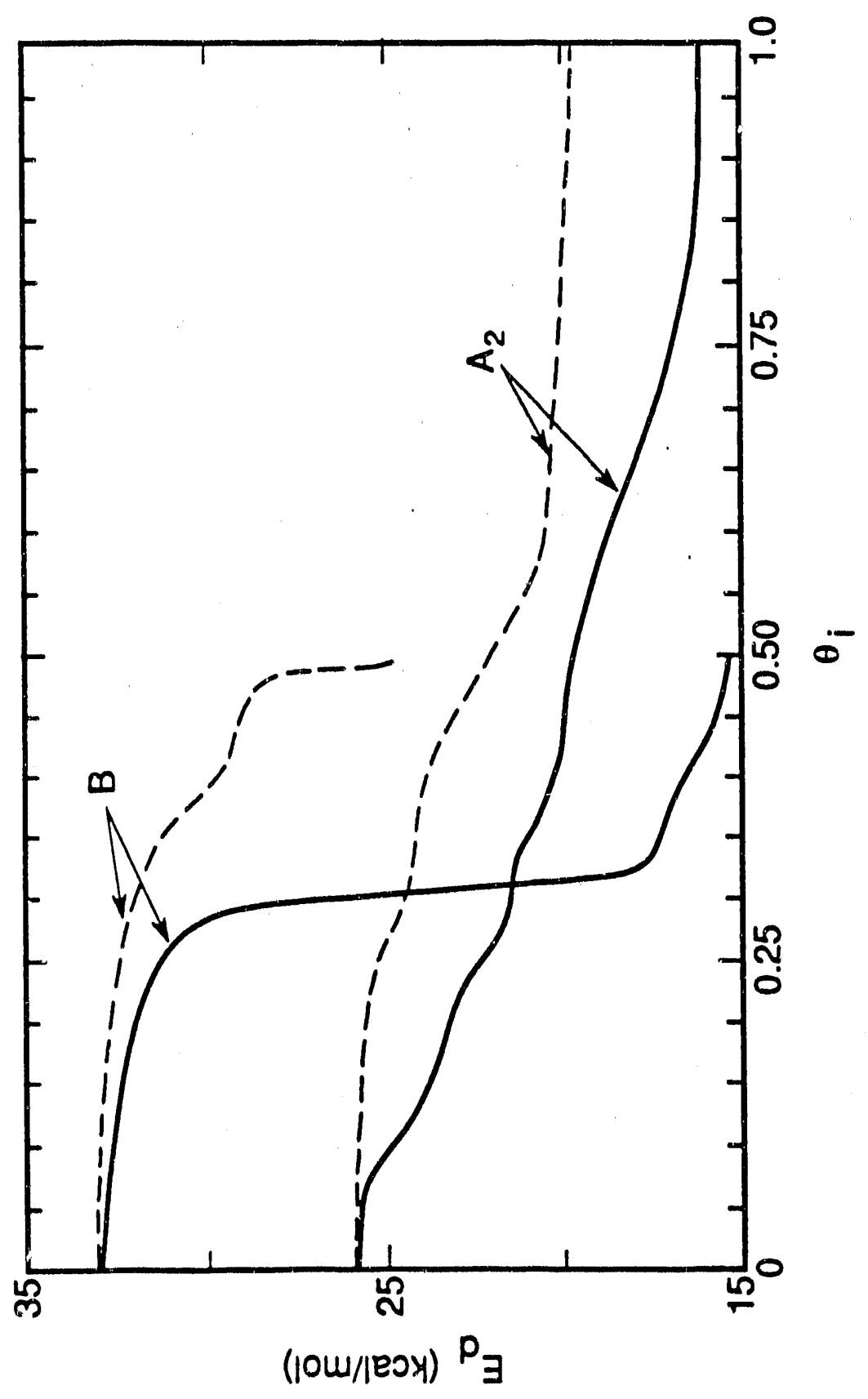

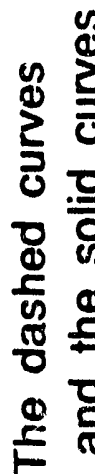

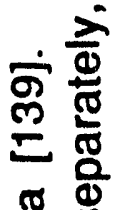

क ष

ம

은 응 웅

.5 융

ตุ

ธ్

ज के

들

‡ั

흔 등 둥

-

동

3 웡

iㅏ 응

도응 응

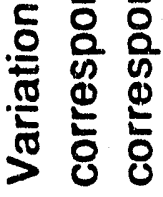

in

in 
6.0 REACTIONS

6.1 Thaoretical Prediction of $\mathrm{kr}_{\mathrm{r}}$

With the exception of absolute rate theory, none of the theoretical approaches described in Section 2 have been used to predict rate coefficients for reactions occurring on metal surfaces. Estimates from absolute rate theory of the preexponential factor $v_{r}$ for Langmuir-Hinshelwood reactions range from $10.4-104 \mathrm{~cm}^{2} \mathrm{~s}-1$ [37]. This approach suffers, however, from the need to make a number of ad hoc assumptions regarding the structure and molecular properties of the transition state. Estimates of the activation energy for surface reactions can be made using the BOC-MP approach. Shustorovich [17] has shown that where comparison can be made with experiment, the BOC-MP method provides good estimates of $E_{r}$. This conclusion is illustrated by the examples presented in Table 6.1.

6.2 Simulation of TPSR Spectra and Steady-State Dynamics

Numerous authors have reported thecretical descriptions of reactions occurring on well-defined metal surfaces and compared the results of such simulations with experiment. Two types of reaction studies have been considered. The first involves coadsorption of both reactants followed by a progressive heating of the surface to raise the temperature. The rates at which the reactants and products desorb from the surface are then followed as 
a function of time or temperature. In the second type of experiment, reactants are passed over a surface maintained at a constant temperature, and the steady-state rates of product formation are observed. Simulations of both types of experiments have been carried out using continuum models.

Bridge and Lambert [157] have used a lattice-gas model with the QCA to treat the associative recombination of $N_{s}$ and $O_{s}$ produced by dissociative adsorption of $\mathrm{NO}$ on $\mathrm{Pt}, \mathrm{Ni}$, and $\mathrm{Ru}$ surfaces. The associative desorption kinetics were simulated for two cases of interactions between the atomic adsorbates. In the first case, repulsive interactions between all atomic species $(\mathrm{N}-\mathrm{N}, \mathrm{O}-\mathrm{O}$, and $\mathrm{N}-$ O) were taken into account. In the second case, only repulsive $\mathrm{N}-\mathrm{N}$ interactions were considered. For both cases of interactions, $\mathrm{N}_{2}$ desorbed at low temperature, $\mathrm{O}_{2}$ desorbed at high temperature, and no appreciable $\mathrm{NO}$ was formed. This selectivity to $\mathrm{N}_{2}$ and $\mathrm{O}_{2}$ products was explained as follows. At low temperatures, formation of $N_{2}$ is energetically favored, and atomic nitrogen is depleted from the surface. At intermediate temperatures for which the formation of NO is energetically favorable, the amount of nitrogen remaining on the surface is too small to form appreciable NO. At still higher temperatures, atomic oxygen is the only species remaining on the surface and it desorbs as $\mathrm{O}_{2}$. The negligible selectivity to NO product formation is thus seen to result from the lack of atomic nitrogen on the surface at temperatures favorable for NO formation. Comparison of the simulated TPD spectra with those observed experimentally $[156,158,159]$ led to the conclusion that $0-0$ repulsions are significant for all three metals. In contrast, the $\mathrm{N}-\mathrm{N}$ 
interactions were found to be either zero or slightly attractive on $\mathrm{Pt}$ and $\mathrm{Ni}$, but definitely repulsive on $\mathrm{Ru}$.

The oxidation of $\mathrm{CO}$ to $\mathrm{CO}_{2}$ on an $\operatorname{lr}(110)$ surface has been examined by Zhdanov [126] with a lattice-gas/QCA model. Since the oxidation of $\mathrm{CO}$ is accompanied by $\mathrm{CO}$ desorption, both elementary processes were included in the model. The values of $v d, c o(1)=1011$ $\mathrm{s}^{-1}, E_{d}, c 0^{\circ}=33 \mathrm{kcal} / \mathrm{mol}$, and $w c o \cdot c o=2.2 \mathrm{kcal} / \mathrm{mol}$ used in these simulations were chosen to obtain a successful representation of the TPD spectrum of $\mathrm{CO}$ in the absence of adsorbed oxygen. Likewise, the values of $\mathrm{vd}, \mathrm{O} 2(2)^{2}=3.5 \times 10.4 \mathrm{~cm}^{2} \mathrm{~s}-1, E_{d, 02^{\circ}}=67$ $\mathrm{kcal} / \mathrm{mol}$, and woo $=3.6 \mathrm{kcal} / \mathrm{mol}$ were chosen to obtain a good representation of the TPD spectrum of $\mathrm{O}_{2}$ in the absence of adsorbed CO. The remaining parameters, $v_{r}=1013 \mathrm{~s}^{-1}, E_{r}{ }^{\circ}=37 \mathrm{kcal} / \mathrm{mol}$, and $w c 0.0=1.7 \mathrm{kcal} / \mathrm{mol}$, were chosen by trial and error to obtain the best representation of the TPSR spectra. A comparison between theory and experiment [160] is shown in fig. 6.1. It is seen that most features of the experimentally observed TPSR spectra are reproduced in the simulation.

As an additional part of his study, Zhdanov [126] determined the apparent activation energies for $C O$ desorption and oxidation using the following relationships:

$$
\begin{aligned}
& E_{d, \infty}=-k_{b} T \ln \left[\frac{k_{d, c o}^{(1)}}{v_{d, c o}^{(1)}}\right] . \\
& E_{r}=-k_{b} T \ln \left[\frac{k_{r}}{v_{r} 2 \theta_{c o \theta_{0}}}\right]
\end{aligned}
$$

Figure 6.2 shows that both $E_{d, c o}$ and $E_{r}$ decrease with increasing $\theta c o$ 
and $\theta_{0}$ which is a consequence of the repulsive $\mathrm{CO}-\mathrm{CO}, \mathrm{CO}-\mathrm{O}$ and $\mathrm{O}-\mathrm{O}$ interactions.

Zhdanov has also reported on the oxidation of CO over $\operatorname{Ir}(111)$ under both TPSR [161] and steady-state conditions [162]. The parameters used to simulate the TPSR spectra were $v_{r}=10^{13} \mathrm{~s}^{-1}, \mathrm{Er}_{\mathrm{r}}{ }^{\circ}$ $=31 \mathrm{kcal} / \mathrm{mol}$, and $w c 0 . c 0=0.7 \mathrm{kcal} / \mathrm{mol}$, woo $=0.8 \mathrm{kcal} / \mathrm{mol}$, and $w c 0.0=0.5 \mathrm{kcal} / \mathrm{mol}$. The experimental TPSR spectra [163] are shown in fig. 6.3a and the corresponding simulations of these spectra are presented in fig. 6.3b. Comparison of these figures indicates that the TPSR spectra determined from the lattice-gas model are in semi-quantitative agreement with those observed experimentally. Of particular interest is the fact that the latticegas model correctly predicts the strong upscale shift in the position of the $\mathrm{CO}_{2}$ peak with increasing initial oxygen coverage. This trend cannot be represented successfully if lateral interactions are neglected.

The steady-state oxidation of $\mathrm{CO}$ over $\operatorname{Ir}(111)$ was described by Zhdanov [162] in terms of the following mechanism: $\mathrm{CO}_{\mathrm{g}}=\mathrm{CO}_{\mathrm{s}}$

$\mathrm{O}_{2, \mathrm{~g}} \rightarrow 2 \mathrm{O}_{\mathrm{s}}$

$\mathrm{CO}_{\mathrm{s}}+\mathrm{O}_{\mathrm{s}} \rightarrow \mathrm{CO}_{\mathrm{g}}$

At steady state, the kinetics of the reaction are given by

$S_{C O}^{(1)}(\theta) F_{C O}=r_{d, C O}+r_{r}$

$2 S_{O_{2}}^{(2)}(\theta) F_{O_{2}}=r_{r}$

where $S_{i}(\alpha)(\theta)$ and $F_{i}$ are the sticking coefficient and the flux of component $i$, respectively. The rate parameters appearing in the 
expressions for $r_{d}, c o$ and $r$ are identical to those used for describing the oxidation of $\mathrm{CO}$ during TPSR. The dependencies of the $\mathrm{CO}$ and $\mathrm{O}_{2}$ sticking coefficients on coverage were approximated by:

$S_{c d}^{(1)}(\theta)=S_{c d}^{(1)}(0)\left(1-\theta_{c o}-\theta_{0}\right)$

$S_{O_{2}}^{(2)}(\theta)=S_{O_{2}}^{(2)}(0) P V v$

where $S_{C o}(1)(0)=1.0, S_{02}(2)(0)=0.5$, and $P V v$ is the probability that two nearest-neighbor sites are vacant. A plot of the steady-state oxidation rate as a function of temperature and pressure is shown in fig. 6.4. It is evident that the theoretical model provides a good description of the experimental data $[163,164]$, and in particular, the maximum in the rate at $600 \mathrm{~K}$.

The steady-state oxidation of $\mathrm{H}_{2}$ over $\mathrm{Pt}(111)$ has been analyzed by Zhdanov [165] using an approach similar to that used for the description of $\mathrm{CO}$ oxidation over $\operatorname{Ir}(111)$. The reaction mechanism is assumed to be:

$\mathrm{H}_{2, \mathrm{~g}}=2 \mathrm{H}_{\mathrm{s}}$

$\mathrm{O}_{2,9} \rightarrow 2 \mathrm{O}_{s}$

$\mathrm{H}_{\mathrm{s}}+\mathrm{O}_{\mathrm{s}} \rightarrow \mathrm{OH}_{\mathrm{s}}$

$\mathrm{OH}_{\mathrm{s}}+\mathrm{H}_{\mathrm{s}} \rightarrow \mathrm{H}_{2} \mathrm{O}$

Figure 6.5 compares the steady-state reaction rates observed experimentally [166] with those predicted on the basis of the latticegas model. The theoretical model provides a qualitatively correct description of the experimental data.

Sundaresan and Kaza [167] have explored the effects of limited adsorbate mobility on the rates of surface reactions. The formulation of the lattice-gas model used for this study was based 
on that used to treat the effects of adsorbate mobility on nonassociative desorption described in Section 5.3 [131]. It was demonstrated that limited adsorbate mobilities can significantly alter the rates of surface reactions depending on the lateral interactions and mobilities of the adsorbed species.

An analysis of the steady-state kinetics of the $\mathrm{CO}-\mathrm{O}_{2}$ and $\mathrm{CO}$ NO reactions over single-crystal $\mathrm{Rh}(111)$ and supported $\mathrm{Rh} / \mathrm{Al}_{2} \mathrm{O}_{3}$ has been reported by $\mathrm{Oh}$ et al. [168]. The oxidation of $\mathrm{CO}$ is represented by the mechanism described above. In the limit of high CO coverage, the authors show that the rate of $\mathrm{CO}_{2}$ formation can be written as $r_{r}=\frac{2 k_{a, O_{2}}}{k_{a, c o}} \frac{P_{O_{2}}}{P_{c o}}\left\{v_{d, c o}^{(1)} \exp \left[\frac{-\left(E_{d, c o}^{0}-\phi c o \theta c o\right)}{k_{b} T}\right]\right\}$

In this expression, the activation energy for $\mathrm{CO}$ desorption is seen to depend linearly on the coverage of $\mathrm{CO}$. With the values of $E_{d,} \mathrm{CO}^{\circ}=$ $31.6 \mathrm{kcal} / \mathrm{mol}, \phi c o=4.5 \mathrm{kcal} / \mathrm{mol}$, and $v_{d}, c o(1)=1.6 \times 1014 \mathrm{~s} \cdot 1$, a quantitative fit of rate data versus $1 / T$ could be achieved for both single crystal and supported Rh catalysts over four orders of magnitude in the rate.

In the same study, Oh et al. [168] modelled the kinetics of the CO-NO reaction for the same Rh catalysts using the following mechanism:

$$
\begin{aligned}
& \mathrm{CO}_{g}=\mathrm{CO}_{s} \\
& \mathrm{NO}_{g}=\mathrm{NO}_{s} \\
& \mathrm{NO}_{s} \rightarrow \mathrm{N}_{s}+\mathrm{O}_{s} \\
& \mathrm{NO}_{s}+\mathrm{N}_{s} \rightarrow \mathrm{N}_{2,9}+\mathrm{O}_{s} \\
& 2 \mathrm{~N}_{s} \rightarrow \mathrm{N}_{2,9} \quad \delta-\mathrm{N}_{2}
\end{aligned}
$$


$\mathrm{CO}_{s}+\mathrm{O}_{\mathrm{s}} \rightarrow \mathrm{CO}_{2, \mathrm{~g}}$

The rate of CO desorption was assumed to decrease linearly with both $\mathrm{CO}$ and $\mathrm{N}$ coverage and the rate of $\mathrm{N}_{2}$ desorption was assumed to decrease linearly with $N$ coverage. Although an analytical expression for the rate of $\mathrm{N}_{2}$ formation could not be obtained in this case, a numerical solution of the problem was achieved. Using the parameter values listed in table 6.2, good agreement was obtained between the measured and predicted rates of $\mathrm{NO}$ reduction by $\mathrm{CO}$ over a Rh(111) surface. A similar level of agreement was achieved for simulations of $\mathrm{NO}$ reduction over $\mathrm{Rh} / \mathrm{Al}_{2} \mathrm{O}_{3}$; however, in this instance a smaller value of the rate coefficient for NO dissociation was required than for $\mathrm{Rh}(111)$. The difference in the rate coefficients for $\mathrm{Rh}(111)$ and $\mathrm{Rh} / \mathrm{Al}_{2} \mathrm{O}_{3}$ was attributed to the structure sensitivity of the NO dissociation process.

\subsection{Effects of Adsorbate Islanding}

Lateral interactions between adsorbed species favor their organization into non-random, two-dimensional distributions. Under appropriate conditions, an adsorbate may even form islands of macroscopic dimensions. Such a situation can arise for coadsorbed reactants $A$ and $B$ when $W A A<0, W_{B B}=W_{A B}=0$, and $T<T_{c}$, where $T_{c}$ is the critical temperature of $A$, below which islands of $A$ form. If the reactant $B$ cannot penetrate the $A$ islands, reaction will only occur at the island boundaries. Consequently, the rate of product formation will depend on the number of $A$ islands, the length and shape of their boundaries, and on related dynamical properties such 
as the rates of island growth and diffusion of $B$. These characteristics depend in turn on initial conditions such as initial coverages, order, and time delay between $A$ and $B$ adsorption, and temperature. A well-known system exhibiting the behavior just described is $\mathrm{O}_{s}+\mathrm{CO}_{s} \rightarrow \mathrm{CO}_{2, g}$ on various metal surfaces $[2,175]$. In this case, the oxygen atoms are the aggregating species and $\mathrm{CO}$ is the mobile reactant. Both Monte Carlo and continuum models have been used to simulate the effects of island formation on reaction kinetics.

Silverberg and Ben-Shaul [176] have used a Monte Carlo model to examine the conseque :ces of the issues raised in the preceding paragraph. The reaction considered was $A_{s}+B_{s} \rightarrow A B_{g}$ with only attractive interactions between $A$ adsorbates being taken into account. In the initial segment of the simulation, species $A$ was adsorbed on the surface and then allowed to diffuse in order to either fully or partially reach an equilibrium adsorbate configuration. Species B was subsequently adsorbed randomly on unoccupied surface sites. As a consequence of the attractive interactions between $A$ adsorbates, islands of $A$ form, and the reaction occurs primarily at the ramified perimeters of the $A$ islands. The power law dependence of the reaction on the coverage of $A$ was determined to be 0.60 , which is larger than the value of 0.50 assumed for perfectly uniform islands. The rate of reaction was further shown to be sensitive to the initial coverage of reactants and to the time allowed for the $A$ species to form islands.

In a series of related studies, Silverberg and Ben-Shaul [177180] have applied a combination of Monte Carlo and lattice-gas 
models to simulate the TPSR of $A_{s}+B_{s} \rightarrow A B_{g}$. Interactions between all species $(A-A, A-B$, and $B-B)$ were included in the model. As a consequence of attractive A-A interactions, species $A$ formed islands on the surface. The Monte Carlo segment of the algorithm was used to determine the non-equilibrium, non-uniform distribution of $A$ atoms and the rate of $A B$ formation. The latticegas models were used to determine the equilibrium distribution of $B$ on surface sites not occupied by $A$. The shape and location of the simulated TPSR spectra were sensitive to the magnitude and sign of the $A-B$ and $B-B$ interactions as well as to the amount of time allowed for the formation of $A$ islands. It was observed that the two peaks in the $A B$ TPSR spectrum occurred from the reaction of $A B$ species from two types of surface environments. The lowtemperature peak corresponded to the reaction of isolated $A B$ pairs whereas the high-temperature peak corresponded to desorption of $A B$ pairs located in the vicinity of other $A$ species.

The effects of lateral interactions and phase separation on the oxidation of $\mathrm{SO}_{2}$ to $\mathrm{SO}_{3}$ over $\mathrm{Pt}$ have been investigated by Kaza and Sundaresan [181]. The reaction system was analyzed using a latticegas model with the Bragg-Williams approximation. The reaction was postulated to proceed via the following steps:

$\mathrm{O}_{2} \rightarrow 2 \mathrm{O}_{\mathrm{s}}$

$\mathrm{O}_{\mathrm{s}}+\mathrm{SO}_{2,9}=\mathrm{SO}_{3, \mathrm{~s}}$

$\mathrm{SO}_{3, \mathrm{~s}}=\mathrm{SO}_{3,9}$

It was observed that the assumption of sufficiently strong attractive interactions between adsorbed $\mathrm{SO}_{3}$ molecules resulted in a phase separation into a condensed (liquid-like) and a dilute (gas- 
like) phase. The inclusion of attractive $\mathrm{SO}_{3} \cdot \mathrm{SO}_{3}$ interactions and $\mathrm{SO}_{3}$ phase separation resulted in a qualitatively correct prediction of the dependence of the rate of $\mathrm{SO}_{2}$ oxidation on reactant partial pressures [182]. Similar agreement could not be achieved if a random distribution of $\mathrm{SO}_{3}$ was assumed.

While lattice-gas models provide a sound theoretical basis for predicting the conditions under which island formation car, occur, such models require knowledge of the strength of adsorbateadsorbate interactions. Several authors have shown that many of the consequences of island formation can be explained using Langmuir-Hinshelwood models in which the presence of islands is postulated a priori. An example of this approach is given by Barteau et al. [175], who simulated the isothermal oxidation of $\mathrm{CO}$ on a reconstructed $\mathrm{Pt}(100)-(5 \times 20)$ surface. The rate of reaction between $\mathrm{CO}$ and preadsorbed $\mathrm{O}_{2}$ was written as

$r_{r}=k_{r} \theta \operatorname{co} \theta o^{1 / 2}$

on the assumption that the reaction occurs solely at the perimeter of oxygen islands. An analytical solution to eq. 6.8 was obtained by writing species conservation equations and by assuming that the rates of $\mathrm{CO}$ adsorption and desorption were much greater than the rate of reaction. As can be seen in fig. 6.6, it was found that for a range of temperatures, the Langmuir-Hinshelwood model provides an. adequate description of the reaction dynamics.

Mukesh et al. [183] have also investigated the oxidation of $\mathrm{CO}$ at the perimeter of islands. Two models were examined. In the first first, it was assumed that $\mathrm{CO}_{s}$ aggregates into islands and is surrounded by $O_{s}$. If the number of $C O$ islands is assumed to be 
constant, independent of CO coverage, then $r_{r} \propto \theta 0(\theta c 0)^{1 / 2}$. In the second model, $\mathrm{CO}_{\mathrm{s}}$ and $\mathrm{O}_{\mathrm{s}}$ are assumed to form separate islands, and hence, $r_{r} \propto(\theta \circ \theta c 0)^{1 / 2}$. A comparison of the agreement between the $\mathrm{CO}$ islands model and experimental data obtained for supported Pt catalysts is shown in fig. 6.7. The authors note that a similar level of agreement could be achieved if both reactants were assumed to form islands.

The kinetics of $\mathrm{H}_{2}$ reacting with preadsorbed oxygen on a Pt(111) surface have been modelled by Gland et al. [184]. Oxygen was assumed to form a regular array of islands. The rate of water formation was shown to depend on the size and shape of the oxygen islands as well as on the availability of atomic hydrogen in the peripheral region around each oxygen island. The model provides a qualitatively correct description of the high reaction rates observed at low oxygen coverages, as well as the first-order dependence of the reaction rate on the hydrogen flux to the surface.

TPSR spectra of CO and NO coadsorbed on Pt(100) have been simulated by Fink et al. [185]. The reaction mechanism was identical to that given earlier for NO reduction by $\mathrm{CO}$, with the rate limiting step assumed to be the dissociation of NO. A further assumption of the model was that $\mathrm{CO}$ and $\mathrm{NO}$ adsorb into mixed islands. The simulated TPSR spectra were in good agreement with the experimentally observed spectra. In particular, the model accurately predicted the narrowness of the $\mathrm{CO}_{2}$ peak and the insensitivity of the peak position to equal coverages of coadsorbed CO and NO. 
Table 6.1

Activation energy barriers (kcal/mol) for selected surface reactions determined from the BOC-MP method [17]

\begin{tabular}{|c|c|c|c|c|}
\hline Reaction & Surface & $\begin{array}{l}\text { Activation } \\
\text { Calc. }\end{array}$ & $\begin{array}{l}\text { Energy } \\
\text { Exp. }\end{array}$ & Ref. \\
\hline $\mathrm{CO}_{2, g} \rightarrow \mathrm{CO}_{s}+\mathrm{O}_{\mathrm{s}}$ & $\begin{array}{l}\operatorname{Rh}(111) \\
\operatorname{Re}(001)\end{array}$ & $\begin{array}{l}17 \\
-5\end{array}$ & $\begin{array}{l}17 \\
50\end{array}$ & $\begin{array}{l}150 \\
151\end{array}$ \\
\hline $\mathrm{CO}_{3}+\mathrm{O}_{3} \rightarrow \mathrm{CO}_{2, \mathrm{~g}}$ & $\begin{array}{l}\operatorname{Ph}(111) \\
\operatorname{Pd}(111) \\
\operatorname{Pt}(111) \\
\mathrm{Ag}(110)\end{array}$ & $\begin{array}{l}24 \\
24 \\
23 \\
6.0\end{array}$ & $\begin{array}{l}27 \\
25 \\
25 \\
5.3\end{array}$ & $\begin{array}{r}152 \\
2 \\
2 \\
153\end{array}$ \\
\hline $\mathrm{NO}_{8}+\mathrm{N}_{3} \rightarrow \mathrm{N}_{2} \mathrm{O}_{8}$ & $\begin{array}{l}\operatorname{Rh}(111) \\
\operatorname{Rh}(100) \\
\operatorname{Pt}(111)\end{array}$ & $\begin{array}{l}22 \\
21 \\
22\end{array}$ & $\begin{array}{l}21 \\
21 \\
20\end{array}$ & $\begin{array}{l}154 \\
155 \\
156\end{array}$ \\
\hline $\mathrm{N}_{2} \mathrm{O}_{s} \rightarrow \mathrm{N}_{2, s}+\mathrm{O}_{s}$ & $\begin{array}{l}\operatorname{Rh}(111) \\
\operatorname{Pt}(111)\end{array}$ & $\begin{array}{l}.63 \\
.46\end{array}$ & - & . \\
\hline
\end{tabular}


Table 6.2

Parameters used to represent the CO-NO reaction network [168]

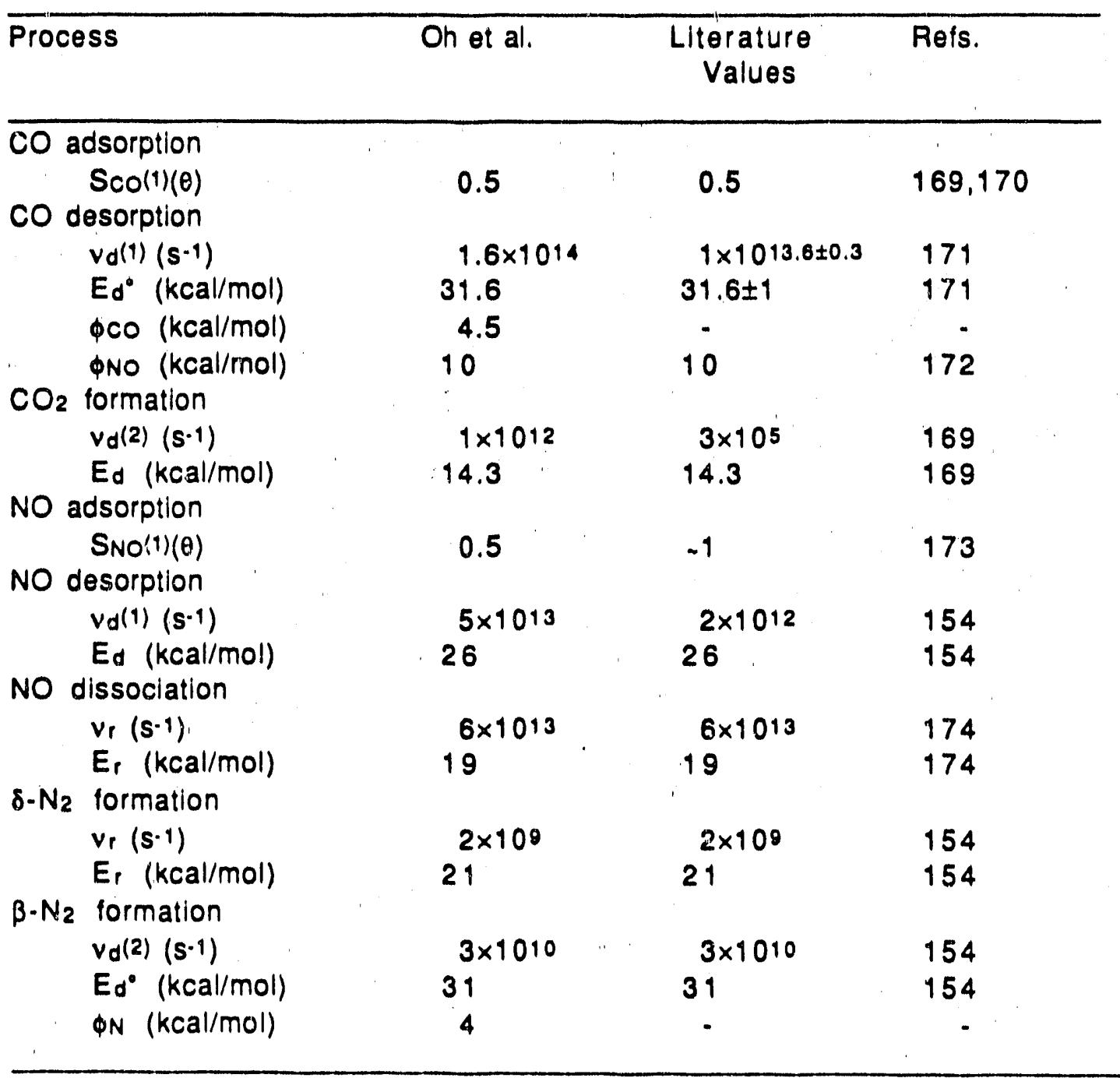




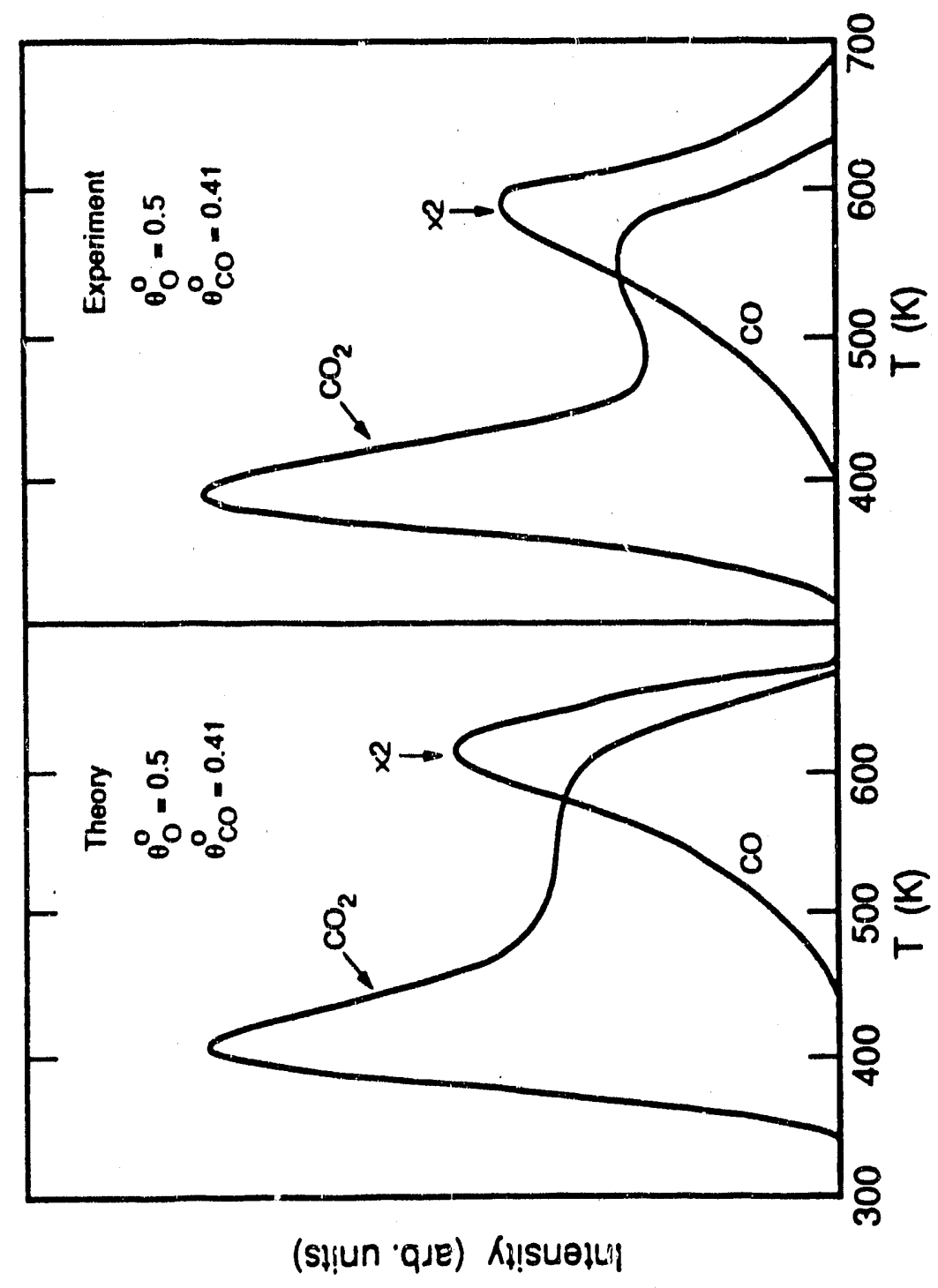

8

은

응

0

은

茓

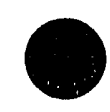

(s!fun 'QJe) Ri!suejli!

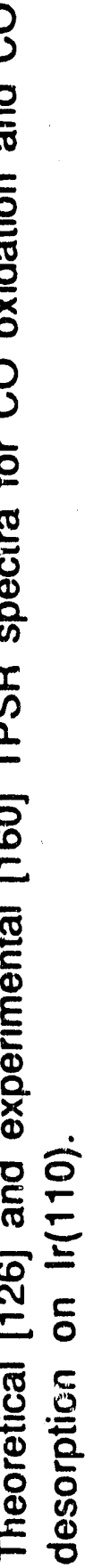

10

문 


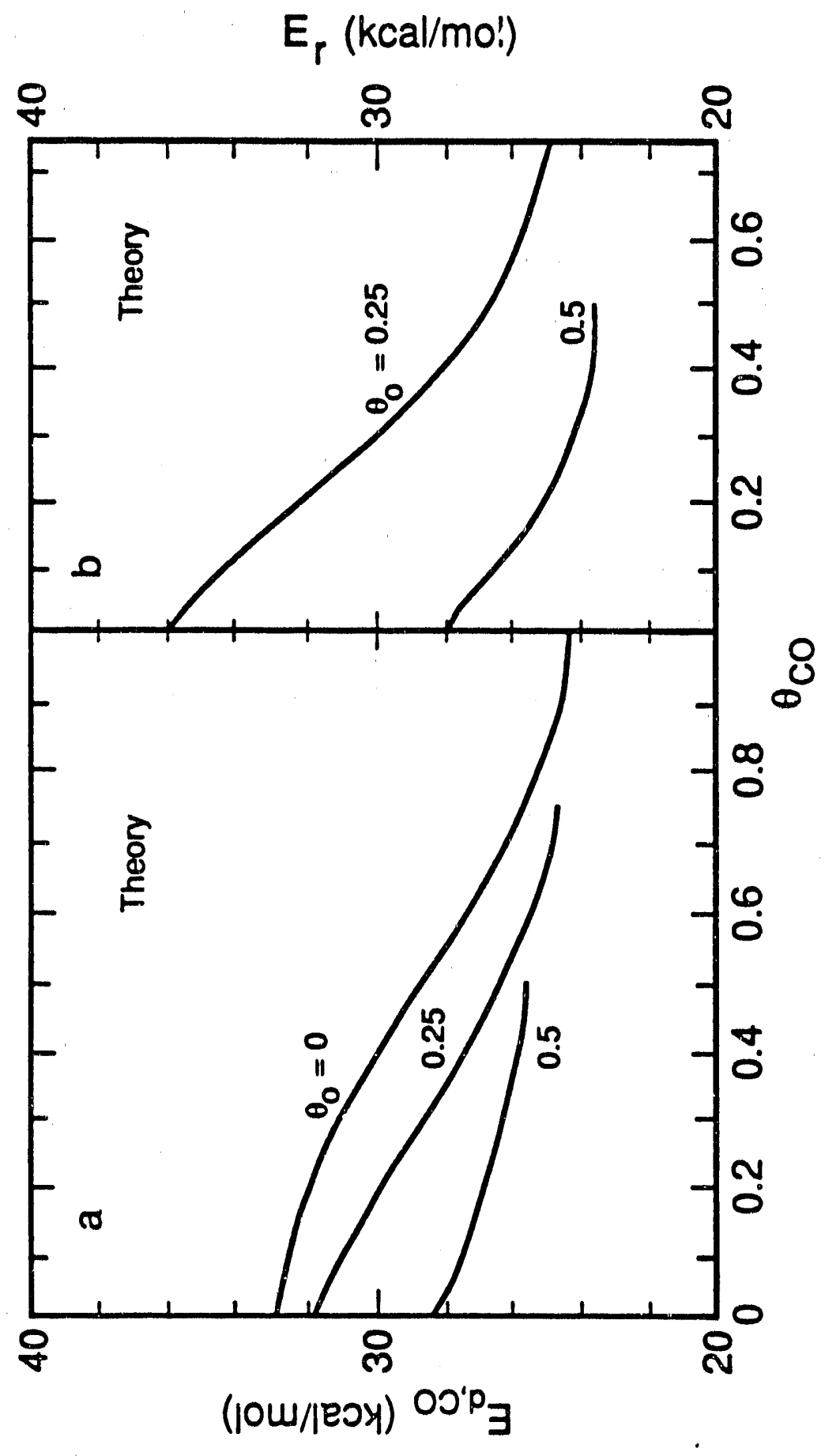

0

ஸ்

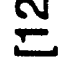

으

튼

통

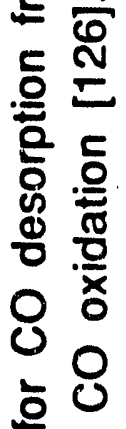

$\circ$ 농

जั

गิ

แீ

ธ

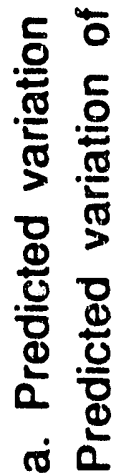

$\underset{0}{0}$

iq 

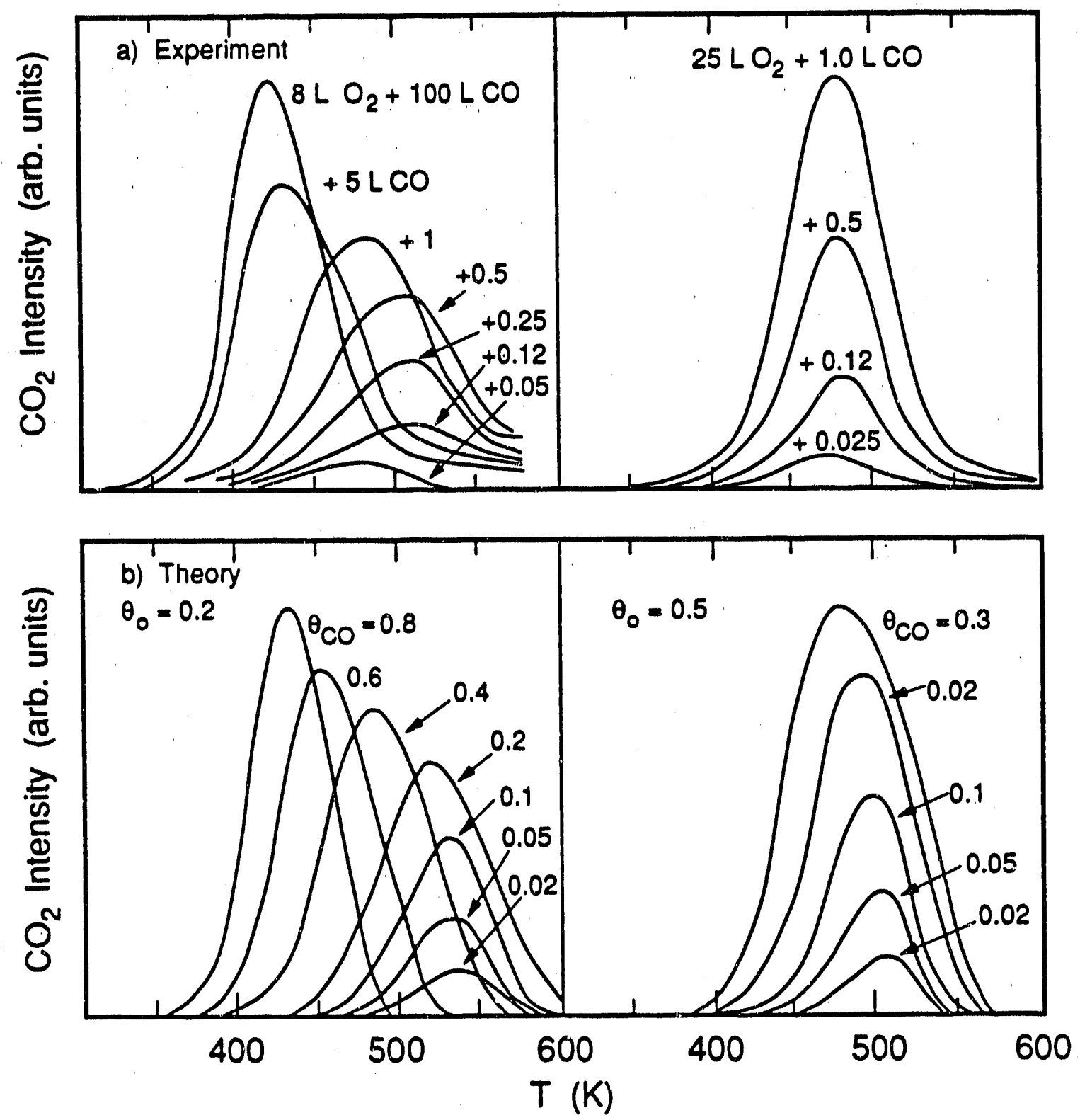

Fig. 6.3 TPSR spectra for CO oxidation on $\operatorname{Ir}(111)$. a. Experimental spectra as a function of gas exposure [163]. b. Theoretical spectra as a function of coverage [161]. 


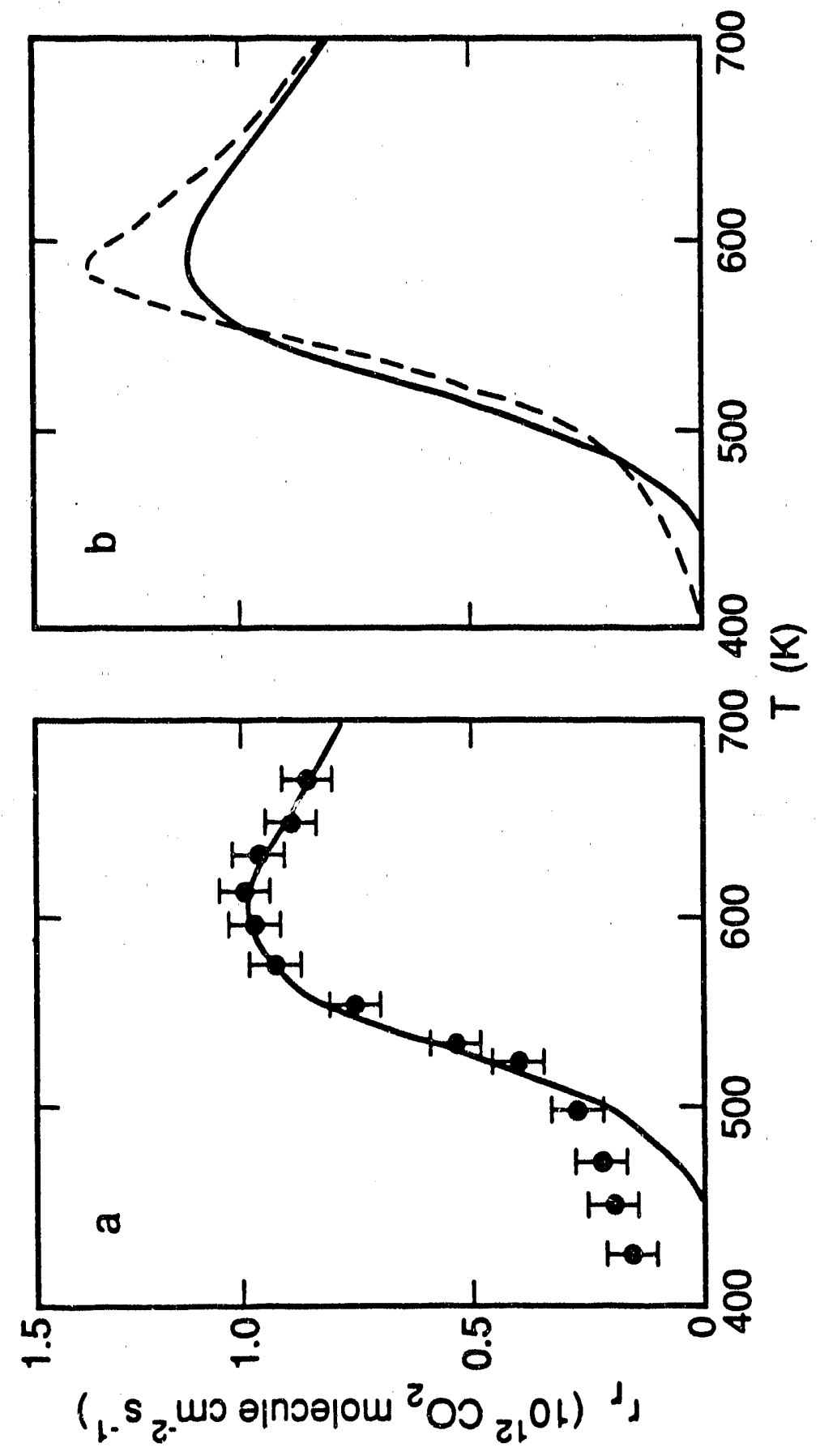

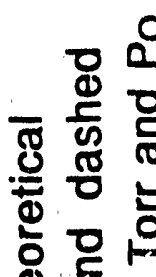

\&

뜽

ธ上

象象

응 중

- 은

다

$\underline{E}$ 드 $\frac{\dot{0}}{x}$

동 꿍

$\vdash \frac{1}{0}$

乌

क틍

ᄃ 잉.

을 爻

万ั

ํํํ कั

क 5

O

$\leftarrow$

ब

은

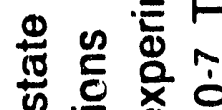

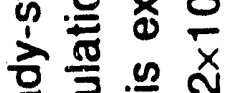

ชั

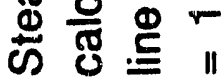

$\dot{0}$

문 


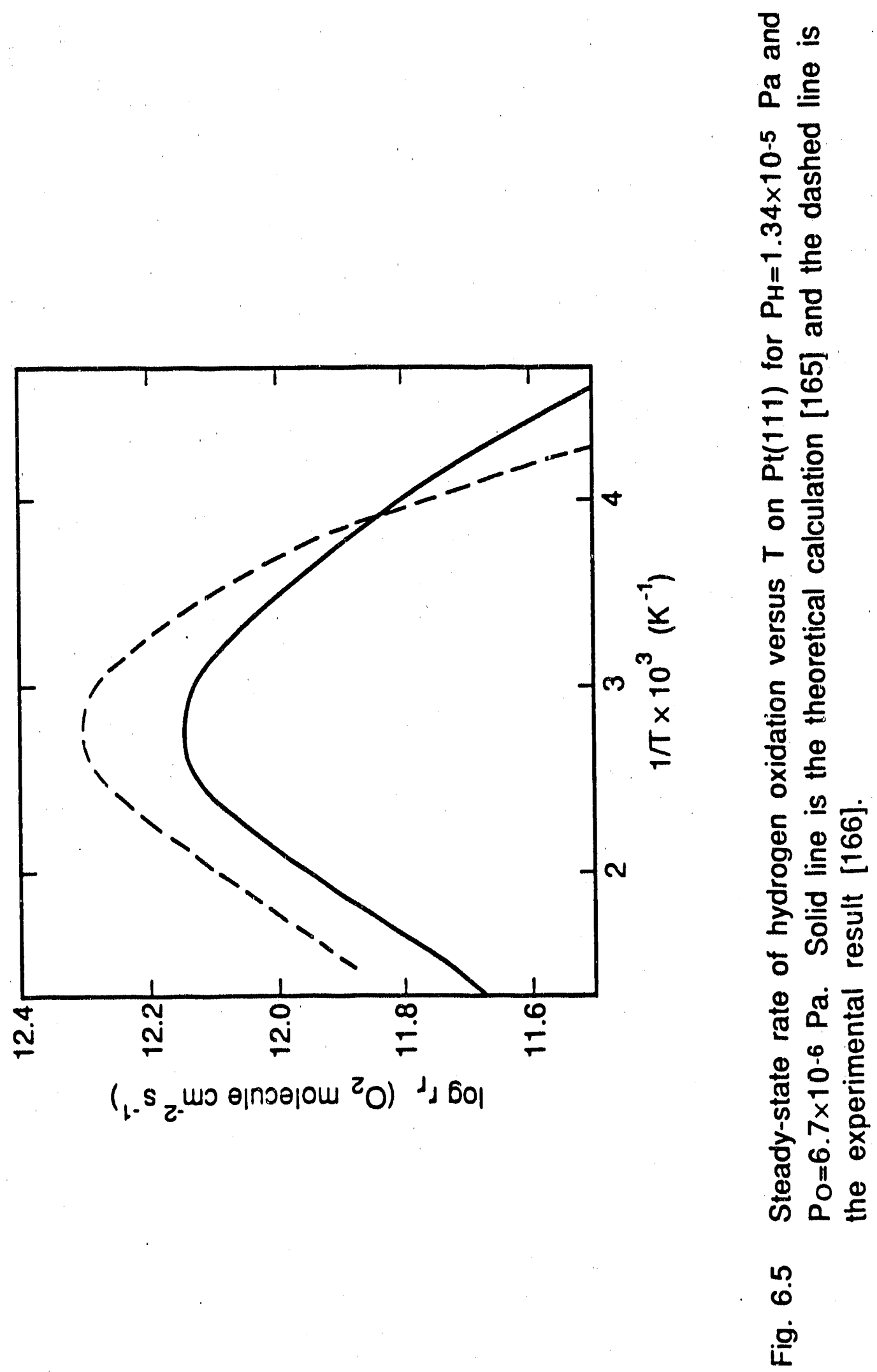




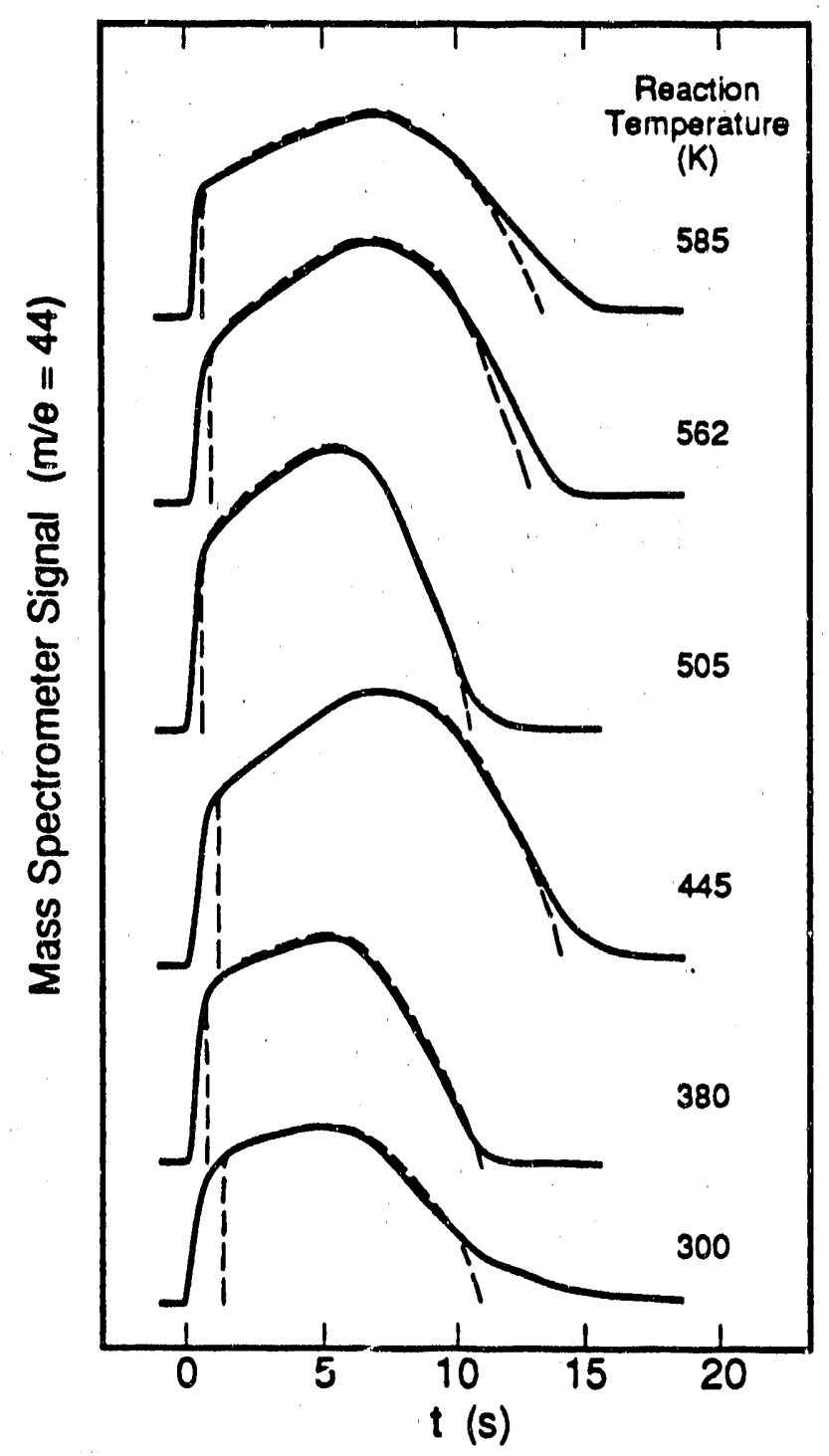

Fig. 6.6 Rate of $\mathrm{CO}_{2}$ production versus $\mathrm{t}$ for the reaction of $\mathrm{CO}$ with preadsorbed oxygen for $\mathrm{PCO}=1.0 \times 10.8$ Torr and $\theta 0 \approx 2 / 3$ monolayer [175]. Solid lines are the experimental results and the dashed lines are the model predictions. 


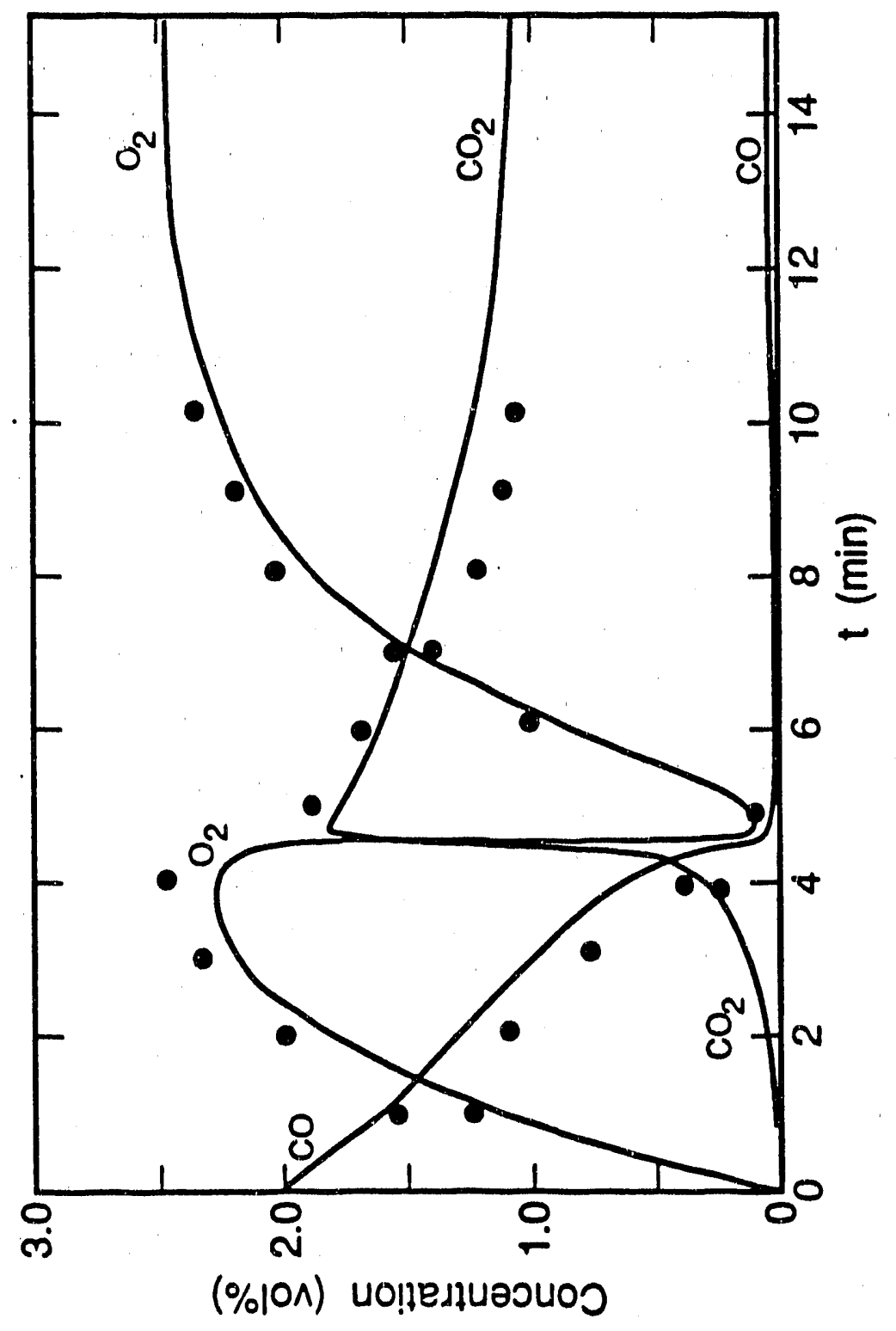

$$
\begin{aligned}
& 8 \text { 음 } \\
& \text { ○ } \\
& \$
\end{aligned}
$$$$
\text { ฉำ }
$$$$
\text { 돈 은 }
$$$$
\text { 章 }
$$$$
\text { 잉 }
$$$$
\text { 읐 릉 }
$$$$
\text { क }
$$$$
\text { 힘 }
$$$$
\text { c }
$$$$
\text { 도 올 }
$$$$
\text { ㄴำ }
$$$$
\text { 동 일 }
$$$$
\text { ல }
$$$$
\text { 을 }
$$$$
\text { - 논 }
$$$$
\text { 롤 둥 }
$$$$
\text { ज ह 은 }
$$$$
\text { 广 }
$$$$
\text { ( } 0 \text { \% }
$$$$
\text { ญ ํํำ }
$$$$
\text { 항 }
$$$$
\text { 은 은 }
$$$$
\text { 등 을 }
$$$$
\text { 질 }
$$$$
\text { 동 요 }
$$$$
\text { 它 }
$$$$
\text { N }
$$$$
\text { iํㅛ }
$$ 


\subsection{CONCLUDING REMARKS}

Considerable progress has been made in developing theoretical methods for predicting the dynamics of elementary processes occurring on metal surfaces. The starting point in all cases is a description of the potential governing the interactions between gas molecules and the atoms at the surface of the metal. At high adsorbate coverages, additional information must be supplied to describe the effects of adsorbate-adsorbate interactions. Accurate potential functions based on a quantum treatment of gas-metal interactions are not yet available and consequently, resort must be made to empirical potential functions. While such functions can be constructed in an ad hoc fashion, there do not appear to be generally accepted criteria for establishing the suitability of such functions for dynamic calculations. At a minimum, it would seem that the potential function chosen should give the experimentally observed heat of adsorption and the vibrational frequency for adsorbate-metal vibrations in the ground state.

As noted in Section 2, both classical and quantum descriptions of the dynamics of gas-metal interactions have been developed. At this time, only the classical approaches offer a way of predicting rate and diffusion coefficients for a wide range of processes and adsorbed species. Direct simulation of molecular dynamics is practical provided that the process of interest is $\sim 103$ times slower than the time constant for the fastest mode of motion (e.g., vibration). Experience has shown that molecular dynamics simulation provides estimates of the diffusion coefficient and of 
the sticking coefficient for dissociative adsorption of diatomic molecules that are in good quantitative agreement with experiment. By use of either MD or stochastic (Lanjevin) dynamics, it is also possible to include the effects of lattice vibrations. Where this has been done, it has been found that lattice vibrations do affect the calculated values of the sticking and diffusion coefficients.

The constraints of molecular dynamics with respect to describing the dynamics of infrequent events for which the time constants are $>10.9 \mathrm{~s}$ can be overcome through the use of dynamically corrected TST. This approach provides a rigorously correct theoretical framework for calculating rate and diffusion coefficients. Dynamically corrected TST provides a rational basis for identifying conditions under which precursor states affect the adsorption and desorption of adsorbates. Calculations of diffusion coefficients and rate coefficients for desorption obtained by means of dynamically corrected TST show good quantitative agreement with experimental measurements. Moreover, this theoretical approach explains why desorption rate coefficients exhibit a deviation from Arrhenius behavior at high temperatures, and why the rate coefficients for the desorption of molecular species are a factor of 100-1000 larger than those for the desorption of atomic species.

Absolute rate theory is useful only for crude estimation of preexponeritial factors, in as much as ad hoc assumptions regarding the transition state structure are necessary in order to estimate partition functions. This represents a severe limitation which limits the accuracy of the estimates of the preexponential factor to 
within a factor of 10 to 103 .

Several theoretical models based on quantum mechanics have been developed. Such models can include the exchange of energy between the adsorbate and the surface as well as the effects of barrier tunneling and reflection. For small molecules and atoms (e.g., $H_{2}$ and $H$ ), quantum effects such as barrier tunneling and reflection are observed. For more massive adsorbates, quantum effects are projected to be less important.

The effects of lateral interactions can be neglected at low adsorbate coverages but can become significant at higher coverages. Such interactions can alter both the spatial distribution of adsorbates on a metal surface and the apparent activation energy barriers. To date, only empirical or semiempirical representations of lateral interactions have been developed which are capable of describing rate or diffusion coefficients for a wide range of surface processes and adsorbate coverages. The simplest and the most frequently used approach is to treat lateral interactions by a sum of pairwise additive contributions to the activation energy. With this approach, both the magnitude and sign of the interaction are treated as adjustable parameters. An alternative technique for describing lateral interactions is the BOC-MP method. In this case, interactions between adsorbates arise as a consequence of throughmetal and direct adsorbate-adsorbate interactions.

Analytical expressions for the kinetics of adsorption, diffusion, desorption, and reaction in the presence of lateral interactions can be written using a lattice-gas model with the quasichemical approximation. While this approach captures the effects of 
lateral interactions, the lattice-gas model requires two rather restrictive assumptions. The first is that lateral interactions can be represented by a sum of pairwise contributions of equivalent strength and the second is that adsorbates always maintain an equilibrium configuration on the metal surface.

An alternative approach to account for the effects of lateral interactions is to incorporate the energetics predicted either from the sum of pairwise additive contributions or from the BOC-MP method into Monte Carlo simulations. The Monte Carlo models treat the kinetics of surface processes in terms of probabilities which are specific to each site and its local environment, and thus require no assumptions about the distribution of adsorbates on the surface.

The present review has shown that both continuum and Monte Carlo models can be used to account for the experimentally observed kinetics of surface processes. In addition, the temperatureprogrammed desorption and reaction of adsorbates on metal surfaces can also be described with these techniques. Proper representation of lateral interactions has led to the conclusion that the appearance of multiple peaks in TPD and TPSR spectra are a consequence of such interactions.

Both continuum and Monte Carlo models have been used to describe the effects of island formation on the dynamics of surface reactions. Continuum models require assumptions to be made about the number, size, and shape of the islands. Such models have been successful, though, in representing the effects of adsorbate islands on reaction dynamics. By contrast, Monte Carlo models make no assumptions about the concentration of islands or their size and 
shape. To date, though, kinetics predicted with Monte Carlo models have not been compared extensively with experimental observation.

Theoretical methods for predicting rate coefficients for other than very simple surface reactions have yet to be developed. The principal difficulty is the absence of accurate methods for generating the relevant potential energy hypersurface. Should it become possible to generate such functions, then the desired rate coefficients could be calculated using dynamically corrected TST. For reactions involving the removal or addition of a hydrogen atom, quantum effects might be expected to be important.

It is evident from this review that theoretical methods are now available for explaining many of the phenomena observed when gases interact with metal surfaces. One area for future research is the development of realistic potential hypersurfaces for describing surface reactions. Such calculations should preferably be based on $a b$ initio quantum chemical methods, to the extent possible. Another area that should be considered is the influence of adsorbate coverage on the reconstruction of metal surfaces and the effects of surface reconstruction on the dynamics of elementary processes occurring on metal surfaces. Finally, the suitability of stochastic models for describing reaction kinetics over a wide range of conditions should be explored more fully. 
NOMENCLATURE
$A, A_{2}, B, A B$ Adsorbates
$A^{*}, A_{2}{ }^{*} \quad$ Precursor species
$A_{s}$
Chemisorbed species
A-A Adsorbate-adsorbate interaction
a
Morse potential parameter
as Area per reaction site
C
Concentration
D Diffusivity
$D(\theta) \quad$ Coverage-dependent diffusivity
$D_{A B} \quad$ Dissociation energy of $A B$
Do Preexponential factor for diffusion
d Dimensionality
E Activation energy
Ea Activation energy for adsorption
Ed Activation energy for desorption
Ediff Activation energy for diffusion
Ediss Activation energy for dissociation
$\mathrm{Er}_{\mathrm{r}} \quad$ Activation energy for reaction
Es Lateral interaction energy
$E_{a^{\circ}} \quad$ Activation energy for adsorption in the absence of lateral interactions
$E_{d^{\circ}} \quad$ Activation energy for desorption in the absence of lateral interactions
$E_{r^{\circ}} \quad$ Activation energy for reaction in the absence of lateral interactions
Et Energy of the transition state
Eo Energy of the reactant
ET Iricident kinetic translational energy
$F \quad$ Flux of adsorbate
$f_{p} \quad$ Correction factor for reaction due to lateral interactions
is Correction term in TST
$f_{a}{ }^{\prime}, f_{a}{ }^{n} \quad$ Correction factors for adsorption due to lateral interactions
$f^{\prime}, f^{\prime \prime} \quad$ Correction factors for desorption due to lateral interactions
G Normalization constant
$\mathrm{H} \quad$ Classical Hamiltonian 


\begin{tabular}{|c|c|}
\hline H & Quantum Hamiltonian operator \\
\hline h & Planck's constant \\
\hline J & Diffusive flux \\
\hline K & $\mathrm{ka}^{*} / \mathrm{kd}^{*}$ \\
\hline k & rate coefficient \\
\hline kb & Boltzmann constant \\
\hline ka & Rate coefficient for adsorption \\
\hline$k d(\alpha)$ & Rate coefficient for desorption \\
\hline kTST & Rate coefficient from TST \\
\hline $\mathrm{ka}^{*}$ & Rate coefficient for adsorption from the precursor state \\
\hline $\begin{array}{l}K d^{*} \\
L\end{array}$ & $\begin{array}{l}\text { Rate coefficient for desorption from the precursor state } \\
r_{d} * /\left(r_{a}{ }^{*}+r d^{* *}\right)\end{array}$ \\
\hline LEPS & London-Eyring-Polanyi-Sato potential energy surface \\
\hline 1 & Effective length of an adsorbate \\
\hline$M-A$ & Metal-adsorbate interaction \\
\hline$m$ & Molecular weight or mass of an adsorbate \\
\hline $\mathrm{mB}$ & Molecular weight of species B \\
\hline mi & Number of adsorbates bonded to ith metal atom \\
\hline $\mathrm{m}^{\prime}$ & Mass of a surface atom \\
\hline $\mathrm{N}$ & Normalization constant \\
\hline No & Number of species which desorb \\
\hline Ns & Number of surface metal atoms \\
\hline$n$ & Number of nearest neighbors \\
\hline$n^{\prime}$ & Number of metal atoms bonded to an adsorbate \\
\hline$P$ & Probability \\
\hline$P(p, q)$ & Probability density in phase space \\
\hline$P_{B}$ & Pressure of species B \\
\hline$P_{n}(t)$ & Probability of finding an adsorbate in state $n$ at time \\
\hline PAA & $\begin{array}{l}\text { Probability that two nearest-neighbor sites are occupied } \\
\text { by } A\end{array}$ \\
\hline PAV & $\begin{array}{l}\text { Probability that of two nearest-neighbor sites are } \\
\text { occupied, one is occupied by } A \text { and the other is vacant }\end{array}$ \\
\hline PW & Probability that two nearest-neighbor sites are vacant \\
\hline$P_{A, n}$ & $\begin{array}{l}\text { Probability that a site occupied by } A \text { has } n \text { nearest } \\
\text { neighbors }\end{array}$ \\
\hline$P_{A B ; 1}$ & $\begin{array}{l}\text { Probability that a pair of sites occupied by } A \text { and } B \text { has } \\
\text { the environment } i\end{array}$ \\
\hline$P_{i}$ & Probability of desorption from the ith site \\
\hline$P_{i j}$ & Probability of diffusion from site $i$ to site $j$ \\
\hline
\end{tabular}




\begin{tabular}{|c|c|}
\hline $\begin{array}{l}p \\
Q_{, Q} \\
Q^{\circ} \\
Q_{0 A} \\
Q_{A, n} \\
Q_{A, n}(1) \\
Q_{A, n^{(2)}} \\
Q_{A, n^{*}}\end{array}$ & $\begin{array}{l}\text { Position } \\
\text { Heat of adsorption } \\
\text { Heat of adsorption in the absence of lateral interactions } \\
\text { Heat of adsorption of } A \text { in the on-top position } \\
\text { Heat of adsorption of } A \text { as a function of coordination } \\
\text { Heat of adsorption of } A \text { due to } M-A \text { interactions } \\
\text { Heat of adsorption of } A \text { due to A-A interactions } \\
\text { Total heat of adsorption of } A\end{array}$ \\
\hline $\mathbf{q}$ & Momentum \\
\hline qo & Partition function for adsorbates in the transition state \\
\hline & $\begin{array}{l}\text { Partition function for adsorbates in the transition state } \\
\text { Random number }\end{array}$ \\
\hline $\mathbf{R}$ & Random force \\
\hline$r, r i$ & Distance \\
\hline ra & Rate of adsorption \\
\hline rd & Rate of desorption \\
\hline ro & Equilibrium bond distance \\
\hline$r r$ & Rate of reaction \\
\hline $\mathbf{S}$ & Dividing plane \\
\hline$S(\alpha)(\theta)$ & Sticking coefficient \\
\hline$S(\alpha)(0)$ & Sticking coefficient at zero coverage \\
\hline $\begin{array}{l}\text { So }(\alpha) \\
\text { So }\end{array}$ & $\begin{array}{l}\text { Preexponential factor for adsorption } \\
\text { Reactive sticking coefficient }\end{array}$ \\
\hline s & Reaction coordinate \\
\hline So & Location of the dividing plane \\
\hline$T$ & Temperature \\
\hline$T_{c}$ & Critical temperature \\
\hline $\begin{array}{l}\text { Ts } \\
\text { TST }\end{array}$ & $\begin{array}{l}\text { Temperature of the surface } \\
\text { Transition state theory }\end{array}$ \\
\hline$t$ & time \\
\hline Uo & $\begin{array}{l}\text { Velocity required for an adsorbate located at so to } \\
\text { desorb }\end{array}$ \\
\hline V & Potential \\
\hline VOA & Equilibrium energy \\
\hline$V_{p}$ & Interaction potential in the primary zone \\
\hline$V_{s}$ & Interacticn potential in the secondary zone \\
\hline$v$ & Mean velocity \\
\hline vs & Velocity in the $s$ direction \\
\hline W & Potential of mean force \\
\hline
\end{tabular}




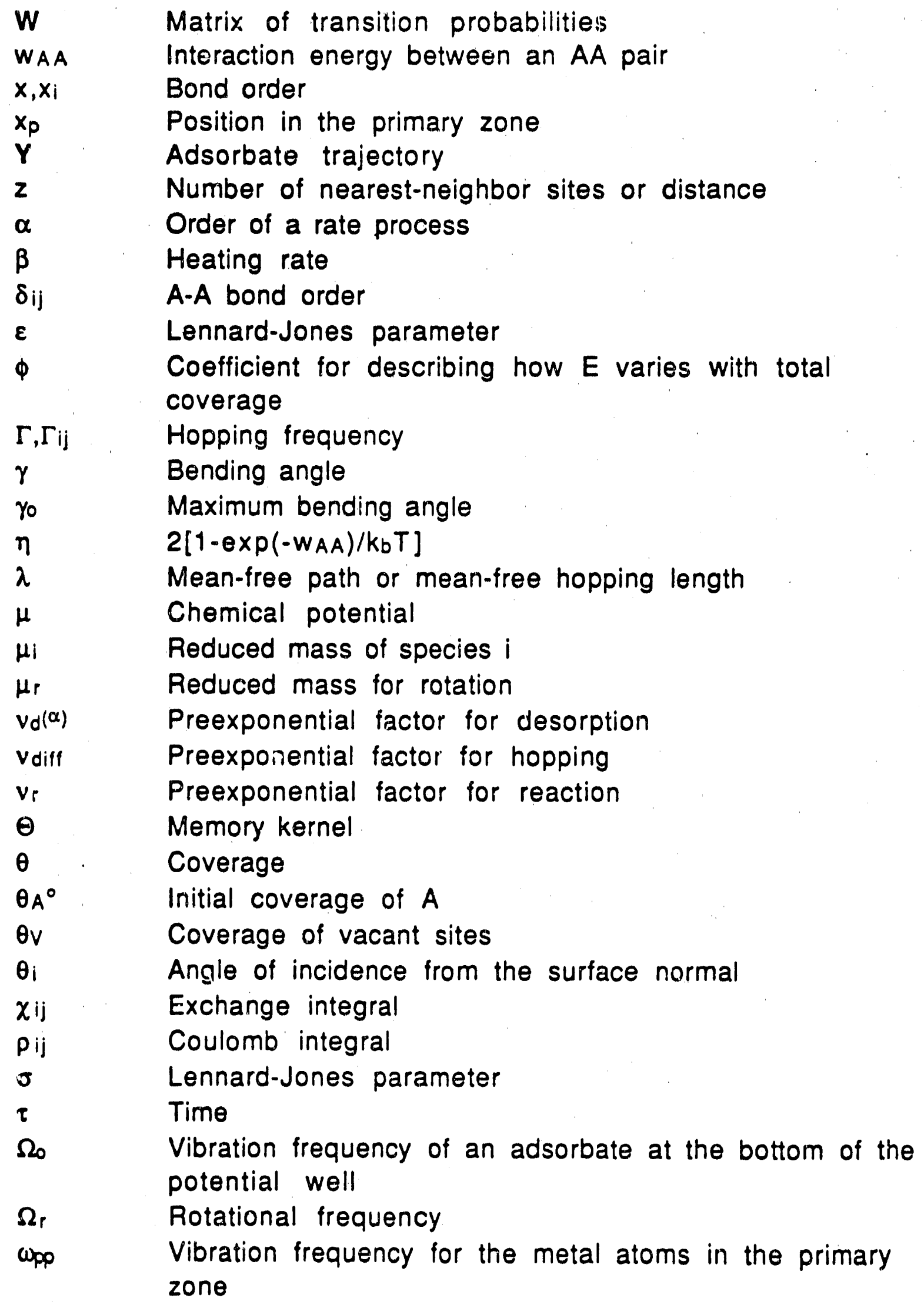


$\xi \quad$ Correction factor in TST

$\psi$ Wave function

$\zeta \quad$ Trapping probability 
REFERENCES

[1] M.A. Morris, M. Bowker, and D.A. King, in: Comprehensive Chemical Kinetics, Simple Processes at the Gas-Solid Interface, Vol. 19, Eds. C.H. Bamford, C.F.H. Tipper and R.G. Compton (Elsevier, Amsterdam,1984) ch. 1.

[2] G. Ertl in: Catalysis, Science and Technology, Vol. 4, Eds. J.R. Anderson and M. Boudart (Springer-Verlag, Berlin, 1983) ch. 3.

[3] G.A. Somorjai, Chemistry in Two Dimensions: Surfaces (Cornell University Press, Ithaca, NY 1981).

[4] G. Ehrlich and K. Stolt, Ann. Rev. Phys. Chem. 31 (1980) 603.

[5] A. Kapoor, R.T. Yang and C. Wong, Catalysis Rev.-Sci. Eng. 31 (1989) 129.

[6] L.D. Schmidt, Catalysis Rev.-Sci. Eng. 9 (1974) 115.

[7] D.A. King, Surf. Sci. 47 (1975) 384.

[8] R.J. Madix, in: The Chemical Physics of Solid Surfaces and Heterogeneous Catalysis, Vol. 4, Eds. D.A. King and D.P. Woodruff (Elsevier, Amsterdam, 1982) ch. 1.

[9] J.C. Tully, Ann. Rev. Phys. Chem. 31 (1980) 319.

[1C] S.A. Adelman and J.D. Doll, Acc. Chem. Res. 10 (1977) 378.

[11] J.D. Doll and A.F. Voter, Ann. Rev. Phys. Chem. 38 (1987) 413.

[12] For example, see Refs. [30-33,42].

[13] T.B. Grimley, in: The Chemical Physics of Solid Surfaces and Heterogeneous Catalysis, Vol. 2, Eds. D.A. King and D.P. Woodruff (Elsevier, Amsterdam, 1983) ch. 5.

[14] T.B. Grimley, in: The Nature of the Surface Chemical Bond, Eds. T.N. Rhodin and G. Ertl (North-Holland, Amsterdam, 1979) ch.1

[15] E. Shustorovich, Surf. Sci. Rep. 6 (1986) 1.

[16] E. Shustorovich, Acc. Chem. Res. 21 (1988) 189.

[17] E. Shustorovich, in: Advances in Catalysis, Vol 37, Eds. D.D. Eley, H. Pines and P.B. Weisz (Academic Press, New York) in press.

[18] W.H. Weinberg in: Springer Series in Surface Sciences 8, Eds.

M. Grunze and H.J. Kreuzer (Springer-Verlag, Berlin, 1987) 94.

[19] R. Gorte and L.D. Schmidt, Surf. Sci. 76 (1978) 559.

[20] A. Cassuto and D.A. King, Surf. Sci. 102 (1981) 388.

[21] P.J. Kisliuk, J. Phys. Chem. Solids 3 (1957) 95; 5 (1958) 78.

[22] D.A. King, Surf. Sci. 64 (1977) 43.

[23] K. Schönhammer, Surf. Sci. 83 (1979) L633.

[24] V.P. Zhdanov, Surf. Sci. 102 (1981) L35. 
[25] V.P. Zhdanov, Surf. Sci. 111 (1981) 63.

[26] V.P. Zhdanov, Surf. Sci. 111 (1981) L662.

[27] S.A. Adelman and J.D. Doll, J. Chem. Phys. 61 (1974) 4242; 62 (1975) 2518; 64 (1976) 2375.

[28] E.K. Grimmelmann, J.C. Tully and E. Helfand, J. Chem. Phys. 74 (1981) 5300.

[29] J.C. Tully, Surf. Sci. 111 (1981) 461.

[30] A. Gelb and M.J. Cardillo, Surf. Sci. 59 (1976) 128; Surf. Sci. 64. (1977) 197.

[31] A. Gelb and M.J. Cardillo, Surf. Sci. 75 (1978) 199.

[32] C. Chiang and B. Jackson, J. Chem. Phys. 87 (1987) 5497.

[33] C. Lee and A.E. DePristo, J. Chem. Phys. 85 (4986) 4161.

[34] C. Lee and A.E. DePristo, J. Chem. Phys. 87 (1987) 1401.

[35] C. Lee and A.E. DePristo, J. Vac. Sci. Technol. A 5 (1987) 485.

[36] D.J. Doren and J.C. Tully, Langmuir 4 (1988) 256.

[37] V.P. Zhdanov, J. Pavlicek and Z. Knor, Catal. Rev.-Sci. Eng. 30 (1988) 501.

[38] O.V. Krylov, M.U. Kisliuk, B.R. Shub, A.A. Gezalov, N.D. Maksimova and Y.N. Rufov, Kinetika i kataliz, 13 (1972) 598.

[39] R.C Baetzold and G.A Somorjai, J. Catalysis 45 (1976) 94.

[40] K.J. Laidler, S. Glasstone and H. Eyring, J. Chem. Phys. 8 (1940) 659; 8 (1940) 667.

[41] Y. Zeira, A. Redondo and W.A. Goddard III, Surf. Sci. 131 (1983) 221; 136 (1984) 41; J. Vac. Sci. Technol. B 2 (1984) 550.

[42] B. Jackson and H. Metiu, J. Chem. Phys. 86 (1987) 1026.

[43] S. Efrima, C Jedrzejek, K.F. Freed, E. Hood and H. Metiu, J. Chem. Phys. 79 (1983) 2436.

[44] E. Hood, C Jedrzejek, K.F. Freed and H. Metiu, J. Chem. Phys. 81 (1984) 3277.

[45] K.F. Freed, J. Chem. Phys. 82 (1985) 5264.

[46] C.R. Arumainayagam, R.J. Madix, M.C. McMaster, V.M. Suzawa and J.C. Tully, Surf. Sci. 226 (1990) 180.

[47] C.W. Muhlhausen, L.R. Williains and J.C. Tully, J. Chem. Phys. 83 (1985) 2594.

[48] I. NoorBatcha, L.M. Raff, and D.L.Thompson, J. Chem. Phys. 81 (1984) 3715.

[49] M. Balooch, M.J. Cardillo, D.R. Miller and R.E. Stickney, Surf. Sci. 46 (1974) 358.

[50] M.J. Cardillo, M. Balooch and R.E. Stickney, Surf. Sci. 50 (1975) 
263.

[51] A.V. Hamza and R.J. Madix, J. Phys. Chem. 89 (1985) 5381.

[52] H.J. Robota, W. Vielhaber, M.C. Lin, J. Segner and G. Ertl, Surf. Sci. 155 (1985) 101.

[53] A. Kara and A.E. DePristo, Surf. Sci. 193 (1988) 437.

[54] H.E. Pfnür, C.T. Rettner, J. Lee, R.J. Madix and D.T. Auerbach, J. Chem. Phys. 85 (1986) 7452.

[55] T. Truong, G. Hancock and D.G. Truhlar, Surf. Sci. 214 (1989) 523.

[56] J.E. Adams and J.D. Doll, Surf. Sci. 103 (1981) 472; 111 (1981)

492.

[57] M. Asscher, O.M. Becker, G. Haase and R. Kosloff, Surf. Sci. 206 (1988) L880.

[58] C.T. Rettner and H. Stein, Phys. Rev. Letters 59 (1987) 2768.

[59] D. A. King and M.G. Wells, Proc. R. Soc. Lond. A 339 (1974) 245.

[60] V.P. Zhdanov, Surf. Sci. 209 (1989) 523.

[61] E.S. Hood, B.H. Toby and W.H. Weinberg, Phys. Rev. Letters 55 (1985) 2437.

[62] P. Feulner and D. Menzel, Phys. Rev. B 25 (1982) 4295.

[63] D. Menzel, H. Pfnür and P. Feulner, Surf. Sci. 126 (1983) 374.

[64] H.K. McDowell and J.D. Doll, J. Chem. Phys. 78 (1983) 3219.

[65] J.D. Doll and H.K. McDowell, J. Chem. Phys. 77 (1982) 479.

[66] J.D. Doll and H.K. McDowell, Surf. Sci. 123 (1982) 99.

[67] H.K. McDowell and J.D. Doll, Surf. Sci. 121 (1982) L537.

[68] D.W. Bassett and M.J. Parsley, Brit. J. Appl. Phys. 2 (1969) 13.

[69] G. Ayrault and G. Ehrlich, J. Chem. Phys. 60 (1974) 281.

[70] P. Cowan and T.T. Tsong, Phys. Letters 53A (1975) 383.

[71] W.R. Graham and G. Ehrlich, Thin Solid Films 25 (1975) 85;

Phys. Rev. Letters 31 (1973) 1407.

[72] P.P. Cowan and T.T. Tsong, 22nd Intern. Field Emission Symp., Atlanta, 1975.

[73] P.G. Flahive and W.R. Graham, Surf. Sci. 91 (1980) 463.

[74] J.D. Doll and D.L. Freeman, Surf. Sci. 134 (1983) 769.

[75] R.L. Palmer, J. Vac. Sci. Technol. 12 (1975) 1403.

[76] M.T. Martin and J. B. Hudson, J. Vac. Sci. Technol. 15 (1978) 474.

[77] S.M. Levine and S.H. Garofalini, Surf. Sci. 167 (1986) 198.

[78] H.C. Abbink, R.M. Broudy and G.P. McCarthy, J. Appl. Phys, 39 
(1968) 4673.

[79] A.F. Voter and J.D. Doll, J. Chem. Phys. 82 (1985) 80.

[80] S.M. Valone, A.F. Voter and J.D. Doll, Surf. Sci. 155 (1985) 687.

[81] J. G. Lauderdale and D.G. Truhlar, Surf. Sci. 164 (1985) 558.

[82] S.M. Valone, A.F. Voter and J.D. Doll, J. Chem. Phys. 85 (1986) 7480 .

[83] J. G. Lauderdale and D.G. Truhlar, J. Chem. Phys. 84 (1986) 1843.

[84] T.N. Truong and D.G. Truhlar, J. Phys. Chem. 91 (1987) 6229.

[85] T.N. Truong and D.G. Truhlar, J. Chem. Phys. 88 (1988) 6611.

[86] C.H. Mak and S.M. George, Chem. Phys. Lett. 135 (1987) 381.

[87] T.N. Truong, D.G. Truhlar, J.R. Chelikowsky and M.Y. Chou, J. Phys. Chem. 94 (1990) 1973.

[88] C.H. Mak, J.L. Brand, A.A. Deckert and S.M. George, J. Chem. Phys. 85 (1986) 1676.

[89] A. Auerbach, K.F. Freed and R. Gomer, J. Chem. Phys. 86 (1987). 2356.

[90] R. DiFoggio and R. Gomer, Phys. Rev. B 25 (1982) 3490.

[91] S.C. Wang and R. Gomer, J. Chem. Phys. 83 (1985) 4193.

[92] K.B. Whaley, A. Nitzan and R.B. Gerber, J. Chem. Phys. 84 (1986) 5181.

[93] D.A. Reed and G. Ehrlich, Surf. Sci. 102 (1981) 588.

[94] V.P. Zhdanov, Surf. Sci. 149 (1985) L13.

[95] V.P. Zhdanov, Surf. Sci. 194 (1988) 1.

[96] M. Metropolis, A.W. Rosenbluth, A.N. Teller and E. Teller, J. Chem. Phys. 21 (1953) 1087.

[97] K. Kawasaki, Phys. Rev. B 145 (1966) 224; 148 (1966) 375; 150 (1966) 285.

[98] H.C. Kang and W.H. Weinberg, J. Chem. Phys. 90 (1989) 2824.

[99] M. Bowker and D.A. King, Surf. Sci. 71 (1978) 583.

[100] M. Bowker and D.A. King, Surf. Sci. 72 (1978) 208.

[101] R. Butz and G. Wagner, Surf. Sci. 63 (1977) 448.

[102] M. Tringides and R. Gomer, Surf. Sci. 145 (1984) 121.

[103] J.R. Chen and R. Gomer, Surf. Sci. 79 (1979) 413.

[104] A. Sadiq and K. Binder, Surf. Sci. 128 (1983) 350.

[105] C.H. Mak, H.C. Andersen and S.M.George, J. Chem. Phys. 88 (1988) 4052.

[106] T. Engel and R. Gomer, J. Chem. Phys. 52 (1970) 5572.

[107] B.E. Nieuwenhuys, D.Th. Meijer and W.M.H. Sachtler, Phys. 
Status Solidi (a) 24 (1974) 115.

[108] R.J. Gorte, L.D. Schmidt and J.L. Gland, Surf. Sci. 109 (1981) 367.

[109] L.M. Raff, I. NoorBatcha and D.L. Thompson, J. Chem. Phys. 85 (1986) 3081.

[110] K.D. Brzoska and Ch. Kleint, Thin Solid Films 34 (1976) 131.

[111] Yu. I. Belyakov, N.I. Ionov and T.N. Kompaniets, Sov. Phys. Solid State A 1 (1973) 2567.

[112] G. Schulze and M. Henzler, Surf. Sci. 124 (1983) 336.

[113] L. Schmidt and R. Gomer, J. Chem. Phys. 42 (1965) 3573.

[114] L.M. Kahn and S.C. Ying, Solid State Commun. 16 (1975) 799.

[115] G. Ehrlich, in: Advances in Catalysis, Vol. 14, Eds. D.D. Eley, H. Pines and P.B. Weisz (Academic Press, New York, 1963) 255.

[116] C.R. Helms and R.J. Madix, Surf. Sci. 52 (1975) 677.

[117] J.E. Lenriard-Jones and C. Strachan, Proc. Roy. Soc. Lond. A 150 (1935) 442.

[118] C. Strachan, Proc. Roy. Soc. Lond. A 150 (1935) 456.

[119] J.E. Lennard-Jones and A.F. Devonshire, Proc. Roy. Soc. Lond. A 156 (1936) 6; 156 (1936) 29; 158 (1937) 242; 158 (1937) 253.

[120] A.F. Devonshire, Proc. Roy. Soc. Lond. A 156 (1936) 37; 158 (1937) 269.

[121] J.E. Lennard-Jones and A.F. Devonshire, Nature 137 (1936) 1069.

[122] F.O. Goodman, Surf. Sci. 24 (1971) 667.

[123] G.S. De, U. Landman and M. Rasolt, Phys. Rev. B 21 (1980) 3256.

[124] C.G. Goymour and D.A. King, J. Chem. Soc. Faraday Trans. 16 (1973) 749.

[125] C.G. Goymour and D.A. King, J. Chem. Soc. Faraday Trans. 16 (1973) 736.

[126] V.P. Zhdanov, Surf. Sci. 123 (1982) 106.

[127] J.L. Taylor and W.H. Weinberg, Surf. Sci. 78 (1978) 259.

[128] J.L. Taylor, D.E. Ibbotson and W.H. Weinberg, Surf. Sci. 79 (1979) 349.

[129] A.H. Smith, R.A. Barker and P.J. Estrup, Surf. Sci. 136 (1984) 327.

[130] A.V. Myshlyavtsev and V.P. Zhdanov, J. Chem. Phys. 92 (1990) 3909.

[131] S. Sundaresan and K.R. Kaza, Surf. Sci. 160 (1985) 103. 
[132] S.J. Lombardo and A.T. Bell, Surf. Sci. 206 (1988) 101.

[133] J.L. Sales and G. Zgrablich, Phys. Rev. B 35 (1987) 9520.

[134] A. Córdoba and J.J. Luque, Phys. Rev. B 26 (1982) 4028.

[135] J.L. Sales, G. Zgrablich and V.P. Zhdanov, Surf. Sci. 209 (1989) 208.

[136] H.R. Han and L.D. Schmidt, J. Phys. Chem. 75 (1971) 227.

[137] P.J. Estrup, Department of Chemistry, Brown University, unpublished results.

[138] D. Gupta and C.S. Hirtzel, Chem. Phys. Letters 149 (1988) 527; Surf. Sci. 210 (1989) 322.

[139] S.J. Lombardo and A.T. Bell, Surf. Sci. 224 (1989) 451.

[140] E.I. Ko, Department of Chemical Engineering, Carneige-Mellon University, unpublished results.

[141] J. Benziger and R.J. Madix, Surf. Sci. 94 (1980) 119.

[142] J.M. White, J. Phys. Chem. 87 (1983) 915.

[143] Y. Kim, H.C. Peebles and J.M. White, Surf. Sci. 114 (1982) 363.

[144] A.K. Galwey, in: Advannces in Catalysis, Vol 26, Eds. D.D. Eley,

H. Pines and P.B. Weisz (Academic Press, New York, 1977) 247.

[145] H.C. Kang, T.A. Jachimowski and W.H. Weinberg, to appear in J. of Chem. Phys.

[146] E.G. Seebauer, A.C.F. Kong and L.D. Schmidt, Surf. Sci. 193 (1988) 417.

[147] M. Alnot and A. Cassuto, Surf. Sci. 112 (1981) 325.

[148] P.J. Estrup, E.F. Greene, M.J. Cardillo and J.C. Tully, J. Phys. Chem. 90 (1986) 4099.

[149] J.B. Miller, H.R. Siddiqui, S.M. Gates, J.N. Russell, Jr., J.T. Yates, Jr., J.C. Tully and M.J. Cardillo, J. Chem. Phys. 87 (1987) 6725.

[150] D.W. Goodman, D.E. Peebles and J.M. White, Surf. Sci. 140 (1984) L239.

[151] H. Peled and M. Asscher, Surf. Sci. 183 (1987) 201.

[152] W.H. Weinberg, Surf. Sci. 128 (1983) L224.

[153] M. Bowker, M.A. Barteau and R.J. Madix, Surf. Sci. 92 (1980) 528.

[154] T.W. Root, L.D. Schmidt and G.B. Fisher, Surf. Sci. 134 (1983) 30.

[155] P. Ho and J.M. White, Surf. Sci. 137 (1984) 103.

[156] C.M. Comrie, W.H. Weinberg and R.M. Lambert, Surf. Sci. 57 
(1976) 619.

[157] M.E. Bridge and R.M. Lambert, Proc. R. Soc. Lond. A 370 (1980) 545.

[158] H. Conrad, G. Ertl, J. Küppers and E.E. Latta, Surf. Sci. 50 (1975) 296.

[159] P.D. Reed, C.M. Comrie and R.M. Lambert, Surf. Sci. 72 (1978) 423.

[160] J.L. Taylor, D.E. Ibbotson and W.H. Weinberg, Surf. Sci. 90 (1979) 37.

[161] V.P. Zhdanov, Surf. Sci. 137 (1984) 515.

[162] V.P. Zhdanov, Surf. Sci. 169 (1986) L278.

[163] J. Küppers and A. Plagge, Z. Naturforsch. 34a (1979) 81.

[164] V.P. Ivanov, G.K. Boreskov, V.I. Savchenko, V.L. Tataurov, W.H.

Weinberg and W.F. Egelhoff, Dokl. Akad. Nauk. SSSR, 249 (1979) 622, in Russian.

[165] V.P. Zhdanov, Surf. Sci. 169 (1986) 1.

[166] V.A. Sobyanin, G.K. Boreskov and A.R. Cholach, Dokl. Akad. Nauk. SSSR, 278 (1984) 1422, in Russian.

[167] S. Sundaresan and K.R. Kaza, Chem. Eng. Commun. 35 (1985) 1.

[168] S.H. Oh, G.B. Fisher, J.E. Carpenter, and D.W. Goodman, J. of Catalysis 100 (1986) 360.

[169] C.T. Campbell, S.-K. Shi and J.M. White, Appl. Surf. Sci. 2 (1979) 382.

[170] C.T. Campbell and J.M. White, J. Catalysis 54 (1978) 289.

[171] F.A. Thiel, E.D. Williams, J.T. Yates, Jr. and W.H. Weinberg, Surf. Sci. 84 (1979) 54.

[172] T.W. Root, G.B. Fisher and L.D. Schmidt, Surf. Sci. 150 (1985) 173.

[173] C.T. Campbell and J.M. White, Appl. Surf. Sci. 1 (1978) 347.

[174] T.W. Root, G.B. Fisher and L.D. Schmidt, J. Chem. Phys. 85 (1986) 4679.

[175] M.A. Barteau, E.I. Ko and R.J. Madix, Surf. Sci. 104 (1981) 161.

[176] M. Silverberg, A. Ben-Shaul and F. Rebentrost, J. Chem. Phys. 83 (1985) 6501.

[177] M. Silverberg and A. Ben-Shaul, J. Chem. Phys. 87 (1987) 3178.

[178] M. Silverberg and A. Ben-Shaul, Chem. Phys. Leiters 134 (1987) 491.

[179] M. Silverberg and A. Ben-Shaul, Surf. Sci. 214 (1989) 17.

[180] M. Silverberg and A. Ben-Shaul, J. Stat. Phys. 52 (1988) 1179. 
[181] K.R. Kazá and S. Sundaresan, Chem. Eng. Commun. 32 (1985) 333.

[182] C.G. Vayenas and H.M. Saltsburg, J. Catalysis 57 (1979) 206.

[183] D. Mukesh, W. Morton, C.N. Kenney and M.B. Cutlip, Surf. Sci. 138 (1984) 237.

[184] J.L. Gland, G.B. Fisher and E.B. Kollin, J. of Catalysis 77 (1982) 263.

[185] Th. Fink, J.-P. Dath, M.R. Bassett, R. Imbihl and G. Ertl, to appear in Vacuum. 


\title{
Chapter III
}

\section{A Monte Carlo Model for the Simulation of Temperature- Programmed Desorption Spectra}

\begin{abstract}
A Monte Carlo model has been developed for describing the temperature-programmed desorption of adsorbates from single crystal surfaces. This model takes into account the effects of surface diffusion and the influence of metal-adsorbate (M-A) and adsorbate-adsorbate (A-A) interactions on the coverage dependence of the activation energy for desorption. The inclusion of $M-A$ and $A$ A interactions has a pronounced effect on the shape of the predicted TPD spectrum. Where only a single peak is observed in the absence of $\mathrm{M}-\mathrm{A}$ and $\mathrm{A}-\mathrm{A}$ interactions, multiple peaks are found when these interactions are included. The inclusion of $M-A$ and $A-A$ interactions is also shown to produce a nonlinear decline in the activation energy for desorption as a function of increasing adsorbate coverage.

Simulated TPD spectra for CO desorption from a Pd(100) surface and for $\mathrm{H}_{2}$ desorption from $\mathrm{Mo}(100)$ and $\mathrm{Ni}(111)$ surfaces, obtained using the Monte Carlo model, are in satisfactory agreement with those observed experimentally. An important feature of the model is that it describes correctly the observed dependence of the activation energy on adsorbate coverage.
\end{abstract}




\subsection{INTRODUCTION}

Temperature-programmed desorption (TPD) spectroscopy is widely used to characterize the interactions of adsorbates with metal surfaces [1-4]. The analysis and interpretation of TPD spectra have usually been carried out using rate expressions which assume the surface and adlayer to be homogeneous for a given adsorbate coverage. In such models, the rate of desorption is given by the product of a rate coefficient and an integer power dependence on the surface coverage, $\theta$. The rate coefficient is written as the product of a preexponential factor, $\mathrm{kd}^{\circ}$, and an exponential function, the argument of which contains the activation energy for desorption, Ed. While the earliest modeling efforts assumed $k_{d}{ }^{\circ}$ and $E_{d}$ to be independent of $\theta$, more recent studies have demonstrated that both parameters can be coverage dependent.

Analyses of experimental TPD spectra indicate that $E_{d}$ generally decreases with increasing adsorbate coverage. Most attempts to account for this trend have been based on a lattice-gas model with pairwise-additive energetics [5-10]. In this model, adsorbed species are considered to be localized on a twodimensional surface and interactions between nearest-neighbor adsorbates are assigned either an attractive or repulsive contribution to the total binding energy of the adsorbate. Simulations of TPD spectra based on pairwise-additive energetics with either the quasichemical approximation or with Monte Carlo methods have been successful in accounting qualitatively (and in a few instances, quantitatively) for deviations from the assumption of non-interacting adsorbates [6-10]. It should be noted, though, that 
the use of pairwise-additive energetics is not physically satisfying, since no a priori basis exists for assigning the relative magnitude and sign of an interaction or for assuming linearly additive contributions from nearest-neighbor adsorbates. Furthermore, the assumption of a homgeneous surface in the quasichemical approximation is not warranted. For a given global coverage, different local configurations of adsorbates may exist on the surface and hence the value of $E_{d}$ for each environment would not be equivalent.

Different dependencies of $k d^{\circ}$ on $\theta$ have been reported by various authors, some indicating that $k d^{\circ}$ increases with $\theta$, and others, that $k_{d}{ }^{\circ}$ decreases with $\theta$ [11-17]. Attempts to explain such trends have been made using absolute rate theory. While this approach does permit for a rationalization of why $k d^{\circ}$ should be coverage dependent, it has not proven to be adequate for making accurate predictions of $k_{d}{ }^{\circ}$.

The objective of this study is to examine the influence of adsorbate-metal interactions, adsorbate-adsorbate interactions, and surface mobility on the rates of adsorbate desorption from welldefined surfaces. A lattice-gas model approach is used. Rather than rely on average or mean-field coverage effects, however, the influence of local environment is taken into account explicitly. The local binding energy for an adsorbate is determined using the BondOrder-Conservation (BOC) method [18]. This method allows one to account for the coordination of the adsorbate with the surface and the effects of nearest-neighbor adsorbates. In the latter case, both through-metal and adsorbate-adsorbate interactions are included. 
BOC calculations of the local heat of adscrption and activation energy for desorption are then incorporated into a Monte Carlo simulation of temperature-programmed desorption. The method is used to examine the desorption of $\mathrm{CO}$ from a $\mathrm{Pd}(100)$ surface and $\mathrm{H}_{2}$ desorption from $\mathrm{Mo}(100)$ and $\mathrm{Ni}(111)$ surfaces. An interesting feature of the model is that it describes not only the shape of the TPD spectrum, but also the dependence of the desorption activation energy and the distribution of local surface site occupancy on the average surface coverage.

\subsection{THEORY}

Wo sssume that at any moment in time adsorbate atoms or molecules occupy well-defined sites on a single-crystal metal surface, the coordination of the adsorbate being specific to the metal and the adsorbate. The number of metal atoms coordinated to a single adsorbate is 1 for an on-top site, 2 for a bridge site, 3 for a three-fold hollow, and 4 for a four-fold hollow. The coverage is defined as the number of adsorbate atoms or molecules, $N$, divided by the number of atoms at the surface of the metal, $N_{s}$ :

$\theta=N / N_{s}$

Adsorbed atoms or molecules are assumed to participate in only two rate processes: surface diffusion and desorption. Since the local coverage varies with position on the surface, it is anticipated that the dynamics of surface diffusion and desorption should be site specific. This specificity will be accounted for through the local heat of adsorption and activation energy for desorption, as discussed below. 
The probability of desorption from the local environment $i$ in the time interval $\Delta t$ can be defined as $P_{i}^{(1)}=k_{d, i}^{0} \exp \left[-E_{d, i} / R T\right] \Delta t$

where $k_{d, j^{\circ}}$ is the frequency factor for desorption and $E_{d, i}$ is the activation energy for desorption. The value of $\Delta t$ in eq. 2 must be sufficiently small so that $P_{i}(1)$ goes to unity at a temperature sufficiently high to guarantee virtually complete desorption from all sites of type $i$. This point is discussed more fully in the next section.

The rate of desorption, expressed as a turnover frequency based on $\mathrm{N}_{s}$, is $r_{d}=N_{d} /\left(\alpha N_{s} \Delta t\right)$

where $\mathrm{Nd}_{d}$ is the number of adsorbate atoms or molecules desorbing in the time interval $\Delta t$. The parameter $\alpha$ is 1 for molecular desorption and 2 for associative desorption of atoms. Consequently, $\mathrm{rd}$ is the rate of desorption observed from the gas phase. The total rate of desorption from all local environments is simply $r_{d}=\sum_{i} N_{d, i} /\left(\alpha N_{s} \Delta t\right)$

$$
=N_{d} /\left(\alpha N_{s} \Delta t\right)
$$

Since the activation energy ior surface diffusion is $10-15 \%$ of that for desorption, whereas the frequency factors for the two processes are comparable, surface diffusion is expected to be a much more rapid process than desorption. As a consequence, we assume that adsorbate atoms or molecules will reposition themselves nearly instantaneously to achieve an equilibrium state. The probability that an adsorbate in site $\mathrm{i}$ moves to an adjacent 
vacant site $\mathrm{j}$ is given by [19]:

$P_{i j}^{(2)}=\frac{\exp \left[-\left(Q_{i}-Q_{i}\right) / R T\right]}{1+\exp \left[-\left(Q_{i}-Q_{i}\right) / R T\right]}$

where $Q_{i}$ and $Q_{j}$ are the heats of adsorption for an adsorbate at sites $i$ and $j$, respectively. Equation 5 weights diffusion jumps according to the magnitude of $\left(Q_{i}-Q_{j}\right)$. It should be noted that if $Q_{i}=Q_{j}, P_{i j}(2)=$ 0.5 , which indicates that two sites of equal energy have equal probability of occupancy. For a sufficiently large number of jumps, application of eq. 5 yields an equilibrium distribution of adsorbates on the metal surface.

Having developed expressions for the probabilities of adsorbate desorption and surface diffusion, we proceed next to indicate hov: $E_{d, i}$ and $\alpha_{i}$ appearing in these expressions, are calculated. Our approach is to use the Bond-Order-Conservation (BOC) method developed by Shustorovich, since it allows us to account for the effects of local site occupancy without the introduction of locally assigned energy parameters [18].

A principal assumption of the BOC method is that the twocenter interaction between an adsorbate atom $A$ and a surface metal atom $M$ can be described by a Morse potential

$Q(x)=Q_{0}\left(2 x-x^{2}\right)$

where $x$ is the bond order and $Q_{0}$ is the equilibrium value of the M-A bond energy for $x=1$. The bond order in eq. 6 is defined as

$x=\exp \left[-\left(r-r_{0}\right) / a\right]$

where $r$ is the M-A bond length, $r_{0}$ is the equilibrium bond length, and $a$ is a scaling parameter [18]. When $A$ interacts with more than one metal atom, the total heat of adsorption is given by the sum of all 
two-center interactions. A second assumption central to the BOC method is that along a reaction path describing the interactions of a molecular or atomic species with a metal surface, the total bond order is conserved and normalized to unity.

Using the above assumptions, Shustorovich [18] has shown that the heat of chemisorption of an atom $A$ interacting with $n$ metal atoms is given by

$Q_{A, n}=Q_{O A}(2-1 / n)$

The heat of adsorption for a molecular adsorbate $A B$ coordinated via atom $A$ to $n$ metal atoms can be approximated by $Q_{A B, n}=\frac{Q_{O A}^{2}}{D_{A B}+Q_{O A} / n}$

where $D_{A B}$ is the gas-phase dissociation energy for the $A-B$ bond. Equations 8 and 9 are appropriate for isolated adsorbate atoms or molecules. When the adsorbate coverage increases, however, situations arise in which more than one adsorbate is coordinated with a single metal atom. In this case, the heat of adsorption is given by $\dot{Q_{A, n}}=\sum_{i=1}^{n} \frac{Q_{A, n}}{n m_{i}}\left(2-1 / m_{i}\right)$

where $\mathrm{mi}$ is the number of adsorbates bonded to the ith metal atom.

At high coverages, direct A-A interactions can become significant and must be taken into account in calculating $Q_{A}, n^{\circ}$. To do so, the total bond order associated with $A$ is still normalized to unity but partitioned between the $A-A$ and $M-A$ interactions. The resulting expression for $Q_{A, n^{*}}$ can be written as 
$\dot{Q_{A, n}}=Q_{A, n}^{(1)}+Q_{A, n}^{(2)}$

where $Q_{A, n}(1)$ is the heat of adsorption due to $M-A$ interactions and $Q_{A, n}(2)$ is the heat of adsorption due to $A-A$ interactions.

The value of $Q_{A, n}(2)$ can be expressed as

$Q_{A, n}^{2)}=\sum_{i=1}^{n} \sum_{l=1}^{L} 0.5 D_{A A}\left(2 . \delta_{i l}-\delta_{i l}^{2}\right)$

where $D_{A A}$ is the bond dissociation energy for the A-A bond and $\delta_{i l}$ is the bond order for the A-A interaction between the $A$ atom coordinated with metal atom $i$ and the ith nearest-neighbor atom which is also coordinated with metal atom $\mathrm{i}$. The summation over 1 in eq. 12 is to account for all nearest-neighbor $A$ atoms. The occurrence of A-A interactions weakens the bond order associated with $M-A$ interactions and as a consequence, the bond order for each component of an $\mathrm{Mn}_{\mathrm{n}} \mathrm{A}$ bond is given by

$x_{i, n}=\frac{1}{n} \cdot \sum_{i=1}^{L} \delta_{i l}$

Equation 13 is then used to calculate $Q_{A, n}(1)$, which is given by $Q_{A, n}^{(1)}=\sum_{i=1}^{n}\left[\frac{Q_{O A}}{m_{i}}\left(2-\frac{1}{m_{i}}\right)\left(2 x_{i, n}-x_{i, n}^{2}\right)\right]$

The activation energy for desorption depends on the mode of desorption. For the desorption of a single atom or an adsorbed molecule, the activation energy for desorption is identical to the heat of adsorption. For homonuclear associative desorption, the activation energy, $E_{d, A A}$ is given by [18]

$E_{u, A^{\prime}}=\left(\dot{Q}_{A, n} \dot{Q}_{A^{\prime}, n}\right) !\left(\dot{Q}_{A^{\prime}, n}+\dot{Q}_{A^{\prime}, n}^{*}\right)$ 
where $Q_{A, n^{*}}$ and $Q_{A^{\prime}, n^{*}}$ are calculated using eq. 10 or 11 . The two recombining atoms are designated as $A$ and $A^{\prime}$ to denote that the local environments of the two atoms may be different.

The magnitude of the preexpmontial factor, $k d, i^{\circ}$, can be estimated froin absolute rate theory $[1-17]$. The accuracy of these estimates is, in general not high, since numerous assumptions mus! be made to evaluate the partition functions for the adsorbate. As a consequence, $k_{d}, i^{\circ}$ was treated as an adjustable constant in the present work. The values of $k d, i^{\circ}$ used for individual simulations are justified in the Results and Discussion Section.

The temperature-programmed desorption of adsorbates was simulated using a Monte Carlo algorithm. The metal surface was represented by a 100 by 100 array of numbered sites, and periodic boundary conditions were used to eliminate edge effects. The number of metal atoms associated with an adsorbate, $n$, was chosen on the basis of information taken from the literature for a given metal-adsorbate system. Experimental observations reported in the literature were also used to determine the initial structure of the adsorbate overlayer.

The simulation of a TPD spectrum was carried out in the following fashion. Adsorbate atoms or molecules were placed on the metal surface lattice in either an ordered or random fashion to achieve a desired initial coverage, $\theta_{0}$. The temperature was initialized at To and taken to be constant at this value for the time interval $\Delta t$. A surface site (or pair of adjacent surface sites for associative desorption) was then selected in a random fashion. If the site (or both sites for associative desorption) was occupied, the 
probability of desorption was calculated using eq. 2. The local activation energy for desorption was calculated using either eq. 10 or eq. 15 , depending on whether the desorption process considered was first or second order, respectively. The calculated desorption probability was then compared with a random number, $R$, such that 0 $<R<1$. If $R<P_{i}(1)$, the adsorbate (or pair of adsorbates for associative desorption) was removed from the lattice, and $N_{d}$ in eq. 4 was incremented by one (two for associative desorption). If $R \geq$ $P_{i}(1)$, the site (or pair of sites) remained occupied. The preceding steps were repeated $\mathrm{N}_{s} \theta$ times for the case of first order desorption and $0.5 \mathrm{Ns} \theta 2$ timies for the case of second order desorption. After completing the sampling of the surface, the rate of desorption for this time interval was calculated using eq. 4.

A redistribution of the remaining adsorbates was carried out next to account for the effects of surface diffusion. A surface site and an adjacent site were chosen at random. If the surface site was occupied and the nearest-neighbor site was vacant, a probability of diffusion was calculated using eq. 5. Qi was taken as the heat of adsorption in the initial site and $Q_{j}$ was taken as the heat of adsorption in the final site. The calculated value of $P_{i j}(2)$ was then compared with a random number, $R,(0<R<1)$. If $R<P_{i j}(2)$, the adsorbate was moved from the initial site, $i$, to the adjacent site, j. Conversely, if $R \geq P(2)$, movement of the adsorbate was not allowed. A sufficient number of surface visitations was allowed to attain an equilibrium distribution of adsorbates on the surface.

The desorption and diffusion calculations described above constitute a Monte Carlo Step (MCS). As noted above, the time 
interval associated with an MCS is $\Delta t$, and over this interval the temperature is constant. At the end of an MCS the temperature was increased by the increment $\beta \Delta t$, where $\beta$ is the desired heating rate. A new MCS was then carried out at the next temperature. This process was repeated until a temperature was reached for which the surface was depleted of adsorbate. A plot of the desorption rate as a function of temperature then yielded. a TPD spectrum. In addition, the average activation energy of desorption could also be determined as a function of the total surface coverage. For both the TPD spectra and the average activation energy profiles, best-fit curves were drawn by eye through the data points. All of the calculations described above were carried out on an IBM 3090 computer. Typical run times were 2-10 CPU minutes. Random numbers were generated by the IMSL linear congruential number generator GGUBFS [20].

\subsection{RESULTS AND DISCUSSION}

\section{Comparison of Monte Carlo and Continuum Models}

Since the Monte Carlo model for TPD presented in the preceding section is new, it is useful to compare the results obtained with this model with those obtained from classical continuum representations. We begin by considering first order desorption for the case where both the activation energy and preexponential factor are constant. The values of these parameters are listed in table 1. To ensure that the probability of desorption given by eq. 2 is normalized to unity at a temperature sufficiently high to guarantee complete desorption, the time interval $\Delta t$ was determined according to: 


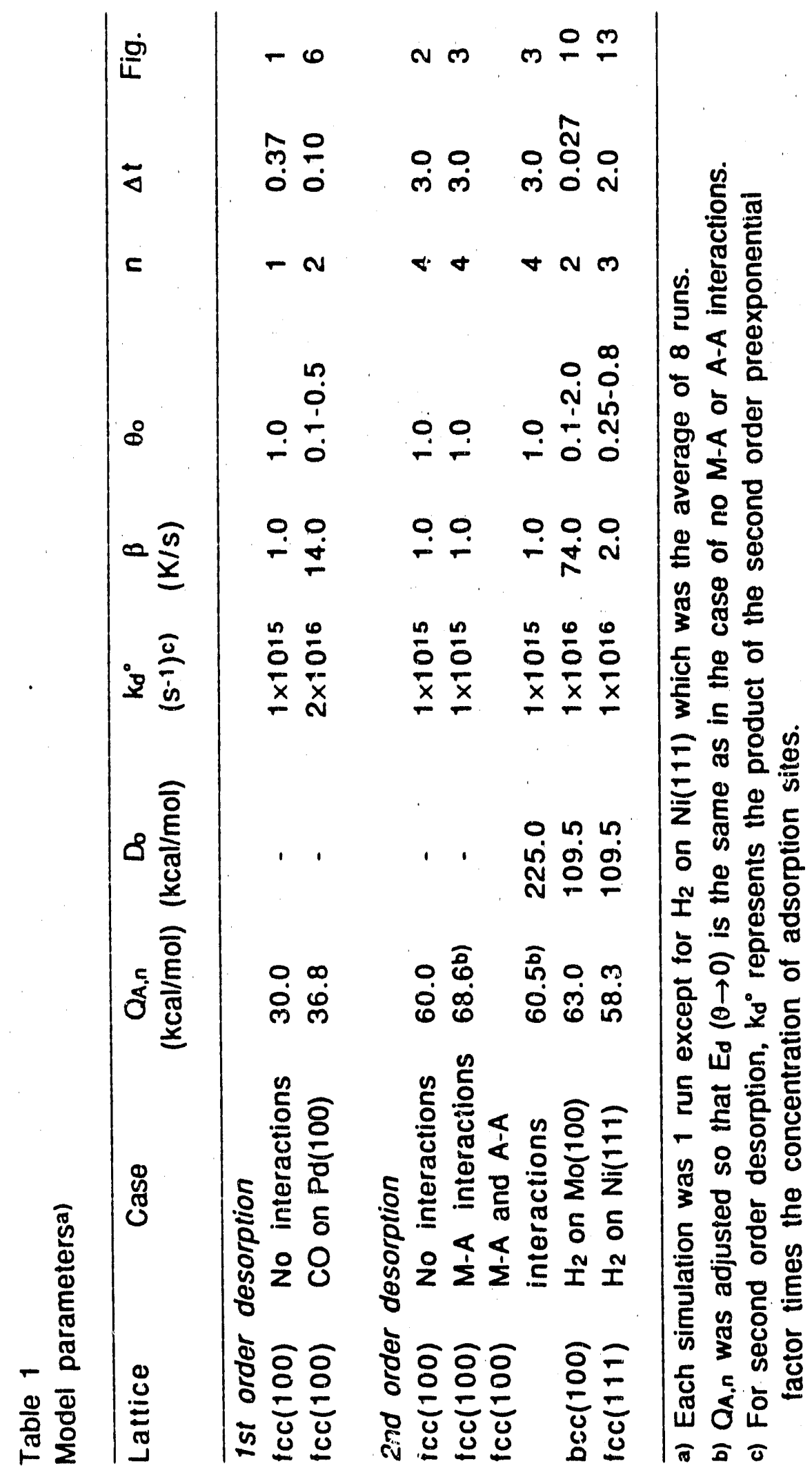


$\Delta t=\left(k_{d, 1}^{0} \exp \left[-E_{d, 1} / R T_{\max }\right]\right)^{-1}$

where $T_{\max }$ is selected to be a temperature at which the surface is depleted of adsorbate. For the case considered, Tmax was chosen to be $450 \mathrm{~K}$, and consequently $\Delta t$ is $0.37 \mathrm{~s}$. Similar reasoning for determining $\Delta t$ was used in all the simulations discussed in this paper.

Figure 1 shows that the shape and location of the TPD peak predicted by the Monte Carlo model are in good agreement with those obtained from the continuum model. The scatter in the Monte Carlo calculations is attributable to the combined effects of finite lattice size, finite step size, and statistical random number fluctuations. As demonstrated in the Appendix, the good agreement between the numerical solution and the Monte Carlo simulation is due to the equivalence of the probabilistic and continuum descriptions of desorption.

A comparison between the continuum and Monte Carlo models was also made for second order desorption kinetics with a constant activation energy. For the Monte Carlo simulation, the atomic adsorbates were allowed to diffuse on a fcc(100) lattice with probabilities of diffusion calculated using eq. 5. Only nearestneighbor species were considered as desorption partners. The parameters used are listed in table 1 and the results are shown in fig. 2. While the Monte Carlo simulation shows some scatter, it agrees well with the solution obtained from the continuum model. When a similar simulation was conducted in the absence of diffusion, a residual coverage of $9 \%$ of a monolayer remained on the surface at $T_{\max }$ in the form of isolated adsorbates with no nearest 


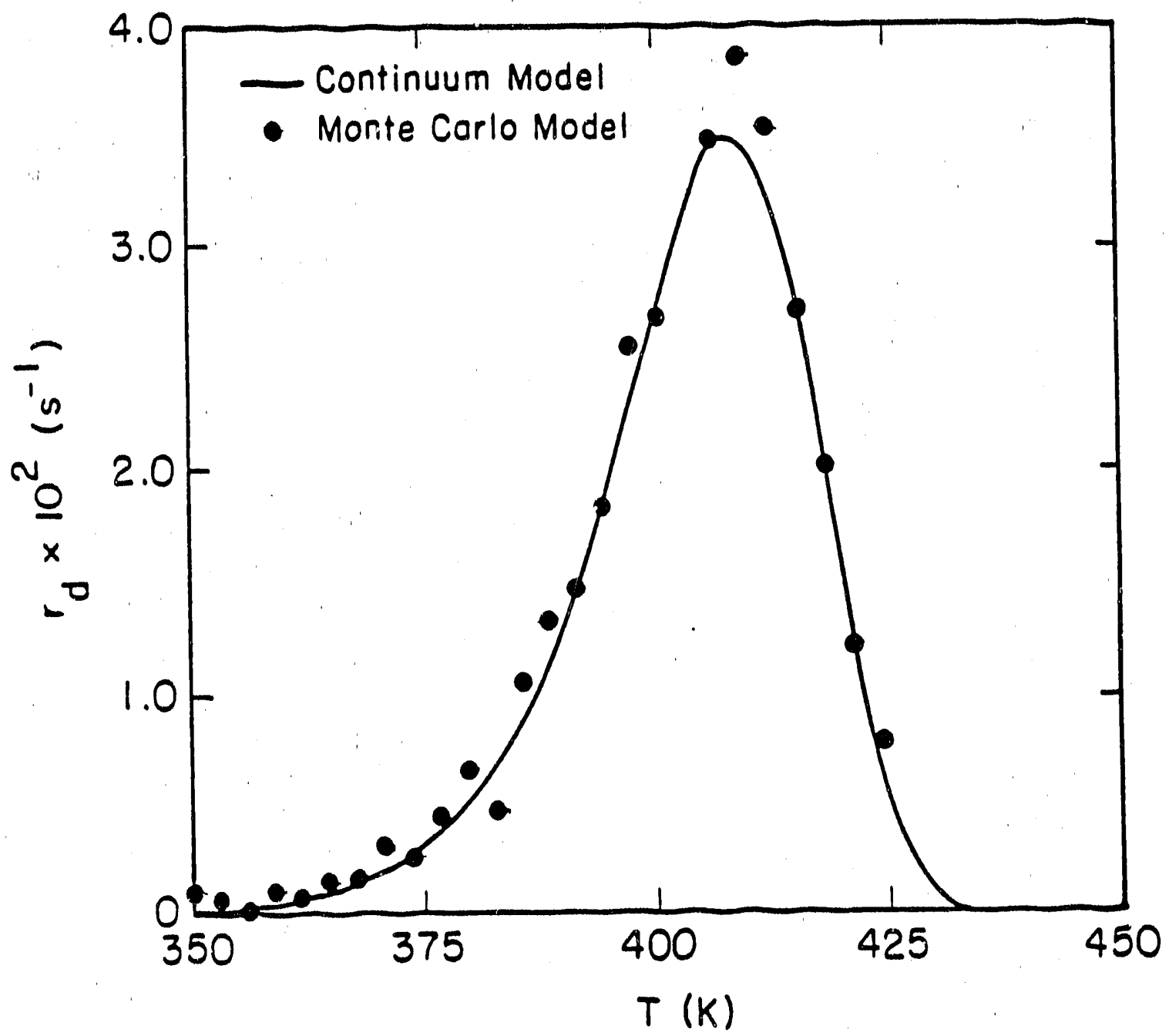

Fig. 1 Comparison of Monte Carlo and continuum models for the simulation of first order desorption where $E_{d}$ is constant. (See Table 1 for parameter values). 


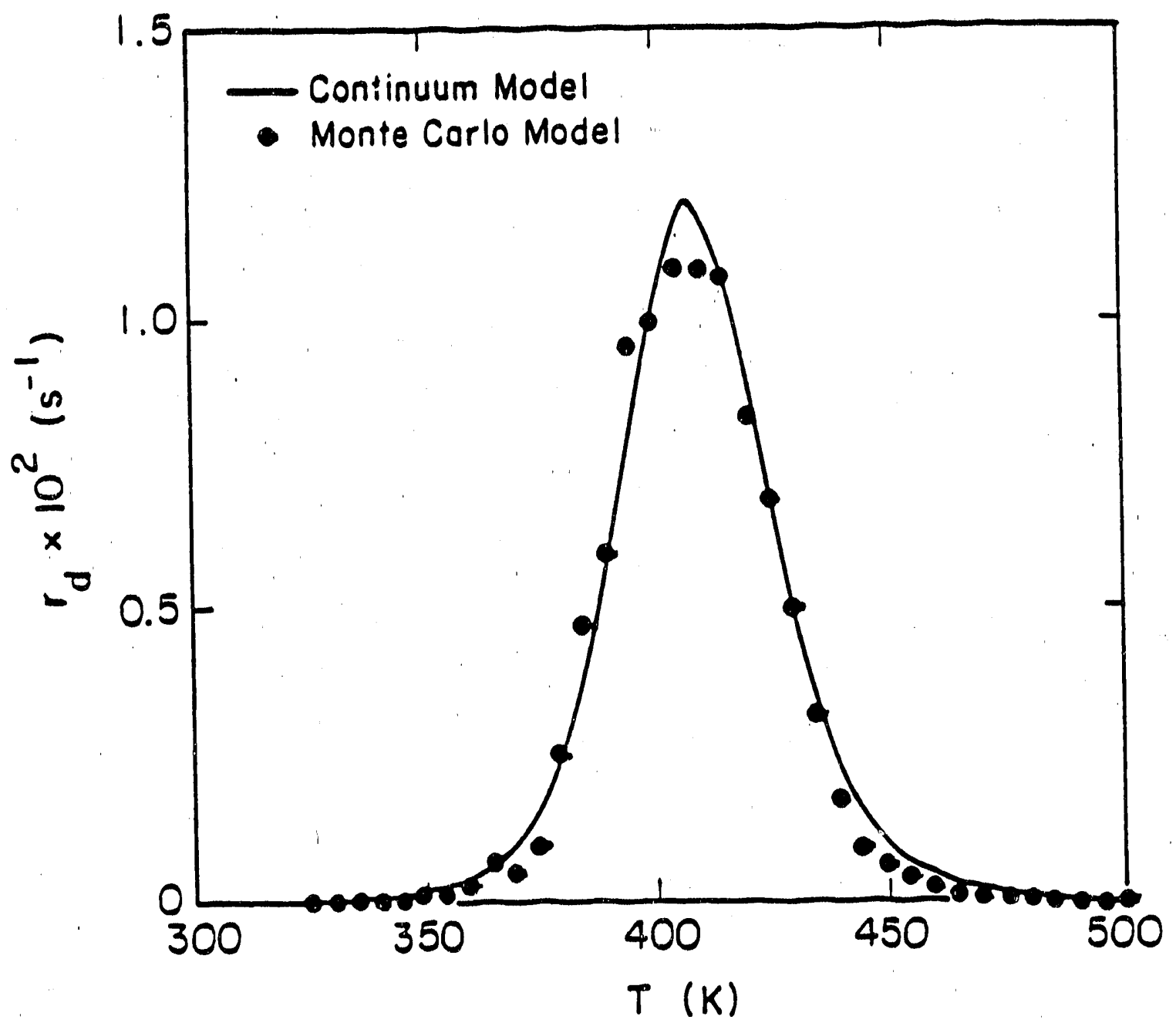

Fig. 2 Comparison of Monte Carlo and continuum models for the simulation of second order desorption where $E_{d}$ is constant. (See Table 1 for parameter values). 
neighbors. The consequence of this residual coverage on the spectrum shape was an abrupt decrease in the desorption rate at 430 $\mathrm{K}$.

The second order case was next extended to include metaladsorbate $(M-A)$ interactions. The desorption activation energy for a pair of adjacent adsorbates was calculated using eqs. 10 and 15.

The resulting TPD spectrum, shown in fig. 3, exhibits peaks at $201 \mathrm{~K}$, $255 \mathrm{~K}$, and $350 \mathrm{~K}$ in marked contrast to the single peak observed at $406 \mathrm{~K}$ for the case of constant activation energy. The three peaks. arise from the non-linear manner in which the average activation energy for the desorbing species varies with coverage. As shown in fig. 4 , the activation energy for desorbing pairs of atoms decreases with increasing increasing adsorbate coverage, the decreases being most pronounced at coverages of $0.25,0.50$, and 0.75 . The coverages at which rapid changes in the activation energy occur reflect the (100) lattice geometry chosen for this example. Figure 4 also shows the coverage dependence of the average activation energy for all pairs of adsorbates. This curve lies above the curve for the desorbing species, which indicates that at a given coverage, the desorbing atoms are, on the average, less strongly bound to the surface than those which remain adsorbed.

A comparison of the activation energy profile with the TPD spectrum shows that the flat regions of the activation energy curve correspond to changes in coverage associated with the peaks while the steep regions correspond to rearrangement of the surface by diffusion and stabilization of the adsorbates. It should also be noted that at the highest temperature shown, 0.22 monolayer of adsorbate 


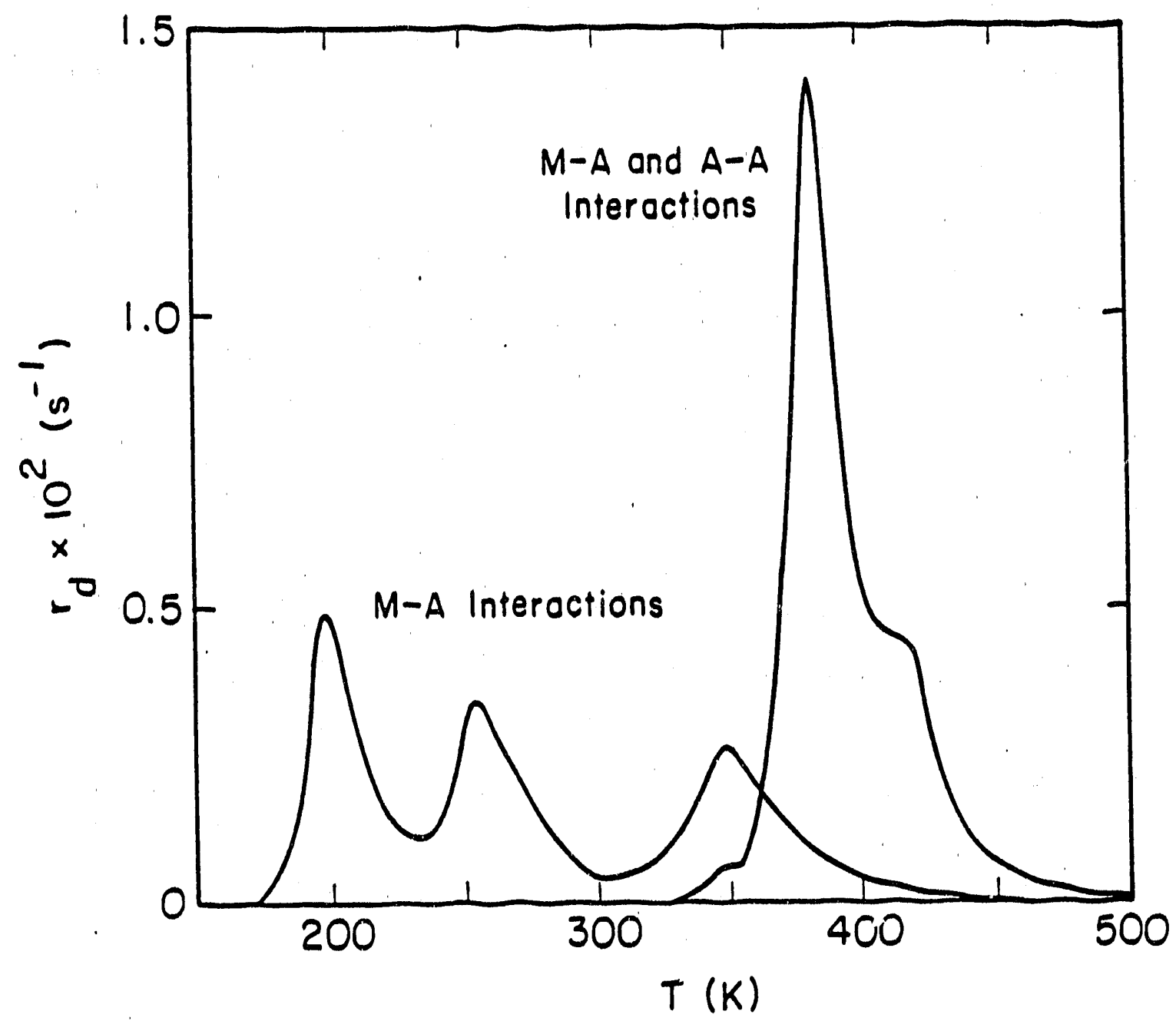

Fig. 3 Illustrations of the effects of $M-A$ and $M-A$ plus $A-A$ interactions on the TPD spectra for second order desorption. (See Table 1 for parameter values). 


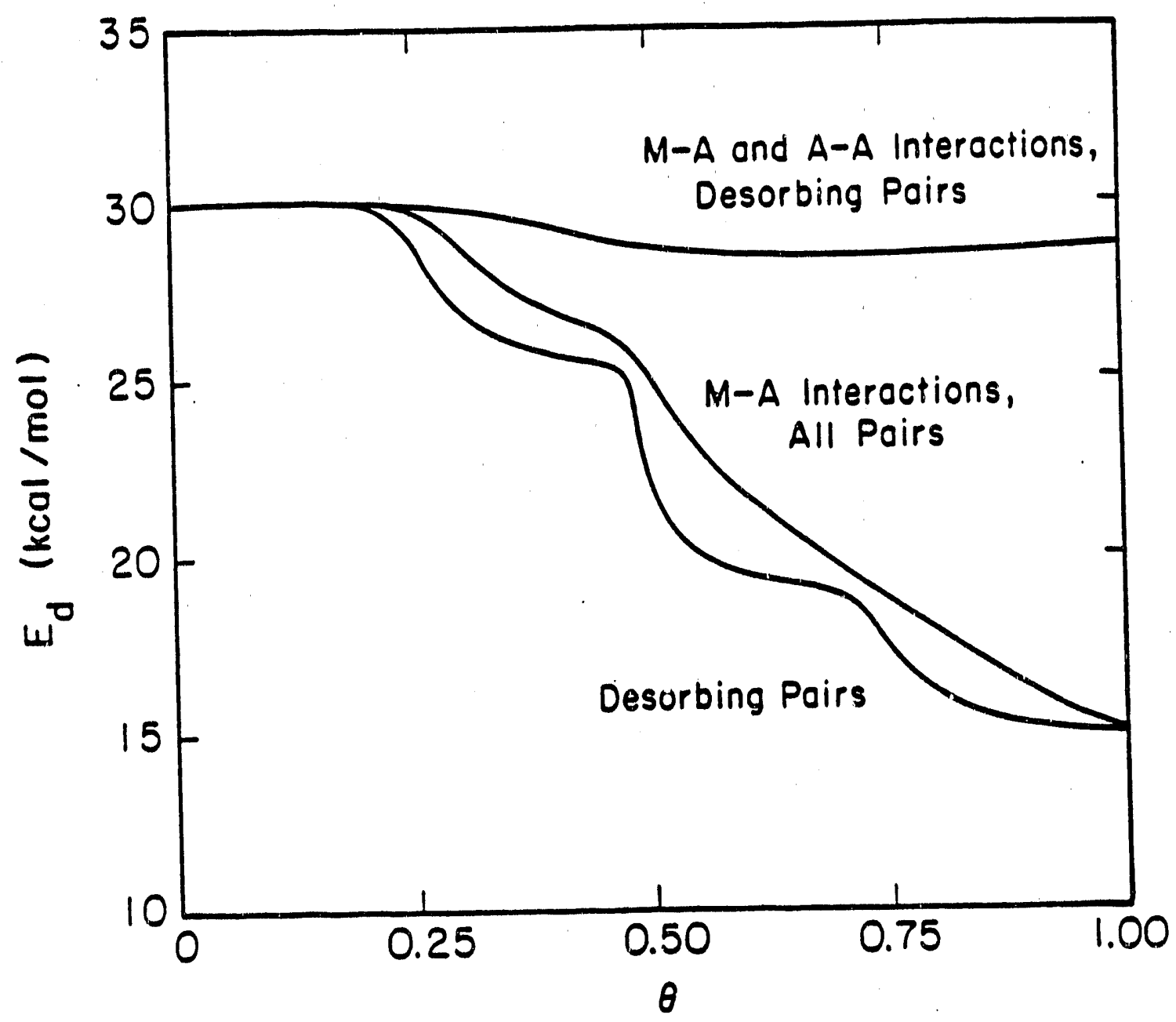

Fig. 4 Illustrations of the effects of $M-A$ and $M-A$ plus $A-A$ interactions on the average $E_{d}$ for second order desorption. 
remained on the surface in the form of isolated atoms. This situation arises because the heat of adsorption of an isolated atom is higher than that for an atom located adjacent to another atom, and as a consequence, an isolated adsorbate is unlikely to diffuse to a lattice location where it can form an A-A pair.

Finally, second order desorption was considered for the case were both metal-adsorbate (M-A) and direct adsorbate-adsorbate ( $A$ A) interactions occur. Activation energies for desorption in this case were calculated from eqs. 11 and 15. The results are shown in fig. 3. A single peak is observed at $384 \mathrm{~K}$ with a sinall low temperature shoulder at $350 \mathrm{~K}$ and a high temperature shoulder at $415 \mathrm{~K}$. The large difference between this TPD spectrum and that corresponding to the case for M-A interactions alone arises from the stabilizing influence of the attractive A-A interactions. As seen from the activation energy profile in fig. 4 , the high coverage activation energy is $12 \mathrm{kcal} / \mathrm{mol}$ greater than that for the case where only $M-A$ interactions are considered. Consequently, the peak shifts to a higher temperature and is intermediate in shape and location between the cases for $\mathrm{M}-\mathrm{A}$ interactions and no interaction (i.e. constant $E_{d}$ ). The shoulders which are observed arise from the non-linear variation of the activation energy with adsorbate coverage. When A-A interactions are taken into account, the residual coverage at $500 \mathrm{~K}$ decreases to 0.03 of a monolayer. This low residual coverage is a consequence of the attractive nature of the A-A interactions which makes diffusion of an $A$ atom to an adjacent lattice site favorable energetically.

To illustrate further the effects of $M-A$ and $A-A$ interactions, 
determinations were made of the distribution of local coverages for a fixed average coverage. Figure 5 illustrates the fraction of adsorbate pairs having 1 to 8 nearest- and/or next-nearest neighbors surrounding one member of the pair. Distributions are shown for the desorbing atoms and for all atoms residing on the surface at a given average coverage. Three cases are considered: no $M-A$ or $A-A$ interactions; $M-A$ interactions; and $M-A$ plus $A-A$ interactions. It is evident that the distribution of local coordination viumbers is sensitive to the presence or absence of $M-A$ and $A-A$ interactions. $M-A$ interactions cause a narrowing in the distribution whereas $A-A$ interactions have the opposite effect. $M-A$ interactions, which are repulsive in nature, skew the distributions to lower coordination numbers. When $M-A$ and $A-A$ interactions occur, the complex tradeoff between repulsive $M-A$. and attractive $A$ $A$ efiects skews the distribution to high coordination numbers at high coverage $(\theta>0.5)$ and to low coordination numbers at low coverage $(\theta<0.5)$. In the absence of $M-A$ and $A-A$ interactions the distribution of coordination numbers for all pairs, shown in fig. 5 , is in excellent agreement with the relationship $f(C N+1)=\frac{7 !}{C N !(7-C N) !} \theta^{C N}(1-\theta)^{7-C N}$, where $0 \leq C N \leq 1$

derived for a random placement of adsorbate atoms on the surface sites.

CO Desorption from Pd(100)

The structure of carbon monoxide adsorbed on the (100) plane of palladium is well established. Early investigations have 


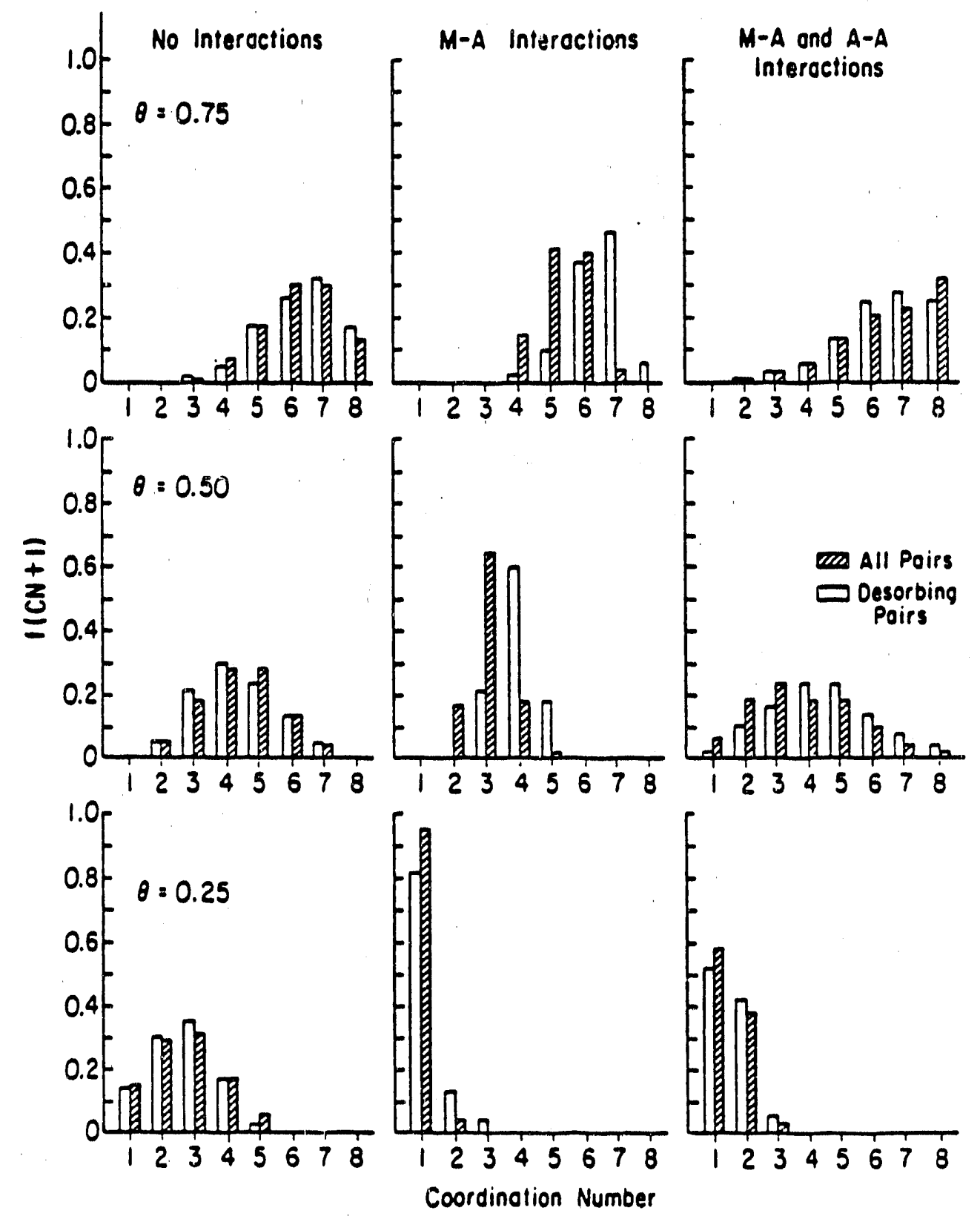

Fig. 5 Illustrations of the effects of $M-A$ and $M-A$ plus $A-A$ interactions on $f(C N+1)$ for second order desorption. 
demonstrated that $\mathrm{CO}$ adsorption occurs molecularly and is completely reversible [21,22]. LEED analysis indicates that the CO molecules occupy both types of bridge sites and form a $c(2 \sqrt{2} \times$ $\sqrt{2}$ R45 overlayer at one-half of a monolayer [21-24]. Vibrational spectroscopic measurements by EELS and IR further support the bridge-bonding coordination for all coverages up to one-half monolayer [24,25]. Analysis of equilibrium isosteres and isobars has shown that the isosteric heat of adsorption (which is approximately equal to the activation energy for desorption in the case of nonactivated adsorption) for $\mathrm{CO}$ is $38.5 \mathrm{kcal} / \mathrm{mol}$ at low coverages and decreases with increasing coverage [22,23]. The activation energy for desorption and the preexponential factor are reported to be $36.8 \mathrm{kcal} / \mathrm{mol}$ and $2 \AA 1016 \mathrm{~s}^{-1}$, respectively, in the limit of low coverage [23].

Simulation of $\mathrm{CO}$ desorption from a $\mathrm{Pd}(100)$ surface was conducted in the following manner. A carbon monoxide coverage of one-half was placed on a $\mathrm{Pd}(100)$ lattice in a $c(2 \sqrt{ } 2 \times \sqrt{2}) \mathrm{R} 45$ overlayer. The activation energy and the preexponential factor at $z \in r o$ coverage were taken from the experimental results presented in ref. [23] and are listed in table 1. The activation energy as a function of local CO coverage was calculated from eq. 10. Carbon monoxide molecules were allowed to diffuse on the surface with probabilities for individual diffusion jumps calculated from eq. 5 . Because of steric constraints, a maximum of two $\mathrm{CO}$ molecules was allowed to be bridge bonded to a single Pd metal atom; furthermore, the adsorbates had to be located 180 degrees from each other on a metal atom. Exclusion of adsorbates bonded 90 degrees to 
each other seems reasonable as this distance on the Pd surface is $1.94 A$ and the CO hard sphere diameter is approximately $2.7 \AA$ [22].

Results of simulations for coverages equal to and less than 0.5 are shown in fig. 6. The spectra exhibit first order desorption kinetics with a peak temperature at $490 \mathrm{~K}$. Some low temperature broadening of the desorption peak is evident at the highest coverages. The extent of the low temperature broadening predicted by the simulation is not as large as is observed experimentally (see fig. 7). This is due most likely to the use of a constant preexponential factor in the simulations. Interpretation of the experimental data using a continuum model [23] led to the conclusion that the preexponential factor increases steeply with increasing coverage for coverages greater than 0.4 . This variation in the preexponential factor was not included in the simulations because it could not be predicted on the basis of local environment without introducing an arbitrary parameter.

Values of the average activation energy for desorption as a function of coverage are shown in fig. 8. The activation energy exhibits a relatively constant value until a coverage of 0.40 at which point it begins to decrease sharply. Also shown in fig. 8 are values for the activation energy deduced from experimentally determined isobars [23]. It is evident that the coverage dependence of the activation energy determined from the Monte Carlo simulation is roughly similar to that observed experimentally.

$\mathrm{H}_{2}$ Desorption from Mo(100)

Hydrogen adsorbed on the (100) plane of molybdenum exhibits 


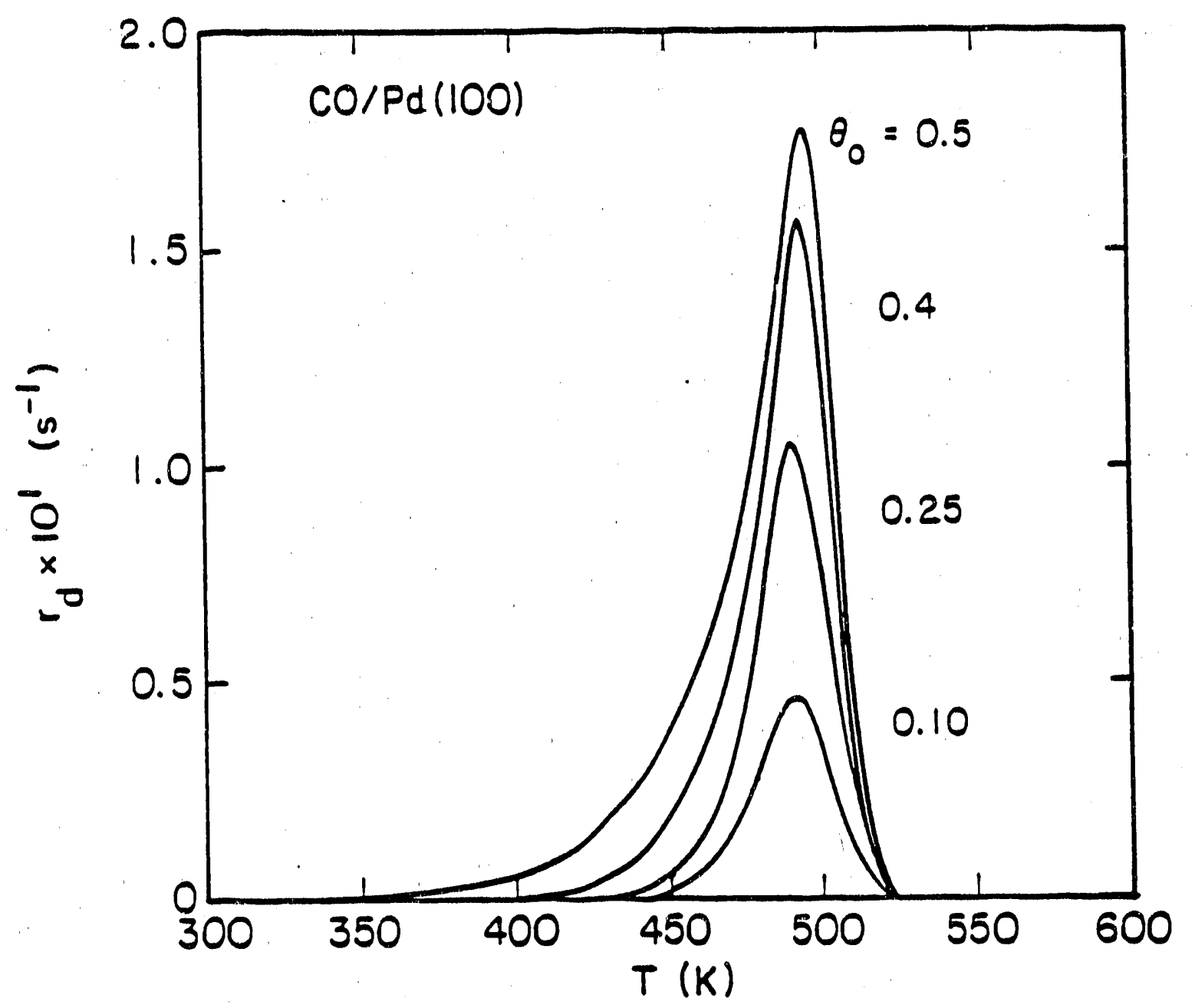

Fig. 6 Monte Carlo simulations of CO desorption from $\mathrm{Pd}(100)$. (See Table 1 for parameter values). 
182

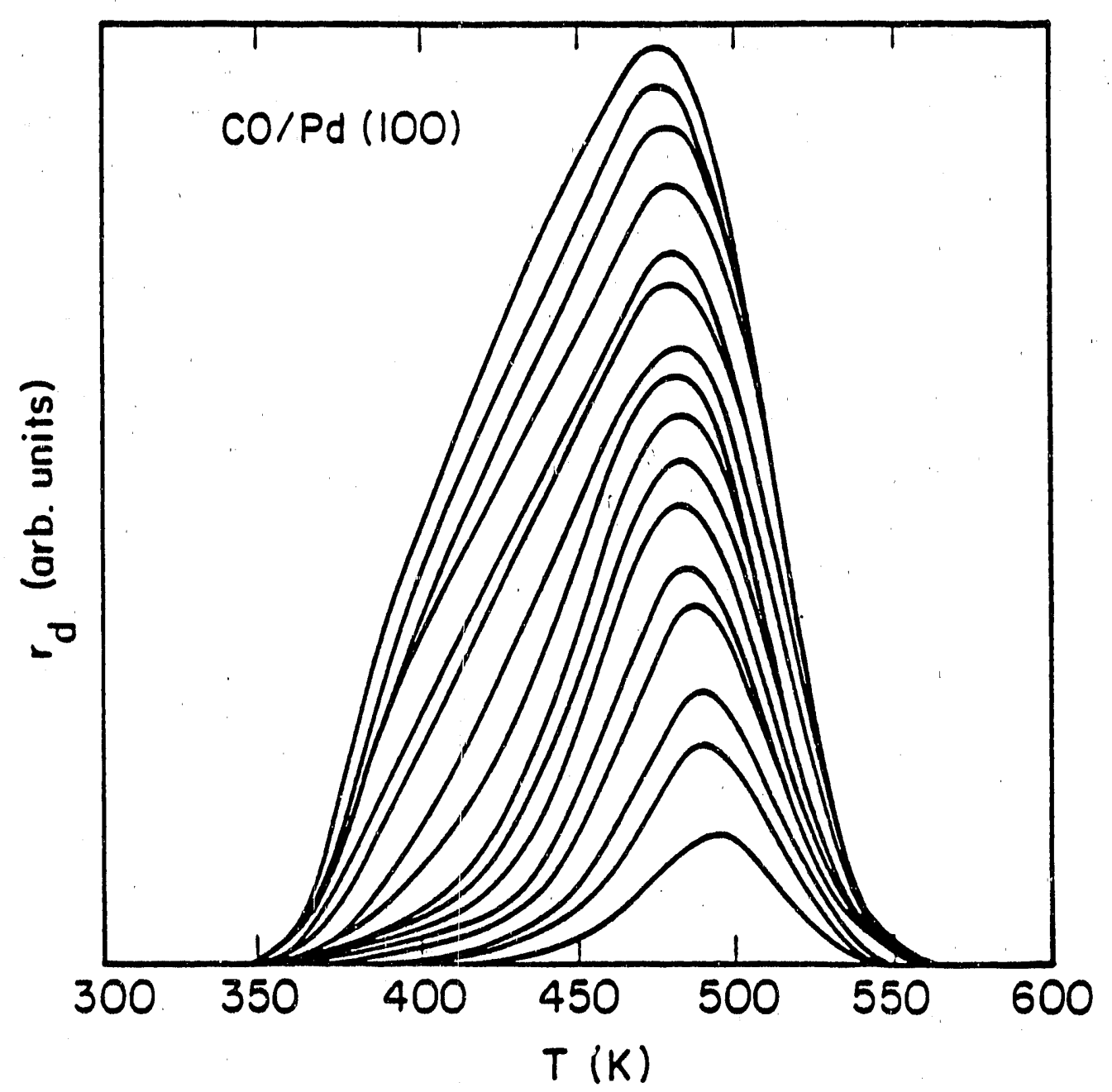

Fig. 7 TPD spectra for CO desorption from Pd(100) [23]. 


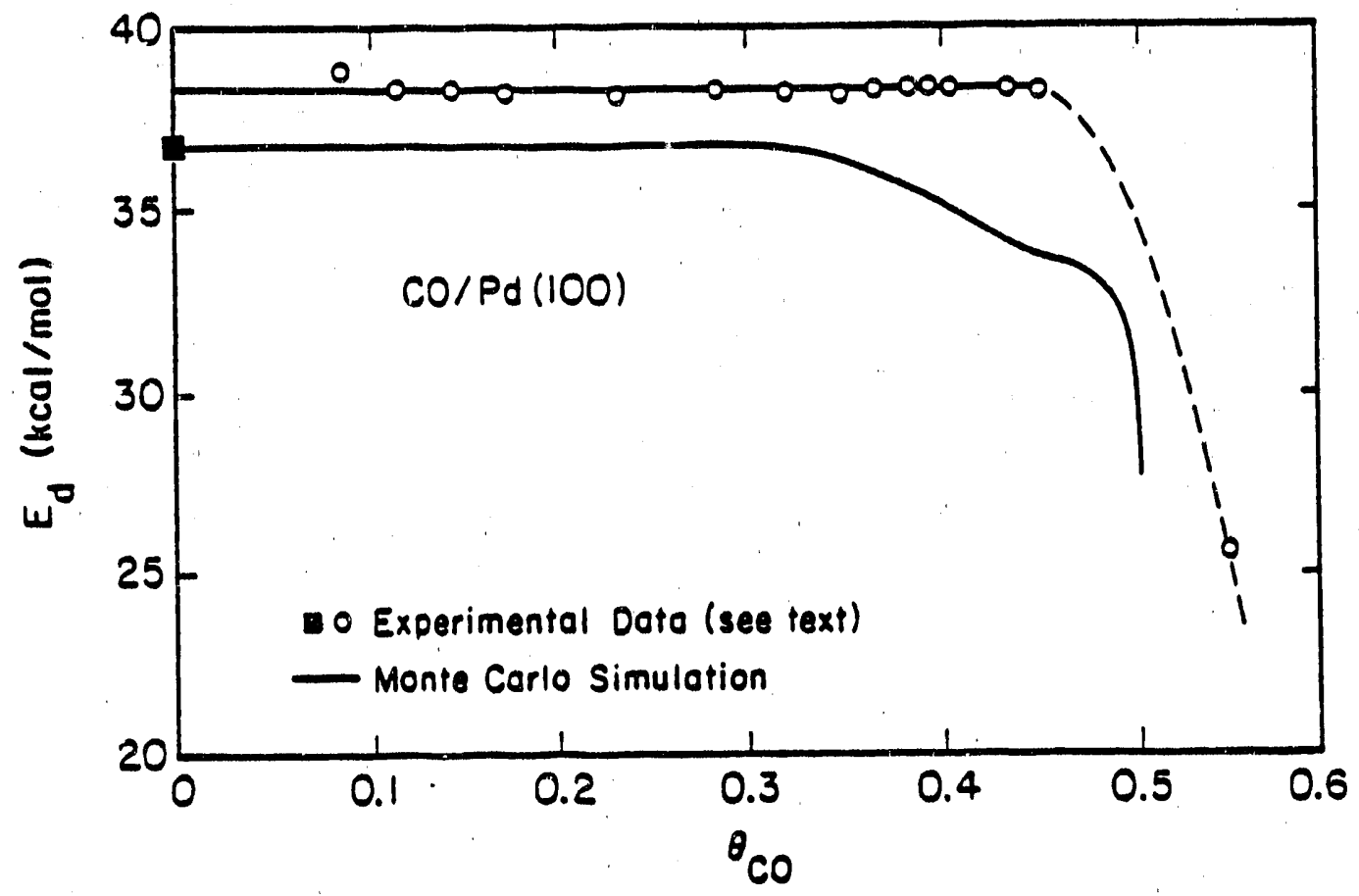

Fig. 8 Variation of tha average $E_{d}$ with $\theta c o$ for $C O$ desorption from Pd(100). 
TPD spectra such as those shown in fig. 9 [26]. From hydrogen isotope experiments, it is now agreed that the three states, which occur in 2:1:1 ratio, all arise from dissociatively adsorbed hydrogen and that saturation ceverage corresponds to two $[26,27]$. Vibrational spectroscopy suggests that the atomic hydrogen is located in bridge sites but that the nature of these bridge sites changes with coverage [27-29]. It is also believed that the molybdenum surface undergoes a surface reconstruction which consists of periodic displacements of molybdenum atoms by approximately $0.2 \AA$ from their unreconstructed bcc location [27-33]. Although not fully characterized, this reconstruction occurs primarily at temperatures below $300 \mathrm{~K}$ and at low adsorbate coverages [27-33].

Simulations were conducted by assigning hydrogen atoms to bridge sites at all coverages. Consideration of atomic hydrogen distribution on the surface required including both nearest- and nextnearest-neighbor hydrogen atoms as potential desorption partners. Values of the activation energy and the preexponential factor used to initialize the model were obtained from a Redhead analysis of the low coverage experimental spectra shown in fig. 9 and are listed in table 1. No attempt was made to account for the effect of molybdenum displacements due to the surface reconstruction. Direct $\mathrm{H} \cdot \mathrm{H}$ interactions were limited to nearest-neighbor hydrogen atoms and accounted for in the following manner. The bond order for $\mathrm{H}-\mathrm{H}$ interactions can, in principle, be calculated from eq. 7 . It is known, though, that at long $\mathrm{H}-\mathrm{H}$ distances, the Morse potential is a poor approximation to the bond dissociation characteristics. To 


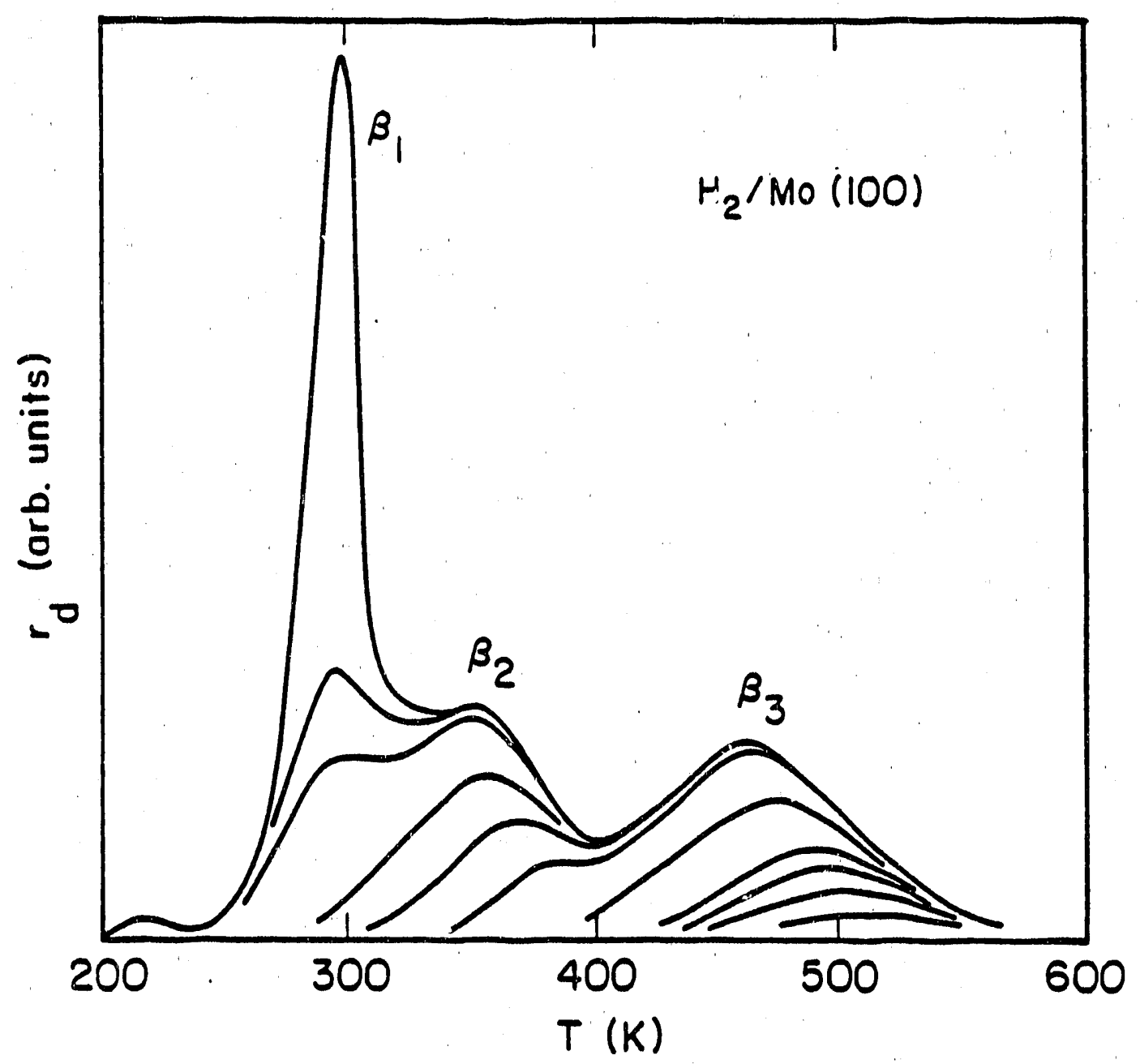

Fig. 9 TPD spectra for $\mathrm{H}_{2}$ desorption from $\mathrm{Mo}(100)$ [26]. 
obtain better agreement with experimental data for gaseous hydrogen, the Morse potential parameter a in eq. 7 can be replaced by $a^{\prime}$ which is a three parameter function of distance given by: $a^{\prime}=\left\{a_{0}\left[1+\gamma\left(r-r_{0}\right)+\lambda\left(r-r_{0}\right)^{2}\right]\right\}^{-1}$

Values of the parameters ao, $\gamma$ and $\lambda$ in eq. 18 were taken from ref. [34]. The activation energy for $\mathrm{H}_{2}$ desorption was then calculated as a function of coverage using eqs. 11 and 15.

Results of TPD simulations for different initial coverages randomly distributed on the surface are shown in fig. 10. Three peaks are observed at 285,381 , and $459 \mathrm{~K}$. These features are referred to as $\beta_{1}, \beta_{2}$, and $\beta_{3}$ and have areas which are in the ratio of $2: 1: 1$. As the initial coverage increases, first the $\beta_{3}$, then the $\beta_{2}$, and finally the $\beta_{1}$ peak is populated. Many features of the simulated spectra agree quite well with those of the experimentally observed spectra shown in fig. 9. It is noted that the predicted locations of the $\beta_{1}$ and $\beta_{3}$ peaks are almost identical to those observed experimentally, and that the ratios of all three peak areas are identical to those found experimentally. Figure 10 does show, however, that the predicted position of the $\beta_{2}$ peak is $30 \mathrm{~K}$ higher than that found experimentally (see fig. 9). Nevertheless, the level of agreement between the spectra shown in figs. 9 and 10 is sufficiently high to suggest that the three $\beta$ peaks do not arise from three distinct types of the sites but rather from the non-linear manner in which the activation energy varies with coverage.

The variation in the activation energy with coverage predicted from the Monte Carlo model is shown in fig. 11. The activation 


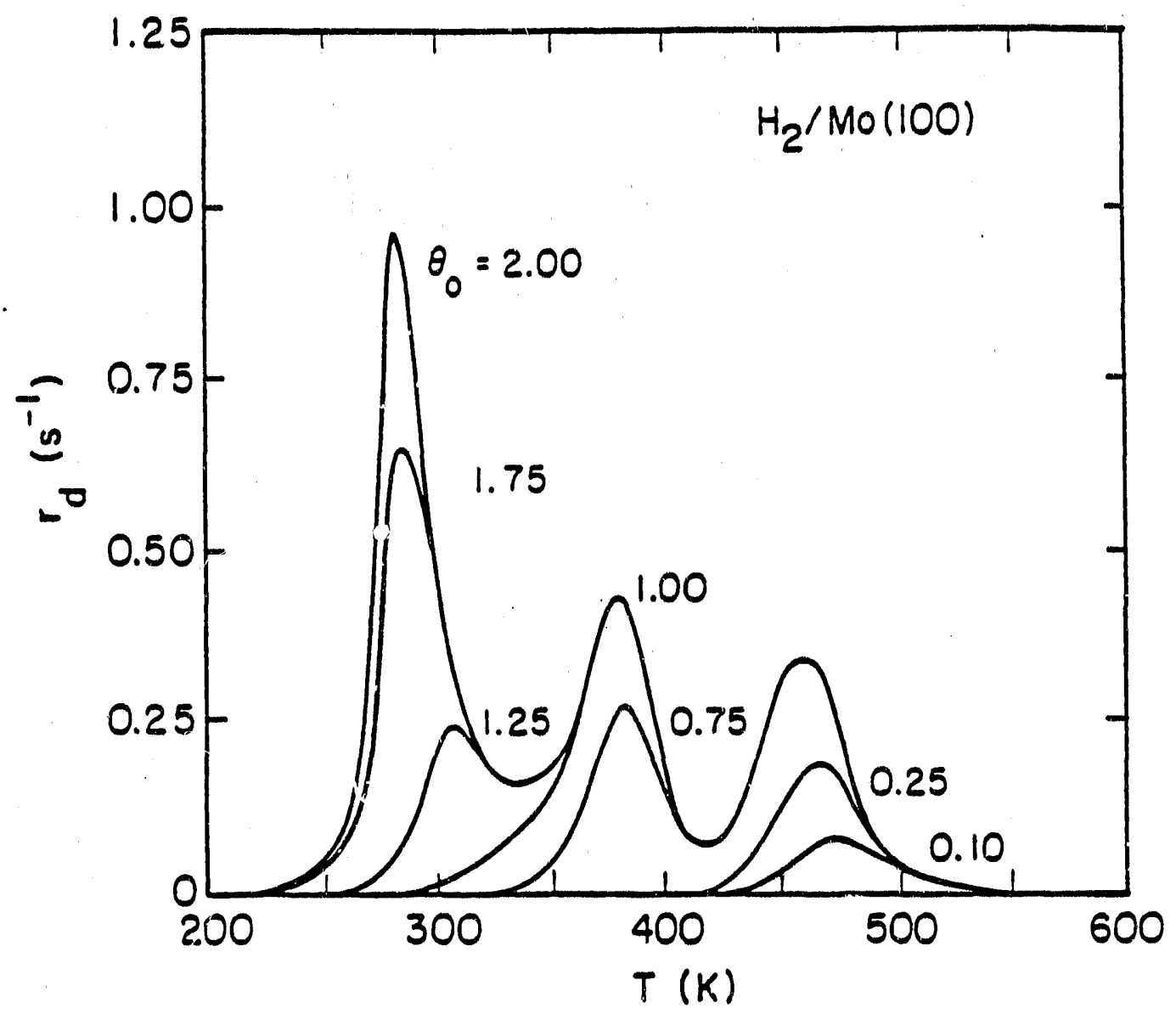

Fig. 10 Monte Carlo simulations of $\mathrm{H}_{2}$ desorption from $\mathrm{Mo}(100)$. (See Table 1 for parameter values). 
188

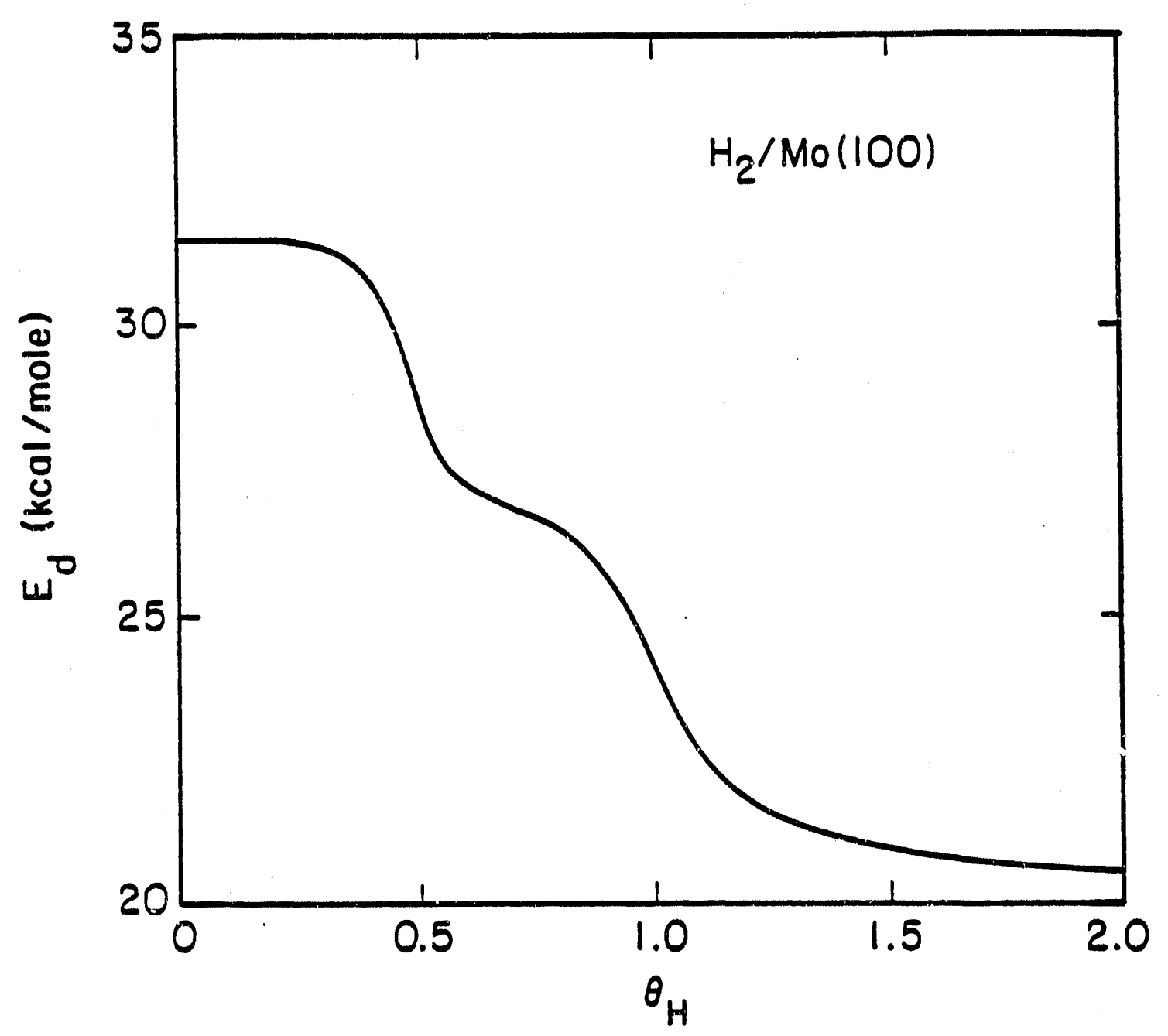

Fig. 11 Variation of the average $E_{d}$ with $\theta_{H}$ for $\mathrm{H}_{2}$ desorption from Mo(100). 
energy is relatively constant up to a coverage of 0.5 at which point the activation energy decreases rapidly to a new plateau. The second plateau is maintained up to a coverage of about 1.0.

Thereafter, the activation energy steadily decreases down to its minimum value at a coverage of 2.0. The pattern shown in fig. 11 closely resembles that observed experimentally [35].

\section{$\mathrm{H}_{2}$ Desorption from $\mathrm{Ni}(111)$}

Desorption of $\mathrm{H}_{2}$ from the (111) plane of $\mathrm{Ni}$ produces TPD spectra such as those shown in fig. 12. Both sets of experimental data exhibit two peaks with maxima separated by $30-80 \mathrm{~K}[36,37]$. Isotopic labelling experiments have shown that the two peaks arise from dissociatively adsorbed hydrogen [36-38]. Unlike Mo(100), there is no evidence for reconstruction of the Ni(111) surface [36]. Dynamic LEED IV analysis [36] and EELS [39] indicate that hydrogen occupies both the fCc and hcp three-fold hollows. LEED analysis further suggests that at temperatures above $270 \mathrm{~K}$, ordering of the hydrogen overlayer does not occur and that island formation is unlikely [36]. The maximum coverage observed is $\theta=0.8 \pm 0.2$. This is less than a coverage of 2.0 which would correspond to full occupation of both types of three-fold sites [36].

Simulations were conducted by assigning hydrogen atoms to both types of three-fold sites at all coverages. Occupation of adjacent fcc and hcp type hollows was not allowed based on the LEED observations mentioned previously [36]. The preexponential factor and the activation energy at zero coverage were obtained from a Redhead analysis of the TPD spectra reported in ref. [37]. 

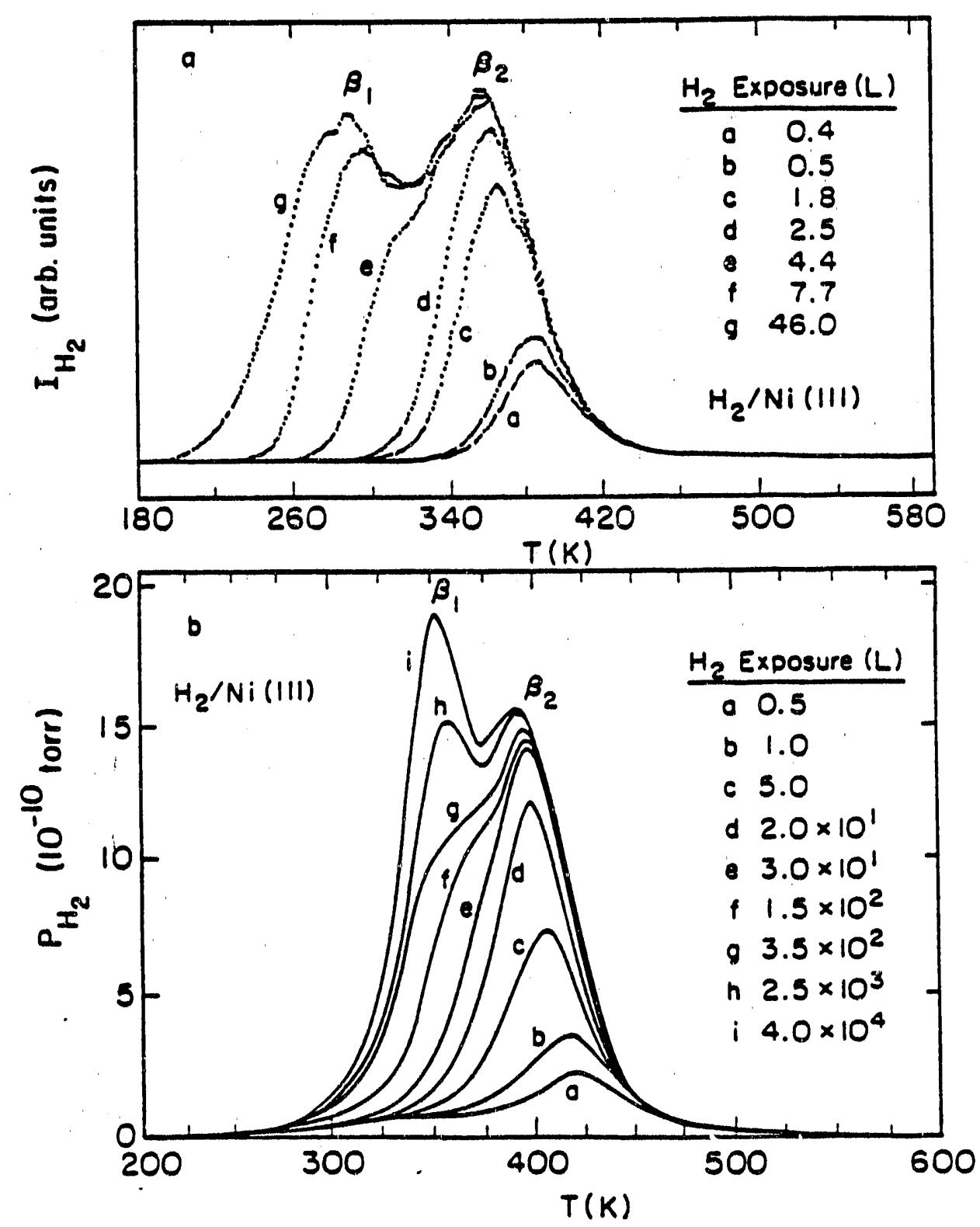

Fig. 12 TPD spectra for $\mathrm{H}_{2}$ desorption from $\mathrm{Ni}(111)$. Panel a is from ref. [37] and panel $b$ is from ref. [36]. 
The results of simulations for different initial coverages are shown in fig. 13 and the variation of $E_{d}$ for desorbing $\mathrm{H}_{2}$ molecules with coverage is given in fig. 14. Figure 13 shows that for $\theta_{0}=0.80$, three overlapping peaks occur at 275,300 , and $340 \mathrm{~K}$. The first two features fall in the range of temperatures for the $\beta_{1}$ peak seen in fig. 12a, whereas the third feature coincides with the $\beta_{2}$ peak of fig. 12a. Careful inspection of fig. 12 a suggests that the peak designated $\beta_{1}$ may in fact be comprised of two components. A similar conclusion can be drawn from the TPD spectrum for $D_{2}$ desorption presented in ref. [37]. It should be noted that the relative peak heights in the spectrum computed for $\theta_{0}=0.80$, seen in fig. 13 , differ from those shown in fig. 12a, and are closer to those shown in fig. 12b. The reason for this is that an initial coverage of 0.8 may be greater than the highest initial coverage that could be achieved by an $\mathrm{H}_{2}$ exposure of $46 \mathrm{~L}$. This is certainly consistent with the observation that as the maximum exposure is increased, the $\beta_{1}$ feature becomes more intense than the $\beta_{2}$ feature (see fig. 12b).

\subsection{CONCLUSIONS}

A Monte Carlo model has been developed to describe temperature-programmed desorption of adsorbates from single crystal surfaces. The model accounts for the dependence of the activation energy for desorption on metal-adsorbate $(M-A)$ and adsorbate-adsorbate (A-A) interactions and for the diffusion of adsorbates over the metal surface. The model correctly predicts not only the number and location of the peaks in a TPD spectrum, but also the dependence of the activation energy for desorption on the 
192

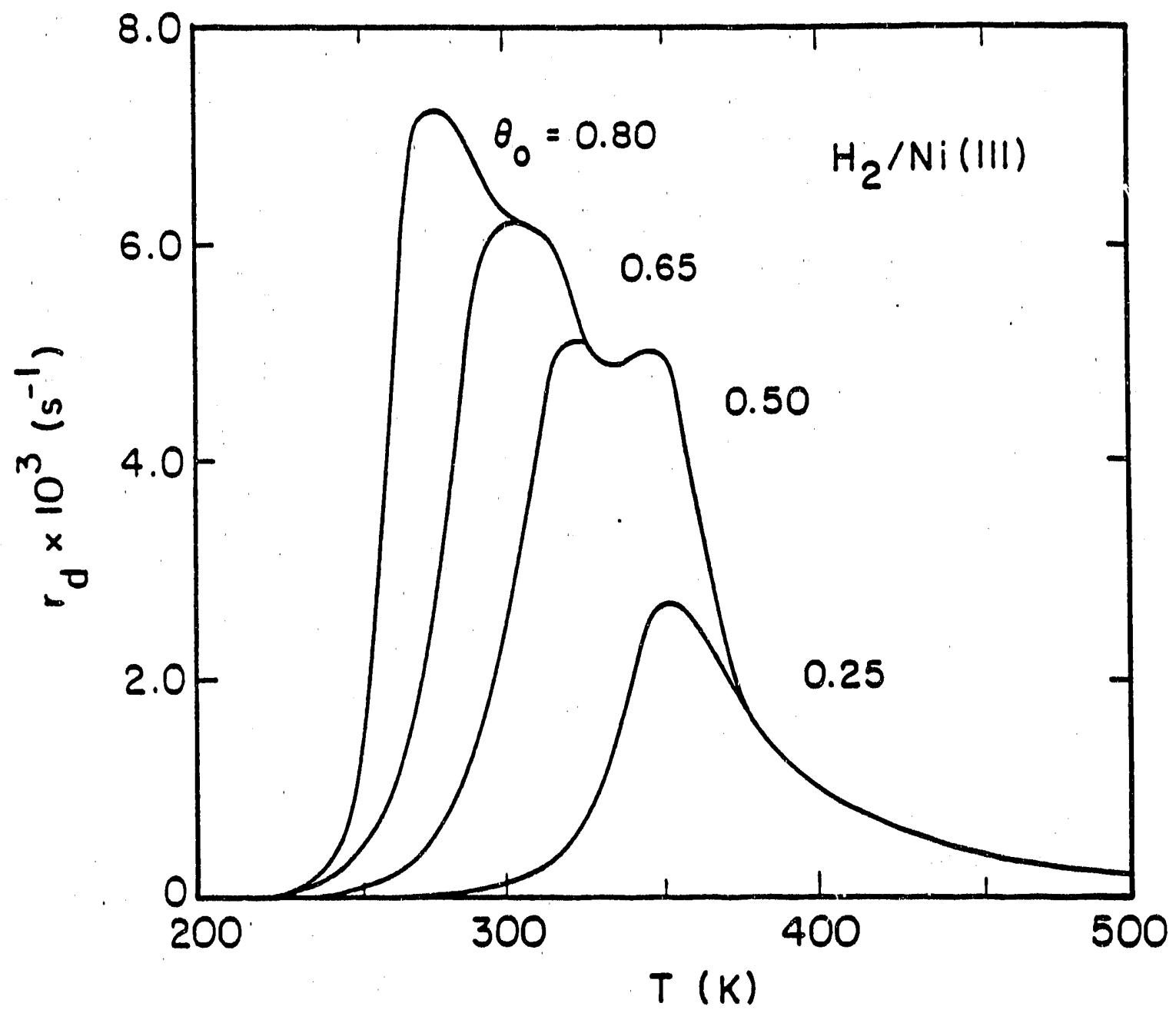

Fig. 13 Monte Carlo simulations of $\mathrm{H}_{2}$ desorption from $\mathrm{Ni}(111)$. '(See Table 1 for parameter values). 


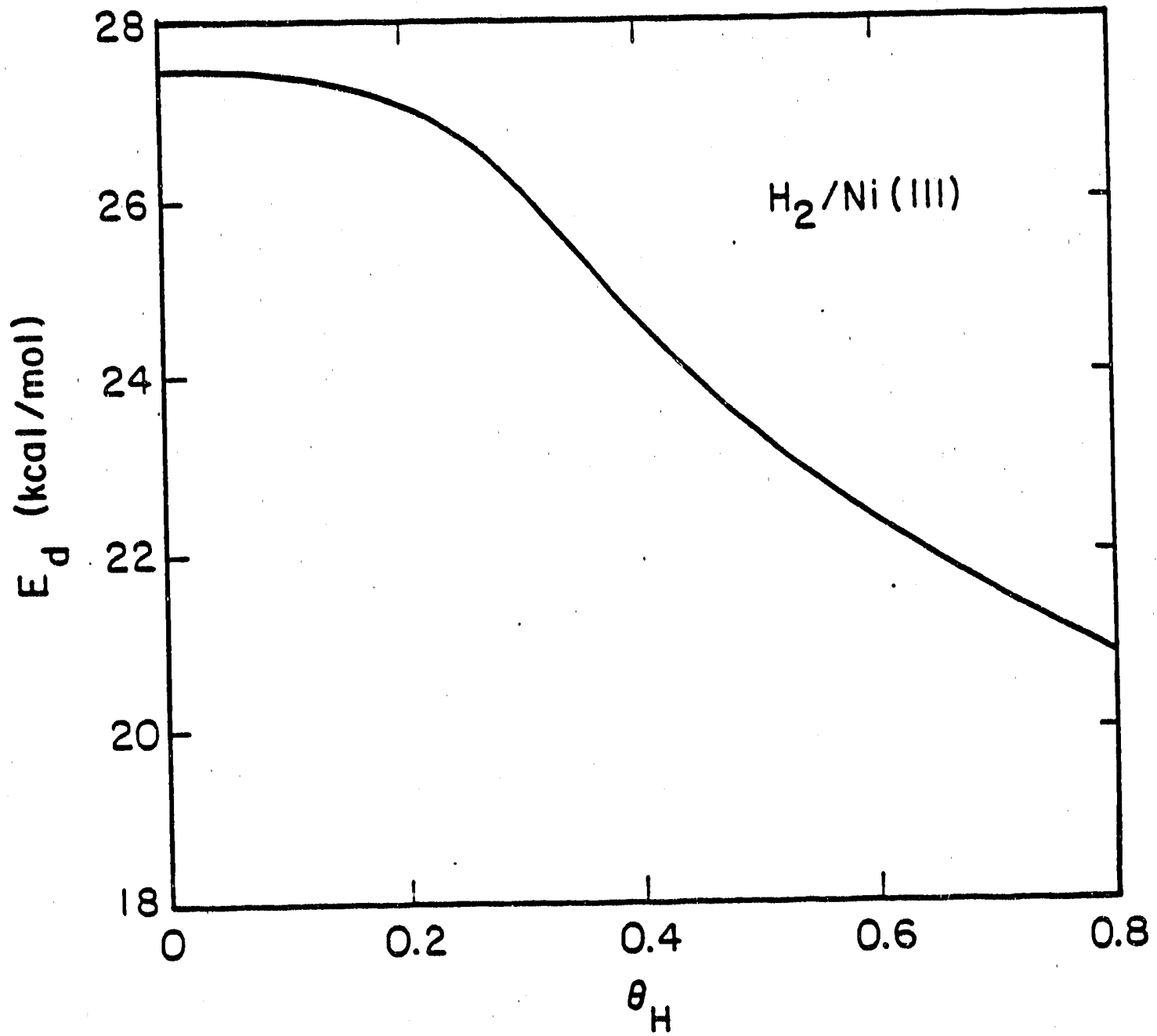

Fig. 14 Variation of the average $E_{d}$ with $\theta_{H}$ for $\mathrm{H}_{2}$ disorption from $\mathrm{Ni}(111)$. 
average surface coverage and the distribution of local coverages for a given average coverage.

The inclusion of M-A and A-A interactions in the model has a pronounced effect on the shape of the predicted TPD spectrum. Where only a single peak is observed in the absence of $M-A$ and $A-A$ interactions, multiple peaks are found when these interactions are included. Moreover, the introduction of M-A and A-A interactions causes the activation energy to decrease with increasing coverage, the shape of this function depending on the strength of these interactions. The calculations also show that the activation energy of the desorbing species is typically less than or equal to the average value for the entire adlayer. In physical terms, this means that for a given coverage, only the most weakly bound species desorb.

The method described in this paper has been used to simulate the desorption of $\mathrm{CO}$ from $\mathrm{Pd}(100)$ and the desorption of $\mathrm{H}_{2}$ from $\mathrm{Mo}(100)$ and $\mathrm{Ni}(111)$. The simulated spectra for $\mathrm{CO}$ desorption from $\operatorname{Pd}(100)$ are narrower than those observed experimentally, most likely due to the neglect of the variation in the preexponential factor with coverage. However, the predicted variation in activation energy with coverage agrees reasonably well with that found experimentally. Monte Carlo simulation of $\mathrm{H}_{2}$ desorption from $\mathrm{Mo}(100)$ and $\mathrm{Ni}(111)$ predicts three TPD peaks in both cases. The number of peaks, the peak temperatures for each peak, and the relative peak intensities are in good agreement with experimental observation. These calculations demonstrate that the appearance of multiple peaks is due to the non-linear nature of the $M-A$ and $A-A$ 
interactions, rather than to the occurrence of distinct binding states.

ACKNOWLDGMENT

The authors wish to thank Dr. E. Shustorovich of Kodak Research Laboratories for many helpful discussions of this research. This work was supported by the Director, Office of Basic Energy Sciences, Chemical Sciences Division of the U.S. Department of Energy under contract DE-AC03-76SF00098. 


\section{APPENDIX}

We derive here probabilistic rate expressions which are equivalent to the continuum rate expressions. For any $\alpha$-order kinetic rate process, the change in surface coverage can be represented by:

$d \theta_{i} / d t=k_{d, i}^{0} \exp \left[-E_{d, i} / R T\right] \theta_{i}^{\alpha}$

where subscript $i$ represents a single type of environment. The rate of desorption as observed in the gas phase is then:

$r_{d}=\frac{\theta_{i}}{\alpha d t}=\frac{1}{\alpha} k_{d, i}^{0} \exp \left[\frac{-E_{d, i}}{R T}\right] \theta_{i}^{\alpha}$

The probability of desorption in a given time interval can be expressed as

$P_{i}^{(1)}=\int_{11}^{12} k_{d,} \theta_{i}^{\alpha} d t / \int_{0} k_{d, j} \theta_{i}^{\alpha} d t$

The denominator in eq. $A 3$ is $\theta 10$, the initial coverage in the ith environment. For sufficiently small time intervals, eq. A3 can be approximated by

$P_{i}^{(1)} \approx k_{d, i} \theta_{i}^{\alpha} \Delta t / \theta_{i o}$

The number of adsorbates that have desorbed in the interval $\Delta t$ is:

$N_{d, i}=P_{i}^{(1)} N_{s} \theta_{i o}$

where $N_{s}$ is the number of adsorption sites. The turnover frequency per surface atom as observed in the gas phase is then

$r_{d, i}=N_{d, i} /\left(\alpha \Delta t N_{s}\right)$

Substitution of eqs. $A 4$ and $A 5$ into eq. $A 6$ yields eq. $A 2$ which demonstrates the equivalence between probabilistic formulations 
and the more familiar continuum rate expressions. When many adsorbate environments coexist on the surface, the total rate of desorption can be expressed as $r_{d, i}=\sum_{i} N_{d, i} /\left(\alpha \Delta t N_{s}\right)$

\section{NOMENCLATURE}
A
Adsorbate
a Morse potential constant $(A)$
a' Morse potential function $(A)$
a. Morse potential constant
ON Coordination number
DAB Bond dissociation energy for $A-B$ bond $(\mathrm{kcal} / \mathrm{mol}$ )
Ed Activation energy for desorption ( $\mathrm{kcal} / \mathrm{mol}$ )
Ed,i Activation energy for desorption from the ith environment (kcal/mol)

Ed,AA' Activation energy for desorption for a diatomic pair $A$ and $A^{\prime}$ (kcal/mol)

$f(C N+1)$ Fraction of adsorbate pairs having a given number of nearest-neighbors surrounding one member of the pair

$k d^{\circ} \quad$ Preexponential factor for desorption (s-1)

$k d, i^{\circ} \quad$ Preexponential factor for desorption from the ith environment $(s \cdot 1)$

$k_{d, i} \quad$ Rate coefficient for desorption from ith environment (s-1)

$\mathrm{ml}_{\mathrm{N}} \quad$ Number of adsorbates bonded to ith metal atom

$\mathrm{N} \quad$ Number of adsorbate atoms or molecules (mol)

$\mathrm{No}_{\mathrm{N}} \quad$ Number of adsorbate atoms or molecules desorbing (mol)

$\mathrm{Nd}, \mathrm{i} \quad$ Number of adsorbate atoms or molecules desorbing from the ith environment (mol)

$\mathrm{Ns}_{\mathrm{N}} \quad$ Number of surface metal atoms (mol)

$n \quad$ Number of metal atoms bonded to an adsorbate

$P_{1}(1) \quad$ Probability of desorption from the ith environment

$P_{i j}(2)$. Probability of diffusion from site $i$ to site $j$

$Q(x) \quad B o n d$ energy ( $\mathrm{kcal} / \mathrm{mol}$ )

Q E Equilibrium bond energy ( $\mathrm{kcal} / \mathrm{mol}$ )

QoA Heat of adsorption of $A$ in the on-top position ( $\mathrm{kcal} / \mathrm{mol}$ )

$Q_{i}, Q_{j} \quad$ Heats of adsorption in sites $i$ and $j$ ( $k \mathrm{cal} / \mathrm{mol}$ )

$Q_{A, n^{*}} \quad$ Heat of adsorption due to $\mathrm{M}-\mathrm{A}$ interactions (kcal $/ \mathrm{mol}$ ) 


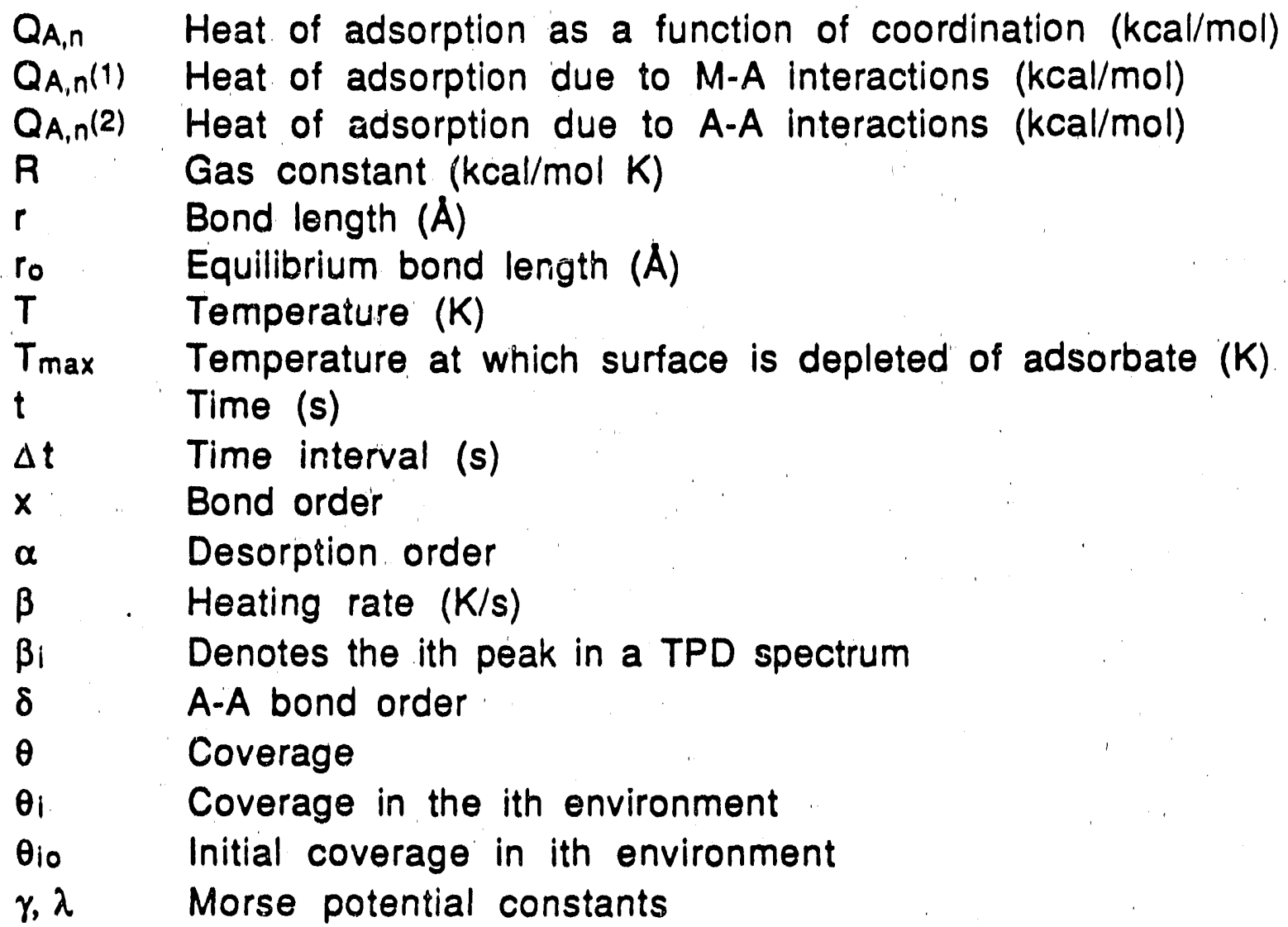




\section{REFERENCES}

[1] J. Falconer and J.A. Schwarz, Catal. Rev.-jci. Eng. 25 (1983) 141.

[2] L.D. Schmidt, Catal. Rev.-Sci. Eng. 9 (1974) 115.

[3] R.J. Cvetanovid and Y. Amenomiya, Catal. Rev. (1972) 21.

[4] G.A. Somorjai, Chemistry in Two Dimensions: Surfaces (Cornell University Press, Ithaca, NY, 1981).

[5] R.H. Fowler and E.A. Guggenheim, Statistical Thermodynamics (Cambridge University Press, 1960).

[6] D.L. Adams, Surface Sci. 42 (1974) 12.

[7] C.G. Goymour and D.A. King, J. Chem. Soc. Faraday I 69 (1973) 749.

[8] D.A. King and M.G. Wells, Proc. R. Soc. London A. 339 (1974) 245.

[9] J.B. Benziger and G.R. Schoofs, J. Phys. Chem. 88 (1984) 4439.

[10] E.S. Hood, B.H. Toby, and W.H. Weinberg, Phys. Rev. Lett. 50 (1985) 2437.

[11] S. Glasstone, K. Laidler and H. Eyring, The Theory of Rate Processes (McGraw-Hill) 1941.

[12] R.C. Baetzold, G.A. Somorjai, J. Catalysis 45 (1976) 94.

[13] O.V. Krylov, M.U. Kisluik, B.R. Shub, A.A. Gezalov, N.D. Maksimova, Y.N. Rufov, Kinetika i Kataliz 13 (1972) 598.

[14] a) V.P. Zhdanov, Surface Sci. 111 (1981) L662; b) V.P. Zhdanov, Surface Sci. 111 (1981) 63; c) V.P. Zhdanov, J. Pavlicek, Z. Knor, and J. Heyrovsky, Catal. Rev. - Sci. Eng. 30 (1988) 501.

[15] H. Ibach, W. Erley and H. Wagner, Surface Sci. 92 (1980) 29.

[16] H. Pfnür, P. Feulner, H.A. Engelhardt, and D. Menzel, Chem. Phys. Letters 59 (1978) 481.

[17] E.G. Seebauer, A.C.F. Kong, and L.D. Schmidt, Surface Sci. 193 (1983) 417.

[18] a) E. Shustorovich, Surface Sci. Reports 6 (1986); b) E. Shustorovich, Acc. Chem. Res. 21 (1988) 183.

[19] Monte Carlo Methods in Statistical Physics, Ed. K. Binder (Springer-Verlag, Berlin) 1979.

[20] IMSL, Subroutine GGUBFS (Houston, TX) 1980.

[21] R.L. Park and H.H. Madden, Jr. Surface Sci. 11 (1968) 188.

[22] a) J.C. Tracy and P.W. Palmberg, Surface Sci. 14 (1969) 274; b) J.C. Tracy and P.W. Palmberg, J. Chem. Phys. 51 (1969) 4852.

[23] R.J. Behm, K. Christmann, G. Ertl, and M.A. Van Hove, J. Chem. Phys. 73 (1980) 2984.

[24] R.J. Behm, K. Christmann, G. Ertl, M.A. Van Hove, P.A. Thiel, and 
W.H. Weinberg, Surface Sci. 88 (1979) L59.

[25] A.M. Bradshaw and F.M. Hoffmann, Surface Sci. 72 (1978) 513.

[26] H.R. Han and L.D. Schmidt, J. of Phys. Chem. 75 (1971) 227.

[27] F. Zaera, E.B. Kollin, and J.L. Gland, Surface Sci. 166 (1986) L149.

[28] J.A. Prybyla, P.J. Estrup, and Y.J. Chabal, J. Vac. Sci. Technol. A 5 (1987) 791.

[29] J.A. Prybyla, P.J. Estrup, S.C. Ying, Y.J. Chabal, and S.B.

Christman, Phys. Rev. Lett. 58 (1987) 1877.

[30] P.J. Estrup, J. Vac. Sci. Technol. 16 (1979) 635.

[31] T.E. Felter, R.A. Barker, and P.J. Estrup, Phys. Rev. Lett 38 (1977) 1138.

[32] S. Semancik and P.J. Estrup, J. Vac. Sci. Technol. 18 (1981) 541.

[33] R.A. Barker, S. Semancik, and P.J. Estrup, Surface Sci. 94 (1980) L162.

[34] P.J. Kuntz and A.C. Roach, J. Chem. Soc. Faraday Trans. 68 (1972) 259.

[35] P.J. Estrup, Department of Chemistry, Brown University, unpublished results.

[36] K. Christmann, R.J. Behm, G. Ertl, M.A. Van Hove, and W.H. Weinberg, J. Chem. Phys. 70 (1979) 4168.

[37] J.N. Russel, Jr., S.M. Gates, and J.T. Yates, Jr., J. Chem. Phys. 85 (1986) 6792.

[38] K. Christmann, O. Schober, G. Ertl, and M. Neumann, J. Chem. Phys. 60 (1974) 4528.

[39] W. Ho, N.J. DiNardo, and E.W. Plummer, J. Vac. Sci. Technol. 17 (1980) 134. 
Chapter IV

\title{
Monte Carlo Simulation of Temperature-Programmed Desorption of Coadsorbed Species
}

\begin{abstract}
A Monte Carlo model is presented for describing the temperature-programmed desorption of coadsorbed species from single-crystal surfaces. Interactions betwsen the adsorbates and the metal surface as well as interactions between the adsorbates are taken into accourit using the bond-order-conservation-Morsepotential (BOC-MP) approach. The number, shape, and location of the peaks is shown to be sensitive to the binding energy, coveiage, and coordination of each coadsorbed species. The presence of a strongly bound coadsorbate on a bcc(100) surface is shown to shift the desorption spectrum for associative desorption of adsorbed atoms to lower temperatures. TFD spectra for the concurrent associative desorption of $A$ atoms and the desorption of $B$ molecules from an $f c c(100)$ surface are of two types: in one case, both species exhibic new low-temperature features far removed from their pure component spectra; in the second case, only the species undergoing associative desurption displays new spectral features. The simulated TPD spectra are in qualitative agreement with experimental results for $\mathrm{H}_{2}$ coadsorbed with strongly bound atomic species on $M O(100)$ and $F e(100)$ surfaces as well as for $\mathrm{CO}$ and $\mathrm{H}_{2}$ coadsorbed on $\mathrm{Ni}(100)$ and $\mathrm{Rh}(100)$ surfaces.
\end{abstract}




\subsection{INTRODUCTION}

The interactions of single adsorbates with metal surfaces have been examined extensively using temperature-programmed desorption (TPD) spectroscopy [1-6]. More recently, TPD spectroscopy has also been used to characterize the behavior of coadsorbed species [7-27]. Such studies can be classified into two typus: those studies in which one spevies is strongly bound to the metal surface and does not desorb during a TPD experiment and those studies in which both species are of comparable binding ensrgies and hence desorb in the same temperature range. The presence of a coadsorbed species can alter significantly the TPD spectrum of the adsorbed species. Strongly bound species tend to shift the TPD peaks for the desorbing species to lower temperature and may even cause individual peaks to merge. Coadsorbed species with comparable heats of adsorption may also produce downscale shifts in the position of TPD peaks and, in addition, give rise to new lowtemperature features.

Theoretical descriptions of TPD spectra for coadsorbed species have been reported, using both continuum and stochastic models. In the continuum approach, the local heat of adsorption is usually described by pairwise-additive energetics. The interactions between adsorbates are assigned either an attractive or repulsive contribution to the local binding energy of the adsorbate, and the magnitude of the adsorbate-adsorbate interaction is treated as an adjustable parameter. The local occupancy of adsorption sites in the continuum models is specified in terms of a distribution function which depends on the average coverage and on the nature of the 
interactions between adsorbates. It should be noted, though, that no a priori basis exists for assigning the relative magnitude and sign of an interaction or for assuming linearly-additive contributions from nearest-neighbor adsorbates. Stochastic models may also use pairwise-additive interactions to describe local energetics but make no assumptions about the distribution of local site occupancies.

Benziger and Schoofs [27] and Sundaresan and Kaza [28] have demonstrated using a continuum approach that the nature and strength of adsorbate-adsorbate interactions as well as the surface mobility of adsorbates can influence the number and shape of TPD peaks for coadsorbed species. Gupta and Hirtzel [29] have used a stochastic model to show that attractive interactions lead to sharper peaks, whereas repulsive interactions lead to broader peaks and, in some instances, multiple peaks. It was also found that the appearance or loss of spectral features was sensitive to the nature and magnitude of the energetic interactions.

This paper describes the simulation of TPD spectra for coadsorbed species using a Monte Carlo model similar to that developed recently [30] for the simulation of TPD spectra for single adsorbates. The model accounts for the effects of surface diffusion and surface coordination, and the effects of local coverage on the activation energy for desorption. Both metal-adsorbate (M-A) and adsorbate-adsorbate ( $A-A)$ interactions are described using the bondorder-conservation-Morse-potential (BOC-MP) approach [31]. The energetics determined with the BOC-MP method differ from the pairwise-additive energetics in two ways. First, the BOC-MP 
method does not assume linearly-additive nearest-neighbor contributions to the total energetics. Rather, the functional form of the contributions of the $M-A$ and $A-A$ interactions to the total energetics are derived relationships within the model framework. Second, the activation energy for associative desorption is a model prediction rather than a model assumption as in the lattice-gas models. In this study, our interest has been the examination of how the coordination, coverage, and strength of bonding of each coadsorbed species influence TPD spectra. In addition, we show that the BOC-MP method can be used to account for the experimentally observed desorption kinetics when two species are coadsorbed on meta! surfaces.

\subsection{THEORY}

Since the basic formulation of the model used for this work has already been discussed [30], we will briefly review the essential features of the model and then demonstrate how it can be extended to include the effects of two species adsorbed on a surface. In the model, each adsorbate is assumed to occupy a fixed type of site and to participate in only two surface processes: desorption and surface diffusion. Since the coverage in the vicinity of each adsorbate may be different, the local heat of adsorption and activation energy for desorption will depend upon the local environment. As described below, the BOC-MP [31] method can be used to determine the energetics associated with each adsorbed species.

The probability of desorption of a given species from site $i$ in the time interval $\Delta t$ can be defined as 
$P_{i}^{(1)}=k_{d, i}^{0} \exp \left(-E_{d, 1} / R T\right) \Delta t$

where $k_{d, l} l^{\circ}$ is the frequency factor for desorption and $E_{d, l}$ is the activation energy for desorption. The value of $\Delta t$ in eq. 1 is chosen so that $P_{i}(1)$ goes to unity at a temperature sufficiently high to guarantee virtually complete desorption from all sites of type $i$.

The rate of desorption, ro, expressed as a turnover frequency based on the number of surface metal atoms, $N_{s}$, is $r_{d}=N_{d} /\left(\alpha N_{s} \Delta t\right)$

where $\mathrm{Nd}_{d}$ is the number of adsorbate atoms or molecules desorbing in the time interval $\Delta t$. The parameter $\alpha$ is 1 for atomic or molecular desorption and 2 for associative desorption. Consequently, $r d$ is the rate of desorption as observed from the gas phase.

Since the activation energy for surface diffusion is $10-15 \%$ of that for desorption, whereas the frequency factors for the two processes are comparable, surface diffusion is expected to be a much more rapid process than desorption. As a consequence, diffusion is not treated as a rate process, but rather, we assume that adsorbate atoms or molecules will reposition themselves nearly instantaneously to achieve an equilibrium state. The equilibrium distribution of adsorbates can be determined from diffusion probabilities where the probability that an adsorbate in site $i$ moves to an adjacent site $j$ is given by [32]:

$$
P_{i j}^{(2)}=\frac{\exp \left[-\left(Q_{i}-Q_{j}\right) / R T\right]}{1+\exp \left[-\left(Q_{i}-Q_{j}\right) / R T\right]}
$$

where $Q_{i}$ and $Q_{j}$ are the heats of adsorption for an adsorbate at sites $i$ and $j$, respectively. Eq. 3 weights diffusion jumps according to the 
magnitude of $\left(Q_{1}-Q_{j}\right)$. It should be noted that if $Q_{1}=Q_{j}$, then $P_{\| j}(2)=$ 0.5 , which indicates that two sites of equal energy have equal probability of occupancy. For a sufficiently large number of jumps, application of eq. 3 yields an equilibrium distribution of adsorbates on a metal surface.

The values of the energetic terms $E_{d, i}$ and $Q_{1}$ appearing in eqs. 1 and 3 are calculated using the BOC-MP approach [31]. With this method, each two-center interaction between an adsorbate atom $A$ and a surface metal atom $M$ is described by a Morse potential $Q(x)=Q_{0}\left(2 x-x^{2}\right)$

where $x$ is the bond order, and $Q_{0}$ is the equilibrium value of the M-A bond energy when $x=1$. The bond order in eq. 4 is defined by $x=\exp \left[-\left(t-r_{0}\right) / a\right]$

where $r$ is the M-A bond length, $r_{0}$ is the equilibrium bond length, and $a$ is a scaling parameter. When $A$ interacts with more than one metal atom, the total heat of adsorption is given by the sum of all two-center interactions. A further assumption of the BOC method is that along a reaction path describing the interactions of a molecular or atomic species with a metal surface, the total bond order is conserved and normalized to unity.

Within the BOC framework, the heat of chemisorption for an isolated atom $A$ on a surface is given by:

$Q_{A, n}=Q_{D A}(2-1 / n)$

where $Q_{O A}$ is the heat of chemisorption of $A$ in the on-top position, and $n$ is the number of metal atoms to which $A$ is coordinated. For an isolated molecular adsorbate $A B$ coordinated via atorn $A$ to $n$ metal atoms, the heat of chemisorption can be approximated by 


$$
Q_{A B, n}=\frac{Q_{Q A}^{2}}{D_{A B}+Q_{D A} / n}
$$

where $D_{A B}$ is the gas phase $A-B$ bond energy.

Eqs. 6 and 7 are valid for isolated adsorbate atoms or molecules on a surface. For higher coverages, however, situations may arise in which more than one adsorbate is bonded to a metal atom and, furthermore, the adsorbates may begin to interact directly with each other. To account for these metal-adsorbate (M-A) and adsorbate-adsorbate (A-A) interactions, the total binding energy of species $A$ is partitioned as follows:

$\dot{Q_{A, n}}=Q_{A, n}^{(1)}+Q_{A, n}^{(2)}$

where $Q_{A, n}(1)$ is the heat of adsorption due to $M-A$ interactions and $Q_{A, n}(2)$ is the heat of adsorption due to $A-A$ interactions. Both $Q_{A, n}(1)$ and $Q_{A, n}(2)$ can be calculated explicitly as a function of the local surface environment, subject to the total bond order of $A$ for both $M$ $A$ and $A-A$ interactions being conserved to unity $[30,31]$.

The value of $Q_{A, n}(2)$ can be expressed as

$$
Q_{A, n}^{(2)}=\sum_{i=1}^{n} \sum_{l=1}^{L} 0.5 D_{A A}\left(2 \delta_{i l}-\delta_{i l}^{2}\right)
$$

where DAA is the A-A bond dissociation energy, and $\delta_{i l}$ is the bond order for the A-A interaction between the $A$ atom coordinated with metal $i$ and the Ith nearest-neighbor $A$ atom also coordinated with metal atom $i$. The summation over $I$ in eq. 9 is to account for all nearest-neighbor 4 atoms. The occurrence of $A-A$ interactions weakens the bond order associated with the $M-A$ interactions and, as 
a consequence, the bond order for each component of an $M_{n}-A$ bond is given by

$x_{i, n}=\frac{1}{n}-\sum_{l=1}^{L} \delta_{i l}$

The value of $Q_{A, n}(1)$ when more than one adsorbate is bonded to an individual metal atom is given by $[30,31]$

$Q_{A, n}^{(1)}=\sum_{i=1}^{n}\left[\frac{Q_{0 A}}{m_{i}}\left(2-\frac{1}{m_{i}}\right)\left(2 x_{i, n}-x_{i, n}^{2}\right)\right]$

where $m_{i}$ is the number of adsorbates bonded to the ith metal atom, and $x i n$ is given by eq. 10 .

Up to this point, we have treated the energetics of a single type of species adsorbed on a surface. We now extend the model to include situations where two types of adsorbates are present on a surface. When coadsorption of species $A$ and $B$ occurs, interactions through the metal between $M, A$, and $B$ are possible as well as direct $A-A, B-B$, and $A-B$ interactions. The total heat of adsorption for species $A$ can again be partitioned (see eq. 8) between the metaladsorbate interactions $Q_{A, n}(1)$ and the direct adsorbate-adsorbate interactions $Q_{A, n}(2)$. A similar expression can be written for the total heat of adsorption of species $B$.

When $A$ and $B$ are coadsorbed on the surface, the calculation of the direct $A-A, B-B$, and $A-B$ interactions can be determined from eq. 9 in the same manner as already described for A-A interactions. The values of DXY and $\delta$ appearing in eq. 9 are then the values for the respective $X-Y$ interactions.

The values of $Q_{A, n}(1)$ and $Q_{B, n}(1)$ can be calculated for the case 
of coadsorption in the following way. The total metal-adsorbate energy, QM-A.B, for the coadsorption of species $A$ and $B$ with a metal atom $M$ is given by [31]:

$Q_{M-A-B}=N_{A} \frac{Q_{A, n}}{n_{A}}\left(2 x_{A}-x_{A}^{2}\right)+N_{B} \frac{Q_{B, n}}{n_{B}}\left(2 x_{B}-x_{B}^{2}\right)$

where $N_{A}$ and $N_{B}$ represent the number of $A$ and $B$ species, respectively, bonded to the metal atom, and $Q_{A, n}$ and $Q_{B, n}$ represent the heats of adsorption for isolated $A$ and $B$ species in their respective $n$-fold coordination (see eqs. 6 and 7). Equation 12 is minimized with respect to $X_{A}$ and $X_{B}$ under the bond order constraint for the metal atom that

$N_{A} \times A+N_{B} \times B=1$

The resulting expressions for $X_{A}$ and $X_{B}$ are:

$x_{A}=\frac{-N_{A} \frac{Q_{B, n}}{n_{B}}+\frac{N_{A} Q_{B, n}}{N_{B} n_{B}}+N_{A} \frac{Q_{A, n}}{n_{A}}}{\frac{N_{A}^{2} Q_{B, n}}{N_{B} n_{B}}+N_{A} \frac{Q_{A, n}}{n_{A}}}$

$x_{B}=\frac{1-N_{A} x_{A}}{N_{B}}$

The magnitude of the total through-metal interactions for $A$ and $B$, $Q_{A, n}(1)$ and $Q_{B, n}(1)$, are then obtained from the expressions:

$Q_{A, n}^{(1)}=\frac{Q_{A, n}}{n_{A}} \sum_{i}\left(2 x_{A i}-x_{A i}^{2}\right) ; Q_{B, n}^{(1)}=\frac{Q_{B, n}}{n_{B}} \sum_{j}\left(2 x_{B j}-x_{B j}^{2}\right)$

The bond orders $X_{A i}$ and $X_{B j}$ are calculated from eqs. 14 and 15, respectively. The summations over $i$ and $j$ in eq. 16 include all of the metal atoms to which an adsorbate is bonded. It should be noted 
that because of the structure of eqs. 14 and 15, the bond orders $x_{A}$ and $X_{B}$ can become negative, depending on the magnitudes of $Q_{A, n}$, $Q_{B}, n, n_{A}, n_{B}, N_{A}$, and $N_{B}$. A negative value of $X_{A}$ or $X_{B}$ simply implies that adsorption under these conditions would not take place.

The expression for $Q_{A, n^{*}}$ derived above (see eq. 8) can be used to determine the activation energy for desorption. For the desorption of a single atom or adsorbed molecule, $A$, the activation energy is given by the heat of adsorption, $E_{d, A}=Q_{A}, n^{*}$. Implicit in this is that adsorption is not an activated process. For homonuclear associative desorption, the activation energy $E_{d, A A^{\circ}}$ is given by [31] $E_{d, A A^{\prime}}=\frac{\dot{Q_{A, n}} \dot{Q}_{A^{\prime}, n}^{*}}{\dot{Q_{A, n}}+\dot{Q}_{A^{\prime}, n}^{*}}$

where $Q_{A, n^{*}}$ and $Q_{A^{*}, n^{*}}$ represent the heats of adsorption of $A$ and $A^{\prime}$, respectively. The two recombining atoms are designated as $A$ and $A^{\prime}$ to denote that the local environments of the two atoms may differ.

The energy and probability formulations described above were incorporated into a Monte Carlo algorithm for simulating the temperature-programmed desorption of adsorbates. The metal surface was represented by a 100 by 100 array of numbered sites, and periodic boundary conditions were used to eliminate edge effects. Adsorbate atoms or molecules were placed on the metal surface lattice to achieve a desired initial coverage, $\theta_{0}$. The temperature was initialized at $T_{0}$ and taken to be constant at this value for the time interval $\Delta t$. A surface site (or pair of adjacent surface sites for associative desorption) was then selected in a random fashion. If the site (or both sites for associative 
desorption) were occupled, the probability of desorption was calculated using eq. 1. The local activation energy for desorption was calculated using eq. 8 (or eq. 17 for associative desorption). The calculated desorption probability was then compared with a random number, $R, 0<R<1$. If $R<P_{1}(1)$, the adsorbate (or pair of adsorbates for associative desorption) was removed from the lattice, and $N_{0}$ in eq. 2 was incremented by one (two for associative desorption). Conversely, if $R \geq P_{1}(1)$, the site (or pair of sites for associative desorption) remained occupied. The preceding steps were repeated $\mathrm{N}_{\mathrm{s}} \theta$ times ( $0.5 \mathrm{~N}_{\mathrm{s}} \theta 2$ times for associative desorption) [30]. After completing the sampling of the surface, the rate of desorption for this time interval was calculated using eq. 2. When coadsorbates existed on the surface, each species was sampled alternately.

A redistribution of the remaining adsorbates was carried out next to account for the effects of surface diffusion. A surface site and an adjacent site were chosen at random. If the surface site were occupied and the nearest-neighbor site were vacant, a probability of diffusion was calculated using eq. 3. Qi was taken as the heat of adsorption in the initial site, and $Q_{\text {f }}$ was taken as the heat of adsorption in the final site. The calculated value of $\mathrm{P}_{\mathrm{ij}}(2)$ was then compared with a random number, $R, 0<R<1$. If $R<P_{i j}(2)$, the adsorbate was moved from the initial site, $i$, to the adjacent site, $j$. Conversely, if $R \geq P_{i j}(2)$, movement of the adsorbate was not allowed. A sufficient number of surface visitations was allowed to attain an equilibrium distribution of adsorbates on the surface. When coadsorbates existed on the surface, each was allowed to 
diffuse alternately.

The desorption and diffusion calculations described above constitute a Monte Carlo Step (MCS). As noted above, the time interval associated with an MCS is $\Delta t$, and over this interval the temperature is constant. At the end of an MCS, the temperature was increased by the increment $\beta \Delta t$, where $\beta$ is the desired heating rate. A new MCS was then carried out at the next temperature. This process was repeated until a temperature was reached for which the surface was depleted of adsorbate. A plot of the desorption rate as a function of temperature for each species then yielded a TPD spectrum for each species. In addition, the average activation energy of desorption could also be determined as a function of the total surface coverage. For both the TPD spectra and the average activation energy profiles, best-fit curves were drawn by eye through the data points. All of the calculations described above were carried out on an IBM 3090 computer. Typical run times were 210 CPU minutes per simulation. Random numbers were generated by the IMSL linear congruential number generator GGUBFS [33].

\subsection{RESULTS AND DISCUSSION}

3.1 Associative desorption in the presence of a fixed coadsorbate The associative desorption of $A$ atoms in the absence of a coadsorbate was considered first. The $A$ atoms were positioned in two-fold bridge sites on a bcc(100) surface at an initial coverage of two and were free to diffuse. Both nearest- and next-nearestneighbor $A$ atoms were considered as possible partners for associative desorption as $A_{2}$. The heat of adsorption for individual $A$ 
atoms was calculated taking into account both $\mathrm{M}-\mathrm{A}$ and $\mathrm{A}-\mathrm{A}$ interactions. The parameter values used for the simulation of the TPD spectra are listed in table 1.

Figure la shows the TPD spectrum for the desorption of $A_{2}$. Three peaks are observed at 285,381 , and $459 \mathrm{~K}$. The dependence of the activation energy on the coverage of $A$, shown in fig. $1 \mathrm{~b}$, exhibits a step-wise decrease as a function of increasing coverage. The flat portions of the activation energy versus coverage curve reflect local surface configurations with relatively constant energetics whereas the steep portions, which occur after changes in coverage due to the evolution of the peaks, correspond to rearrangement and stabilization of the adsorbates by surface diffusion. A map of the surface for $\theta_{A}=1.0$, illustrated in fig. 2 , shows no long range order. Short range order, however, is observed with two $A$ atoms being bonded on the average to every metal atom so as to constitute nearest neighbors. This type of bonding configuration results from the trade-off between the repulsive effects of more than one $A$ atom being bonded to individual metal atoms and the attractive effects of the direct $A-A$ interactions.

Simulations were conducted next for the associative desorption of $A$ in the presence of an immobile coadsorbate $B$. The $B$ atoms were positioned in hollow sites at a coverage of $\theta_{B}=0.25$ in a $p(2 \times 2)$ structure. In calculating the heat of adsorption of $A$ atoms, $M$ $A, M-B$, and $A-A$ interactions were taken into account, but no $A-B$ interactions were included. The binding energy of $B$ was chosen to be sufficiently high so that the rate of $B$ desorption could be neglected. 


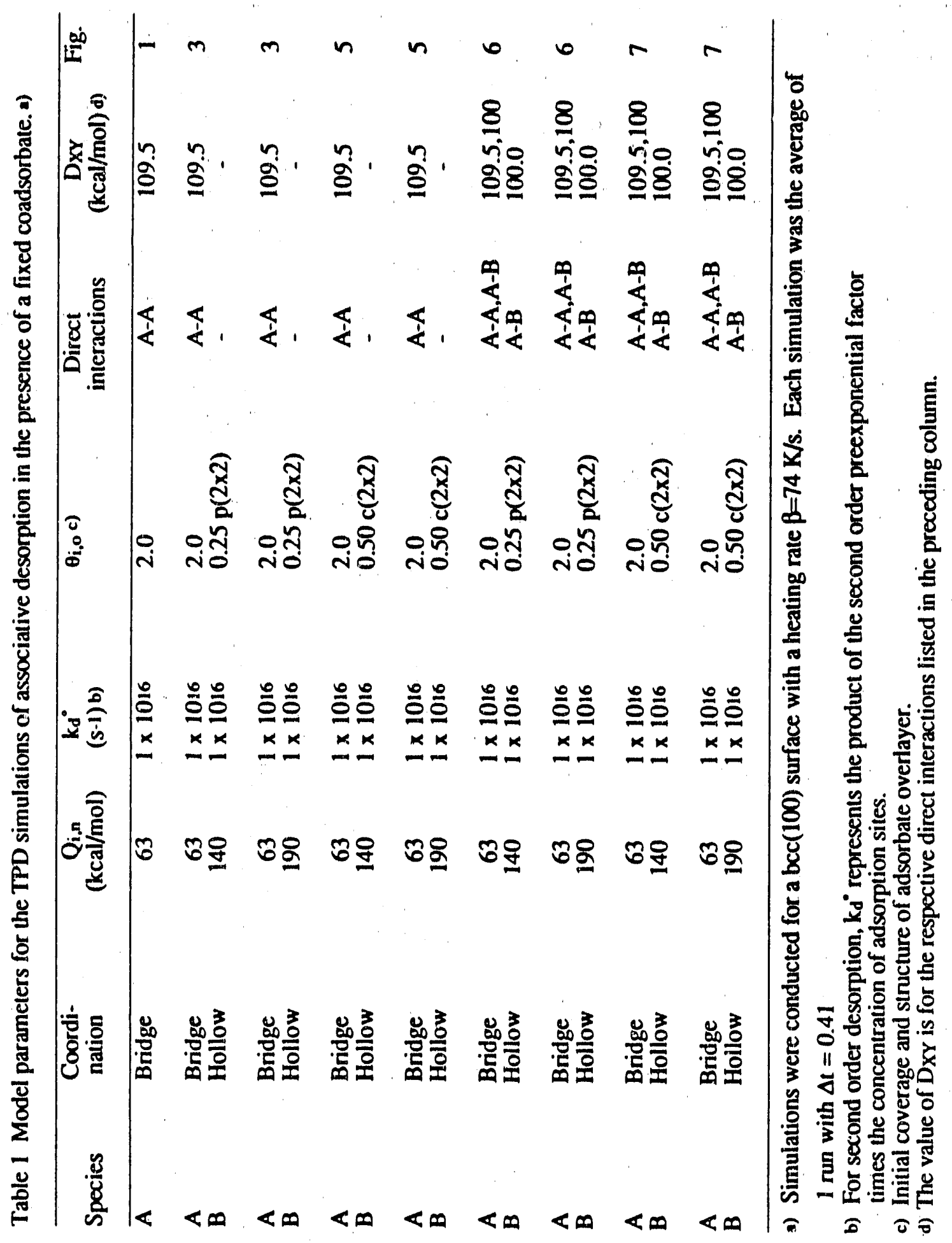


215
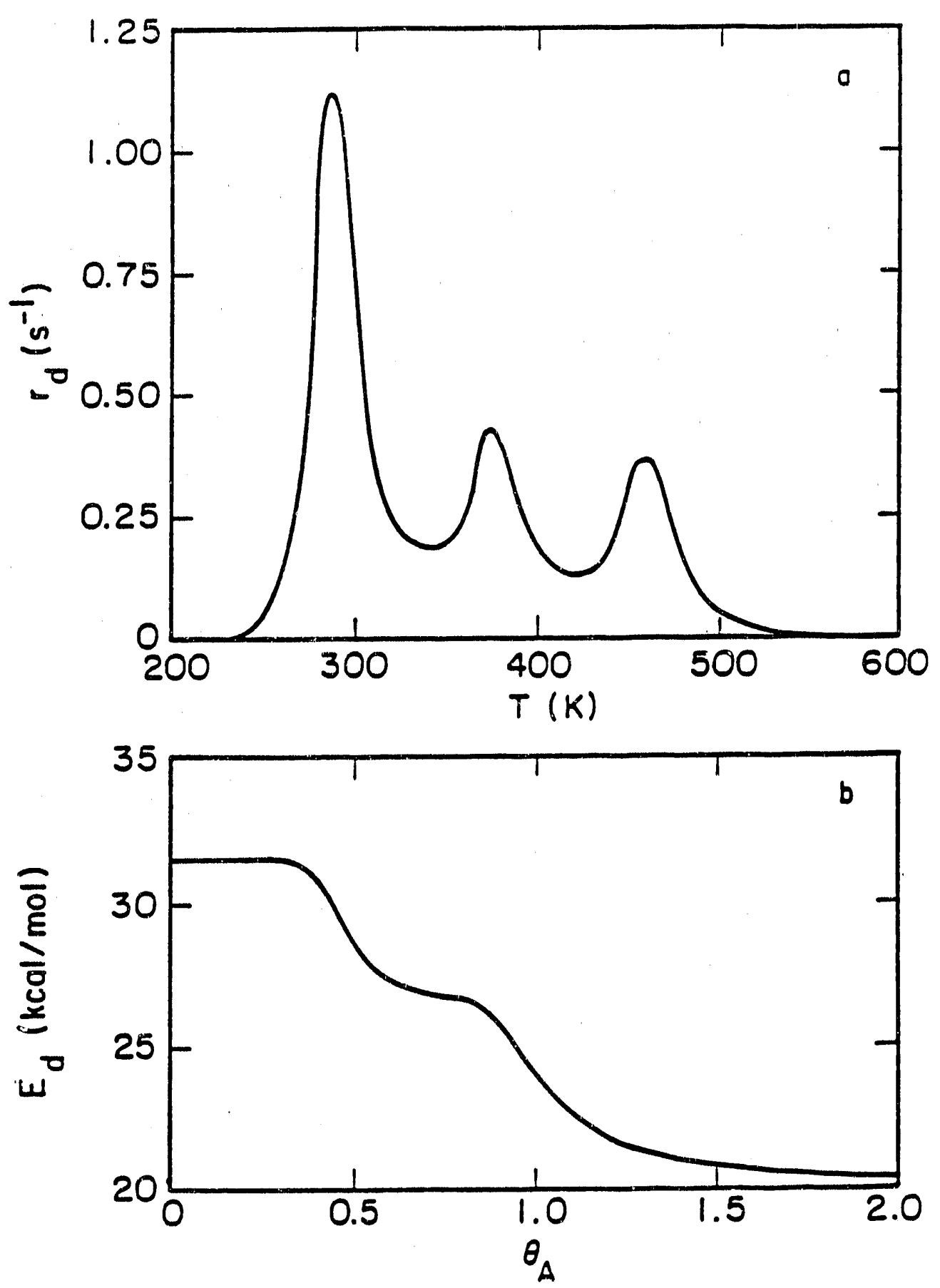

Fig. 1 a. Simulation of $A_{2}$ desorption. See table 1 for model parameters. b. Variation of the average $E_{d}$ with $\theta_{A}$. 
8.0 .00 .0 .80 .80 .80 .0000000000

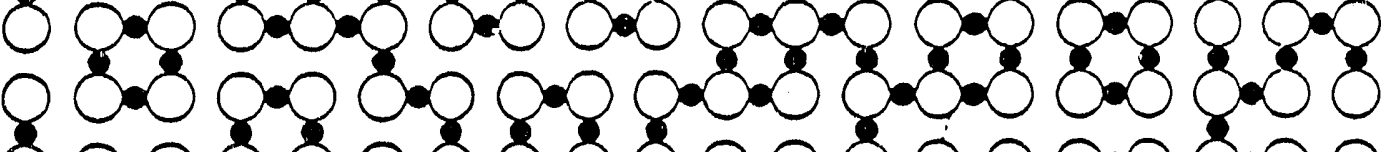

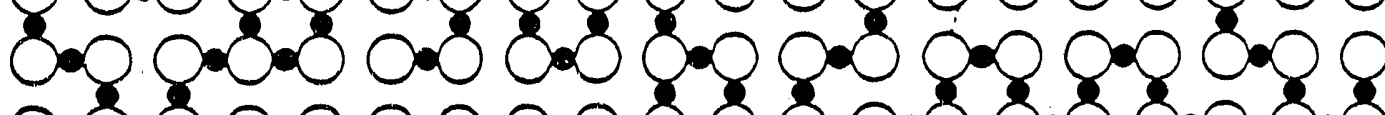

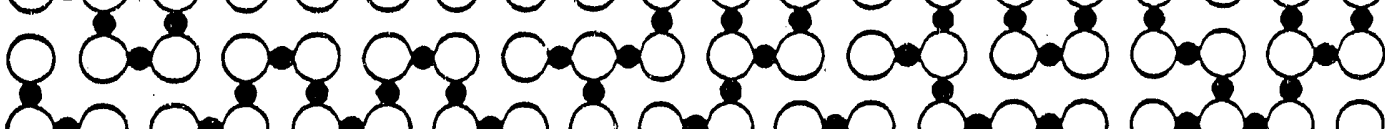

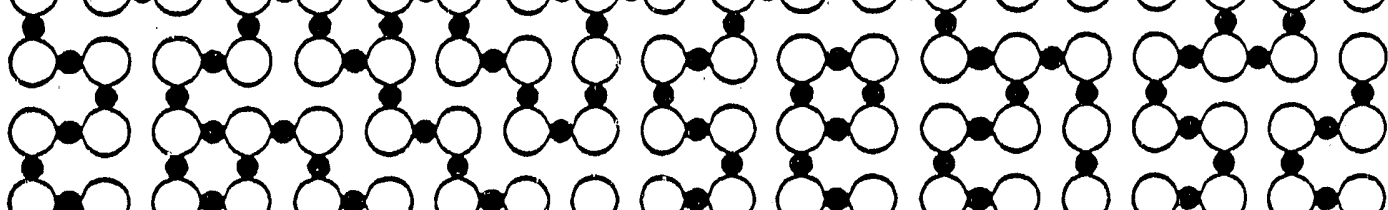
08.80 .808000 .000 .00 .08000

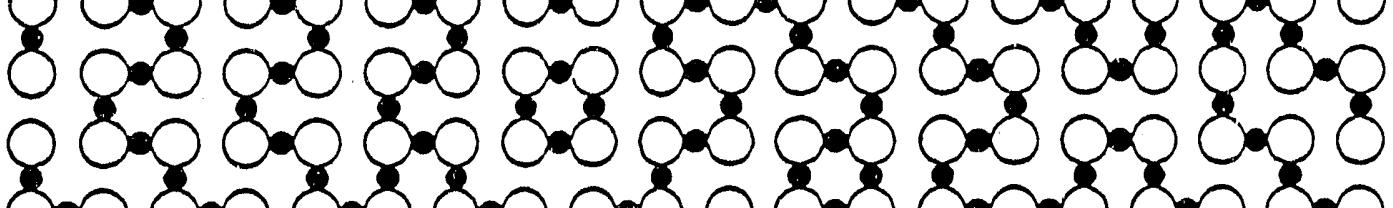

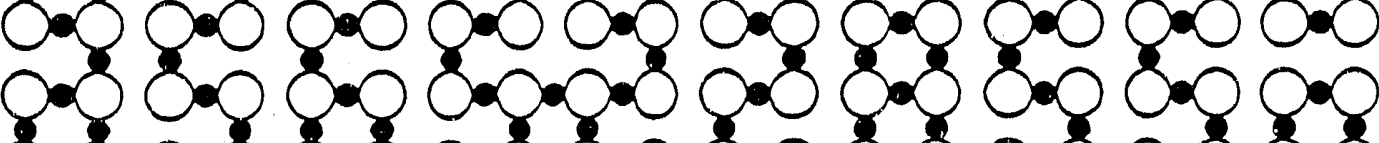
880.08 .08 .08 .0 .00 .08 .08 .088 .0

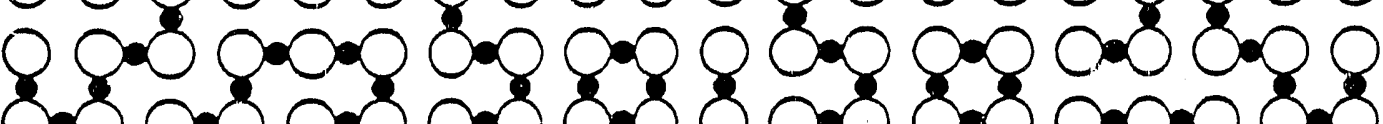

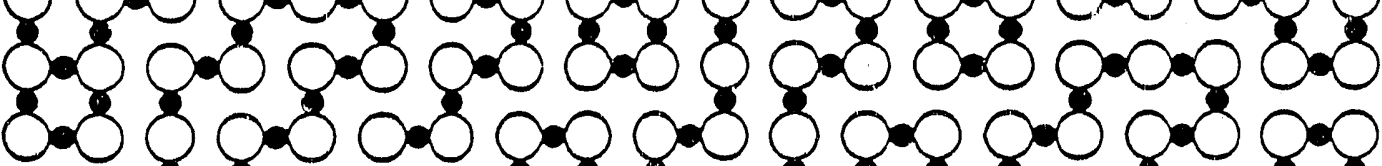

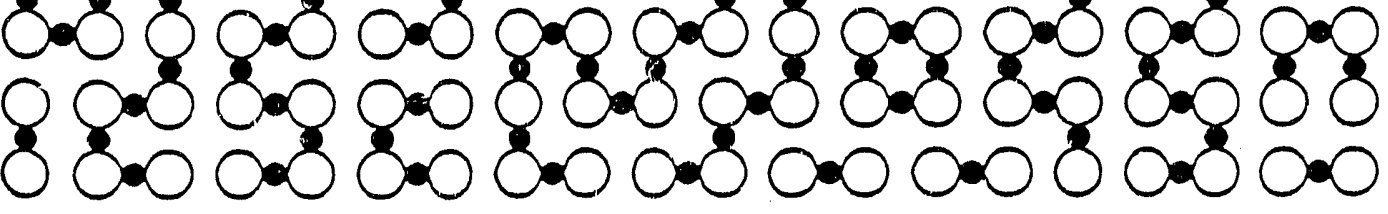

Fig. 2 Surface map for $A(0)$ at $\theta_{A}=1.0$ for the simulation in fig. 1a. The open circles are metal atoms. 
TPD spectra for two different values of the initial binding energy of $B$ are displayed in fig. 3a. For $Q_{B, n}=140 \mathrm{kcal} / \mathrm{mol}$, three peaks for $A_{2}$ are observed. Relative to the spectrum shown in fig. 1a, however, the peaks in fig. $3 a$ are shifted to lower temperatures and exhibit less peak separation. For $Q_{B, n}=190 \mathrm{kcal} / \mathrm{mol}$, three peaks are also observed, and the entire spectrum is shifted to even lower temperatures relative to the spectrum in fig. 1a. The activation energy profiles for both values of $Q_{B, n}$, displayed in fig. $3 b$, show less variation with $\theta_{A}$ than is seen in the case of pure $A$ (see fig. 1b). As $Q_{B, n}$ increases, the activation energy for desorption of $A_{2}$ decreases. This trend is a direct result of the reduction in the heat of adsorption of $A$ due to the decrease in $\times A$. Figure 4 illustrates the surface map for $\theta_{A}=1.0$ when $Q_{B, n}=140 \mathrm{kcal} / \mathrm{mol}$. The local structure seen here is very similar to that in fig. 2 and, in fact, shows little influence due to the presence of the $B$ atoms. This is a direct consequence of the absence of $A-B$ interactions and the equivalence of all the metal atoms (i.e., each metal atom is bonded to one $B$ atom).

Figure 5a shows TPD spectra for two cases in which the coverage of $B$ is increased to 0.5 in a $c(2 \times 2)$ overlayer. For $Q_{B, n}=$ $140 \mathrm{kcal} / \mathrm{mol}$, one peak is seen at $255 \mathrm{~K}$ with a high-temperature shoulder. For $Q_{B, n}=190 \mathrm{kcal} / \mathrm{mol}$, only one peak is observed at 200 K. The corresponding activation energy profiles for $A_{2}$ versus $\theta_{A}$ are given in fig. 5b. Compared to the results for $\theta_{B}=0.25$, the values of the activation energy are lower and exhibit less variation in magnitude with increasing $\theta_{A}$.

A final set of simulations was conducted to illustrate the 
218
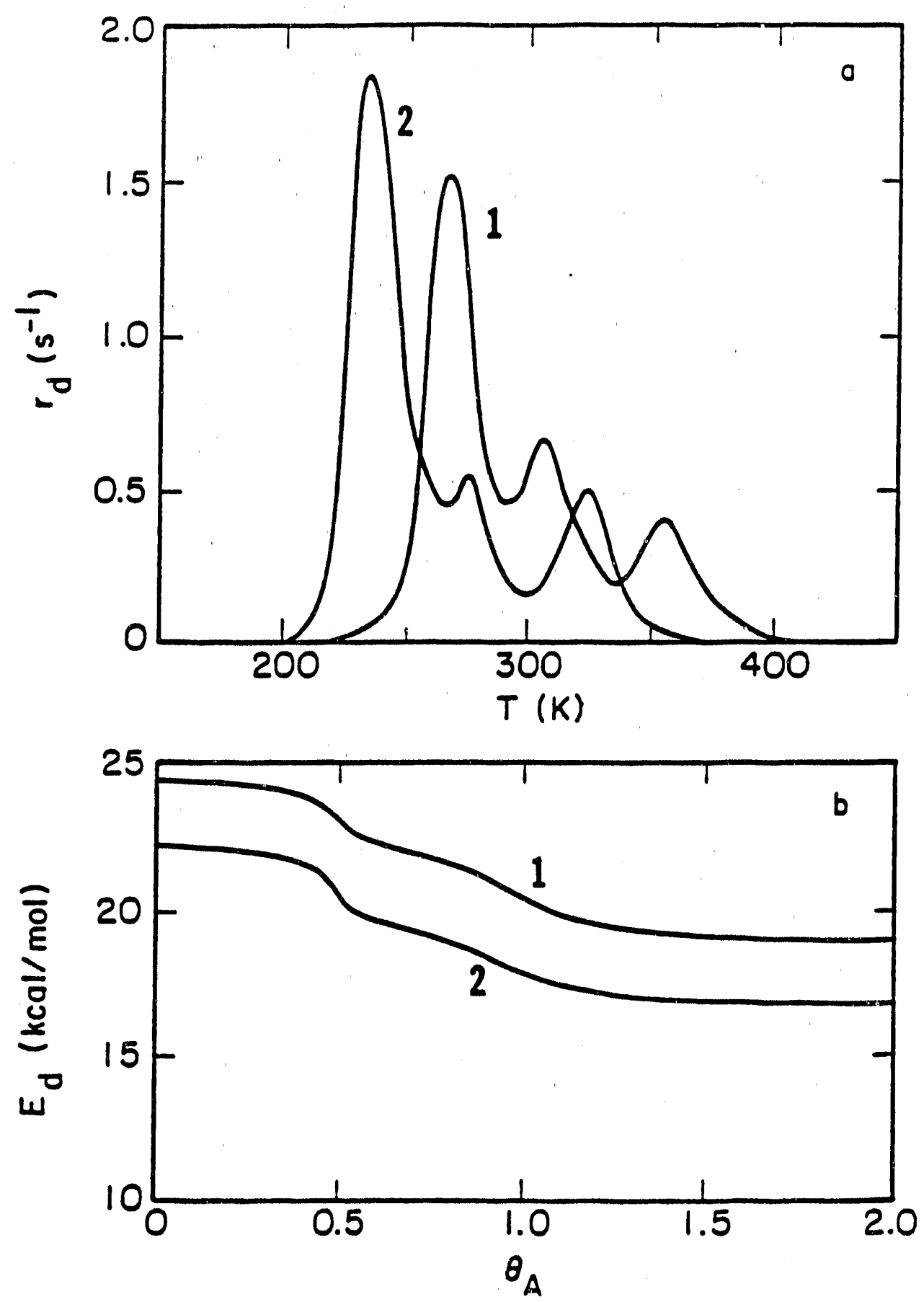

Fig. 3 a. Simulation of $A_{2}$ desorption with $\theta_{B}=0.25$. Curve 1 is for $Q_{B, n}=140 \mathrm{kcal} / \mathrm{mol}$ and curve 2 is for $Q_{B, n}=190$ $\mathrm{kcal} / \mathrm{mol}$. See table 1 for model parameters. b. Variation of the average $E_{d}$ with $\theta_{A}$. 


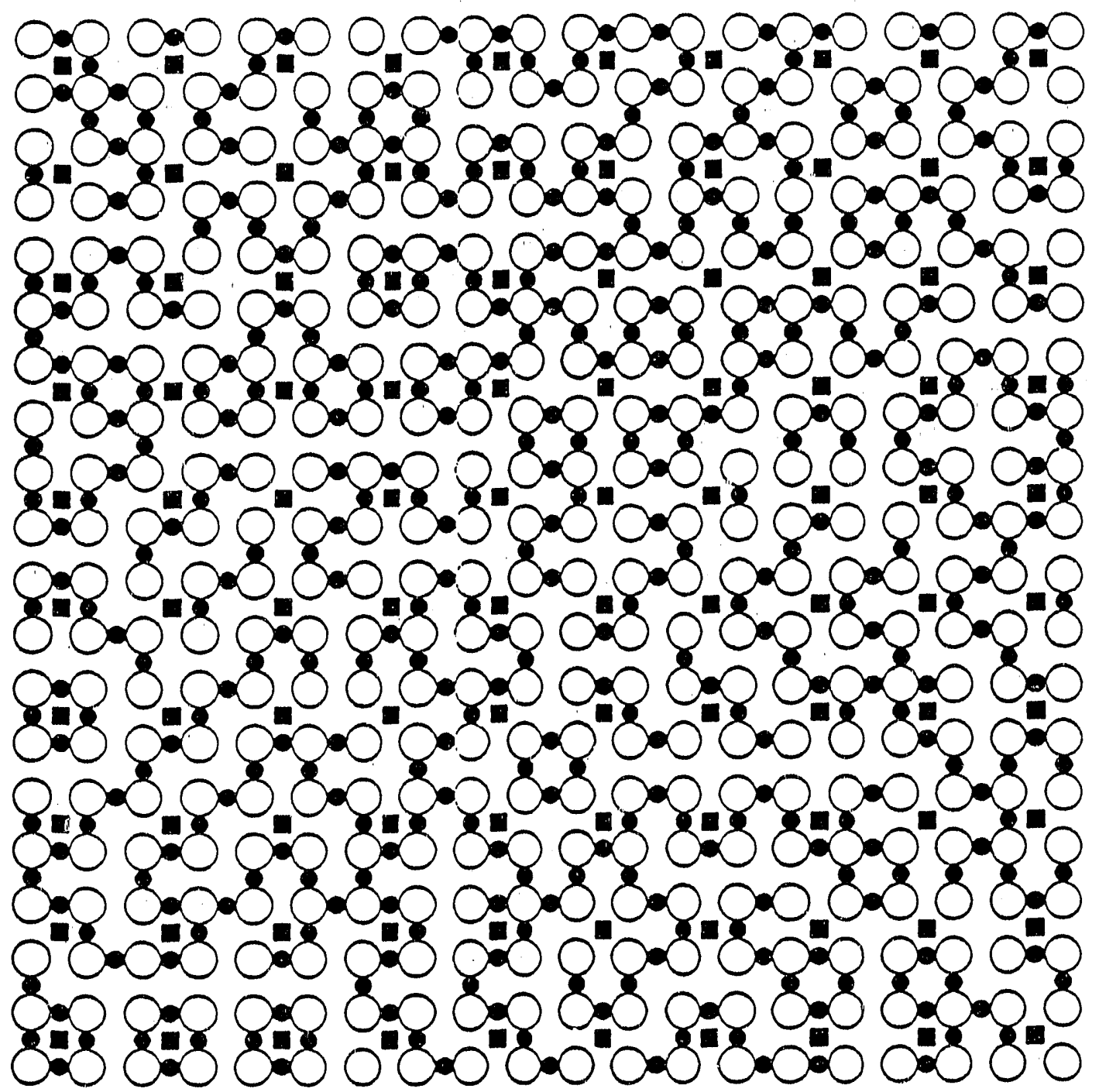

Fig. 4 Surface map for $A(0)$ and $B(\square)$ at $\theta_{A}=1.0$ and $\theta B=0.25$ for the simulations in fig. $3 a$. The open circles are metal atoms. 
220
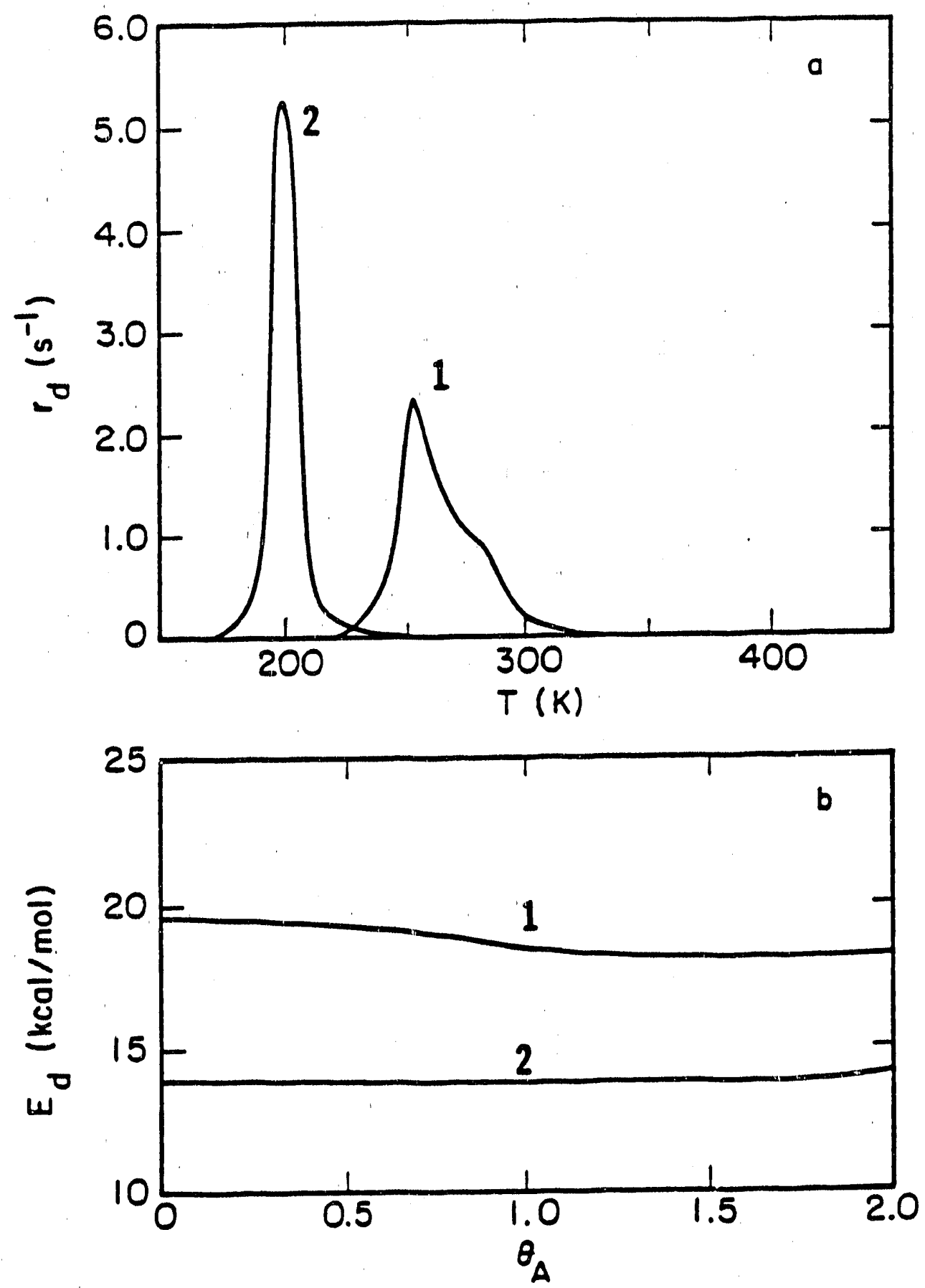

Fig. 5 a. Simulation of $A_{2}$ desorption with $\theta_{B}=0.50$. Curve 1 is for $Q_{B, n}=140 \mathrm{kcal} / \mathrm{mol}$ and curve 2 is for $Q_{B, n}=190$ $\mathrm{kcal} / \mathrm{mol}$. See table 1 for model parameters. b. Variation of the average $E_{d}$ with $\theta_{A}$. 
effects of attractive $A-B$ interactions (see table 1). TPD spectra for $A_{2}$ desorption are shown in figs. $6 a$ and $7 a$. For $\theta_{B}=0.25$, the spectra shown in fig. 6a display three well resolved peaks. The peak positions are shifted relative to the peaks seen in fig. $3 a$ for the case in which $A-B$ interactions are neglected. For $\theta_{B}=0.50$, the spectra shown in fig. 7 a display very little difference in shape from those seen in fig. $5 a$ but are shifted to higher temperatures. The activation energy profiles, displayed in figs. $6 \mathrm{~b}$ and $7 \mathrm{~b}$, are similar in shape to the curves in figs. $3 b$ and $5 b$ but exhibit an approximately 2-3 $\mathrm{kcal} / \mathrm{mol}$ increase in energy for a given coverage which is a consequence of the attractive $A-B$ interactions. The surface map for the case of $\theta_{A}=1.0, \theta_{B}=0.25$, and $Q_{B, n}=140 \mathrm{kcal} / \mathrm{mol}$ is shown in fig. 8. Comparison of figs. 4 and 8 demonstrates that the effect of the $A-B$ interactions is to produce a more ordered distribution of $A$ atoms than in the absence of these interactions. As seen in fig. 8 , most of the $A$ atoms form a square array around each $B$ atom.

The variations in the spectral features discussed above are all attributable to changes in the coverage and binding strength of species $B$. Increasing $\theta_{B}$ and increasing $Q_{B, n}$ both weaken the throughmetal bonding of $A$ which in turn lowers the activation energy barrier for the associative desorption of $A_{2}$. This trend is reflected in all of the simulations presented above which show a progressively stronger down-scale shift in spectrum location with increasing coverage and increasing binding energy of species $B$. The presence of $B$ atoms can also cause the loss of specific spectral features; for example, in the case of $\theta_{B}=0.50, Q_{B, n}=190 \mathrm{kcal} / \mathrm{mol}$, the three peaks for $A_{2}$ observed in the absence of $B$ merge into one 

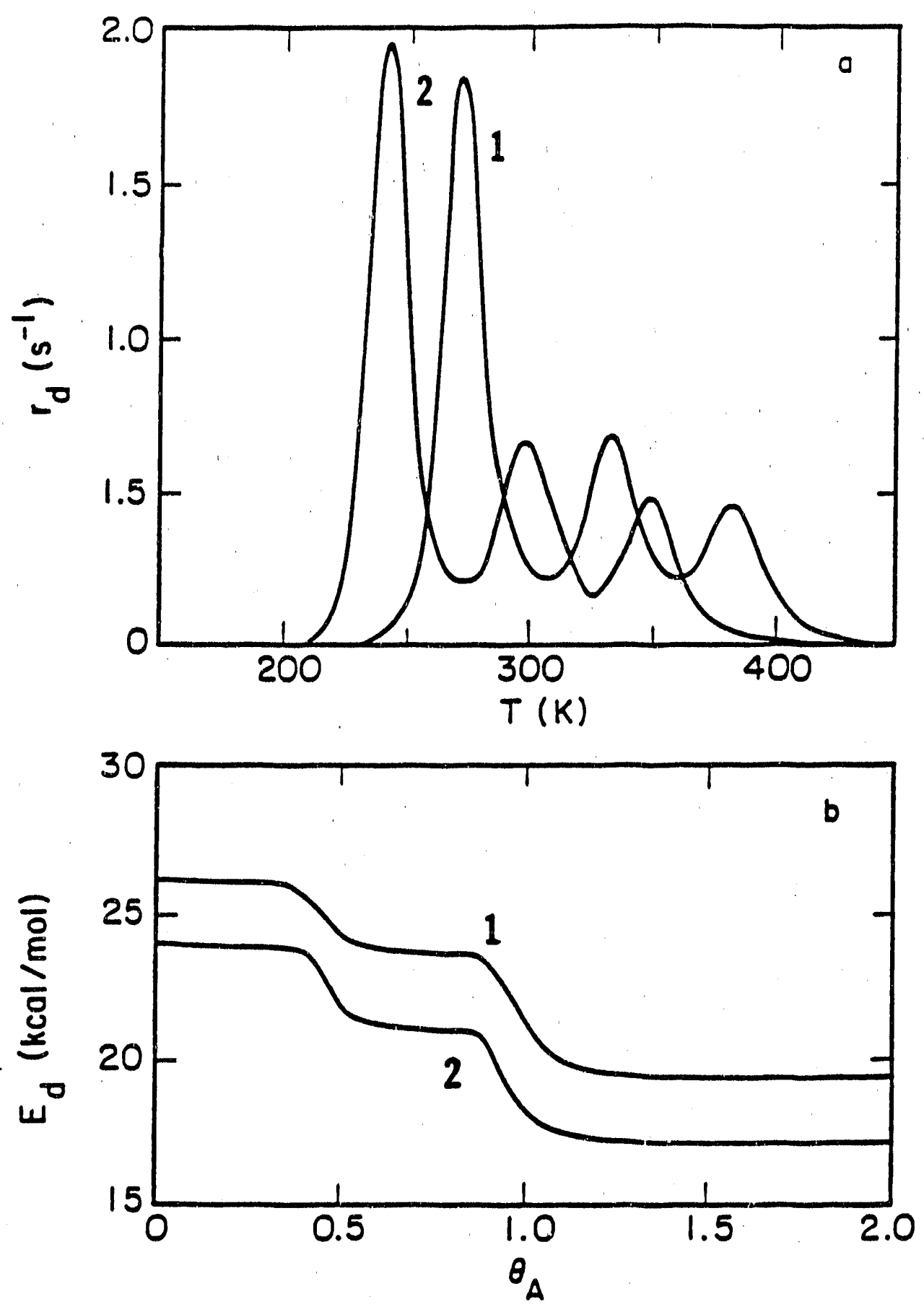

Fig. 6 a. Simulation of $A_{2}$ desorption with $\theta_{B}=0.25$ when attractive $A-B$ interactions occur. Curve 1 is for

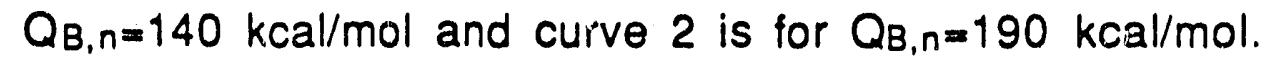
See table 1 for model parameters. b. Variation of the average $E_{d}$ with $\theta_{A}$. 

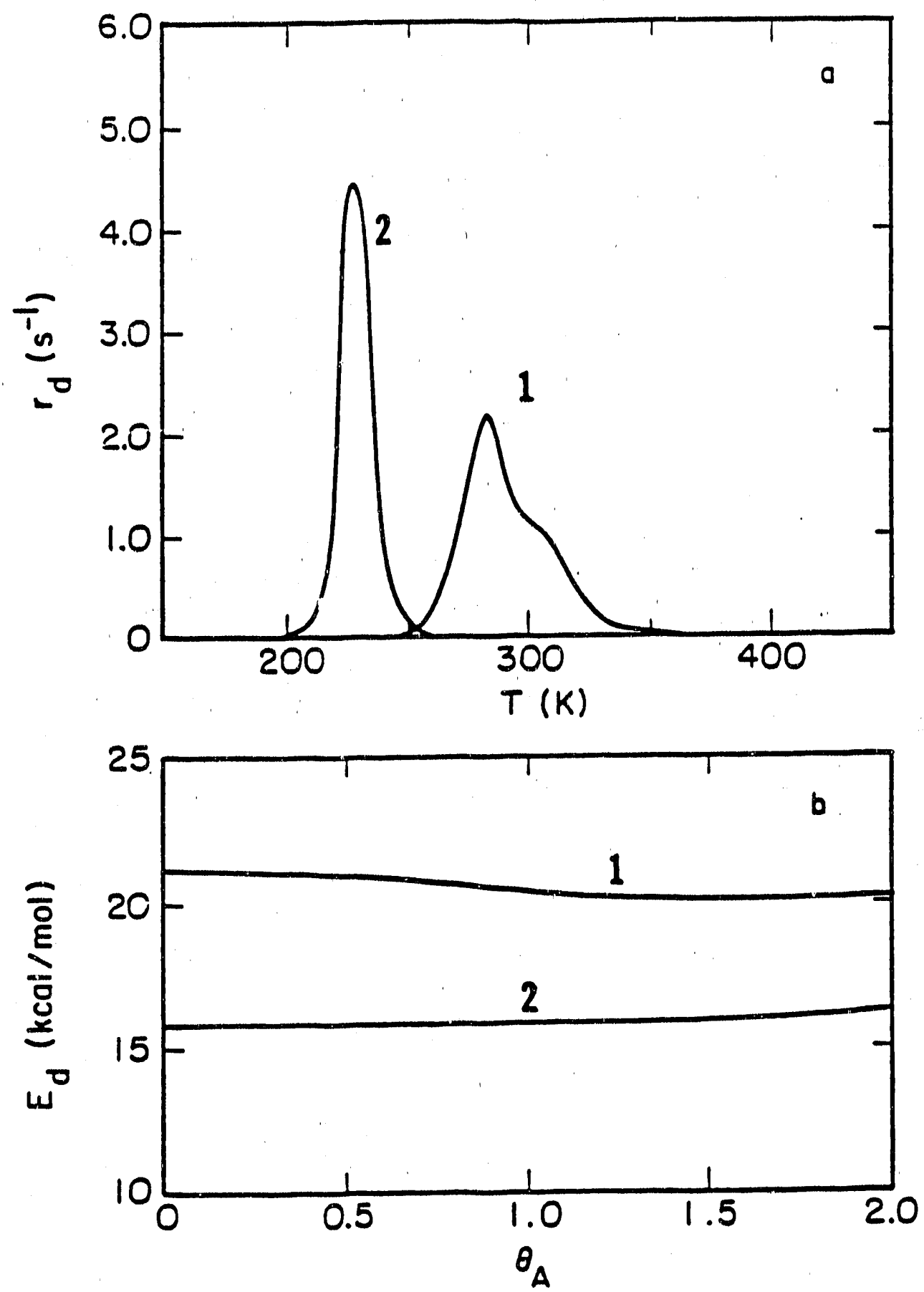

Fig. 7 a. Simulation of $A_{2}$ desorption with $\theta_{B}=0.50$ when attractive $A-B$ interactions occur. Curve 1 is for $Q_{B, n}=140 \mathrm{kcal} / \mathrm{mol}$ and curve 2 is for $Q_{B, n}=190 \mathrm{kcal} / \mathrm{mol}$. See table 1 for model parameters. b. Variation of the average $E_{d}$ with $\theta_{A}$. 


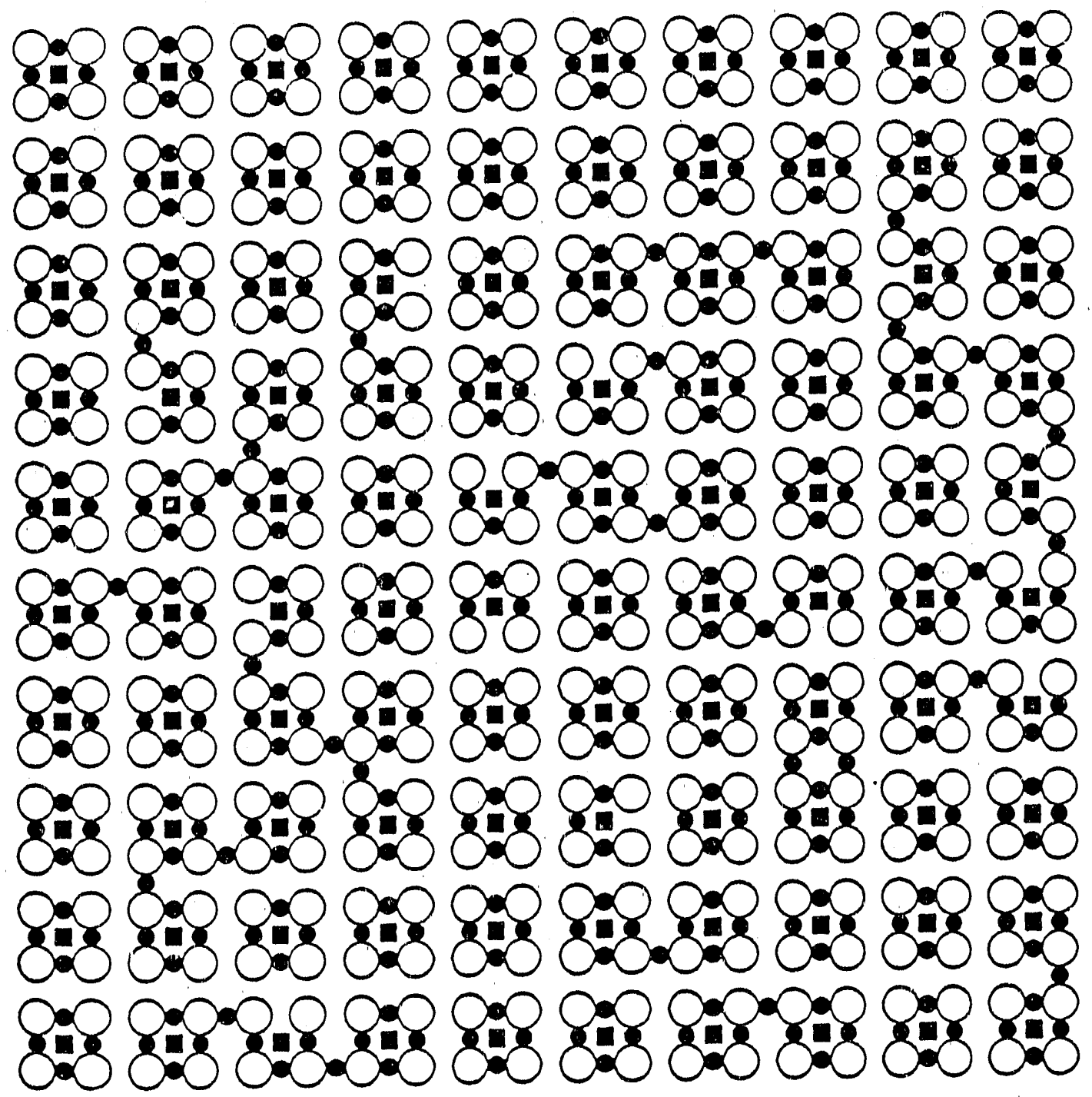

Fig. 8 Surface map for $A(0)$ and $B(\mathbb{D})$ at $\theta_{A}=1.0$ and $\theta_{B}=0.25$ for the simulation in fig. $6 \mathrm{a}$. The open circles are metal atoms. 
peak. When attractive $A-B$ interactions are included, the binding energy increases and with it the activation energy for desorption. This increase, however, is insufficient to completely offset the weakening from the through-metal interactions and a trade-off in spectrum shape and location occurs. Attractive interactions are also seen to strongly affect the structure of the local adsorbate overlayer.

The heat of adsorption of $A$ used in the above simulations is representative for atomic hydrogen adsorbed on early-transition metals, and the range of the heats of adsorption of $B$ used in the simulations span the range of values for atomic nitrogen, carbon, and oxygen adsorbed on early-transition metals. The TPD spectra predicted with these values of the heats of adsorption as inputs to the model are qualitatively consistent with experimental observation For example, hydrogen adsorbed on a clean Mo(100) surface exhibits a TPD spectrum similar to that presented in fig. 1a $[34,35]$. When hydrogen is coadsorbed in the presence of one-half monolayer of nitrogen, the integrated intensity of the hydrogen spectrum is attenuated, and all of the spectral features are shifted slightly to lower temperatures and are more pooply resolved [36]. A second illustration of the effects of coadsorbates is given in ref. [13] which reports on the adsorption of hydrogen on an $\mathrm{Fe}(100)$ surface in the presence of coadsorbed carbon and oxygen. In the absence of a coadsorbate, the maximum hydrogen coverage is 0.50 of a monolayer. The TPD spectrum for this case consists of a single TPD peak similar to the high-temperature peak observed in fig. 1a. Coadsorption of either carbon or oxygen results in an attenuation in 
the hydrogen signal intensity and a shift of the TPD peak to lower temperature.

While the model presented here cannot reproduce the detalls of the experimental spectra, the qualitative features of such spectra are observed. We also note that the structure of eqs. 14 and 15 indicate that for strongly bound coadsorbates, a decrease in the adsorption capacity should occur with increasing strength of adsorption of the coadsorbate. This effect was not observed in the simulations presented here because the coadsorbate coverages and adsorption strength were insufficiently high. Finally, we note that the loss or gain of specific spectral features may not necessarily arise from blocking of a particular binding site, but rather, from the energetic interactions between coadsorbed species.

\subsection{Associative desorption and molecular desorption}

We consider next cases in which two mobile species, labeled for convenience as $A$ and $B$, are coadsorbed on an $f c c(100)$ surface. The energetics are chosen such that both species are capable of desorbing. Atomic species $A$ associatively desorbs to form $A_{2}$, and species B desorbs molecularly. A relevant example would be the coadsorption of atomic hydrogen and molecular carbon monoxide.

We first examine the desorption of pure $A$ in which the $A$ atoms were assigned to hollow sites at an initial coverage of unity, and both nearest- and next-nearest neighbors were considered as desorption partners. The heat of adsorption of an isolated $A$ atom was chosen as $55 \mathrm{kcal} / \mathrm{mol}$. Heats of adsorption as a function of coverage for individual $A$ atoms were calculated taking into account 
both $M-A$ and $A-A$ interactions. Table 2 lists the parameters used. As seen in fig. 9a, the TPD spectrum for $A_{2}$ exhibits a predominant peak at $290 \mathrm{~K}$ and a smaller peak at $355 \mathrm{~K}$ superimposed on a broad, high-temperature tail. The activation energy profile, displayed in fig. $9 \mathrm{~b}$, is constant at $26 \mathrm{kcal} / \mathrm{mol}$ up to a coverage of 0.25 and then decreases steadily until it reaches a value of $20 \mathrm{kcal} / \mathrm{mol}$. A second TPD simulation was conducted for $Q_{A, n}=52 \mathrm{kcal} / \mathrm{mol}$ and is also shown in fig. 9a. It is similar in shape to the previous spectrum except that the high-temperature peak is less pronounced. In addition, the entire spectrum is shifted by $10 \mathrm{~K}$ to lower temperature. The activation energy profile for this case, shown in fig. $9 b$, is similar in shape to the profile when $Q_{A, n}=55 \mathrm{kcal} / \mathrm{mol}$ but is shifted slightly to lower energies as a consec,uence of the initially lower binding energy of the $A$ atoms.

The desorption of molecular species $B$ from the clean surface was considered for two cases of $B$ coordination with the surface, namely, on-top and bridge bonding. The B species were allowed to interact with each other only through the metal atoms. TPD spectra calculated for both on-top and bridge bonding for initial coverages of one-half monolayer in a $c(2 \times 2)$ overlayer are shown in Fig. 9a. Each of these spectra exhibits one peak at $485 \mathrm{~K}$. For bridge-bonded $B$, however, a low-temperature shoulder is also observed. The activation energy profiles, seen in fig. 9b, are initially flat for both species, except that as the coverage approaches 0.35 , the activation energy profile for bridge-bonded $B$ decreases with increasing coverage.

Three cases were considered for the desorption of $A$ and $B$ 


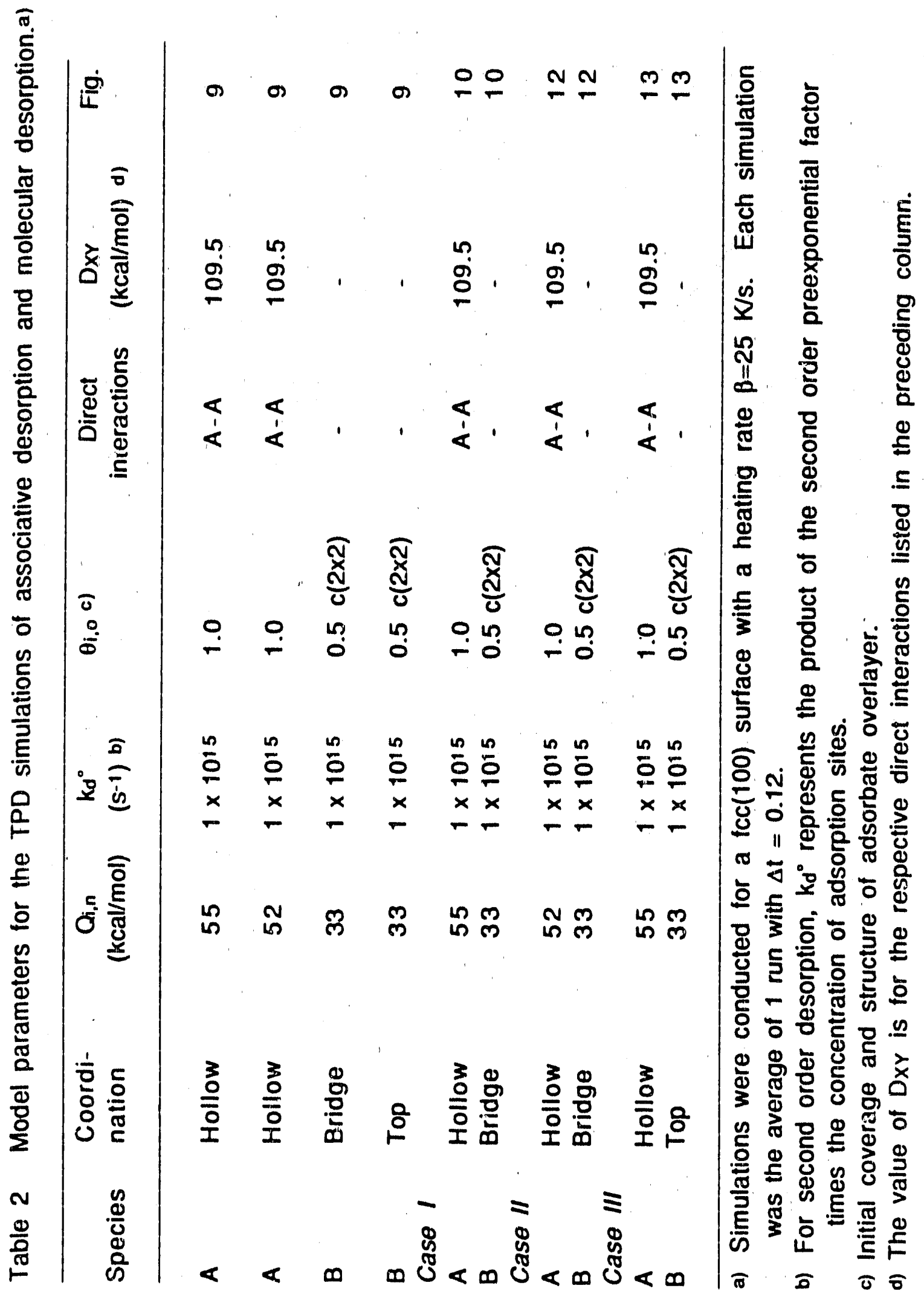




\section{9}
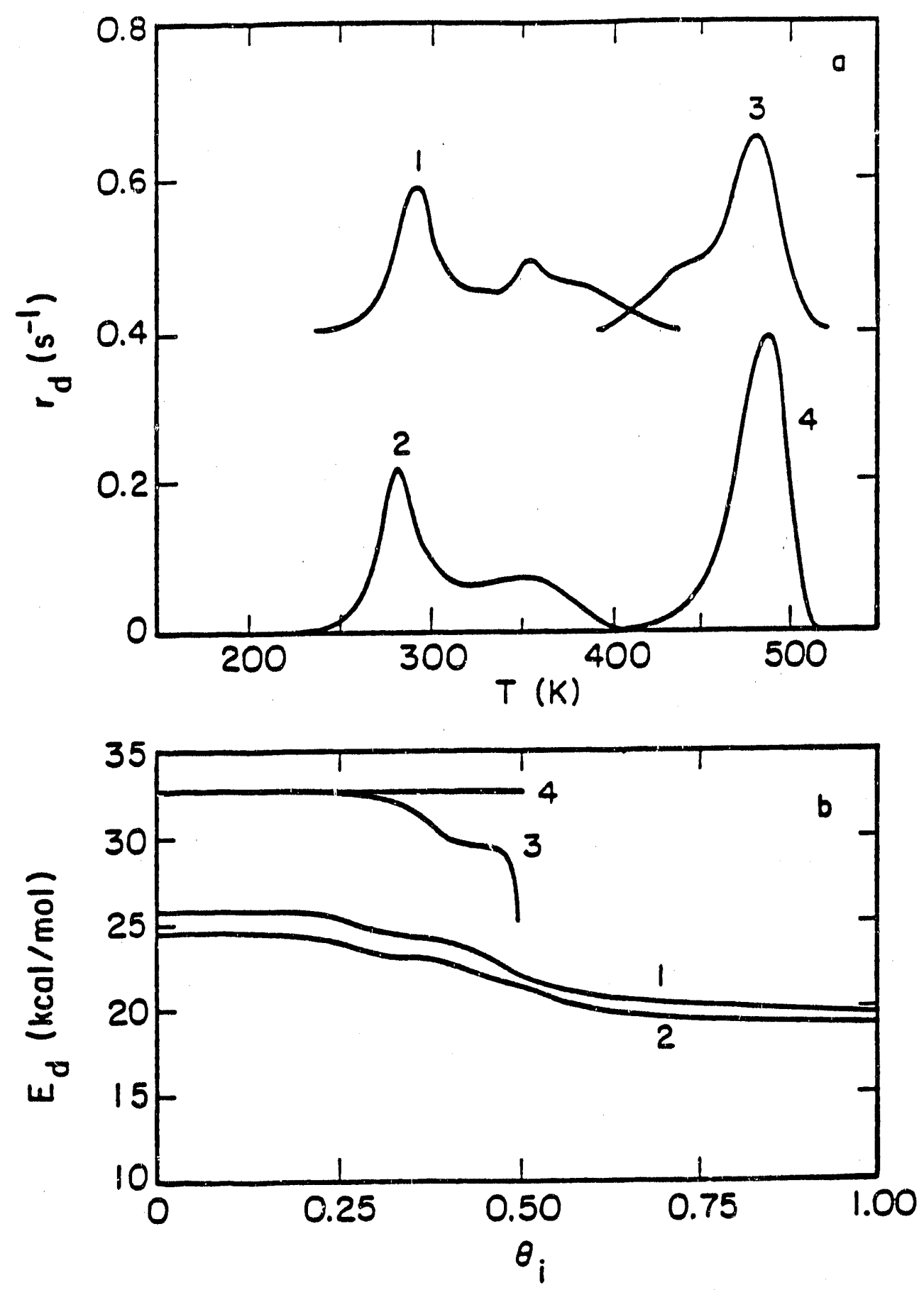

Fig. 9 a. Simulation of pure component desorption for $A_{2}$ and $B$. Curve 1 is for $Q_{A, n}=55 \mathrm{kcal} / \mathrm{mol}$ and curve 2 is for $Q_{A, n=52} \mathrm{kcal} / \mathrm{mol}$. Curve 3 is for bridge-bonded species $B$ with $Q_{B, n}=33 \mathrm{kcal} / \mathrm{mol}$. Curve 4 is for on-top bonded species $B$ with $Q_{B, n}=33 \mathrm{kcal} / \mathrm{mol}$. See table 2 for model parameters. b. Variation of the average $E_{d}$ with coverage. 
when both were present on the surface. In case I, species A occupied hollow positions, and species $B$ occupied bridge positions. The initial coverage of $A$ was unity and that of $B$ was one-half in a $c(2 \times 2)$ overlayer. The binding energies of the isolated species $A$ and $B$ were 55 and $33 \mathrm{kcal} / \mathrm{mol}$, respectively. The binding energy of species $A$ as a function of its local environment was calculated taking into account $A-A, M-A$, and $M-B$ interactions, and the binding energy of species $B$ as a function of its local environment was calculated by taking account $M-B$ and $M-A$ interactions. For both species, no direct $A-B$ interactions were included.

The TPD spectra for case I are shown in fig. 10a. The spectra for both $A$ and $B$ exhibit new features which were not present in the spectra when each species was adsorbed separately (see fig. 9a). The spectrum of species $A$ exhibits a new peak at $240 \mathrm{~K}$ in addition to a high-temperature peak at $300 \mathrm{~K}$ accompanied by a hightemperature tail. The peak at $300 \mathrm{~K}$ and the high-temperature tail occur in the same temperature region as the features observed during the desorption of pure $A$ but with different intensities. The spectrum for $B$ exhibits a low-temperature peak at $230 \mathrm{~K}$, a small peak at $355 \mathrm{~K}$, and a more intense peak at $480 \mathrm{~K}$. The peaks at $230 \mathrm{~K}$ and $355 \mathrm{~K}$ are new, while the peak at $480 \mathrm{~K}$ appears in the same region as the spectrum for pure $B$.

The activation energy profiles, shown in fig. 10b, differ significantly from the profiles found in the case of single component desorption (see fig. 9b). The profile for species $A$ is shifted to lower energies by about $1.4 \mathrm{kcal} / \mathrm{mol}$ for most of the coverage range between 0.10 to 1.0. The curve for species $B$ shows very strong 

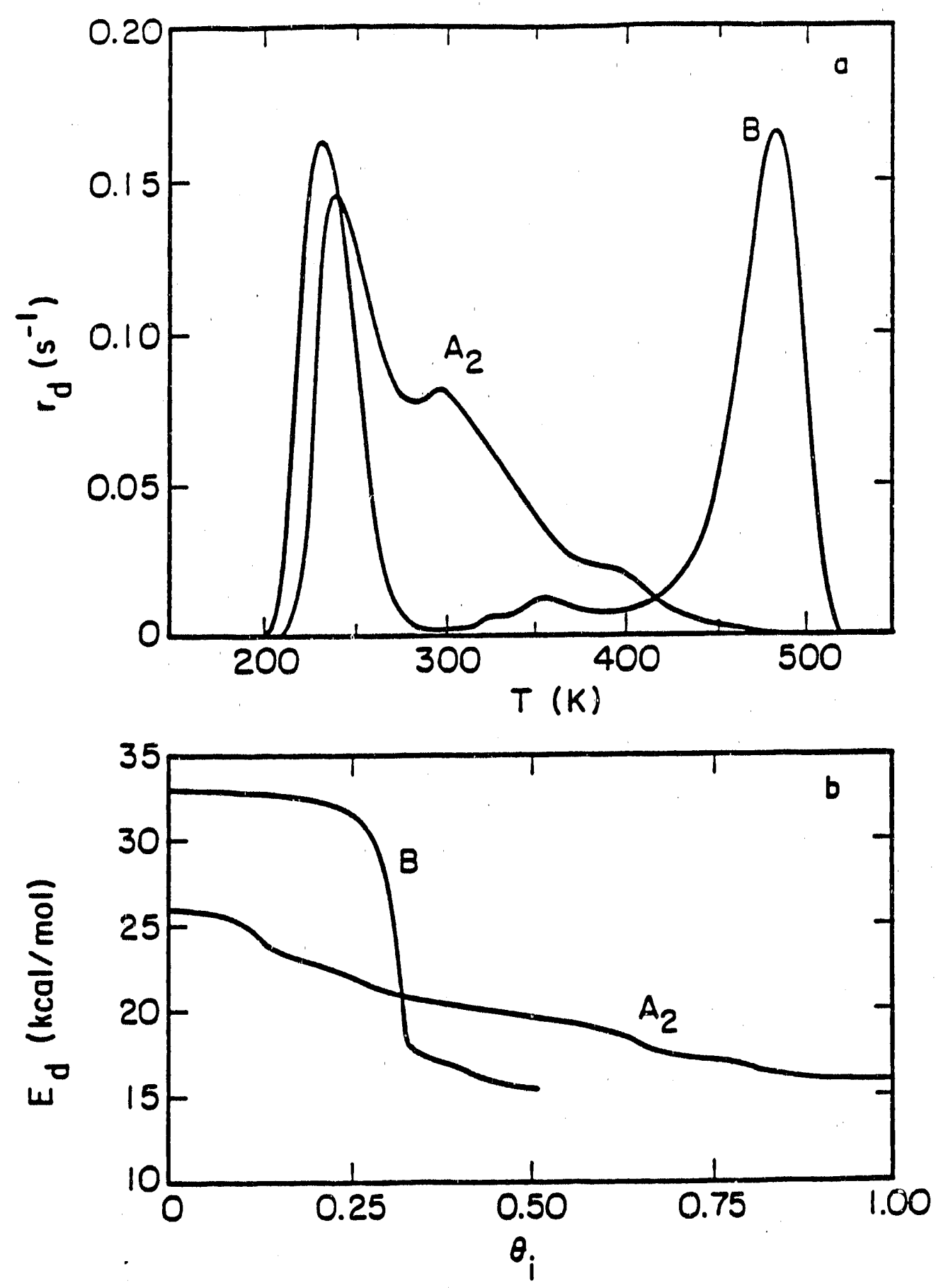

Fig. 10 a. Simulation of $A_{2}$ and $B$ codesorption for case I. See table 2 for model parameters. b. Variation of the average Ed with coverage. 
coverage-dependent behavior, exhibiting a sharp decrease at $\theta_{\mathrm{B}}=$ 0.30. It is significant to note that the activation energy for the desorption of $B$ at the start of the TPD (i.e. at $\theta_{B}=0.50$ ) is lower than the corresponding value for the desorption of $A_{2}\left(\theta_{A}=1.0\right)$. A surface map for $\theta_{A}=0.51$ and $\theta_{B}=0.32$, shown in fig. 11, displays segregation of the the two species into regions rich in $A$ and $B$. Furthermore, species $B$, which was initially ordered in a $c(2 \times 2)$ overlayer, is now seen to be densely packed in the B-rich regions.

In case II, the binding energy of an isolated species $A$ was decreased from 55 to $52 \mathrm{kcal} / \mathrm{mol}$. The binding energy and coordination of $B$ remained the same as for case 1 . The calculated TPD spectra are shown in fig. 12a. Species A desorbs predominantly at low temperature with a peak at $230 \mathrm{~K}$ and a broad, hightemperature tail. Species $B$ exhibits peaks at 370 and $480 \mathrm{~K}$, and no peak at $230 \mathrm{~K}$. The activation energy profiles as a function of coverage are shown in fig. $12 \mathrm{~b}$. The curve for $A$, as compared with the curve for $A$ in case $I$ in fig. 10b, is lower for a given coverage and decreases more rapidly to its value at high coverage. The profile for species B, however, is much closer in shape and location to that observed for the desorption of pure B (see fig. 9b). It is interesting to note that reducing the value of $Q_{A, n}$ from 55 to $52 \mathrm{kcal} / \mathrm{mol}$ almost totally eliminates the influence of adsorbed $A$ on the desorption of $B$. The large change in the appearance of the desorption spectra for a relatively small change in the initial binding energy of species $A$ damonstrates the sensitivity of desorption kinetics to the relative binding energies of the coadsorbed species. 


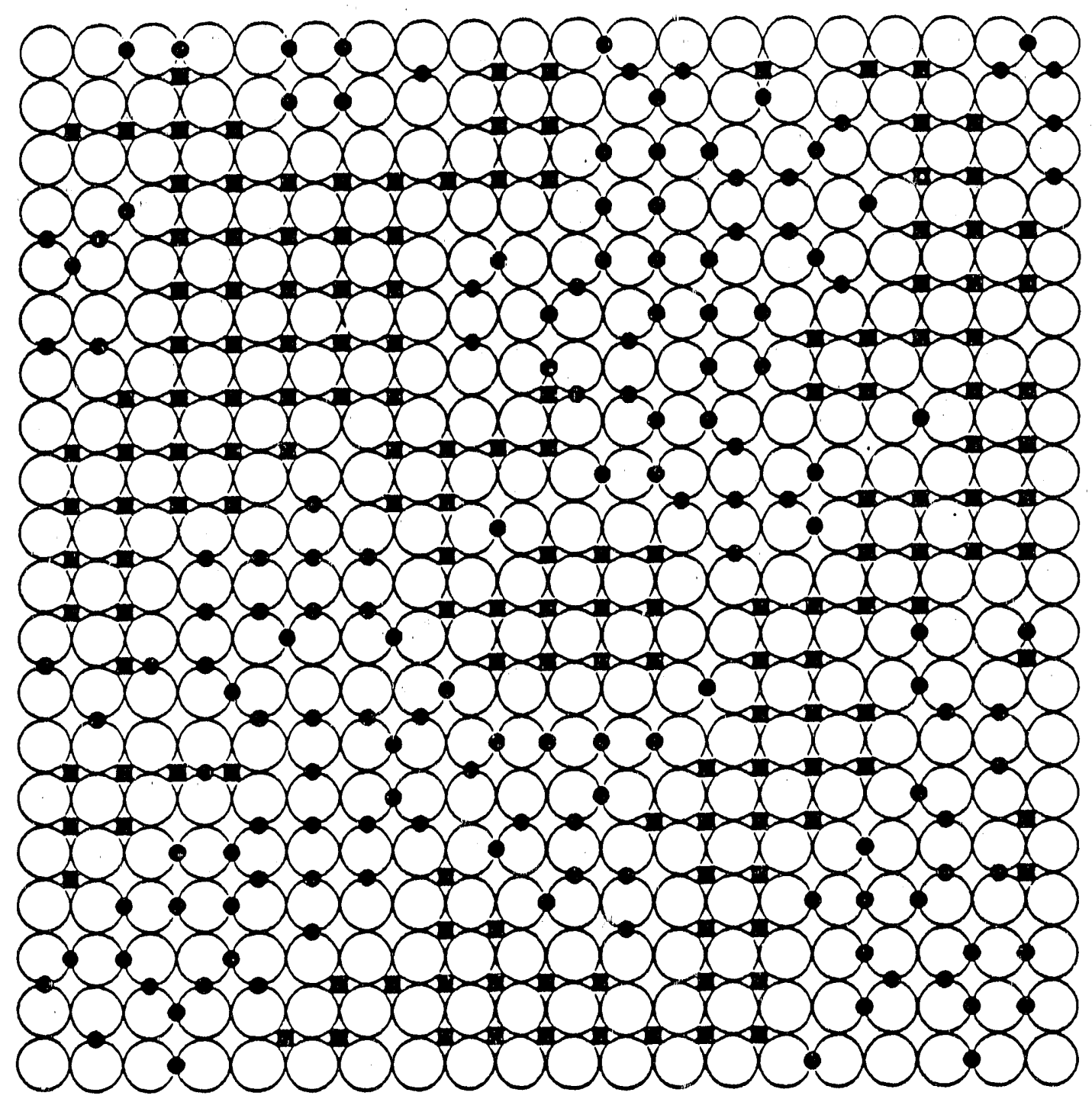

Fig. 11 Surface map for $A(\nabla)$ and $B(0)$ at $\theta_{A}=0.51$ and $\theta B=0.32$ for the simulation in fig. 10a. Open circles are metal atoms. 

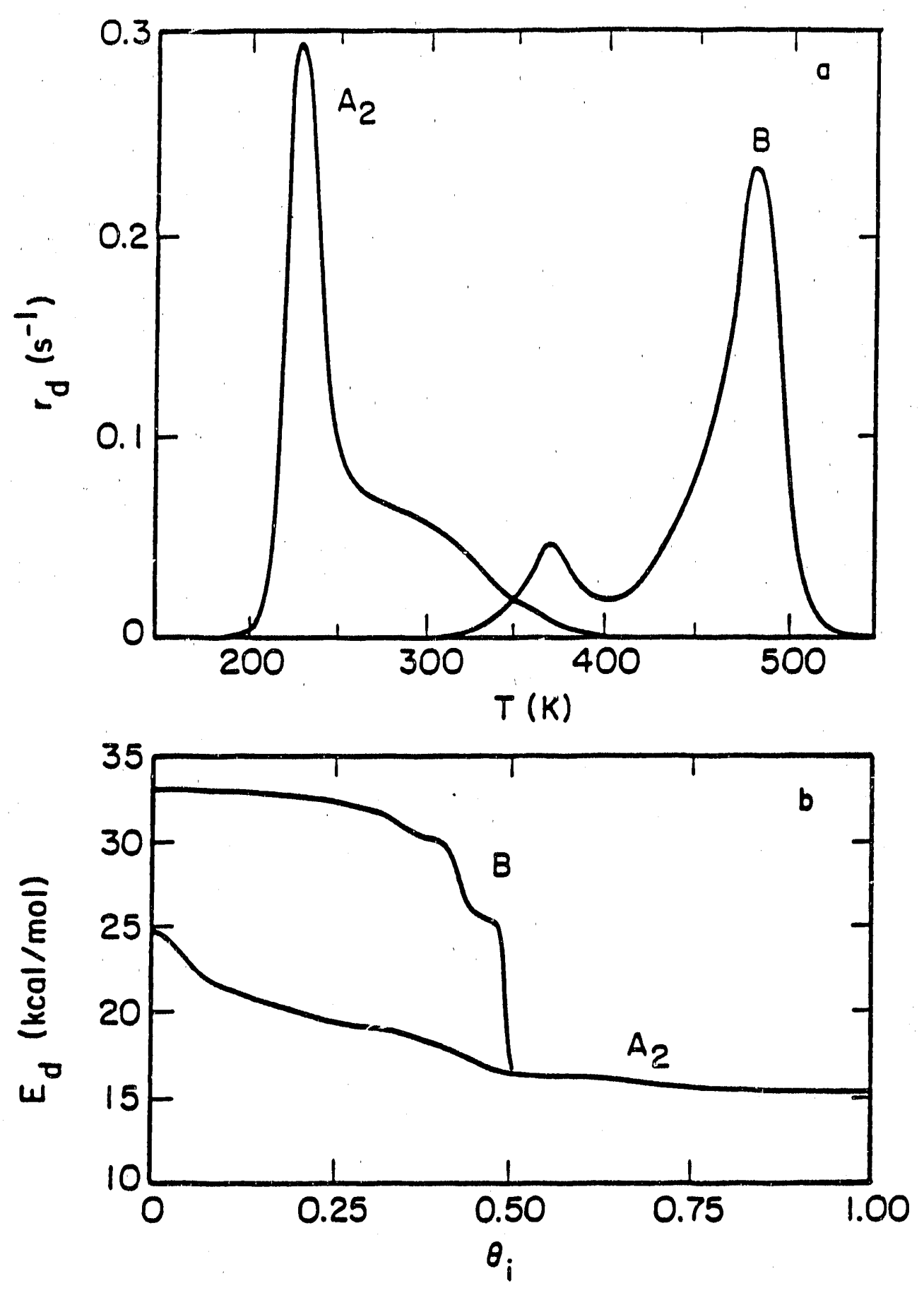

Fig. 12 a. Simulation of $A_{2}$ and $B$ codesorption for case II. See table 2 for model parameters. b. Variation of the average Ed with coverage. 
In case III, the binding energy for species A was the same as that in case 1 (see Table 2). Species B was positioned on on-top sites in a $c(2 \times 2)$ overlayer at an initial coverage of 0.5 . The TPD spectra for this set of conditions are shown in fig. 13a. Species $A$ desorbs with a peak at $225 \mathrm{~K}$ and exhibits a high-temperature tail. Compared to the spectrum for $A_{2}$ desorption in the absence of $B$ (see fig. 9a), the peak of the principal feature for $A_{2}$ is shifted to lower temperatures by $65 \mathrm{~K}$. The spectrum for species $B$ is relatively unchanged from that seen in fig. 9a. The activation energy profiles are shown in fig. 13b. The profile for species $A$ is lower than that calculated for the desorption of pure $A$. The activation energy profile for species $B$, however, is quite similar to that for the desorption of pure $B$. This is not surprising, since the desorption of $B$ occurs only after the surface has been substantially depleted of $A$. The surface map for case $I I I$ is shown in fig. 14. As in fig. 11, segregation of $A$ and $B$ is observed. Since species $B$ can only be affected by species $A$ via through-metal interactions, only those $B$ species located at the periphery of B-rich regions are affected energetically by the presence of $A$.

To summarize, the cases considered above show two general types of behavior: in case 1, the spectra of both adsorbates are strongly influenced by interactions between the coadsorbed species and new features are observed; in cases II and III, associative desorption of the atomic adsorbates is influenced by the molecular species, but the molecular species are only mildly affected by the atomic adsorbates. It is also noted that the appearance or loss of spectral features is determined by the nature and strength of the 

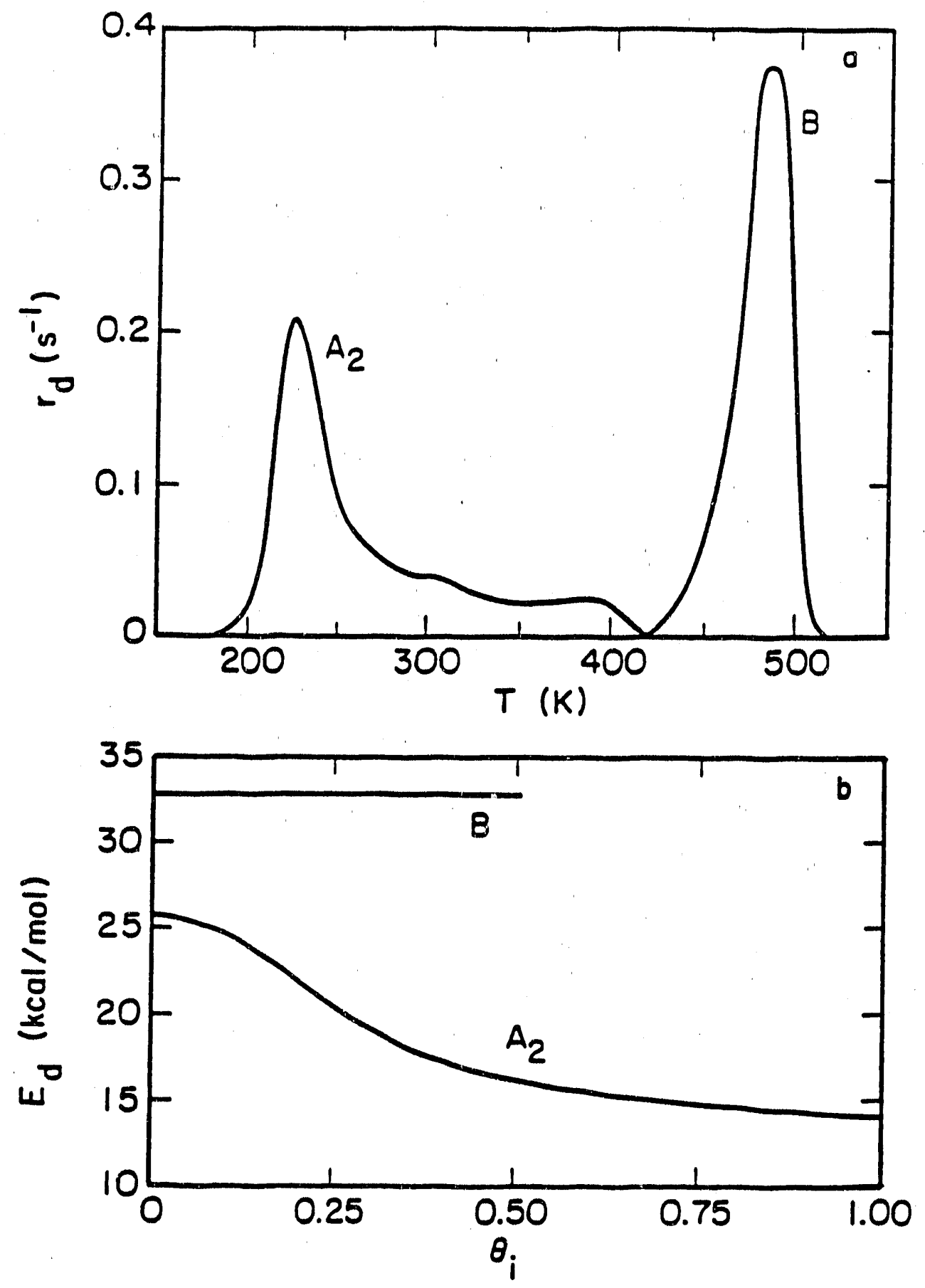

Fig. 13 a. Simulation of $A_{2}$ and $B$ codesorption for case III. See table 2 for model parameters. b. Variation of the average Ed with coverage. 


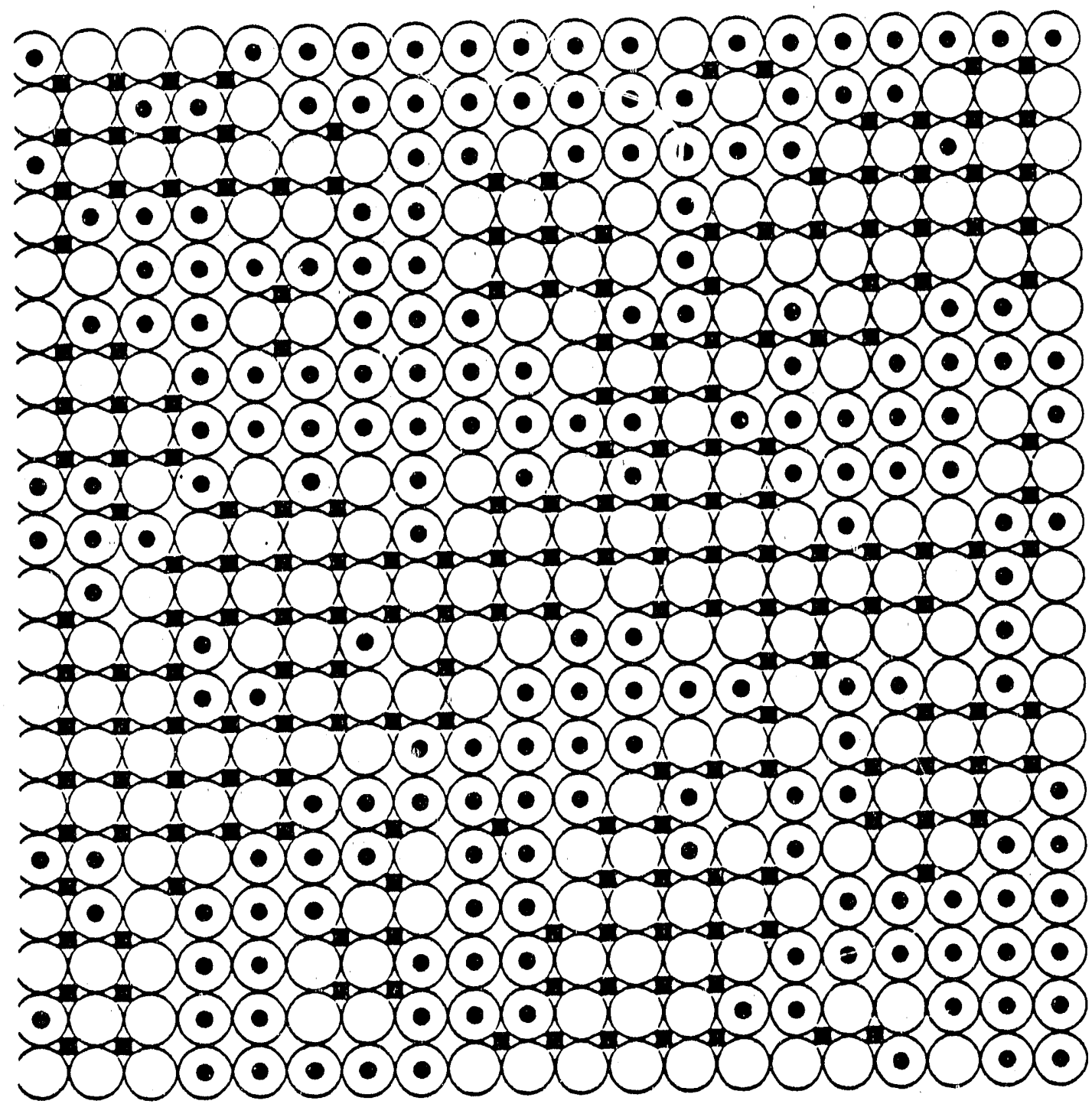

Fig. 14 Surface map for $A(\mathbb{D})$ and $B(0)$ at $\theta_{A}=0.52$ and $\theta_{B}=0.50$ for the simulation in fig. 13a. The open circles are metal atoms. 
interactions between species and not by the loss or creation of new binding states.

The heats of adsorption for $A$ and $B$ used in the above simulations are representative for atomic hydrogen and molecular carbon monoxide, respectively, adsorbed on Group VIII transition metals. The TPD spectra predicted with these values of the heats of adsorption as inputs to the model are qualitatively consistent with experimental observation. For example, the spectra for hydrogen and carbon monoxide adsorbed alone on a $\mathrm{Ni}(100)$ face each exhibit a single peak [16-18], as shown in fig. 15 . Figure 15 also shows that when carbon monoxide and hydrogen are coadsorbed, lowtemperature peaks appear for both species. Comparison of figs. 10a and 15 shows that the spectra simulated for the codesorption of $A$ and $B$ under the conditions of case I resemble qualitatively the spectra observed experimentally. This suggests that the simultaneous evolution of two species at temperatures far below those required to desorb the pure components may simply be a manifestation of the interaction between the coadsorbed species. It is also interesting to note that the segregation of the adsorbates $A$ and $B$ is driven by the attractive interaction between $A$ atoms. When this interaction is strong enough to counter the repulsive effects arising from multiple $A$ atoms being bonded to individual metal atoms, segregation into regions rich in $A$ is energetically favorable. As a consequence of this driving force for $A$ segregation, the $B$ species populate the regions deficient in $A$. In this connection, it is noted that in the studies of the codesorption of hydrogen and carbon monoxide from $\mathrm{Ni}(100)$, it was hypothesized that at low 
239

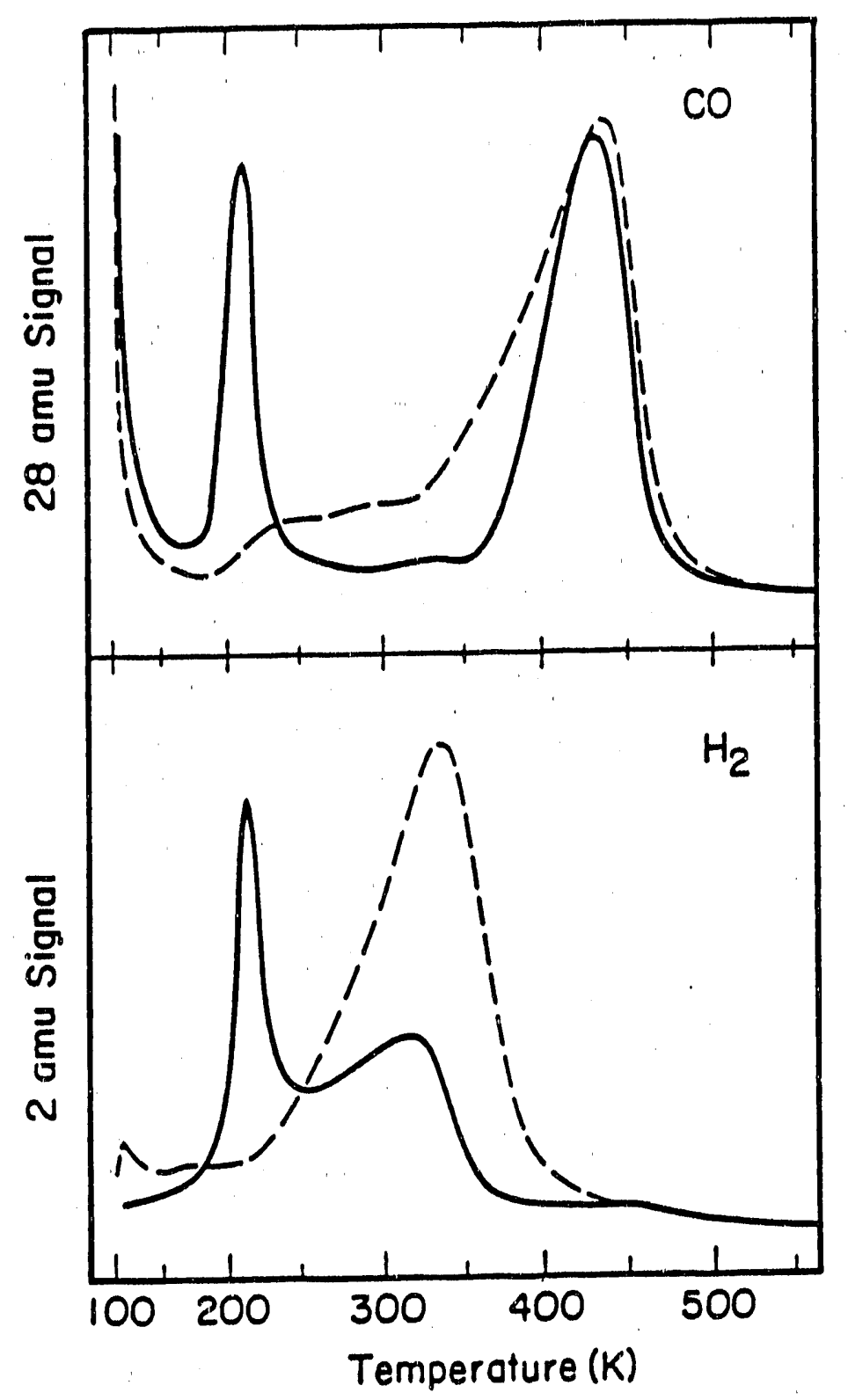

Fig. 15 Experimentally observed TPD spectra for $\mathrm{H}_{2}$ and $\mathrm{CO}$ desorption from $\mathrm{Ni}(100)$ [18]. The dashed lines are for desorption of each species adsorbed separately, and the solid lines are for desorption of both species coadsorbed. 
temperature, where $\theta H=1.0$ and $\theta c O=0.5$, adsorbed $H$ and $C O$ are intimately mixed on the surface, but as the temperature is raised and sites are vacated, the hydrogen and carbon monoxide segregate, giving rise to desorption features similar to those observed when each species is adsorbed separately $[17,18]$.

On $\mathrm{Rh}(100)$, the spectra for carbon monoxide and deuterium adsorbed separately exhiblt one and two peaks, respectively [19]. When coadsorbed, however, the carbon monoxide is only weakly affected by the presence of the deuterium, for a variety of deuterium and carbon monoxide coverages. The deuterium spectra in the presence of coadsorbed carbon monoxide, on the other hand, are shifted to lower temperatures, and new features appear. Cases II and III presented above display this kind of behavior.

\subsection{CONCLUSIONS}

The present study demonstrates that the BOC-MP method can be used successfully to represent the energetics of coadsorption of two species on well-defined metal surfaces. Monte Carlo simulations of the temperature-programmed desorption of coadsorbed species demonstrates that the number and location of the desorption peaks and the activation energy versus coverage profiles are sensitive to the relative coverages and binding energies for each species. In addition, the combined effects of the throughmetal interactions and the direct adsorbate-adsorbate interactions are seen to have important consequences on the activation energies for dnsorption and on the distribution of adsorbates on the surface. The appearance, disappearance, and shifting of spectral features are 
shown to arise from the nature of the energetic interactions between the coadsorbed species with the metal and with each other, and not from the appearance, disappearance, or modification of distinct binding sites.

The presence of strongly bound, immobile coadsorbates was found to lower the activation energy for the associative desorption of atomically adsorbed species. The magnitude of this effect increases with increasing coverage and binding strength of the coadsorbate. When molecular and atomic adsorbates of comparable activation energies for desorption are coadsorbed, either both adsorbates are strongly affected or only the atomic species is strongly affected. This dependence is sensitive to the coordination and relative binding energies of the two species. For certain circumstances, the activation energy for the desorption of the molecular adsorbate may become smaller than that for associative desorption of the atomic species. It is also found that when both adsorbates are mobile, surface segregation of the two coadsorbed species can occur, such segregation being driven by the attractive interactions between the species undergoing associative desorption.

\section{ACKNOWLDGMENT}

This work was supported by the Director, Office of Basic Energy Sciences, Chemical Sciences Division of the US Department of Energy under contract DE-AC03-76SF00098. 


\section{2}

NOMENCLATURE

\section{$A, B, A B$ Adsorbates}

a Morse potential constant $(A)$

DXY Bond dissociation energy for X-Y bond ( $\mathrm{kcal} / \mathrm{mol}$ )

$E_{d, i}$ Activation energy for desorption from the ith environment (kcal/mol)

$E_{d, A}$ Activation energy for the non-associative desorption of $A$ ( $\mathrm{kcal} / \mathrm{mol}$ )

$E_{d, A A^{\prime}}$ Activation energy for associative desorption of $A$ and $A^{\prime}$ ( $\mathrm{kcal} / \mathrm{mol}$ )

$k_{d, i^{\circ}}$ Preexponential factor for desorption from the ith environment $(s-1)$

mi Number of adsorbates bonded to the ith metal atom

$\mathrm{N}_{\mathrm{A}} \quad$ Number of adsorbates $\mathrm{A}$

$\mathrm{No}$ Number of adsorbate atoms or molecules desorbing (mol)

$\mathrm{N}_{\mathrm{s}} \quad$ Number of surface metal atoms (mol)

$n$ Number of metal atoms bonded to an adsorbate

$n_{A} \quad$ Number of metal atoms bonded to $A$

$P_{1}(1)$ Probability of desorption from the ith environment

$P_{i j}(2)$ Probability of diffusion from site $i$ to site $j$

$Q(x)$ Bond energy (kcal/mol)

Q E Equilibrium bond energy ( $\mathrm{kcal} / \mathrm{mol}$ )

QaA Heat of adsorption of $A$ in the on-top position (kcal $/ \mathrm{mol}$ )

$Q_{i}, Q_{j}$ Heats of adsorption in sites $i$ and $j(\mathrm{kcal} / \mathrm{mol})$

$Q_{A, n^{*}}$ Total heat of adsorption for $A(\mathrm{kcal} / \mathrm{mol})$

$Q_{A, n}$ Heat of adsorption of $A$ as a function of coordination ( $\mathrm{kcal} / \mathrm{mol}$ )

$Q_{A, n}(1)$ Heat of adsorption of $A$ due to through-metal interactions (kcal/mol)

$Q_{A, n}(2)$ Heat of adsorption of $A$ due to adsorbate-adsorbate interactions $(\mathrm{kcal} / \mathrm{mol})$

R Gas constant (kcal/mol K) or random number

rd Turnover frequency $\left(s^{-1}\right)$

$r$ Bond length $(A)$

ro Equilibrium bond length $(A)$

$T$ Temperature (K)

$\Delta t$ Time interval (s)

$x \quad$ Bond order 


\section{3}

$x_{i, n} \quad$ Bond order of an adsorbate bonded to $n$ metal atoms

$x_{A}$ Bond order of a metal atom bonded to an adsorbate $A$

$\alpha \quad$ Desorption order

$\beta \quad$ Heating rate $(\mathrm{K} / \mathrm{s})$

$\delta$ Direct adsorbate-adsorbate bond order

$\theta \quad$ Coverage

$\theta_{A, 0} \quad$ Initial coverage of $A$ 


\section{REFERENCES}

[1] J. Falconer and J.A. Schwarz, Catalysis Rev.-Sci. Eng. 25 (1983) 141.

[2] L.D. Schmidt, Catalysis Rev.-Sci. Eng. 9 (1974) 115.

[3] R.J. Cretanović and Y. Amenomiya, Catalysis Rev. 6 (1972) 21.

[4] D.A. King, Surface Sci. 47 (1975) 384.

[5] M.A. Morris, M. Bowker and D.A. King, in: Simple Processes at the Gas-Solid Interface, Comprehensive Chemical Kinetics, vol 19, Eds. C.H. Bamford, C.F.H. Tipper and R.G. Compton (Elsevier, Amsterdam, 1984) ch. 1.

[6] G.A. Somorjai, Chemistry in Two Dimensions: Surfaces (Cornell University Press, Ithaca, NY, 1981).

[7] H. Conrad, G. Ertl and E.E. Latta, J. of Catal. 35 (1974) 363.

[8] D.E. Ibbotson, T.S. Wittrig and W.H. Weinberg, Surface Sci. 97 (1980) 297.

[9] M.E. Bridge, C.M. Comrie and R.M. Lambert, J. of Catal. 58 (1979) 28.

[10] E.D. Williams, P.A. Theil, W.H. Weinberg and J.T. Yates, Jr., J. Chem. Phys. 72 (1980) 3496.

[11] M.P. Kiskinova and G.M. Bliznakov, Surface Sci. 123 (1982) 61.

[12] J.E. Crowell, E.L. Garfunkel and G.A. Somorjai, Surface Sci. 121 (1982) 303.

[13] J. Benziger and Fi.J. Madix, Surface Sci. 94 (1980) 119.

[14] J.E. Benziger and R.J. Madix, Surface Sci. 115 (1982) 279.

[15] J. Hrbek, J. Phys. Chem. 90 (1986) 6217.

[16] D.W. Goodman, J.T. Yates, Jr. and T.E. Madey, Surface Sci. 93 (1980) L135.

[17] B.E. Koel, D.E. Peebles and J.M. White, Surface Sci. 107 (1981) L367; 125 (1983) 709; 125 (1983) 739.

[18] J.M. White, J. Phys. Chem. 87 (1983) 915.

[19] Y. Kim, H.C. Peebles and J.M. White, Surface Sci. 114 (1982) 363.

[20] D.E. Peebles, J.A. Schreifels, and J.M. White, Surface Sci. 111(1982) 117.

[21] D.W. Goodman, T.E. Madey, M. Ono and J.T. Yates, Jr., J. of Catal. 50 (1977) 279.

[22] J.T. Yates, Jr. and T.E. Madey, J. Chem. Phys. 54 (1971) 4969.

[23] M. Textor, I.D. Gay and R. Mason, Proc. R. Soc. London A 356 (1977) 37. 
[24] D.T. Ling and W.E. Spicer, Surface Sci. 94 (1980) 403.

[25] J.J. Weimer, E. Umbach and D. Menzel, Surface Sci. 155 (1985) 132.

[26] U. Seip, I.C. Bassignana, J. Küppers and G. Ertl, Surface Sci. 160 (1985) 400.

[27] J.B. Benziger and G.R. Schoofs, J. Phys. Chem. 88 (1984) 4439.

[28] S. Sundaresan and K.R. Kaza, Surface Sci. 160 (1985) 103.

[29] D. Gupta and C.S. Hirtzel, Chem. Phys. Letters 149 (1988) 527; Surface Sci. 210 (1989) 322.

[30] S.J. Lombardo and A.T. Bell, Surface Sci. 206 (1988) 101.

[31] E. Shustorovich, Surface Sci. Rept. 6 (1986) 1; Acc. Chem. Res. 21 (1988) 183.

[32] K. Binder, Ed., Monte Carlo Methods in Statistical Physics (Springer, Berlin,1979).

[33] IMSL, Subroutine GGUBFS, Houston, TX, 1980.

[34] H.R. Han and L.D. Schmidt, J. Phys. Chem. 75 (1971) 227.

[35] F. Zaera, E.B. Kollin and J.L. Gland, Surface Sci. 166 (1986) L149.

[36] E.I. Ko, Department of Chemical Engineering, Carneige-Mellon University, unpublished results. 


\title{
Chapter V
}

Monte Carlo Simulations of the Effect of Pressure on Isothermal and Temperature-Programmed Desorption Kinetics

\begin{abstract}
A Monte Carlo simulation technique is presented for describing the adsorption, surface diffusion, and desorption kinetics of. molecules from metal surfaces. Lateral interactions between adsorbed molecules are taken into account using the Bond-OrderConservation-Morse-Potential method. The rate of desorption observed in the presence of a gas-phase species is higher than that observed in a vacuum. The increase in the apparent rate coefficient for descrption with increasing pressure can be ascribed to the effects of repulsive lateral interactions on the activation snergy for desorption. The simulated kinetics are in good agreement with the experimentally-observed kinetics for the isothermal desorption of $\mathrm{CO}$ from polycrystalline $\mathrm{Pd}$ and for the temperature-programmed desorption of $\mathrm{CO}$ from $\mathrm{Ni}(100)$.
\end{abstract}




\subsection{INTRODUCTION}

A number of studies [1-13] have shown that the rate of gas desorption from a metal surface is enhanced when desorption occurs in the presence of a finite pressure of the desorbing gas. Based on the results of isothermal studies of $\mathrm{CO}$ desorption from polycrystalline $\mathrm{Pd}$, Yamada et al. $[5,6]$ have proposed that the rate coefficient of $\mathrm{CO}$ desorption depends explicitly on the pressure of CO. This behavior was attributed to an unspecified chemical interaction in the adsorbed layer. The effects of adsorbate fluxes have also been observed in temperature-programmed desorption studies. Yates and Goodman [4] have noted that when CO desorbs from $\mathrm{Ni}(100)$ in the presence of gas-phase $\mathrm{CO}$, the positions of the desorption peaks observed in vacuum shift to lower temperatures and new, low-temperature features appear. The authors ascribed these effects to the influence of lateral interactions on the activation energy for desorption.

The possibility of collision-induced desorption has also been considered. Yamada et al. [5] found that when CO desorption was carried out in a background of Ar the rate of desorption was identical to that observed in vacuum. Similar results were obtained by Yates and Goodman [4], who found that the presence of $\mathrm{N}_{2}$ had no effect on the TPD spectrum of CO. More recently, Ceyer and coworkers [14-17] have observed that the rate of $\mathrm{CO}$ desorption from a $\mathrm{Ni}(141)$ surface can be enhanced by a flux of mono-energetic $\mathrm{Ar}$ atoms but only when the translational kinetic energy of the $\mathrm{Ar}$ atoms exceeds $60 \mathrm{kcal} / \mathrm{mol}$. Taken together, these observations 


\section{8}

suggest that for gas temperatures normally encountered in catalysis $(300-1300 \mathrm{~K})$, collision-induced desorption is not significant.

Here, we present a theoretical analysis of the influence of molecular adsorption on the desorption of adsorbed species. Our approach is to use a Monte Carlo simulation technique which accounts for the elementary surface processes of adsorption, surface diffusion, and desorption. Also included in the model is the influence of lateral interactions between adsorbed species. We compare the results of the simulations with the experimental data for the isothermal desorption of $\mathrm{CO}$ from polycrystalline $\mathrm{Pd}[5,6]$ and the temperature-programmed desorption of $\mathrm{CO}$ from $\mathrm{Ni}(100)$ [4]. The model calculations demonstrate that the apparent pressuredependence of the desorption kinetics is a direct consequence of lateral interactions between adsorbed molecules.

\subsection{THEORY}

Adsorbate molecules are assumed to participate in three elementary processes: adsorption, diffusion, and desorption. While on the surface of the metal, molecules are assumed to occupy welldefined sites on a single-crystal lattice. The coordination of the adsorbate is defined by the composition of the metal and the adsorbate. Since the local occupancy of adsorption sites varies with position on the surface and adsorbate-adsorbate interactions are known to affect the heat of adsorption, the dynamics of adsorption and desorption are taken to be site-specific. In view of the inhomogeneous distribution of adsorbates, Monte Carlo techniques 
are used to represent the effects of adsorption, diffusion, and desorption on the net rate of adsorption or desorption of adsorbates. The probability of adsorption on a vacant site $i$ in the time interval $\Delta t$ can be defined as (see Appendix for the derivation of eq. 1):

$P_{i}^{a}=S_{0} P a_{s} /\left(2 \pi m k_{b} T_{g}\right)^{1 / 2} \Delta t=S_{0} F \Delta t$

where $S_{0}$ is the sticking coefficient at zero coverage, $P$ is the pressure, as is the area per site, $m$ is the mass of an adsorbate, $k b$ is Boltzmann's constant, and $T_{g}$ is the temperature of the gas phase. The factor $F$ on the far right-hand side of eq. 1 is the flux of adsorbates per site. The total rate of adsorption, expressed as a turnover frequency based on the number of surface metal atoms, $N_{s}$, is given by

$$
\begin{aligned}
r_{a} & =\sum_{i} N_{a, i} /\left(N_{s} \Delta t\right) \\
& =N_{a} /\left(N_{s} \Delta t\right)
\end{aligned}
$$

The probability of desorption from site $i$ in the time interval $\Delta t$ can be represented as $P_{i}^{d}=v_{i} \exp \left[-E_{d, i} / k_{b} T\right] \Delta t$

where $v_{i}$ is the frequency factor for desorption and $E_{d, i}$ is the activation energy for desorption. The total rate of desorption from all local environments is simply

$$
\begin{aligned}
r_{d} & =\sum_{i} N_{d, i} /\left(N_{s} \Delta t\right) \\
& =N_{d} /\left(N_{s} \Delta t\right)
\end{aligned}
$$

Since the activation energy for surface diffusion is $10-15 \%$ of 
that for desorption, whereas the frequency factors for the two processes are comparable, surface diffusion is expected to occur much more rapidly than desorption. As a consequence, it is assumed that the spatial distribution of adsorbate molecules will never be far from that corresponding to equilibrium. Perturbations to the equilibrium distribution caused by the adsorption or desorption of molecules are eliminated by repositioning the adsorbed molecules in accordance with Kawasaki statistics. The probability that an adsorbate on site $i$ moves to an adjacent site $j$ is given by [18]: $P_{i j}=\frac{\exp \left[-\left(Q_{i}-Q_{j}\right) / k_{b} T\right]}{1+\exp \left[-\left(Q_{i}-Q_{j}\right) / k_{b} T\right]}$

where $Q_{i}$ and $Q_{j}$ are the heats of adsorption for an adsorbate at sites $i$ and $j$, respectively. Equation 5 weights diffusional jumps according to the magnitude of $\left(Q_{i}-Q_{j}\right)$. It should be noted that if $Q_{i}=$ $Q_{j}$, then $P_{i j}(2)=0.5$, and the two sites have equal probability of occupancy.

To complete the descriptions of the probabilities of desorption and diffusion given by eqs. 3 and 5 , the values of $Q_{i}$ and $E_{d, i}$ appearing therein must be determined. Our approach is to use the Bond-Order-Conservation-Morse-Potential (BOC-MP) method developed by Shustorovich $[19,20]$ because it allows us to account for the effects of local site occupancy without the introduction of arbitrarily assigned energy parameters. Since the BOC-MP method has been discussed extensively elsewhere [19-22], only those relationships required for this study are summarized here.

The heat of adsorption for a molecular adsorbate $A B$ coordinated via atom $A$ to $n$ metal atoms can be approximated by 
$Q_{A B, n}=\frac{Q_{O A}^{2}}{D_{A B}+Q_{O A} / n}$

where $D_{A B}$ is the gas-phase dissociation energy for the $A-B$ bond and $Q_{O A}$ is the heat of adsorption of $A$ in the on-top position.

Equation 6 is appropriate for isolated adsorbate molecules. When the adsorbate coverage increases, situations arise in which more than one adsorbate is coordinated with a single metal atom. In this case, the heat of adsorption is given by $\dot{Q}_{A B, n}=\sum_{i=1}^{n} \frac{Q_{A B, n}}{n m_{i}}\left(2-1 / m_{i}\right)$

where $\mathrm{mi}$ is the number of adsorbates bonded to the ith metal atom. The expression for $Q_{A B, n^{*}}$ can be used to determine the activation energy for desorption. For the non-associative desorption of a molecule, the activation energy is given by the heat of adsorption, $E_{d}=Q_{A B, n^{*}}$. Implicit in this relationship is that adsorption is non-activated.

The energy and probability formulations described above were incorporated into a Monte Carlo algorithm for simulating isothermal and temperature-programmed kinetic experiments. The metal surface was represented by a 100 by 100 array of numbered sites, and periodic boundary conditions were used to eliminate edge effects. When an initial coverage was required to begin a simulation, molecules were placed randomly on the lattice to achieve an initial coverage, $\theta^{\circ}$.

Each simulation was divided into a sequence of Monie Carlo steps (MCS) and each MCS was subdivided into three segments 
corresponding to the adsorption, diffusion, and desorption of molecules. The time interval for each MCS, $\Delta t$, was chosen sufficiently small so that the change in surface coverage during each segment of an MCS was less than ten percent of the saturation coverage. Specifying $\Delta t$ in this manner is necessary for eqs. 1 and 3 to be valid representations of the probabilities of adsorption and desorption, respectively, (see Appendix).

For the adsorption segment of the first MCS, the temperature was initialized at $T_{0}$ and taken to be constant at this value for the time interval $\Delta t$. During this interval, molecules were adsorbed on the surface in the following manner. A surface site was selected in a random fashion. If the site were unoccupied, the probability of adsorption was calculated using eq. 1. The calculated adsorption probability was then compared with a random number, $R(0<R<1)$. If $\mathrm{R}<\mathrm{Pia}$, the adsorbate was placed on the lattice, and $\mathrm{Na}_{\mathrm{a}}$ in eq. 2 was incremented by one. Conversely, if $R \geq P_{j} a$, the site remained unoccupied. The number of unoccupied sites visited during an MCS was $\left[S(\theta) / S_{0}\right] N_{s}$, where $S(\theta)$ is the sticking coefficient. The form of $S(\theta)$ used for specific simulations is discussed in the next section. After completing the sampling of the surface, the rate of adsorption for this segment of the MCS was calculated using eq. 2 and the surface coverage was updated to account for the adsorption of $\mathrm{Na}_{\mathrm{a}}$ molecules.

During the second segment of the first MCS, a redistribution of the adsorbates was carried out to account for the effects of surface diffusion. A surface site and an adjacent site were chosen at random. If the surface site were occupied and the nearest-neighbor 
site were vacant, a probability of diffusion was calculated using eq. 5. Q was taken as the heat of adsorption in the initial site, and $Q$ ] was taken as the heat of adsorption in the final site. The calculated value of $P_{i j}$ was then compared with a random number, $R(0<R<1)$. If $R<P_{i j}$, the adsorbate was moved from the initial site, $i$, to the adjacent site, $j$. Conversely, if $R \geq P_{i j}$, movement of the adsorbate was not allowed. A sufficient number of surface visitations was allowed to attain an equilibrium distribution of adsorbates on the surface. The attainment of equilibrium was defined by a constant average heat of adsorption.

During the final segment of the first MCS, the desorption of adsorbates was treated in the following manner. A surface site was selected in a random fashion. If the site were occupied, the probability of desorption was calculated using eq. 3 . The local. activation energy for desorption was calculated using eq. 7. The value of $P_{i} d$ was then compared with a random number, $R(0<R<1)$. If $R<P_{d} d$, the adsorbate was removed from the lattice, and $N_{d}$ in eq. 4 was incremented by one. Conversely, if $R \geq P$ d the site remained occupied. The number of occupied sites visited during an MCS was $\theta N_{s}$. After completing the sampling of the surface, the rate of desorption for this MCS was calculated using eq. 4 and the surface coverage was updated to account for the desorption of $\mathrm{Nd}$ molecules.

The adsorption, diffusion, and desorption segments described above constitute a Monte Carlo Step (MCS). As noted above, the time interval associated with an MCS is $\Delta t$. For simulations in which the surface cemperature was maintained constant, a new MCS was begun by incrementing the time by $\Delta t$. For temperature-programmed 


\section{4}

desorption simulations, the temperature was increased by the increment $\beta \Delta t$, where $\beta$ is the heating rate. A new MCS was then carried out next. This process was repeated for either a specific length of time or until a desired temperature was reached. The results of the simulations were obtained as plots of coverage versus time or as the rate of desorption versus temperature. All of the calculations described above were carried out on an IBM 3090 computer. Random numbers were generated by the IMSL linear congruential number generator GGUBFS [23].

\subsection{RESULTS AND DISCUSSION}

3.1 Isothermal desorption of $\mathrm{CO}$ from $\mathrm{Pd}$

The desorption of $\mathrm{CO}$ from polycrystalline $\mathrm{Pd}$ has been investigated by Yamada et al. $[5,6]$ with the following type of experiment. At $t=0, C 180$ at a pressure $P$ is adsorbed onto a clean Pd surface held at a surface temperature $T_{s}$. At $t=t_{1}$, the gas-phase is quickly switched from $\mathrm{C} 180$ to $\mathrm{C} 160$ while maintaining a constant pressure. At $t=t_{1}+t_{2}$, the surface is flashed to high temperature. The resulting TPD spectra resulting are integrated to determine the coverages of $C^{18 O}$ and $C^{16 O}, \theta\left(C_{18 O}\right)$ and $\theta\left(C^{16 O}\right)$, and the total coverage, $\theta T=\theta\left(C^{180}\right)+\theta\left(C^{160}\right)$. Repetition of this procedure for a series of $t_{1}$ and $t_{2}$ generates plots of coverage versus time. Figures 1a-d show examples of the data obtained by Yamada et al. [6] with this approach. As seen in the figures, the total coverage initially rises with time until it reaches its equilibrium value for a given pressure. As the pressure is increased, higher equilibrium coverages 


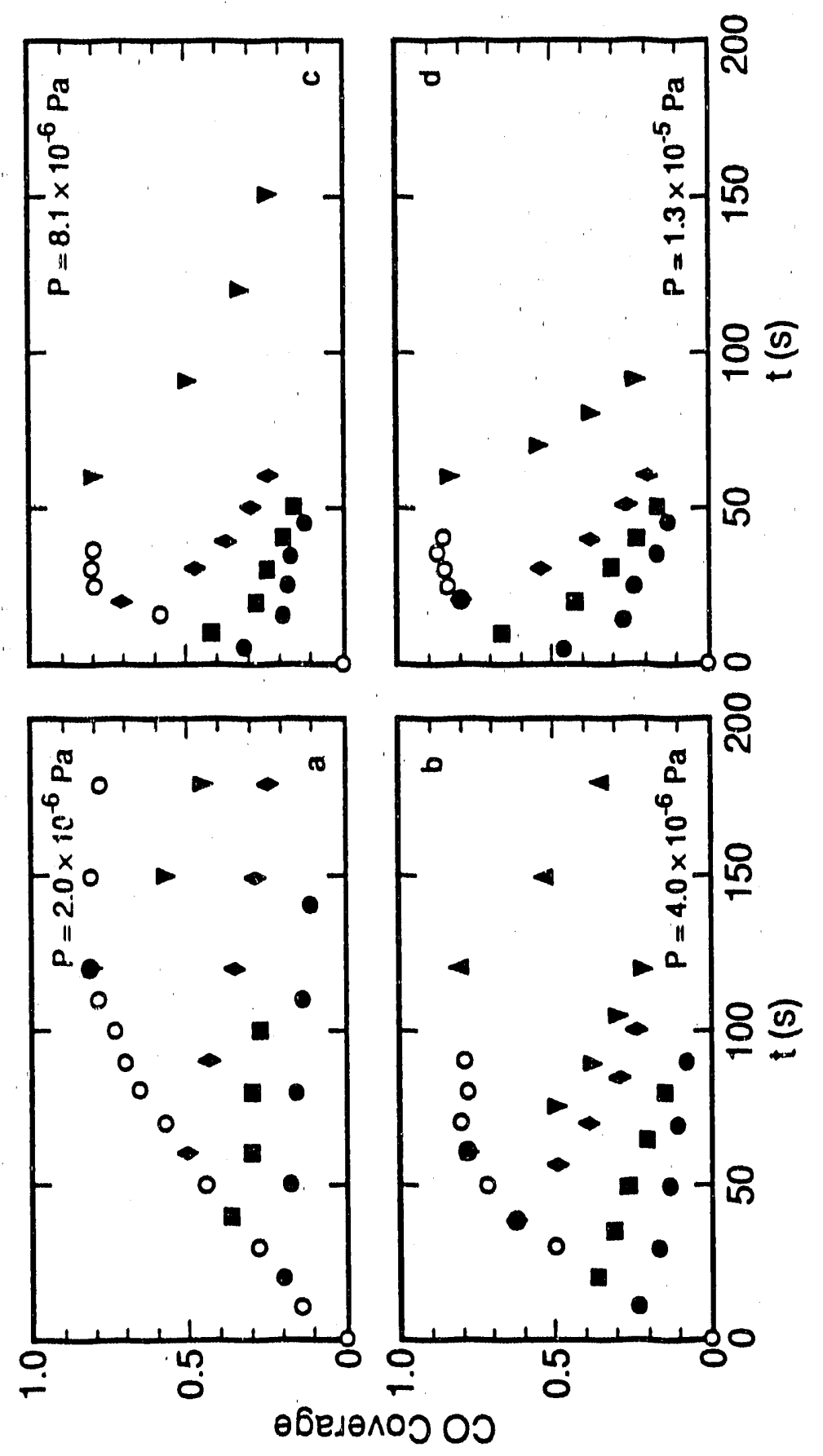

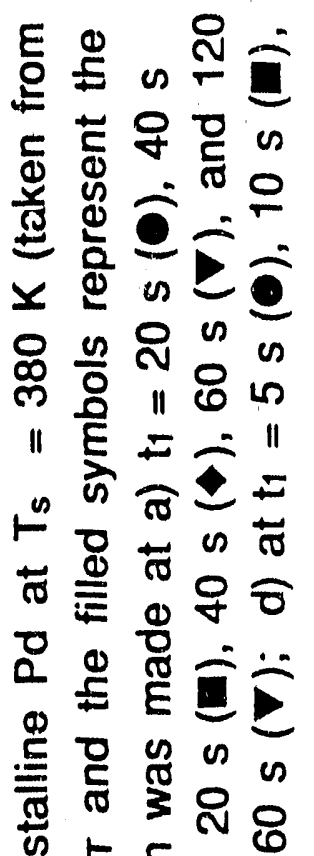

ํํㅇㅎํ워ำ

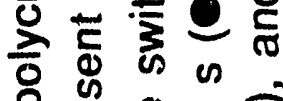

뽕요울

ธ웧ㅇㅇㅇ " w

象

乌 는

कूँ

品 0 品

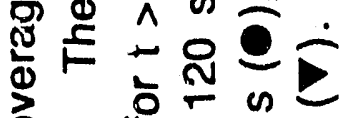

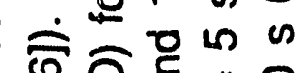

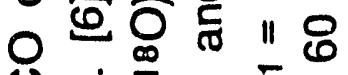

U एं

등

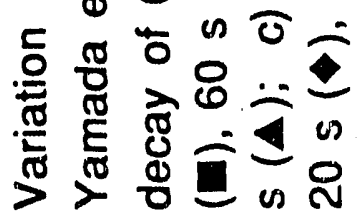

i̊n 
are achieved in shorter periods of time. Also shown in figs. 1a-d are the curves of the decay of $\theta\left(C^{180}\right)$ for $t>t_{1}$. The rates of $\theta\left(C_{180}\right)$ decay are seen to depend on the pressure and the coverage at which the isotope switch is made.

Yamada et al. [5] obtained apparent rate coefficients for the desorption of $\mathrm{C} 180$ by fitting the decay curves of $\theta\left(C_{180}\right)$ to single exprnential functions of the form $\theta\left(C_{180}\right)=\theta^{\circ}\left(C_{180}\right) \exp \left[-k_{d}\left(t-t_{1}\right)\right]$, where $\theta^{\circ}\left(C_{180}\right)$ is the value $\theta\left(C_{180}\right)$ at $t=t_{1}$. The initial rate of $C_{180}$ desorption at the time of the isotope switch $t=t_{1}$ (i.e., the product $\left.\theta^{\circ}\left(C^{180}\right) k_{d}\right)$ was found io be approximately linear with coverage. The rate coefficients determined from the slopes of the initial rate versus coverage curves were then plotted versus $P$. As seen in fig. 2 , the rate constants $\mathrm{Kd}_{d} \mathrm{P}$ exhibit an apparent linear dependence on the pressure. Based on this result, Yamada et al. [5] postulated that the rate of desorption is given by the expression $r_{d}=k_{d}^{\prime} \theta+k^{\prime \prime}{ }_{d} P^{\alpha} \theta=k_{d}^{P} \theta$

where $\mathrm{kd}^{\circ}$ is the desorption rate coefficient when $P=0, \mathrm{kd}^{\prime \prime}$ is the desorption rate coefficient which accounts for the effects of pressure when $P \neq 0$, and $\alpha$ is the power law exponent describing the dependence on pressure. It should be noted that while Yamada et al. [5] assumed $k_{d}$ ' to be coverage independent, their plots of $r d$ versus $\theta$ for the case when $P=0$ are parabolic in shape, indicating that $\mathrm{Kd}^{\prime}$ increases with increasing coverage.

In the simulations of the experiments of Yamada et al. [5,6], CO molecules were assumed to occupy both types of bridge sites on a $\operatorname{Pd}(100)$ surface $[26,27]$. A maximum of two $C O$ molecules was 


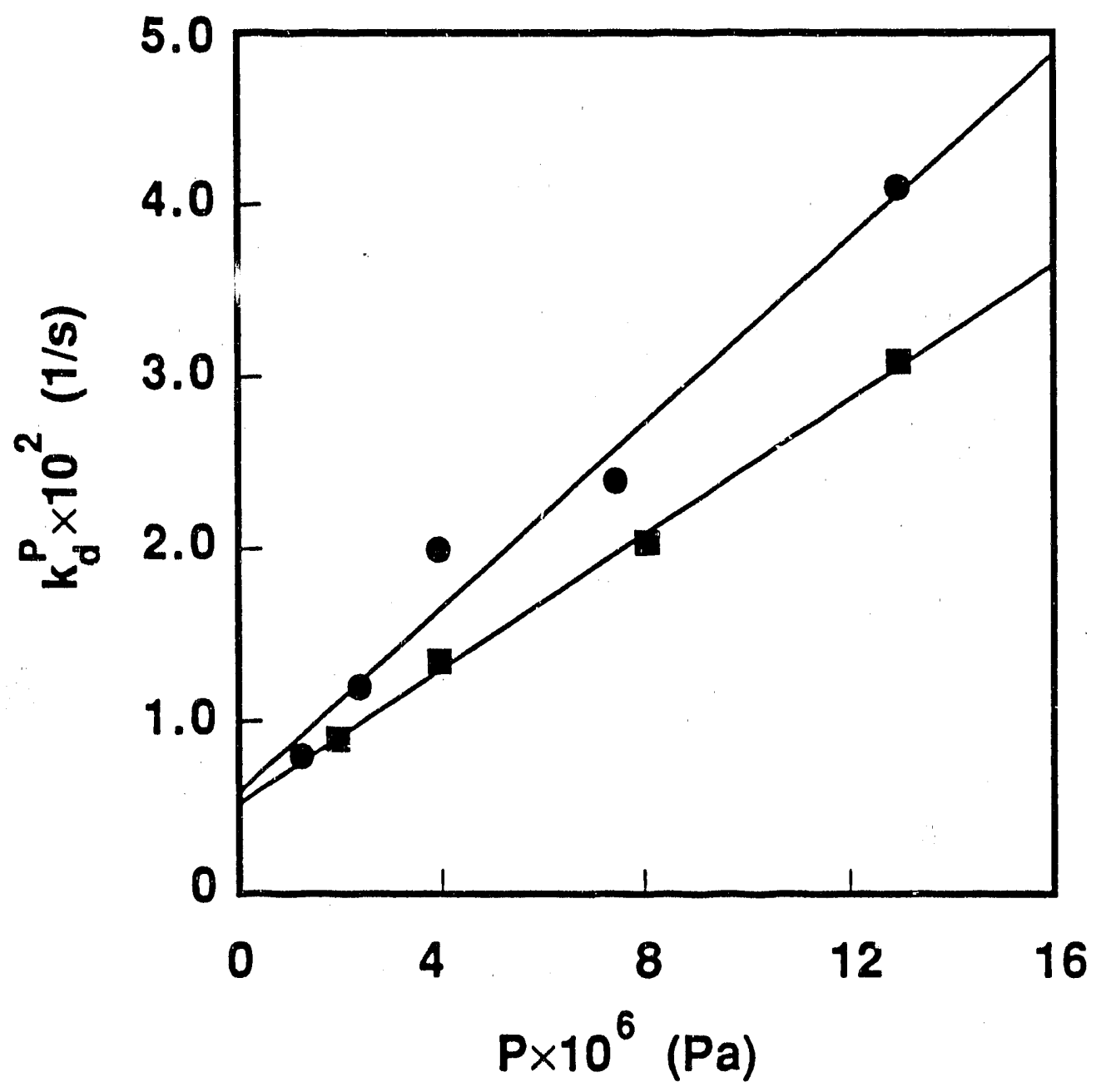

Fig. 2 Variation of $k_{d} P$ versus $P$. The points designated by $(0)$ are from the analysis of ixperimental data presented by Yamada et al. [5] and the points designated by ( $\mathbf{a})$ are the values obtained from the Monte Carlo simulations. 
allowed to be bonded to each metal atom, and because of steric constraints, the two molecules had to be located at $180^{\circ}$ from each other relative to the shared metal atom. Exclusion of two $\mathrm{CO}$ molecules located at $90^{\circ}$ relative to the shared metal atom is justified by the fact that in such a case the CO-CO distance would be $1.94 \mathrm{~A}$, whereas the hard-sphere diameter for $\mathrm{CO}$ is approximately $2.7 \AA$ [24]. The saturation coverage of $C O$ was taken to be $6.4 \times 10.14$ molecules $\mathrm{cm}^{-2}$, the value reported by Yamada et al. [5]. This coverage corresponds to one CO molecule per two metal atoms for a Pd(100) surface.

The sticking coefficient for adsorption and its dependence on coverage were taken from the work of Yamada et al. [5]. In this study, the authors concluded that the function $S(\theta)$ could be described by a precursor model [25]. The specific form of $S(\theta)$ used for the simulations was

$$
S(\theta)=S_{0}\left(1+\frac{\theta_{T}}{1-\theta_{T}} K\right)^{-1}
$$

where $S_{0}$ is the sticking coefficient at zero coverage and $K=$ $k_{d}{ }^{*} /\left(k_{d}{ }^{*}+k_{a}{ }^{*}\right)$. The rate constant $k_{d}{ }^{*}$ is the desorption rate constant from extrinsic precursor states, and $k_{d}{ }^{\circ}$ and $k_{a}{ }^{*}$ are the rate constants for desorption and adso: stion, respectively, from intrinsic precursor states. The values of $S_{0}$ and $K$ ara 0.88 and 0.05 , respectively.

To calculate desorption probabilities, the value of $v$ in eq. 3 was taken as $1016 \mathrm{~s}-1$. This value fall within the range reported for $\mathrm{CO}$ on Pd surfaces $[26,28]$. The activation energy for desorption was calculated from en. ?. The value of Qco,n in this equation was taken 
as $31.5 \mathrm{kcal} / \mathrm{mol}$, a value that lies within in the range of $30-40$ $\mathrm{kcal} / \mathrm{mol}$ reported in the literature for $\mathrm{CO}$ on $\mathrm{Pd}$ surfaces $[24,26,28,29]$.

The results of the simulations are shown in figs. 3a-d. The equilibrium coverage is seen to increase with increasing $\mathrm{CO}$ pressure whereas the time required to reach the equilibrium coverage decreases. Comparison of figs. 3a-d with figs. 1a-d indicates that the general trends in the simulations resemble those observed in the experimental data. Exact agreement between the simulations and the experiments was not sought, since the simulations were carried out assuming a $\operatorname{Pd}(100)$ surface, whereas the experimental data were obtained on polycrystalline Pd.

Nevertheless, the level of agreement between the experimental and simulated data is sufficiently high to suggest that the simulations capture the essential features of the experiments.

The curves of $\mathrm{CO}$ coverage versus time presented in fig. 3a-d were analyzed according to the method used by Yamada et al. [5]. As shown in fig. 2, treating the data in this fashion leads to a linear pressure dependence of the apparent rate coefficient for desorption. Both the slope and intercept of the dependence of $k_{d} P$ on pressure determined from the simulations agrees rather well with the values reported by Yamada et al. [5].

Before addressing the origin of the apparent pressure dependence of $k_{d} P$, it should be noted that the isotope switch from C 180 to $\mathrm{C} 16 \mathrm{O}$ is made under two sets of conditions: before the total coverage is at its equilibrium value and after the total coverage has reached its equilibrium value. When the switch is made before the 

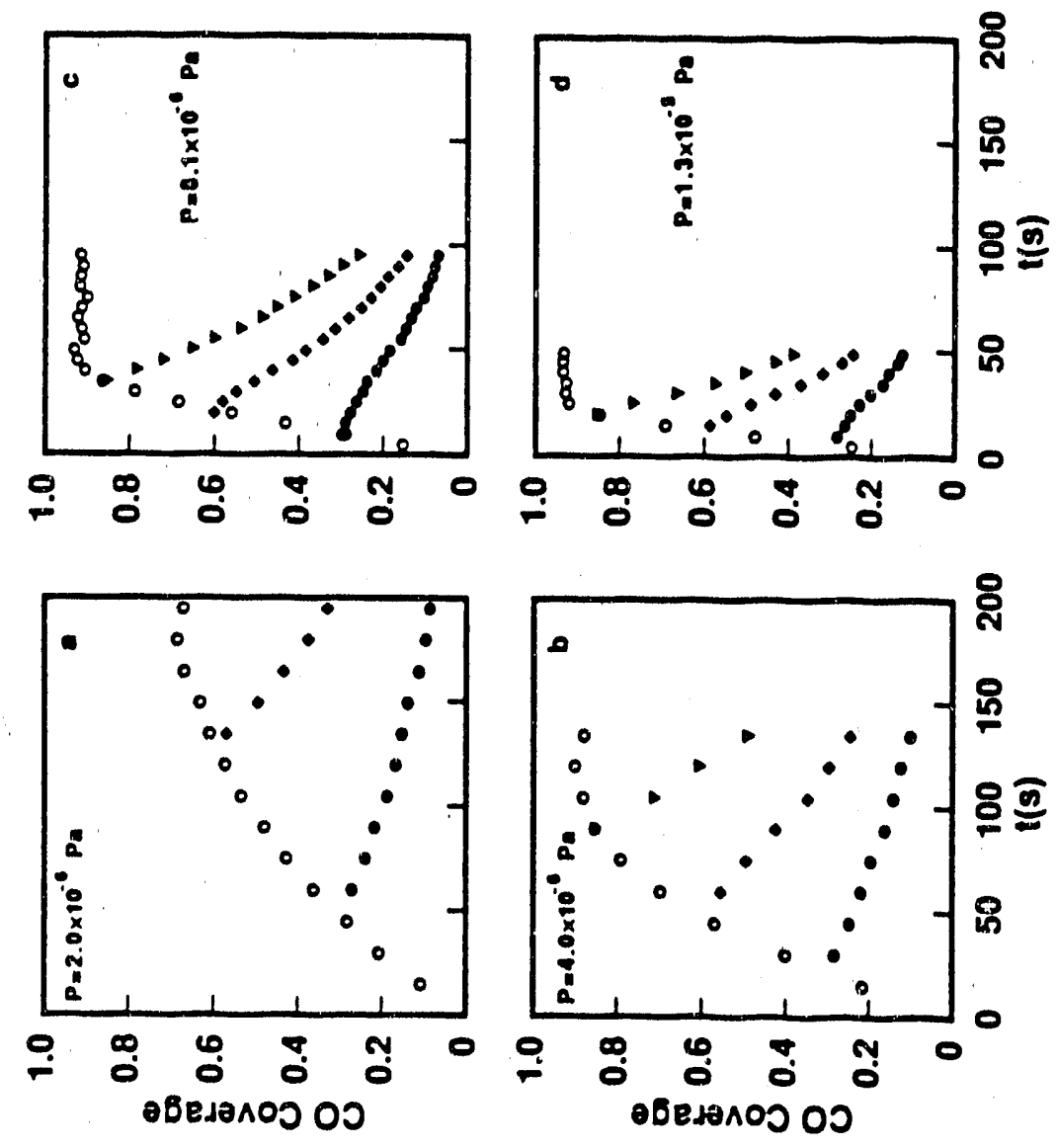

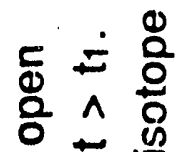

톨 욜

담

$\Upsilon$ के

잉

s $0 \dot{1}$

$\mapsto>0$

정

용 융 웅

Е 0

둔 응

댕요

○。

튼 "

능 융

कू हैं

$>$ के

品 元

『

क्ञ

ठㅇ Е

○

.

동 冚

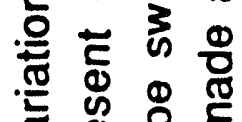

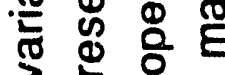

훙ㅎㅇㅇㅝ

离

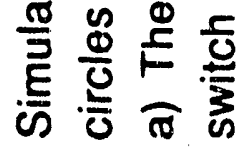

$\infty$

i⿺辶一 
equilibrium coverage is reached, the desorption of C180 occurs while the total coverage is increasing. The rate at which the total coverage increases is proportional to the gas pressure. When the switch is made after the equilibrium coverage is reached, the desorption of $\mathrm{C} 180$ occurs at a constant total coverage which is proportional to the pressure. Thus, in both cases, the pressure determines the total coverage trends.

Figure 4 shows that the average activation energy of the desorbing $C 180$ molecules decreases from $31.5 \mathrm{kcal} / \mathrm{mol}$ at $\theta T=0.35$ to $27.5 \mathrm{kcal} / \mathrm{mol}$ at $\theta \mathrm{T}=0.90$. As a consequence, changes in $\theta \mathrm{T}$ due to the effects of pressure will influence the activation energy for desorption. It follows, therefore, that the pressure dependence of $K_{d} P$ observed in fig. 2 can be ascribed to the effects of lateral interactions on the activation energy for CO desorption, which, in turn, can be related back to changes in $\theta T$ with pressure.

It is interesting to compare the results of the present model with the continuum model presented earlier by Zhdanov [30]. In Zhdanov's model, the rates of adsorption and desorption of each isotopically labeled form of $\mathrm{CO}$ are given by $r_{a}=F_{j} S\left(\theta_{T}\right)$

and

$r_{d}=v \exp \left[-E_{d}\left(\theta_{T}\right) / k_{b} T\right] \theta_{j}$

where $F_{j}$ is the flux of isotope $j$ to the surface and $\theta_{j}$ is the coverage of the surface by isotope $j$. The function $S(\theta T)$ is given by eq. 9 with $S_{0}=0.90$ and $K=0.10$. The coverage dependence of the activation energy for desorption is given by 


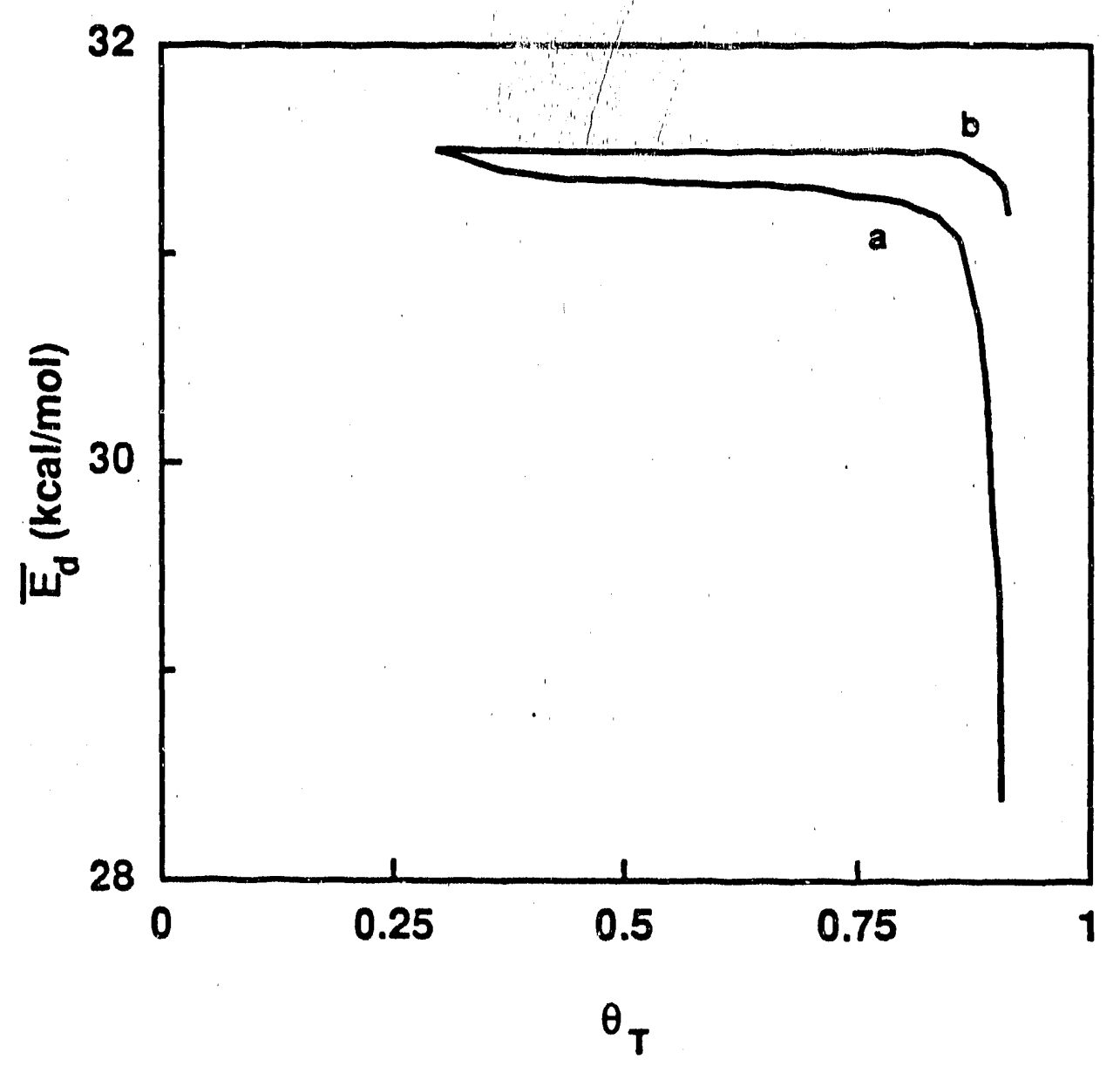

Fig. 4 Variation in the average value of $E_{d}$ as a function of $\theta T$. Curve $a$ is for the C180 molecules which desorb and curve $b$ is for all of the $\mathrm{C} 180$ molecules on the surface. The results shown are for simulation presented in fig. $3 \mathrm{c}$ for the case where the isotope switch is made at $\theta\left(C_{180}\right)=$ 0.30 . 
$E_{d}\left(\theta_{T}\right)=E_{d}^{0}-\phi \theta_{T}^{2}$

where $E_{d^{\circ}}(=39 \mathrm{kcal} / \mathrm{mol})$ is the activation energy at zero coverage and $\phi(=12 \mathrm{kcal} / \mathrm{mol})$ is the coefficient describing the magnitude of the coverage dependence. The value of $v$ in Zhdanov's model is 1016 s.1. The variation in $\theta_{j}$ with time is then dictated by $\frac{d \theta_{1}}{d t}=r_{a} \cdot r_{d}$

Equations $10-13$ were solved numerically to obtain coverage versus time profiles. As seen in fig. 5 , the decay curves of $\theta\left(C_{180}\right)$ determined from Zhdanov's model exhibit a flat induction period before decreasing. This pattern is inconsistent with that observed in the experiments of Yamada et al. [6] (see fig. 1) and in the Monte Carlo simulations reported here (see fig. 3).

To determine whether the observed induction period is a consequence of the manner is which the curves of $\theta\left(C^{180}\right)$ versus time are calculated or the manner in which the activation energy dependence on coverage is represented, Monte Carlo simulations were conducted in which the activation energy for desorption appearing in eq. 3 was calculated from eq. 12. The resulting coverage versus time profiles are shown in fig. 5. The Monte Carlo simulations are seen to be in close agreement with those obtained by numerical solution of eqs. 10-13, and both methods of simulation produce an induction period in the decay curves of $\theta(\mathrm{C} 180)$. [The deviations of the Monte Carlo results from the numerical solution are attributable to the effects of finite step sizes and the approximation made in deriving the probabilities of adsorption and 


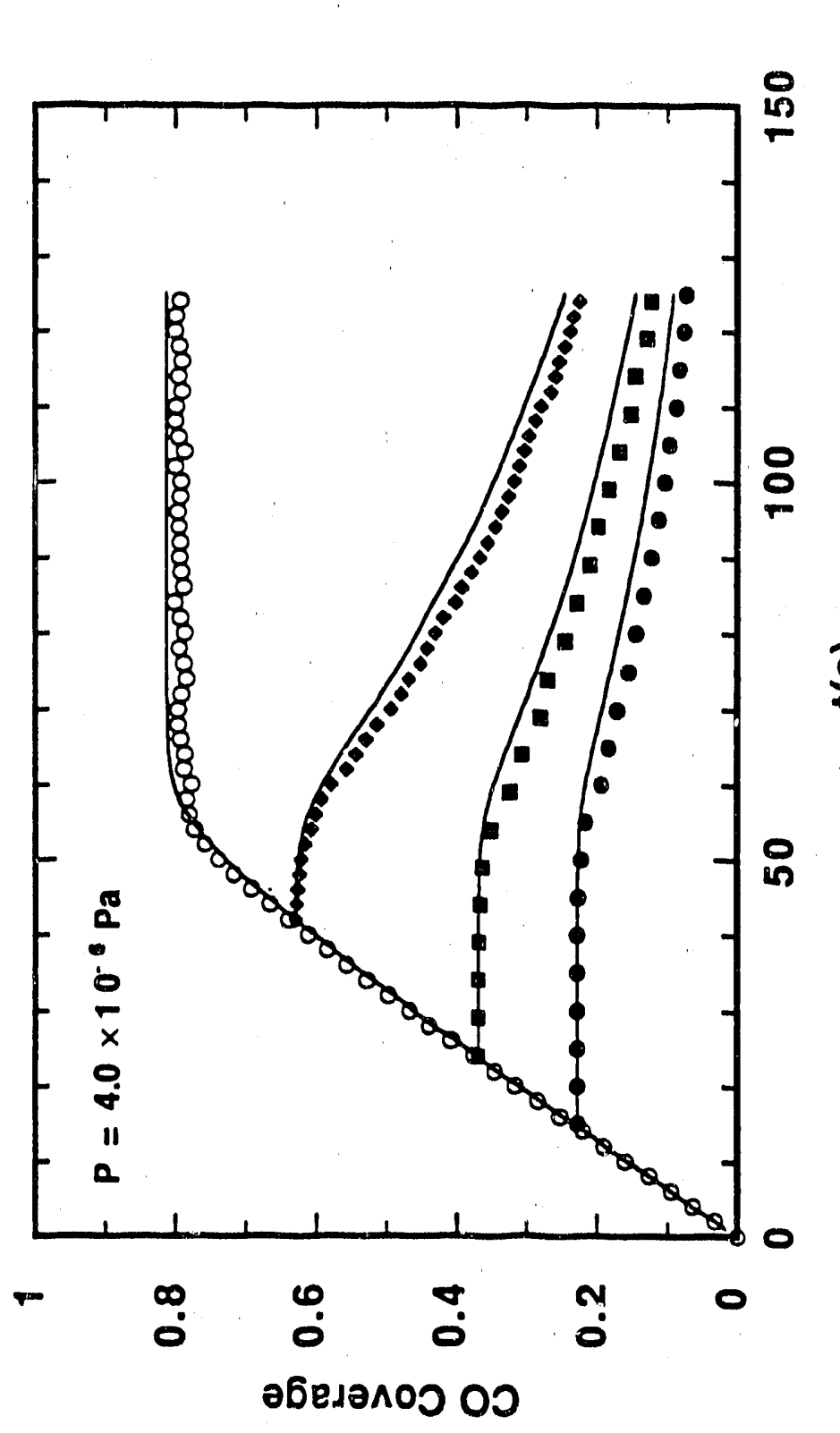

$\stackrel{\oplus}{F}$

क्ष

ब흉

क के

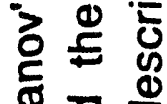

즁 은

六

등ำ 는

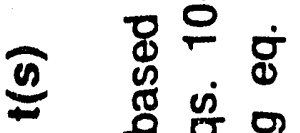

总

응

윤동

is 总

흥 홍

톨 은 은

๑

总

巳

品=

离 总

0 형

엉인

용

노웛

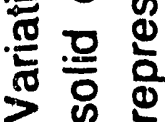

in

iํํㄴ 
desorption (see Appendix).] It is, therefore, apparent that the appearance of an induction period can be traced to the use of eq. 12 for describing the effects of adsorbate coverage on the activation energy for desorption.

In contrast to Zhdanov's model, which makes use of the mean field (Bragg-Williams) approx nation, the model reported here accounts explicitly for the local coverage dependence of $E_{d}$, through eq. 7. An important consequence of this feature is that the average activation energy of desorbing $\mathrm{CO}$ molecules is lower than the average activation energy for desorption calculated for all molecules present on the surface at a given coverage. This print is well illustrated in fig. 4 , where curve a represents the average activation energy of desorbing $\mathrm{CO}$ molecules and curve $b$ represents the activation energy for desorption averaged over all adsorbed $\mathrm{CO}$ molecules. Figure 4 also shows that the coverage dependence of curve $a$ is much stronger than that for curve $b$. As discussed in ref. [21], the difference between curves $a$ and $b$ can be ascribed to the fact that desorption occurs preferentially from sites having a local coverage higher than the average. It is exactly this aspect of the present model that results in the absence of an induction period in the simulations of the decay in $\mathrm{CO}$ coverage versus time shown in fig. 3.

Finally, we note that the shape of curve $b$ in fig. 4 closely resembles that determined from the analysis of equilibrium isobars and isosteres for $\mathrm{CO}$ on $\mathrm{Pd}(100)$ surfaces $[24,26]$. This indicates that the BOC-MP representation of the dependence of $E_{d}$ on $\theta$ provides a physically correct description. 
3.2 Temperature-programmed desorption of $\mathrm{CO}$ from $\mathrm{Ni}(100)$

Yates and Goodman [4] have examined the desorption of $\mathrm{C}^{180}$ from $\mathrm{Ni}(100)$ in the presence and in the absence of a flux of $\mathrm{C} 16 \mathrm{O}$. The TPD spectra for $\mathrm{C}_{180}$ desorbing in the absence of a $\mathrm{C} 16 \mathrm{O}$ flux are shown in fig. $6 \mathrm{a}$ as a function of gas exposure. The high-temperature $\beta$ states are seen to fill first followed by the low-temperature $\alpha$ states. The principal feature in the spectrum is the $\beta_{2}$ peak at $425 \mathrm{~K}$.

The effect of desorbing a saturated surface layer of $C^{180}$ into a flux of $\mathrm{C} 16 \mathrm{O}$ is seen in fig. $6 \mathrm{~b}$. The presence of the $\mathrm{C} 160$ flux is seen to shift the TPD spectra of $C 180$ to lower temperatures relative to the spectrum observed in the absence of the beam flux. In addition, the integrated intensity of the $\alpha_{1}$ and $\alpha_{2}$ features is larger than that observed in the absence of the C160 flux.

Simulations of the experiments of Yates and Goodman [4] were conducted in the following manner. CO molecules were allowed to occupy both types of bridge sites on a $N i(100)$ surface with a maximum of 2 molecules per metal atom. As described in the previous section, the bonding of $\approx$ jorbates $90^{\circ}$ to each other on the shared metal atom was not allowed. It should be mentioned that HREELS studies of $\mathrm{CO}$ adsorption on $\mathrm{Ni}(100)$ have shown that $\mathrm{CO}$ occupies both bridge and on-top sites and that the relative occupancy of the two types of sites is dependent on the temperature and coverage [31]. This work demonstrated that at high coverage, bridge-bonding of $\mathrm{CO}$ predominates. For this reason, $\mathrm{CO}$ molecules were assigned to bridge sites at all coverages. The coverage in 


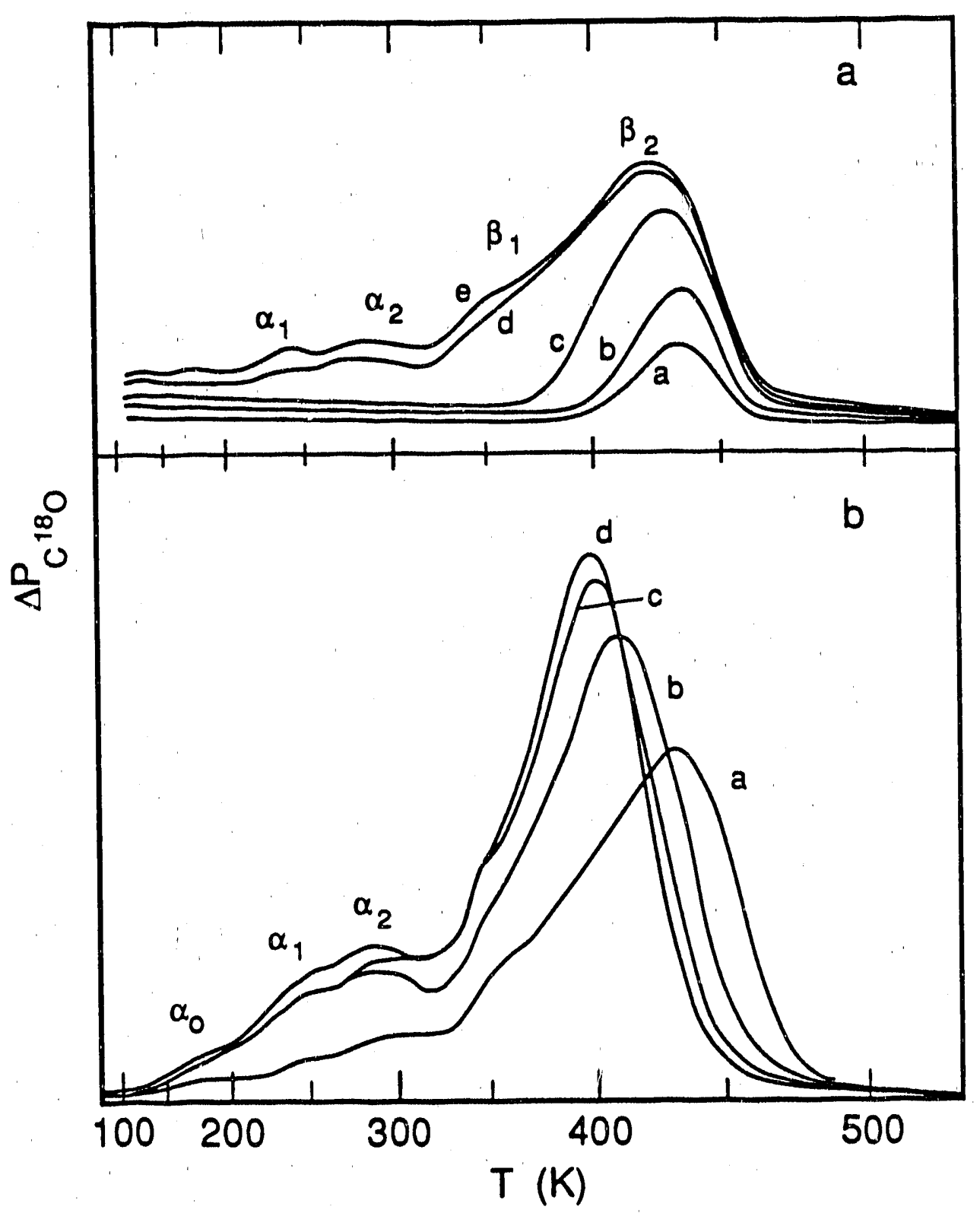

Fig. 6 TPD spectra of $\mathrm{C}^{180}$ desorption from $\mathrm{Ni}(100)$ reported by Yates and Goodman [4]. a). Desorption in the absence of gas-phase $C_{160}$. For curve $a$, the $C 180$ exposure is $0.4 \mathrm{~L}$; for curve b, $0.8 \mathrm{~L}$; for curve $c, 1.5 \mathrm{Li}$ for curve d, $3.0 \mathrm{~L}$; and for curve e, $8.0 \mathrm{~L}$. b) Desorption of $C 180$ (4.0 L dose) in the presence of gas-phase $\mathrm{C} 160$. For curve $a$, the $\mathrm{C} 160$ flux is $0 \mathrm{~cm}^{2} \mathrm{~s}^{-1}$; for curve b, $4.1 \times 1014 \mathrm{~cm}^{2} \mathrm{~s}-1$; for curve c, $8.3 \times 1014 \mathrm{~cm}^{2} \mathrm{~s}^{-1}$; and for curve d, $14.5 \times 1014 \mathrm{~cm}^{2}$ s.1. 
these simulations was defined as the number of $\mathrm{CO}$ molecules divided by the number of surface metal atoms.

Equation 9 was used to represent the sticking coefficient for $\mathrm{CO}$ adsorption. Based on molecular beam studies of $\mathrm{CO}$ adsorption on $\mathrm{Ni}(100)$ carried out by D'Evelyn et al. [32], the values of So and $\mathrm{K}$ were taken to be 0.9 and 0.25 , respectively.

The desorption probabilities of CO were calculated with the parameters $Q_{c o, n}=30 \mathrm{kcal} / \mathrm{mol}$ and $v=1015 \mathrm{~s}^{-1}$. These values are in the range of values reported in the literature for $\mathrm{CO}$ on $\mathrm{Ni}(100)$ [33. 36].

The simulation of C180 desorption in the absence of a C16O flux is displayed in fig. 7a. The principal feature of the spectrum is a peak at $430 \mathrm{~K}$ which is in good agreement with the location of the $\beta_{2}$ feature in the experimentally obtained spectrum shown in fig. 6a. At coverages greater than 0.50 , low-temperature features in the 300 $400 \mathrm{~K}$ range are present.

Simulations of the desorption of $\mathrm{C} 180$ in the presence of a flux of $\mathrm{C} 160$ are shown in fig. $7 \mathrm{~b}$. The peak at $430 \mathrm{~K}$ which is observed in the absence of the C160 flux is seen to decrease in intensity as the flux is increased. In addition, the C16O flux is seen to enhance the desorption of $\mathrm{C} 180$ at low temperature relative to the amount of C 180 observed at high temperature. The effect of the $\mathrm{C}_{160}$ flux on the C180 TPD spectra is qualitatively very similar to what is observed in the experimentally recorded spectra presented in fig. $6 \mathrm{~b}$.

The observation of enhanced desorption of $\mathrm{C}^{180}$ in the presence of a $\mathrm{C} 16 \mathrm{O}$ flux can be understood in the following way. The presence of gas-phase $\mathrm{C} 16 \mathrm{O}$ during the experiment leads to the adsorption of 
269
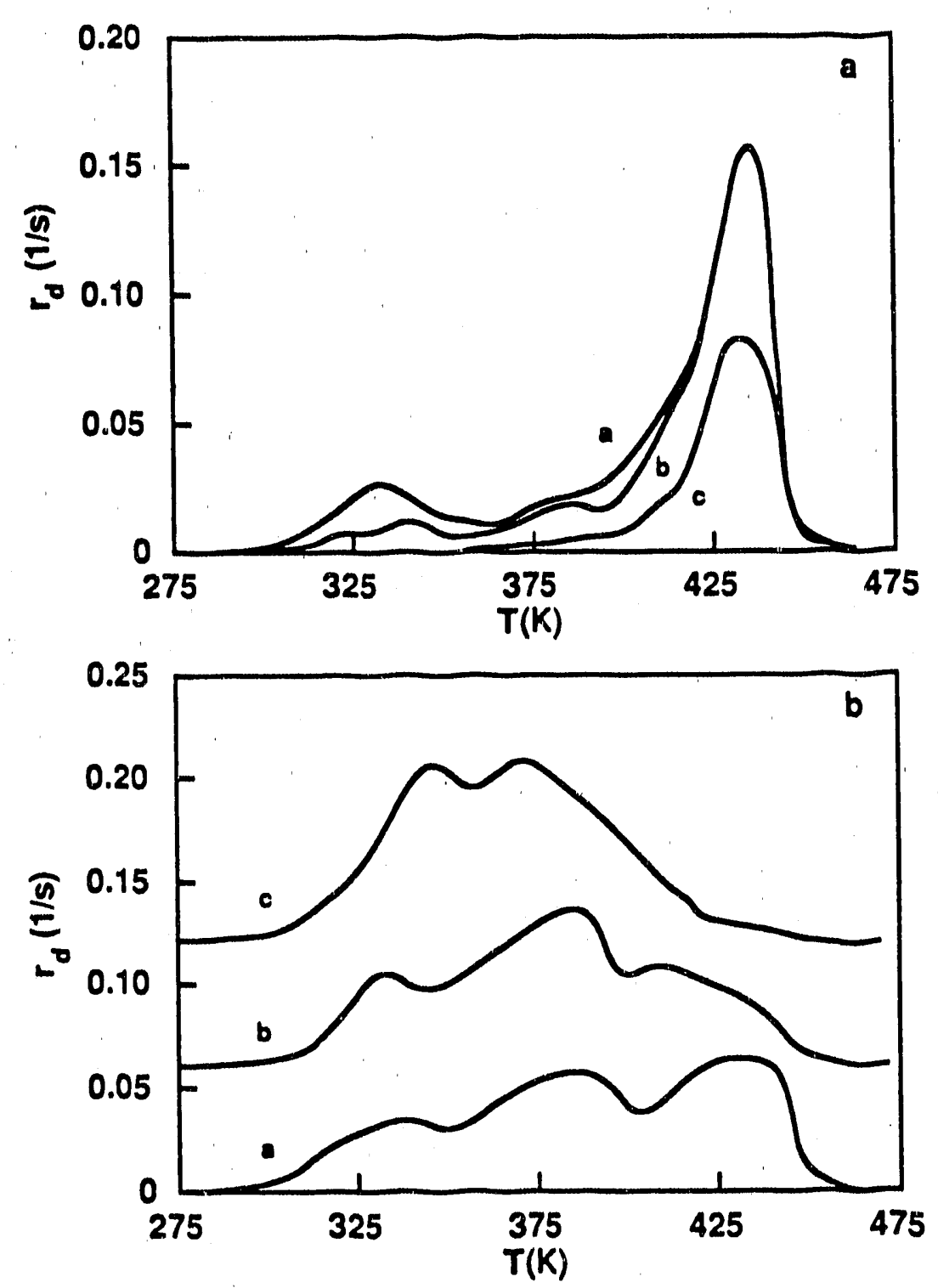

Fig. 7 Simulated TPD spectra of $\mathrm{C} 180$ desorption from $\mathrm{Ni}(100)$. a) Desorption in the absence of gas-phase $\mathrm{C} 160$. For curve $a, \theta^{\circ}\left(C^{180}\right)=0.60$; for curve $b, 0.55$; and for curve c, 0.25 ; b) Desorption of $C^{180}\left(\theta^{\circ}\left(C^{180}\right)=0.60\right)$ in the presence of gas-phase $C^{160}$. For curve $a$, the $C^{160}$ flux is $4.1 \times 1014 \mathrm{~cm}^{2} \mathrm{~s}^{-1}$; for curve b, $7.3 \times 1014 \mathrm{~cm}^{2} \mathrm{~s} \cdot 1$; and for curve c, $14.5 \times 1014 \mathrm{~cm}^{2} \mathrm{~s}^{-1}$. 
C160 and therefore to a larger value of $\theta_{T}$ at a given temperature. This conclusion is supported by fig. 8 a which shows the variation in $\theta_{T}$ as a function of temperature for different fluxes of $\mathrm{C} 160$. At low temperatures, the surface is saturated with $\mathrm{CO}$ independent of the magnitude of the $\mathrm{C} 160$ flux. At higher temperatures, the surface is no longer saturated and the total coverage is seen to increase with the intensity of the $\mathrm{C} 160$ flux.

The influence of coverage on the activation energy is illustrated in figs. $8 a$ and b. At low temperature, where the surface is saturated, both the coverage and activation energy are constant. In the temperature range between $350-450 \mathrm{~K}$, the total coverage increases with the flux, whereas the activation energy varies inversely with the coverage. The lowering of the activation energy with increased coverage is thus responsible for the enhanced rate of desorption at lower temperatures. This explanation is consistent with that offered by Yates and Goodman [4] to explain their experimental data.

\subsection{CONCLUSIONS}

A Monte Carlo simulation technique has been developed to represent the effects of adsorbate pressure on the rate of adsorbate desorption. The model accounts for the adsorption, surface diffusion, and desorption of adsorbates and for the effects of lateral interactions between adsorbed species. The influence of adsorbate coverage on the activation energy for desorption is described using the BOC-MP approach. Simulations of both isothermal and 


\section{1}
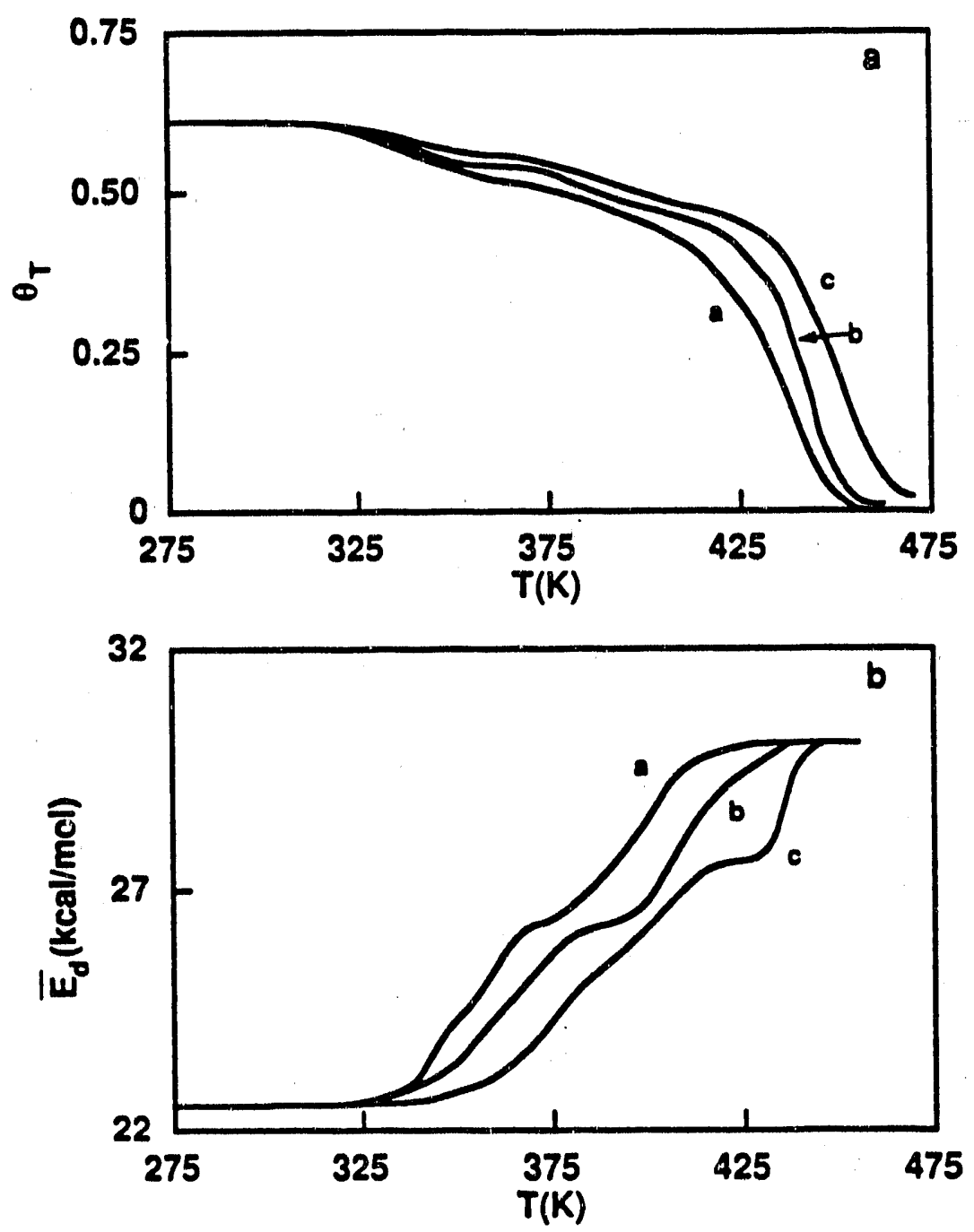

Fig. $8 \quad$ a) Variation in $\theta T$ versus $T$. b) Variation in the average $E_{d}$ for the desorbing $\mathrm{CO}$ molecules versus $\mathrm{T}$. In both panels, curve a corresponds to a $\mathrm{C} 160$ flux of $0 \mathrm{~cm}^{2} \mathrm{~s} \cdot 1$; curve $b$, $4.1 \times 1014 \mathrm{~cm}^{2} \mathrm{~s} \cdot 1$; and curve c, $14.5 \times 1014 \mathrm{~cm}^{2} \mathrm{~s} \cdot 1$. 


\section{2}

temperature-programmed desorption demonstrate that the rate of desorption observed in the presence of adsorbing gas-phase species is enhanced relative to that observed in the absence of gas-phase species. This behavior can be ascribed to repulsive lateral interactions between adsorbates which lower the activation energy for desorption. The Monte Carlo simulations also demenstrate that for a given adsorbate coverage, the activation energy for desorption of desorbing molecules can be significantly lower than that for all molecules present on the surface. This difference is a direct consequence of the nonuniform distribution of adsorbate molecules on the surface.

\section{ACKNOWLDGEMENTS}

This work was supported by the Director, Office of Basic Energy Sciences, Chemical Sciences Division of the US Department of Energy under contract DE-AC03-76SF00098. 


\section{APPENDIX}

The rationale for using the expressions for the probability of adsorption and desorption given by eqs. 1 and 3, respectively, is presented here. We show that these expressions can be derived from continuum rate expressions. The specific case considered is when the probabilities are normalized and all the environments on the surface are equivalent.

The rate of adsorption is given by

$$
\frac{d \theta}{d t}=F S(\theta)=k_{a} P S(\theta)
$$

where $k_{a}$ is the rate constant for adsorption and the other symbols have the same meaning as discussed earlier in this work. Equation A1 is a probability density function. Using standard relationships from probability theory, the probability of adsorption during a time interval $\Delta t$ is defined as:

$P^{\prime}=\Delta \theta=\int_{11}^{12} k_{a} P S(\theta) d t \approx k_{a} P S(\theta) \Delta t$

The number of adsorbates, $\mathrm{Na}$, which adsorb in the time interval $\Delta t$ is the product of $P^{\prime}$ and $G$, the sampling frequency of sites (i.e., the number of sites to which eq. A2 is applied). For a process which only depends only a single site, $G=N_{s}$, and $N_{a}$ is given as $N_{a}=P^{\prime} G=k_{a} P S(\theta) \Delta t N_{s}$

In order to incorporate eq. A3 into a Monte Carlo algorithm, it is convenient to factor eq. $\mathrm{A} 3$ into two terms, $\mathrm{Pa}$ and $\mathrm{Ga}$ which are given by:

$P^{a}=S_{0} k_{a} P \Delta t$ 
$G^{a}=\left[S(\theta) / S_{0}\right] N_{S}$

Equation $A 4$ is identical to eq. 1 and eq. $A 5$ is the expression used to determine the sampling frequency during the adsorption segment of an MCS.

Similar arguments apply to the process of desorption. The rate of non-associative desorption is given by

$-\frac{d \theta}{d t}=k_{d} \theta$

Equation $A 6$ can be used to define the probability of desorption:

$p^{\prime \prime}=|\Delta \theta|=\int_{11}^{12} k_{d} \theta d t=k_{d} \theta \Delta t$

The number of adsorbates which desorb in the time interval $\Delta t$ is

$N_{d}=P^{\prime \prime} G=k_{d} \theta \Delta t N_{s}$

Equation $A 8$ can be factored into two terms, $P d$ and $G d$, which are given by:

$P^{d}=k_{d} \Delta t$

$G^{d}=\theta N_{S}$

Equation A9 is identical to eq. 3 and eq. $A 10$ is the expression used to determine the sampling frequency during the desorption segment of an MCS.

The derivations presented above establish the connection between the more familiar continuum rate expressions (eqs. $A 1$ and A6) and the Monte Carlo probability expressions (eqs. A4 and A9). The accuracy of the approximation $d t=\Delta t$ in eqs. $A 2$ and $A 7$ depends on the value of the step size $\Delta t$. As a rule of thumb, $\Delta t$ was chosen so that the maximum number of adsorbates which adsorb or desorb 
in a segment of an MCS was less than ten percent of the saturation coverage. As seen in fig. 5 , specifying $\Delta t$ in this fashion yields results which are in reasonable agreement with the numerical solution of the differential equations. The accuracy of the Monie Carlo approach has also been discussed in ref. [21] for the case of first- and second-order desorption kinetics. 
References

[1] K. Klier, A.C. Zettlemoyer and H. Leidheiser, Jr., J. Chem. Phys. (1970) 589.

[2] P.W. Tamm and L.D. Schmidt, J. Chem. Phys. 52 (1970) 1150.

[3] T. Matsushima, J. Catal. 64 (1980) 38.

[4] J.T. Yates, Jr. and D.W. Goodman, J. Chem. Phys. 73 (1980) 5371.

[5] T. Yamada, T. Onishi and K. Tamaru, Surf. Sci. 133 (1983) 533.

[6] T. Yamada, T. Onishi and K. Tamaru, Surf. Sci. 157 (1985) L389.

[7] T. Yamada and K. Tamaru, Surf. Sci. 139 (1984) 463.

[8] T. Yamada and K. Tamaru, Surf. Sci. 138 (1984) L155.

[9] T. Yamada and K. Tamaru, Surf. Sci. 146 (1984) 341.

[10] T. Yamada, Z. Runsheng, Y. Iwasawa and K. Tamaru, Surf. Sci. 205 (1988) 82.

[11] K. Tamaru, in: Springer Scries in Surface Sciences, Vol. 14, ed. F.W. DeWette (Springer-Verlag, Berlin 1988) 114.

[12] K. Tamaru, T. Yamada, Z. Runsheng and Y. Iwasawa, in: Proc. 9th Intern. Congress Catal., Calgary, (1988) 1006.

[13] K. Tamaru, in: Studies in Surface Science and Catalysis, Vol. 54, eds. M. Misona, Y. Moro-oka and S. Kimura (Elsevier, Amsterdam 1990) 32.

[14] J.D. Beckerle, A.D. Johnson, Q.Y. Yang and S.T. Ceyer, in: Springer Series in Surface Sciences, Vol. 14, ed. F.W. DeWette (SpringerVerlag, Berlin 1988) 109.

[15] S.T. Ceyer, discussion in: Springer Series in Surface Sciences, Vol. 14, ed. F.W. DeWette (Springer-Verlag, Berlin 1988) 118.

[16] J.D. Beckerle, A.D. Johnson, Q.Y. Yang and S.T. Ceyer, J. Vac. Sci. Technol. A 6 (1988) 903.

[17] J.D. Beckerle, A.D. Johnson and S.T. Ceyer, Phys. Rev. Lett. 62 (1989) 685.

[18] K. Binder, Ed., Monte Carlo Methods in Statistical Physics (Springer, Berlin, 1979).

[19] E. Shustorovich, Surf. Sci. Rept. 6 (1986) 1.

[20] E. Shustorovich, in: Advances in Catalysis, Vol. 37, eds. D.D. Eley, H. Pines and P.B. Weisz (Academic Press, New York) in press.

[21] S.J. Lombardo and A.T. Bell, Surf. Sci. 206 (1988) 101.

[22] S.J. Lombardo and A.T. Bell, Surf. Sci. 224 (1989) 451.

[23] IMSL, Subroutine GGUBFS, Houston, TX 1980. 
[24] J.C. Tracy and P.W. Palmberg, J. Chem. Phys. 51 (1969) 4852.

[25] P. Kisliuk, J. Phys. Chem. Solids 3 (1957) 95; 5 (1958) 78.

[26] R.J. Behm, K. Christmann, G. Ertl, and M.A. Van Hove, J. Chem. Phys. 73 (1980) 2984.

[27] A.M. Bradshaw and F.M. Hoffmann, Surf. Sci. 72 (1978) 513.

[28] G.M. Bliznakov and M.P. Kiskinova, J. of Catal. 61 (1980) 305.

[29] H. Conrad, G. Ertl; J. Koch, and E.E. Latta, Surf. Sci. 43 (1974) 462.

[30] V.P. Zhdanov, Surf. Sci. 157 (1985) L384.

[31] G.E. Mitchell, J.L. Gland and J.M. White, Surf. Sci. 131 (1983) 167.

[32] M.P. D'Evelyn, H.-P. Steinrück and R.J. Madix, Surf. Sci. 180 (1987).

[33] J.C. Tracy, J. Chem. Phys. 56 (1972) 2736.

[34] K. Christmann, O. Schober, and G. Ertl, J. Chem. Phys. 60 (1974). 4719.

[35] J.B. Benziger and R.J. Madix, Surf. Sci. (1979) 394.

[36] B.E. Koel, D.E. Peebles and J.M. White, Surf. Sci. 125 (1983) 709. 


\section{Appendix}

FORTRAN Programs for Simulating TemperatureProgrammed and Isothermal Desorption Kinetics

A description of the FORTRAN programs and subroputines used to perform the calculations described in this work is presented here. MONCAR70 is a main calling program used to simulate temperatureprogrammed desorption of two adsorbates coadsorbed on a surface. MONCAR15 is a main calling program used to simulate isothermal desorption in the presence of an adsorbing species. The listing of these two programs is provided at the end of the Appendix. 


\section{Description of MONCAR7O}

MONCAR70 is a calling program, written in FORTRAN, for simulating TPD spectra of one or two species, named $A$ for adsorbate and $C$ for coadsorbate, adsorbed on either an fcc or bcc (100) surface. The adsorbates $A$ and $C$ can occupy on-top, bridge, or hollow sites. The adsorbates must, however, be restricted to one type of site, and they both cannot have the same type of coordination (i.e., both cannot be bridged bonded). All parameters which are needed to perform a s.mulation are contained in MONCAR70. Variable initialization, overall program flow, and data output are all controlled in this program.

The surface array used in the simulations is a 100 by 100 logical array of numbered sites and all sites (on-top, bridge, and hollow) are numbered sequentially with the indices $(1, \mathrm{~J})$. If a site is occupied, it has a value "TRUE"; if unoccupied, it has a value of "FALSE". Site numbering begins in the upper left corner of the array with the on-top site numbered $(1,1)$. A schematic of the site numbering is shown in fig. 1. Examples of on-top locations are $(1,1)$, $(3,5)$, and $(99,99)$; examples of bridge locations are $(1,2),(2,99)$, and $(99,100)$; and examples of hollow locations are $(2,2),(2,4)$, and $(98,98)$. The location of an $(1, J)$ pair of coordinates can be determined by the following set of rules applied to the array indices $(I, J)$ :

For on-top sites, the sum $I+J$ is even and $I$ is odd;

For bridge sites, the sum $1+J$ is odd;

For hollow sites, the sum $1+J$ is even and $I$ is even.

In order to eliminate edge effects, periodic boundary conditions are used. In addition, the following corner or near-corner sites are never allowed to be occupied: $(1,1),(1,2),(2,1),(2,2),(1,99),(2,99)$, $(1,100),(2,100),(99,1),(99,2) .(100,1),(100,2),(99,99),(99,100)$, $(100,99)$, and $(100,100)$. These 16 sites, which represent a small fraction of the toial number of sites, are kept unoccupied because it is computationally expensive to account for them in simulations. 

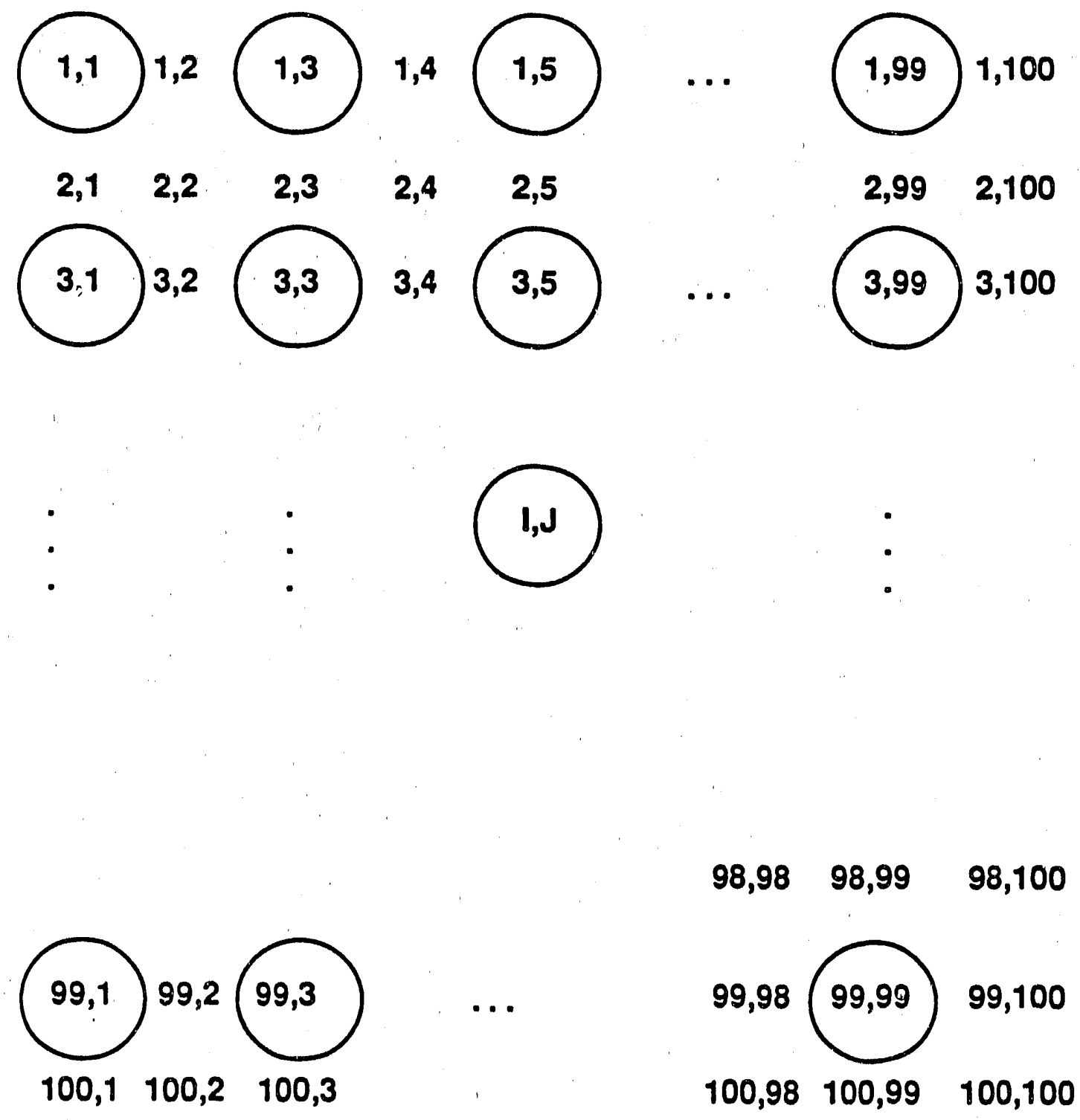

Fig. 1 Surface map showing the numbering scheme $(1, J)$ of the on-top, bridge, and hollow sites. The open circles are the metal atoms. 
To perform a simulation, MONCAR70 calls a number of subroutines. The overall hierarchy of MONCAR70 is as follows:

1) Variables are defined and initialized.

2) The initial coverages are placed on the surface arrays by calling subroutines INTTCP,INTBRI, and INTHOL.

3) The temperature for the Monte Carlo step (MCS) is initialized.

The desorption segment of the MCS is conducted by calling subroutines DESORT,DESORB, and DESORH.

4) The diffusion segment of the MCS is conducted by calling subroutines DIFTOP, DIFBRI, and DIFHOL.

5) The output variables for the MCS are written to a data file. The temperature is increased and steps 3,4 , and 5 are repeated a specified number of times.

6) The simulation ends.

Each subroutine called by MONCAR70 is highly modular in nature and performs a specific function. The subroutines and their arguments are listed below along, with a brief description. In general, names of subroutines, arguments, or variables having to do with on-top sites or species have. "T" or "TOP" in their name. In a similar fashion, names which apply to bridge sites or species have "B" or "BRI" in their name and names which apply to hollow sites or species have "H" or "HOL".

Subroutines (Arguments)

INTTOP(COVTI,LOCT)

Initializes the coverage in the on-top sites. COVTI is the value of the coverage to be initialized and LOCT indicates whether the coverage is to be random or ordered.

INTBRI(COVBI,LOCB)

Initializes the coverage in the bridge sites. COVBI is the value of the coverage to be initialized and LOCB indicates whether the coverage is to be random or ordered.

INTHOL(COVHI,LOCH)

Initializes the coverage in the hollow sites. COVHI is the value of the coverage to be initialized and $\mathrm{LOCH}$ indicates 
whether the coverage is to be random or ordered.

DESORT(SAMTOP,COUNTT,NSVIST,BET,AET,BETD,AETD)

Treats the non-associative desorption of adsorbates from ontop sites. SAMTOP is the sampling frequency of occupied ontop sites during an MCS. COUNTT is a running count of the number of on-top species which desorb in an MCS. NSVIST is a running count of the number of successful visits to on-top sites (i.e., occupied sites) during an MCS. BET is a running sum of the binding energies for all on-top species visited during an MCS. BETD is a running sum of the binding energies for all ontop species which desorb during MCS. AET is a running sum of activation energies of desorption for all on-top species visited during an MCS. AETD is a running sum of the activation energies of desorption for the on-top species desorbing during an MCS.

\section{DESORB(SAMBRI,COUNTB,NSVISB,BEB,AEB,BEBD,AEBD)}

Treats the non-associative and associative desorption of adsorbates from bridge sites. The arguments are similar to those described for DESORT.

DESORH(SAMHOL,COUNTH,NSVISH,BEH,AEH,BEHD,AEHD)

Treats the non-associative and associative desorption of adsorbates from hollow sites. The arguments are similar to those described for DESORT.

\section{DIFTOP(NJMPT)}

Treats jumps of adsorbates between on-top sites. NJMPT is a running count of the number of jumps between on-top sites during an MCS.

\section{DIFBRI(NJMPB)}

Treats jumps of adsorbates between bridge sites. NJMPB is a running count of the number of jumps between bridge sites during an MCS.

DIFHOL(NJMPH)

Treats jumps of adsorbates between hollow sites. NJMPH is a 
running count of the number of jumps between hollow sites during an MCS.

QONTOP $(1, J, Q T)$

Calculates the binding energy $Q T$ for an on-top site $(I, J)$ with the BOC-MP method.

QBRIDG(I,J,QB)

Calculates tine binding energy $Q B$ for a bridge site $(I, J)$ with the BOC-MP method.

QHOLLO(I,J,QH)

Calculates the binding energy $\mathrm{QH}$ for an fcc(100) hollow site $(I, J)$ with the BOC-MP method.

$\mathrm{QBCCHO}(\mathrm{I}, \mathrm{J}, \mathrm{QH})$

Calculates the binding energy $\mathrm{QH}$ for a bcc(100) hollow site with the BOC-MP method.

LOCTT $(1, J, I T T, J T T)$

Given an on-top $(I, J)$ location, returns arrays ITT and JTT of the four nearest-neighbor on-top locations.

LOCTB(I,J,ITB,JTB)

Given an on-top $(I, J)$ location, returns arrays ITB and JTB of the four nearest-neighbor bridge locations.

L.OCTH(I,J,ITH,JTH)

Given an on-top $(I, J)$ location, returns arrays ITH and JTH of the two nearest-neighbor hollow locations.

LOCBT(I,J,IBT,JBT)

Given a bridge $(I, J)$ location, returns arrays IBT and JBT of the four nearest-neighbor on-top locations.

LOCBB(I,J,IBB,JBB)

Given a bridge $(1, J)$ location, returns arrays $I B B$ and JBB of the four nearest-neighbor bridge locations. 
$\mathrm{LOCBH}(\mathrm{I}, \mathrm{J}, \mathrm{IBH}, \mathrm{JBH})$

Given a bridge $(1, J)$ location, returns arrays $\mathrm{IBH}$ and $\mathrm{JBH}$ of the two nearest-neighbor hollow locations.

LOCBBN(I,J,IBBN,JBBN)

Given a bridge $(I, J)$ location, returns arrays IBBN and JBBN of the four next-nearest-neighbor bridge locations.

$\operatorname{LOCHT}(1, J, I H T, J H T)$

Given a hollow $(I, J)$ location, returns arrays $I H T$ and $J H T$ of the four nearest-neighbor on-top locations.

$\operatorname{LOCHB}(I, J, I H B, J H B)$

Given a hollow $(I, J)$ location, returns arrays $\mathrm{HHB}$ and JHB of the four nearest-neighbor bridge locations.

$\mathrm{LOCHH}(\mathrm{I}, \mathrm{J}, \mathrm{IHH}, \mathrm{JHH})$

Given a holiow $(1, J)$ location, returns arrays $\mathrm{IHH}$ and $\mathrm{JHH}$ of the four nearest-neighbor hollow locations.

LOCHHN(I,J,IHHN,JHHN)

Given a hollow $(I, J)$ location, returns arrays IHHN and JHHN of the four next-nearest-neighbor hollow locations.

SURMAP

Creates a TELAGRAF plotting file of the positions of the surface atoms and the adsorbates.

A listing of the important variables and arrays in MONCAR70 and their meanings are given next. Names which have already been mentioned above as subroutine arguments are not described again.

The convention for using "T"or "TOP" to relate to on-top, "B" or "BRI" to relate to bridge, and " $\mathrm{H}^{n}$ or "HOL" to relate to hollow, still applies.

Name

$T(100,100)$

$B(100,100)$

$H(100,100)$
Meaning

Surface array for the occupancy of on-top sites. Surface rray for the occupancy of bridge sites. Surface array for the occupancy of hollow sites. 


\section{5}

DSEED

$R$

SITES

TEMP

TMIN

BETA

DELT

KDO

DELKDO

TOP

BRI

$\mathrm{HOL}$

$B C C$

MODT

MODB

$\mathrm{MOOH}$

QOT

COB

$\mathrm{QOH}$

TT

TB

TH

BB
Seed variable for the random number generator.

Gas constant.

Number of surface metal atoms or sites.

Temperature of the surface (K).

Initial temperature.

Heating rate $(\mathrm{K} / \mathrm{s})$.

Time interval (s).

Preexponential factor for desorption (1/s).

The product of DELT and KDO.

Denotes if on-top sites sites are used (1) or not (0).

Denotes if bridge sites sites are used (1) or not (0). Denotes if hollow sites sites are used (1) or not (0).

Denotes if the hollow site is a bcc(100) hollow site (1) or a fcc(100) hollow site (0).

Denotes the mechanism of desorption for species in on-top sites: (1) for non-associative and (2)for associative desorption.

Denotes the mechanism of desorption for species in bridge sites: (1) for non-associative and (2)for associative desorption.

Denotes the mechanism of desorption for species in hollow sites: (1) for non-associative and (2)for associative desorption.

Heat of adsorption in the on-top position for the ontop species.

Heat of adsorption in the on-top position for the bridge species.

Heat of adsorption in the on-top position for the hollow species.

Denotes whether direct on-top to on-top adsorbate interactions occur (1) or not (0).

Denotes whether direct on-top to bridge adsorbate interactions occur (1) or not (0).

Denotes whether direct on-top to hollow adsorbate interactions occur (1) or not (0).

Denotes whether direct bridge to bridge adsorbate interactions occur (1) or not (0). 
$\mathrm{BH}$

$\mathrm{HH}$

BOTT

BOTB

BOTH

BOBB

BOBH

$\mathrm{BOH}$

DETT

DETB

DETH

DEBB

DEBH

DEH

SURPLO

IM

$I P(1)$

COVDES(1)

GCOUNT

GOOUNB
Denotes whether direct bridge to hollow adsorbate interactions occur (1) or not (0).

Denotes whether direct hollow to hollow adsorbate interactions occur ( 1 ) or not (0).

Bond order for on-top to on-top direct adsorbate interactions.

Bond order for on-top to bridge direct adsorbate interactions.

Bond order for on-top to hollow direct adsorbate interactions.

Bond order for bridge to bridge direct adsorbate interactions.

Bond order for bridge to hollow direct adsorbate interactions.

Bond order for hollow to hollow direct adsorbate interactions.

Dissociation energy for on-top to on-top direct adsorbate interactions.

Dissociation energy for on-top to bridge direct adsorbate interactions.

Dissociation energy for on-top to hollow direct adsorbate interactions.

Dissociation energy for bridge to bridge direct adsorbate interactions.

Dissociation energy for bridge to hollow direct adsorbate interactions.

Dissociation energy for hollow to hollow direct adsorbate interactions.

Denotes whether surface maps are (1) or are not (0) to be made.

Switch variable to limit the printirig of the coordinates of metal atoms to once.

Switch variable to limit the printing of adsorbate coordinates for a specified coverage to once. Array containing the coverage at which a surface map is to be made.

Global (total) - running count of the number of species which have desorbed from on-top sites: Global (total) running count of the number of 
species which have desorbed from bridge sites.

GOONH

COVT

COVB

$\mathrm{COVH}$

RATET

RATEB

RATEH
Global (total) running count of the number of species which have desorbed from hollow sites.

Running value of the coverage in the on-top sites. Running value of the coverage in the bridge sites. Running value of the coverage in the hollow sites. Rate of desorption for species in on-top sites for an MCS.

Rate of desorption for species in bridge sites for an MCS.

Rate of desorption for species in hollow sites for an MCS. 


\section{Description of MONCAR15}

MONCAR15 is a calling program, written in FORTRAN, for simulating isothermal adsorption, diffusion, and desorption of one or two species, named $A$ (for adsorbate) and $C$ (for coadsorbate), adsorbed on an $f c c(100)$ surface. Species $A$ and $C$ have identical energy parameters (they are isotopes) and they both occupy bridge sites. All parameters which are needed to perform a simulation are contained in MONCAR15. Variable initialization, overall program flow, and data output are all controlled in this program. MONCAR15, and the subroutines called by it, are very similar in structure to MONCAR70 and the subroutines described earlier. The surface array (see fig. 1) is identical to that described earlier.

In order to perform a simulation, MONCAR15 calls a number of subroutines. The overall hierarchy of MONCAR15 is as follows:

1) Variables are defined and initialized.

2) The initial coverages are placed on the surface arrays by calling subroutine INTBTW.

3) The adsorption segment of a Monte Carlo step (MCS) is conducted by cailing subroutine ADSBRI.

4) The diffusion segment of an MCS is conducted by calling subroutine DIFBTW.

5) The desorption segment of an MCS is conducted by calling subroutine DESBTW.

6) The output variables for an MCS are written to a data file. The time is incremented and steps $3,4,5$, and 6 are repeated a specified number of times.

6) The simulation ends.

The subroutines mentioned above and their arguments are listed below along with a brief description. Names of subroutines, arguments, or variables having to do with bridge sites or species have "B" or "BRI" in their name, and, in addition, an " $A$ " or " $C$ " is added to the names to differentiate between species $A$ or $C$. 
Subroutines (Arguments)

INTBTW(COVBAI,LOCBA,COVBCI,LOCBC)

Initializes the coverage in the bridge sites. COVBAI and COVBCl are the values of the coverages of $A$ and $C$ to be initialized, respectively. $\angle O C B A$ and $\angle O C B C$ indicates whether the coverages of $A$ and $C$ are to be random or ordered.

\section{ADSBRI(SAMFRB,CTADC)}

Treats the non-dissociative adsorption of species C. SAMFRB is the sampling frequency of unoccupied bridge sites. CTADC is a running count of the number of $C$ species adsorbed.

DIFBTW(NJMP2)

Treats jumps of adsorbates between bridge sites. NJMP2 is a running count of the number of jumps between bridge sites during an MCS.

\section{DESBTW(SAMFDA,SAMFDC,COUNTA,COUNTC,AEAD,AECD,AEA,AEC, NSVISA,NSVISC)}

Treats the non-associative desorption of adsorbates $A$ and $C$ from bridge sites. SAMFDA and SAMFDC are the sampling frequencies of occupied bridge sites for $A$ and $C$ during an MCS. COUNTA and COUNTC are running counts of the number of bridge $A$ and $C$ species which desorb in a MCS. AEAD and AEAC are running sums of the activation energies for all bridge $A$ and $C$ species visited during an MCS. AEA and AEC are running sums of activation energies of desorption for all bridge $A$ and $C$ species visited during an MCS. NSVISA and NSVISC is a running count of the number of successful visits to bridge $A$ and $C$ sites (i.e., occupied sites) during an MCS.

The calling programs MONCAR70 and MONCAR15 are listed on the next pages. 


\section{Ma1n: MOXCAR70}

C TWO TYPES OF ADSORBATES CAN BE ACCOMODATED ON A FOUR-FOLD
C ROTATIONAL SURFACE. THE THREE SURFACE ARRAYS ARE T $(100,100)$, C $B(100,100)$ AND $H(100,100)$. SITE DIFFERENTIATION IS BY

MONOO410

MONO0420

MONOO430

MONO0440

MONOO45O

MONOO460

MONOO470

MONO0480

MONOO49O

MONO0500

MONO0510

MONOO520

MONOO530

MONOO54O

MONOO550

MONO0 660

MONOO570

MONO0580

MONO0590

MONO0600

MONO0610

MONO0620

MONO0630

MONO0640

MONO0650

MONO0 660

MONO0670

MONO0680 


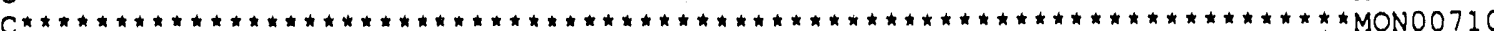

INITIALIZE COVERAGE EITHER RANDOM(I) OR ORDERED(0) BY LOCI.

MONO0720

DATA COVTI, LOCT, COVBI, LOCB, COVHI, IOCH/0.5D0,1,2.0D0,0,1D0, 01

MONO0730

IF (TOP.EQ.1) THEN ENDIF

CALL INTTOP (COVTI, LOCT)

IF (BRI.EQ.1) THEN

CALI INTBRI (COVBI, LOCB)

ENDIF

IF (HOL.EQ.1) THEN ENDIF

CALL INTHOL (COVHI, LOCH)

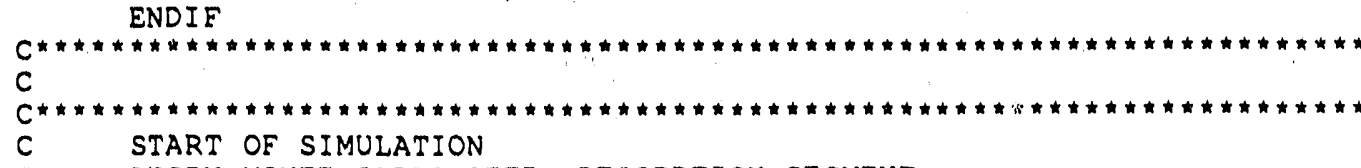

MONO0740

MONO0750

MON00760

MONO0770

MONO0780

MONO0790

MON 10800

MONOOOB10

MONO0820

MONO0830

MONO0840

START OF SIMULATION

C BEGIN MONTE CARLO STEP: DESORPTION SEGMENT

DO $100 \quad N M=1,133$

TEMP $=T E M P+B E T A * D E L T$

COVT $=($ (COVTI SITES $)-$ GCOUNT $) /$ SITES

COVB $=(($ COVBI *SITES $)-$ GCOUNB $) /$ SITES

COVH $=($ (COVHI *SITES)-GCOUNH $) /$ SITES

$\operatorname{COV}(1)-\operatorname{COVB}$

$\operatorname{COV}(2)=\operatorname{COV} B$

$O V(3)=C O V B$

IF (SURPLO.EQ.1) THEN

ENDIF

CAIL SURMAP (COVT, COVB, COVH, COV, COVDES, IP, IM)

COUNTT $=0$

COUNTB $=0$

COUNTH $=0$

NSVIST $=0$

NSVI $S B=0$

NSVI SH $=0$

$A E T=0$

$A E B=0$

$A E H=0$

$B E T=0$

$B E B=0$

$B E H=0$

$A E T D=0$

$A E B D=0$

$A E H D=0$

$B E T D=0$

$B E B D=0$

$B E H D=0$

NJMPT $=0$

$N J M P B=0$

NJMPH $=0$

IF (MODT.EQ.1) THEN

ELSE'

SAMTOP $=$ SITES *COVT

ENDIE

SAMTOP=SITES* (COVT**2)*.5DO

IF (MODB.EQ.1) THEN

EISE

SAMBR I - SITES * COVB

ENDIF

SAMBRI = SITES* $(C O V B * 2) *$. SDO

IF (MODH.EQ.1) THEN

ELSE SAMHOL=SITES * COVH ENDIF

SAMHOL-SITES " (COVH* *2)*.5DO

MONOD860

MONO0870

MONO0880

MON00890

MONO0900

MONO0910

MONOO920

MONO0930

MONOO940

MONOO950

MONO0960

MONO0970

MONO0980

MONOO990

MONO100U

MONO1010

MONO1020

MONO1030

MONO1040

MONO1050

MONO1060

MONO1070

MONO1080

MONO1090

MONO1100

MONO1110

MONO 1120

MONO1130

MONO1140

MONO1150

MONO1160

MONO1170

MONO1180

MONO1190

MONO1200

MONO1210

MONO1220

MONO1230

MONO1240

MONO1250

MONO1260

MONO1270

MONO1280

MONO1290

MONO1300

MONO1310

MONO1320

MONO1330

MONO1 340

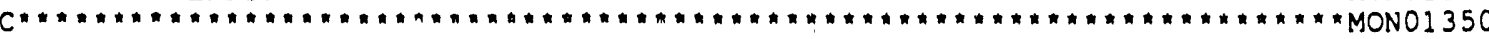

C DETERMINE DESORPTION OF SPECIES.

MONO1360

DO $55 \mathrm{~N}=1,5000$

C CALL DESORT (SAMTOP, COUNTT, NSVIST, BET, AET, BETD, AETD)

CALL DESORB (SAMBRI, COUNTB, NSVISB, BEB, AEB, BEBD, AEBD)

C

CALL DESORH (SAMHOL, COUNTH, NSVISH, BEH, AEH, BEHD, AEHD)

MONO1370

MONO1380

MONO1390

MONO1400 


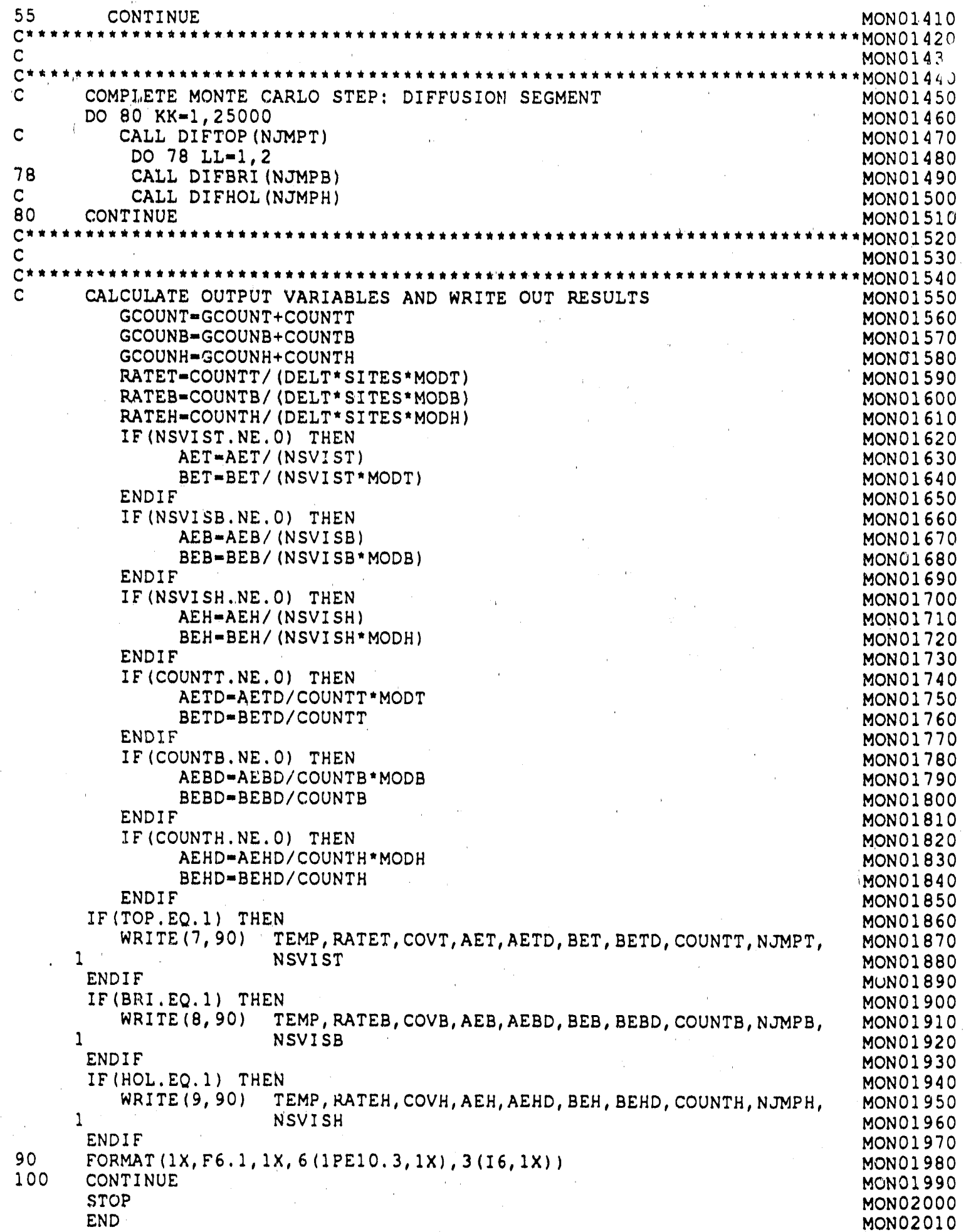




\section{Maln: MOBCAR15}

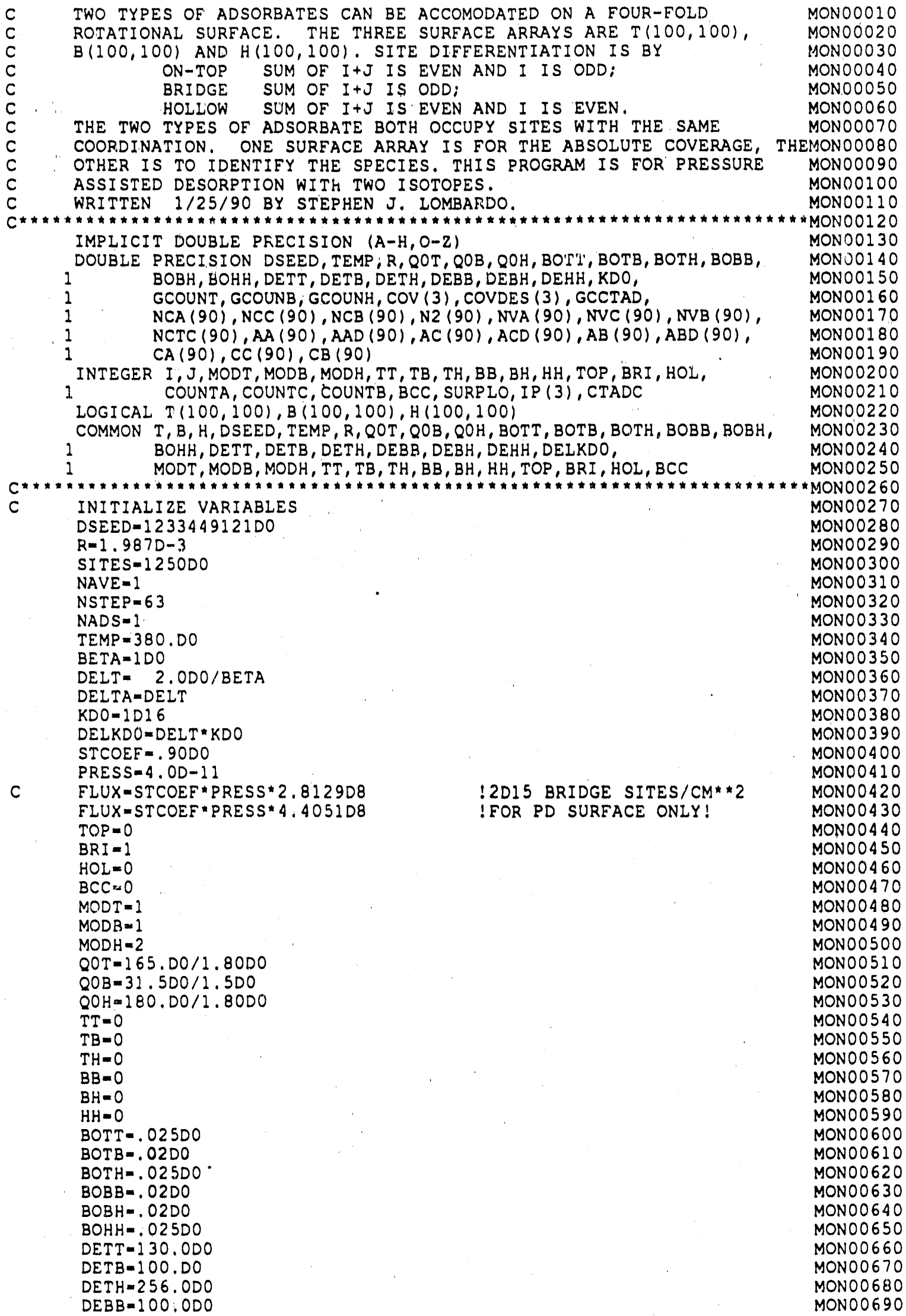




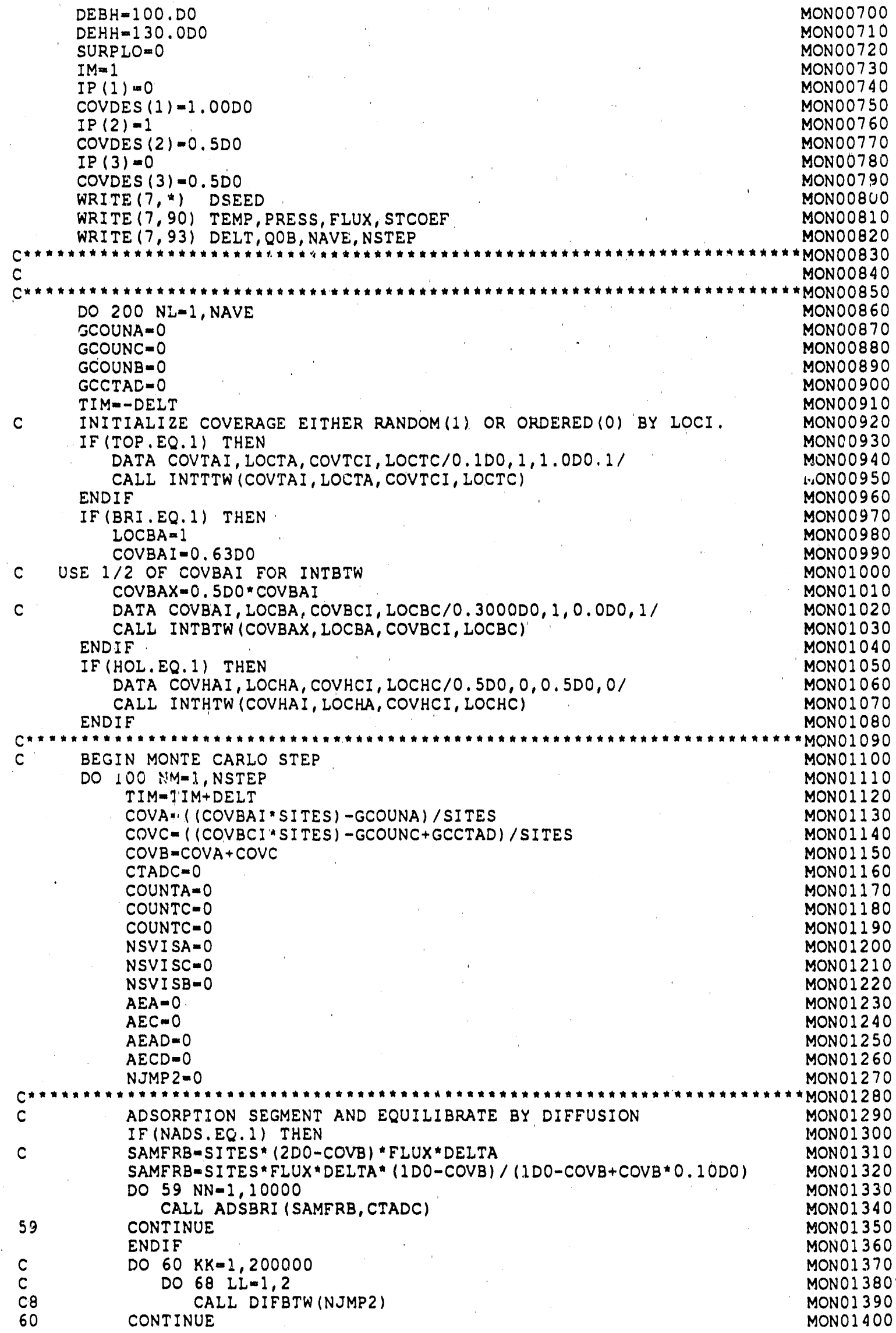




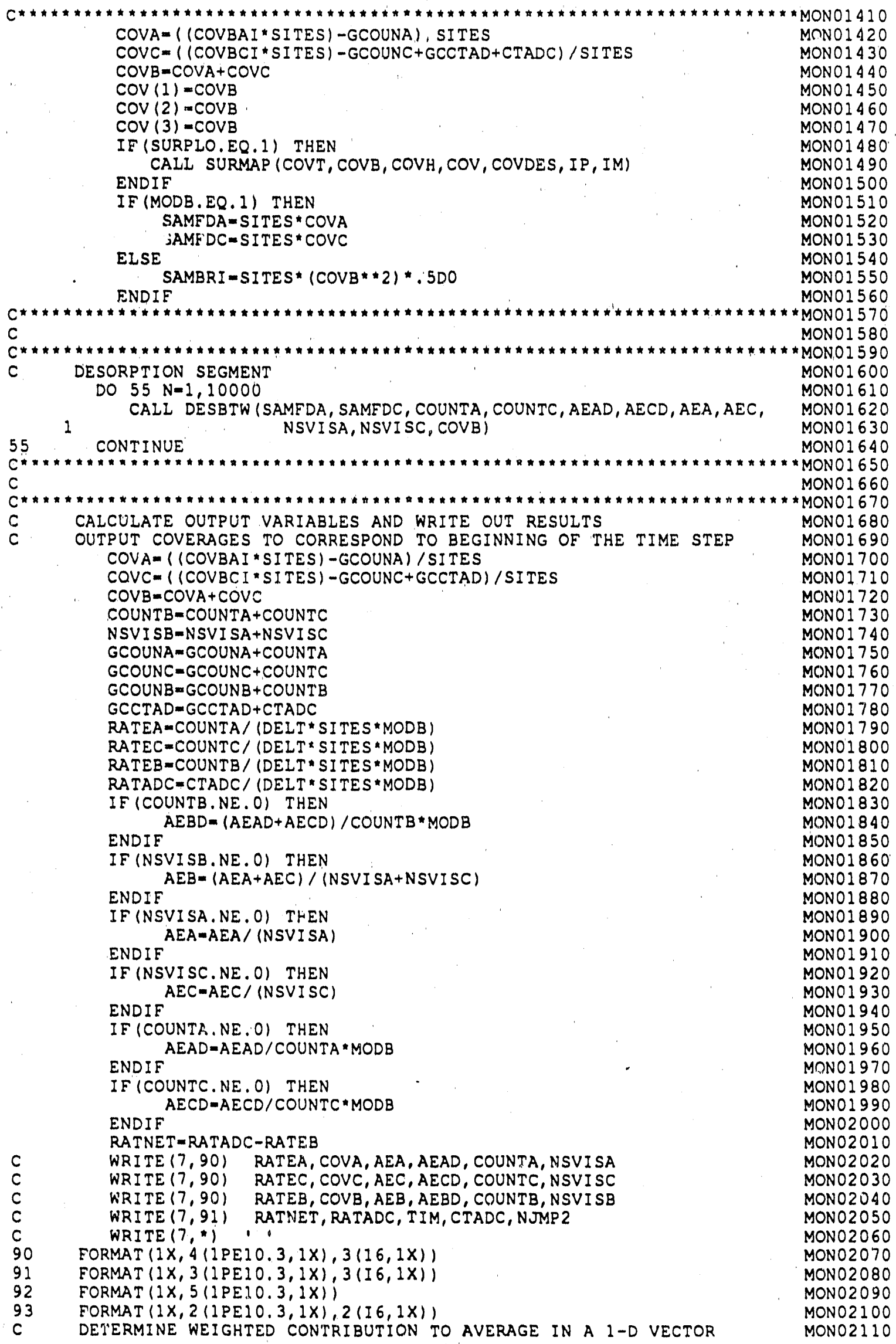


$\mathrm{N} 2(\mathrm{NM})=\mathrm{NJMP} 2+\mathrm{N} 2(\mathrm{NM})$

MONO2120

$C A(N M)=C O V A$ * SITES+CA (NM)

$A A(N M)=A E A * N S V I S A+A A$ (NM)

$A A D(N M)=A E A D * C O U N T A+A A D(N M)$

$N C A(N M)=C O U N T A+N C A(N M)$

NVA (NM) $=$ NSVI SA $+N V A$ (NM)

$C C(N M)=C O V C \star S I T E S+C C(N M)$

$A C(N M)=A E C * N S V I S C+A C$ (NM)

$A C D(N M)=A E C D * C O U N T C+A C D(N M)$

NCC (NM) $=$ COUNTC+NCC (NM)

NVC (NM) $=$ NSVI SC+NVC (NM)

$C B(N M)=C O V B \star S I T E S+C B(N M)$

$A B(N M)=A E B * N S V I S B+A B(N M)$

$A B D(N M)=A E B D * C O U N T B+A B D(N M)$

$\mathrm{NCB}(\mathrm{NM})=\mathrm{COUNTB}+\mathrm{NCB}(\mathrm{NM})$

$\mathrm{NVB}(\mathrm{NM})=\mathrm{NSVI} S \mathrm{~S}+\mathrm{NVB}(\mathrm{NM})$

$\operatorname{NCTC}(N M)=C T A D C+N C T C(N M)$

100 CONTINUE

200 CONTINUE

DETERMINE AVERAGES

WRITE $(7, *)$ 'AVERAGES'

$T I M=-D E L T$

DO $300 \mathrm{NZ}=1$, NSTEP

$T I M=T I M+D E L T$

DAVE $=D B L E$ (NAVE)

$X R A=N C A(N Z) /(D E L T * S I T E S * M O D B * D A V E)$

$X C A=C A(N Z) /(S I T E S * D A V E)$

IF (NVA (NZ). EO.ODO) THEN

$X A A=O D O$

ELSE

$X A A=A A(N Z) /(N V A(N Z))$

ENDIF

IF (NCA (N2) .EO.ODO) THEN

XAAD $=0 D O$

ELSE

$X A A D=A A D(N Z) /(N C A(N Z))$

ENDIF

$X N C A-N C A$ (NZ) /DAVE

$X R C=N C C(N 2) /(D E L T * S I T E S * M O D B * D A V E)$

$X C C=C C(N Z) /(S I T E S * D A V E)$

IF (NVC (NZ).EQ.ODO) THEN

ELSE $X A C=O D O$

ENDIF

$A C=A C(N Z) /(N \vee C(N Z))$

IF (NCC (NZ).EQ.ODO) THEN

$X A C D=O D O$

ELSE

$X A C D=A C D(N Z) /(N C C(N Z))$

ENDIF

$X N C C=N C C(N Z) / D A V E$

$X R B=N C B(N 2) /(D E L T$ * SITES *MODB *DAVE)

$X C B=C B(N Z) /(S I T E S * D A V E)$

IF (NVB (NZ) . EO.ODO) THEN

$$
X A B=O D O
$$

ELSE

$X A B=A B(N Z) /(N V B(N Z))$

ENDIF

IF (NCB (NZ).EQ.ODO) THEN

$X A B D=O D O$

ELSE

$X A B D=A B D(N Z) /(N C B(N Z))$

ENDIF

$X N C B=N C B(N 2) / D A V E$

$X N C T C=N C T C(N Z) / D A V E$

$X R A D C=N C T C(N Z) /(D E L T A * S I T E S$ * (1ODB*DAVE)

$X R N E T-X R A D C-X R B$

$X N 2=N 2$ (N2) / DAVE

WRITE $(7,92)$ XRA, XCA, XAA, XAAD, XNCA

WRITE $(7,92) \quad X R C, X C C, X A C, X A C D, X N C C$

WRITE $(7,92) \quad X R B, X C B, X A B, X A B D, X N C B$

MONO2130

MONO2140

MONO2.150

MONO2160

MONO2170

MONO2180

MONO2190

MONO2200

MONO2210

MONO2220

MONO2230

MONO2240

MONO2250

MONO2260

MONO2270

MONO2280

MONO2290

MONO2300

MONO2310

MONO2320

MONO2330

MONO2340

MON02350

MONO2360

MONO2370

MONO2 380

MONO2390

MONO2400

MONO2410

MONO2420

MONO2430

MONO2440

MONO2450

MONO2460

MONO2470

MONO2 2480

MONO2490

MONO2500

MONO2510

MONO2 520

MONO2530

MONO2540

MONO2550

MONO2560

MONO2 570

MONO2580

MONO2590

MONO2600

MONO2610

MONO2620

MONO2630

MONO2640

MONO2 650

MONO2660

MONO2670

MONO2 680

MONO2 690

MONO2700

MONO2710

MONO2720

MONO2730

MONO2740

MONO2750

MONO2760

MONO2770

MONO2 780

MONO2790

MONO2800

MONO2810

MONO2820

:


WRITE $(7,92)$ XRNET, XRADC, TIM, XNCTC, XN2

MONO2830

WRITE $(7, *) 11$

300

CONTINUE

STOP

MONO2840

MONO2850

MONO2860

END

MON02870 

